\title{
Mundos Brana: Buracos Negros e Buracos de Minhoca
}

\author{
Juliano Cesar Silva Neves
}

Orientador: Prof. Dr. Carlos Molina Mendes

Tese apresentada ao Instituto de Física da Universidade de São Paulo para a obtenção do título de Doutor em Ciências.

\section{Comissão Examinadora:}

Prof. Dr. Carlos Molina Mendes (EACH-USP)

Prof. Dr. Diego Trancanelli (IF-USP)

Prof. Dr. Marcos Vinicius Borges Teixeira Lima (IF-USP)

Prof. Dr. Jorge Ernesto Horvath (IAG-USP)

Prof. Dr. Olexandr Zhydenko (UFABC) 


\title{
FICHA CATALOGRÁFICA
}

\section{Preparada pelo Serviço de Biblioteca e Informação} do Instituto de Física da Universidade de São Paulo

\author{
Neves, Juliano Cesar Silva
}

Mundos brana: buracos negros e buracos de minhoca. São Paulo, 2012.

Tese (Doutorado) - Universidade de São Paulo. Instituto de Física - Depto. de Física Matemática

Orientador: Prof. Dr. Carlos Molina Mendes

Área de Concentração: Física

Unitermos: 1. Mundos brana; 2. Buracos negros;

3.Buracos de minhoca; 4. Ótica geométrica.

USP/IF/SBI-091/2012 


\section{Resumo}

Neste trabalho, construímos novas soluções com simetria esférica ou axial para as equações do campo gravitacional induzido num cenário brana do tipo Randall-Sundrum, onde uma 3-brana com constante cosmológica está imersa num espaço-tempo 5-dimensional conhecido como bulk. Para o caso esfericamente simétrico, com constante cosmológica negativa, obtivemos uma família de soluções numa brana assintoticamente anti-de Sitter, sendo que cada membro desta é diferenciado de outro por uma constante integração $C$, que fixada proveu-nos soluções de buracos negros ou buracos de minhocas. Com a mesma simetria, geometrias que descrevem buracos de minhoca foram encontradas numa brana assintoticamente de Sitter. Para o caso axialmente simétrico, métricas assintoticamente anti-de Sitter e de Sitter foram construídas no contexto citado. Propriedades óticas, como a rotação do vetor de polarização, foram estudadas, mostrando-nos algumas diferenças entre os mundos brana, tratados como uma teoria de gravitação modificada, e a relatividade geral. 



\section{Abstract}

In this work we have constructed new solutions, with axial or spherical symmetry, to the induced gravitational field equations within a Randall-Sundrum type brane world scenario, where a 3-brane with cosmological constant is embedded in a 5-dimensional space-time called bulk. For the spherical case with negative cosmological constant, we have found a family of solutions in an anti-de Sitter brane, where each member of the family is different from another when $C$ is fixed. With a fixed $C$ we have black holes or wormholes. In this same case, with spherical symmetry, we have obtained wormhole solutions in a de Sitter brane. In the same context, for the axially symmetric case, metrics asymptotically anti-de Sitter and de Sitter were built. Optics features, like rotation of polarization vector, have been studied. These features showed some differences between brane worlds, a theory of modified gravity, and the General Relativity. 


\section{Agradecimentos}

Ao orientador e à agência de fomento, os agradecimentos são imprescindíveis e quase obrigatórios. A Carlos Molina, agradeço pela leitura crítica desta tese e pelos frutos colhidos nos últimos anos — sem tua ajuda seriam impossíveis. À CAPES, agradeço pelo apoio financeiro nos últimos anos.

À minha família: pai, mãe, irmã mais velha e irmã mais nova — todo o caminho que tenho percorrido cruza os vossos caminhos. Agradeço-te, família.

Aos amigos, dedico as palavras de Zaratustra. Depois de dez anos, alimentando-se so-

mente do seu espírito e solidão, o sábio descobriu o que a amizade é. Àqueles que sabem ser e ter amigos, as palavras a seguir em vós foram inspiradas:

“És puro ar e solidão e pão e remédio para os teus amigos? Há quem não pode se soltar dos próprios grilhões e, no entanto, é um salvador para o amigo.

És um escravo? Então não podes ser amigo. És um tirano? Então não podes ter amigos." (Friedrich Nietzsche, Assim falou Zaratustra I, Do amigo) 
À vida que ascende. 

“(...) quanto mais afetos permitirmos falar sobre uma coisa, quanto mais olhos, diferentes olhos, soubermos utilizar para essa coisa, tanto mais completo será o nosso 'conceito' dela, nossa 'objetividade"”.

Friedrich Nietzsche, Genealogia da moral, III, 12. 



\section{Sumário}

1 Introdução 1

2 Mundos brana $\quad 7$

2.1 Dimensões extras . . . . . . . . . . . . . . . . . . . 7

2.2 Modelos Randall-Sundrum . . . . . . . . . . . . . . . . . . . . . . . . . . 10

2.3 Soluções a partir do bulk . . . . . . . . . . . . . . . . . . . . . . . . . . 14

2.4 Soluções a partir da brana . . . . . . . . . . . . . . . . . . . . . 18

3 Simetria esférica em mundos brana $\quad 23$

3.1 Soluções assintoticamente planas . . . . . . . . . . . . . . . . . . 23

3.2 Soluções assintoticamente AdS . . . . . . . . . . . . . . . . . 26

3.3 Soluções assintoticamente dS . . . . . . . . . . . . . . . . . . . . 37

3.4 Geodésicas . . . . . . . . . . . . . . . . . . . 42

3.4.1 Trajetórias radiais . . . . . . . . . . . . . . . . . . . . . 44

3.4 .2 Trajetórias não radiais $\ldots \ldots \ldots \ldots$. . . . . . . . . . 47

4 Simetria axial em mundos brana 49

4.1 Soluções de Kerr e Kerr-(anti)-de Sitter ～. . . . . . . . . . . . . . . . . . . 49

4.2 Solução assintoticamente plana . . . . . . . . . . . . . . . . . . . 53

4.3 Solução assintoticamente $\operatorname{AdS} \ldots \ldots \ldots$. . . . . . . . . . . 56

4.4 Solução assintoticamente $\mathrm{dS} \ldots \ldots \ldots 1$

4.5 Cargas conservadas . . . . . . . . . . . . . . . . . . . 68

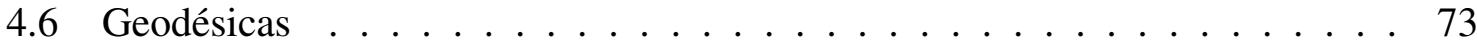

5 Ótica geométrica em espaços-tempo curvos

5.1 A abordagem de Pineault e Roeder . . . . . . . . . . . . . . . . . . . . . . 79

5.2 Aplicações às geometrias com simetria axial . . . . . . . . . . . . . . . 84

5.2 .1 Variação do vetor de polarização . . . . . . . . . . . . . . 86

5.2.2 Variação do cisalhamento na direção da congruência nula . . . . . . . 92 
6 Comentários finais $\quad 95$

A Equações do campo gravitacional numa 3-brana 97

B Métricas com simetria axial ou esférica $\quad 105$

$\begin{array}{lr}\text { C Horizontes } & 109\end{array}$

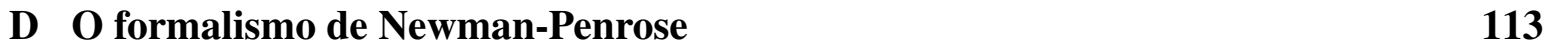

E Os referenciais localmente não girantes $\quad 121$ 


\section{Capítulo 1}

\section{Introdução}

Buracos negros e buracos de minhoca estão entre os frutos mais curiosos da física teórica. O primeiro, uma região do espaço-tempo sem conexão causal com aquilo que lhe é externo; o segundo, uma ponte entre distantes regiões do cosmo. Ambos, soluções das equações da teoria geométrica da gravitação de Albert Einstein: a teoria da relatividade geral.

Pouco tempo depois de sua formulação, em 1916, a teoria da relatividade geral foi apreciada e soluções — também conhecidas como métricas, geometrias, espaços-tempo — das chamadas equações de Einstein foram criadas. Uma das primeiras soluções foi proposta por Schwarzschild no mesmo ano de 1916 [1], uma geometria com simetria esférica; logo em seguida, uma geometria com a mesma simetria e um ingrediente a mais - a carga elétrica foi obtida, de forma independente, por Reissner [2] e Nordström [3]. Soluções com simetria axial, que descrevem corpos em rotação, foram somente obtidas décadas depois; como exemplo podemos citar a solução de Kerr em 1963 no Gravitational field of a spinning mass as an example of algebraically special metrics [4], talvez a mais importante nesta categoria. Dois anos mais tarde, no trabalho [5], Newman et al. colocaram carga elétrica na métrica de Kerr. A geometria de Kerr é assintoticamente plana, surge a partir das equações de Einstein com constante cosmológica nula; sua generalização para os casos em que a constante cosmológica é positiva, assintoticamente de Sitter (dS), ou a constante cosmológica é negativa, assintoticamente anti-de Sitter (AdS), foi realizada por Carter [6] em 1968. Ambos os trabalhos de Kerr e de Carter - referem-se a soluções quadridimensionais; suas generalizações para um número qualquer de dimensões foram realizadas quase duas décadas depois por Mayers e Perry, em 1986, no trabalho [7], onde generalizaram a solução de Kerr, e por Gibbons, Page e Pope [8] em 2005, onde generalizaram as soluções Kerr-dS e Kerr-AdS. Antes, vale a pena citar, o artigo de Hawking, Hunter e Taylor-Robinson [9] trouxe a solução em 5 dimensões para a métrica Kerr-AdS pela primeira vez na literatura científica. Todas as geometrias, solu- 
ções ou métricas citadas referem-se àquilo que hoje chamamos de buracos negros ${ }^{1}$. Tal termo somente foi cunhado em 1968, por John Wheeler, num artigo intitulado The Know and the Unknow, publicado na American Scientist e na American Scholar.

Já os buracos de minhoca aparecem na década de sessenta do século passado em estudos feitos também por John Wheeler. Entretanto, em tais estudos, as soluções encontradas não tinham uma característica importante, que somente nos trabalhos de Michael Morris e Kip Thorne [10] apareceu: a transitabilidade. Ou seja, em Morris e Thorne, buracos de minhoca são transitáveis, são passíveis de representar o papel de uma ponte ou túnel entre regiões distantes do universo. Então, um viajante, nesse caso, pode entrar e sair livremente de tal túnel, pode retornar e contar sua história. Além disso, em Morris e Thorne, buracos de minhoca apresentam outra característica perturbadora — surgem como possíveis máquinas do tempo. Mas só recentemente os buracos de minhoca têm ganhado atenção. Desde a detecção da expansão acelerada do universo [11, 12] em 1998 - promovida por um campo, a energia escura, que viola algumas condições de energia na relatividade geral —, buracos de minhoca tornaram-se mais interessantes. É justamente a existência de tal campo exótico que pode promover a realidade física de um buraco de minhoca.

Portanto, buracos negros e de minhoca são objetos físicos que devem ter uma realidade tal como estrelas e galáxias. Fortes indícios existem hoje sobre a existência de buracos negros, por exemplo, no centro de galáxias como a Via Láctea [13]. Por outro lado, mesmo com ainda nenhum indício, a existência de buracos de minhocas, como já dissemos, é hoje mais aceitável do que antes. De qualquer forma, ambos apresentam-se como objetos extremos na relatividade geral. Realidades físicas somente possíveis em condições extremas. São os limites da relatividade geral que surgem com tais objetos. No caso de buracos negros, a existência de singularidades em espaços-tempos einsteinianos, um limite à relatividade geral, é razoável, segundo os teoremas de Hawking e Penrose ${ }^{2}$. Mas em mundos brana, isso nem sempre é uma regra ${ }^{3}$.

Mundos brana têm atraído a atenção dos físicos desde a segunda metade dos anos noventa do século passado. Inspirado em teorias de cordas, o conceito de brana varia de acordo com o modelo. Em [15] — solução de Horava-Witten —, os campos de calibre estão confinados em duas 10-dimensionais branas; as 6 dimensões extras restantes são compactas com dimensões

\footnotetext{
${ }^{1}$ Para uma definição de buracos negros, veja o nosso apêndice $C$, onde a noção de horizonte de eventos foi apresentada. Tal noção é imprescindível para a definição desses objetos.

${ }^{2}$ Cf. [14], especialmente o capítulo 8.

${ }^{3}$ No capítulo 3 , mostramos uma geometria esfericamente simétrica com um horizonte de eventos e sem uma singularidade. São os chamados buracos negros regulares que possuem essa característica.
} 
muito reduzidas, da ordem da escala de Planck. Uma proposta 5-dimensional dessa solução foi feita em 1999 [16], onde a dimensão extra pode ser maior do que a escala citada anteriormente. Esta última tentativa deu origem à proposta do modelo Randall-Sundrum II (RS-II) [17], segundo o qual uma brana 4-dimensional está imersa num espaço-tempo 5-dimensional assintoticamente anti-de Sitter conhecido como bulk, sendo que todos os campos, exceto o gravitacional, são confinados na hipersuperfície quadridimensional; nesse caso, o raio da dimensão extra pode ser infinito. Antes, porém, estes mesmos autores propuseram o modelo Randall-Sundrum I (RS-I) [18], que contém duas branas 4-dimensionais imersas num espaçotempo 5-dimensional; nesse caso, a dimensão extra é compacta. Outro modelo inspirador é o modelo ADD [19], de Arkani, Dimopoulos e Dvali, com mais de uma dimensão extra, cujos tamanhos são da ordem do milímetro. As propostas citadas anteriormente ${ }^{4}$ surgem a partir da tentativa de resolver o problema da hierarquia, que é a enorme diferença entre a escala eletrofraca, $M_{f} \sim 10^{3} \mathrm{GeV}$, e a escala de massa de Planck 4-dimensional, $M_{p} \sim 10^{19} \mathrm{GeV}$. No caso do modelo RS-I, o problema da hierarquia é resolvido com o uso de um fator (warp factor) que se apresenta na métrica do bulk, tornando-a não fatorável, capaz de explicar a enorme diferença entre as escalas citadas.

Com uma nova visão, o que nós chamamos de mundo, para os modelos brana, é apenas uma parte dele. Nos mundos brana nosso universo visível, quadridimensional, está imerso num espaço-tempo 5-dimensional. Com essa nova visão, uma nova física pôde surgir. Em física de partículas, os mundos brana trouxeram novas partículas e modelos além do chamado modelo padrão das interações fundamentais [22]; na cosmologia, trouxeram o modelo DGP [23], de Dvali, Gabadadze e Porrati, além das cosmologias com ricochete (bouncing cosmologies $^{5}$ ), que tornam o big bang (a grande explosão), há quase quatorze bilhões de anos atrás, dispensável. Mas esta tese tem o intuito de explorar a gravitação trazida à luz pelos mundos brana. Nos capítulos 3 e 4 expusemos geometrias que nascem nesse frutífero contexto.

Com os mundos brana construídos, a gravitação nesse contexto foi estudada. Já em 2000, pouco tempo depois do surgimento dos modelos Randall-Sundrum, Chamblin, Hawking e Reall, no Brane-world black holes [25], construíram a chamada corda negra. Em tal solução, a métrica na brana é Schwarzschild, e a geometria no bulk mostrou-se instável diante de perturbações. Por outro lado, uma nova abordagem surgiu a partir do seminal trabalho de Shiromizu, Maeda e Sasaki, The Einstein equations on the 3-brane world [26]; nesse importante trabalho, a partir de um bulk 5-dimensional, as equações do campo gravitacional foram projetadas numa 3-brana ou brana quadridimensional. Com esse importante trabalho, estudos

\footnotetext{
${ }^{4}$ Uma revisão sobre o tema "mundos brana" pode ser obtida em [20, 21].

${ }^{5}$ É digno de nota enfatizar que ricochetes não surgem apenas em mundos brana. Seja em modelos $f(R)$, ou em gravidade quântica em loop, ou em mundos branas, ricochetes surgem. Cf. [24], especialmente a seção 6 que trata de ricochetes em cosmologias de branas.
} 
de buracos negros, buracos de minhoca e até de cosmologia foram realizados. A partir dessas equações projetadas, que no limite de baixas energias tornam-se as equações de Einstein, Casadio et al. [27] e Bronnikov et al. [28] construíram métricas de buracos negros e buracos de minhoca num contexto do tipo RS. Na vereda aberta por esses últimos autores citados, nós realizamos o trabalho exposto nesta tese.

Com as equações do campo gravitacional deduzidas por Shiromizu et al., exploramos em Black holes and wormholes in AdS branes [29] geometrias com simetria esférica numa brana com constante cosmológica negativa. Nossos resultados generalizaram os trabalhos [27, 28], pois nestes a constante cosmológica na brana é nula. Em nosso trabalho, uma família de soluções foi obtida: cada membro de tal difere de outro ajustando-se uma constante de integração $C$. Em tal família, identificamos buracos negros regulares e singulares, além de buracos de minhoca, como pode ser visto na seção 3.2.

Já em Wormholes in de Sitter branes [30], mostramos um equívoco no já citado trabalho de Bronnikov et al. Em tal trabalho, os autores afirmam que a única métrica numa brana com constante cosmológica positiva é a solução de Sitter. Mas em nosso artigo, construímos uma família de buracos de minhoca nesse mesmo contexto, discordando, assim, de Bronnikov et al. Uma característica desses buracos de minhoca - além do comportamento assintótico é serem transitáveis. Ou seja, esses não possuem um horizonte de eventos que os protegem. Sendo assim, como já afirmamos anteriormente, podem servir como túnel entre distantes regiões do espaço-tempo em que são gerados.

Em todas as referências citadas até o momento, temos somente soluções com simetria esférica em cenários do tipo Randall-Sundrum. Mas em 2002, Modgil et al. [31] anunciaram uma solução com simetria axial, ou um buraco negro em rotação, no contexto de mundos brana, onde a generalização da métrica com simetria esférica numa 3-brana Ricci-plana, obtida por Chamblin et al. [25], foi mostrada. Seguindo o trabalho de Dadhich et al. [32], que usaram simetria esférica, Aliev e Gümrükçüoglu no Charged rotating black holes on a 3-brane [33] construíram métricas axialmente simétricas, sem constante cosmológica, com ou sem campo de Maxwell na brana. Em ambos casos — Dadhich et al. e Aliev et al. — foi notado o aparecimento na brana de "cargas de maré", cargas do tipo Coulomb; tais medem a influência do bulk na brana. Além da simetria adotada, a diferença entre os trabalhos de Dadhich et al. e Aliev et al. está nas equações projetadas na brana - as equações do campo gravitacional - , com o primeiro tendo usado o trabalho de Shiromizu et al. [26] e o segundo tendo se apoiado num trabalho de própria autoria [34].

Em Rotating black holes in a Randall-Sundrum brane with a cosmological constant [35], propusemos generalizar também os resultados de Aliev e Gümrükçüoglu [33], adotando na brana constante cosmológica negativa ou positiva. No capítulo 4 ambas estão expostas. Em 
ambos os casos, construímo-las a partir do chamado ansatz de Kerr-Schild-(A)-dS. Como resultado, a estrutura das geometrias - no caso assintoticamente AdS e assintoticamente dS — assemelham-se às métricas Kerr-Newman-(A)dS no contexto einsteiniano. Mesmo tendo usado um ansatz sem carga, tal como Aliev e Gümrükçüoglu, a "carga de maré" $q$ apresentouse como uma carga coulombiana, quando a métrica foi escrita nas coordenadas de BoyerLindquist. Também em nossos resultados, o uso de valores negativos para $q$ amplificam os efeitos gravitacionais do bulk na brana. Ou seja, o raio do horizonte de eventos, a ergosfera, por exemplo, assumiram valores maiores quando $q<0$. Como pôde-se notar pelas "cargas de maré", buracos negros em mundos brana oferecem resultados que não têm um correspondente na relatividade geral. Com as métricas nas mãos, obtivemos as equações das geodésicas, que foram indispensáveis para a aplicação que delas fizemos: um estudo de ótica geométrica em geometrias com simetria axial.

Desde a metade do século passado, relativistas acreditam que um campo gravitacional, gerado por um corpo em rotação, altera o plano de polarização de ondas eletromagnéticas que o circundam. Devido a esse fenômeno, segundo Brodutch et al. [36], haveria a alteração da polarização de raios X provenientes de discos de acreção no entorno de buracos negros. Como Pineault e Roeder [37], Frederick et al. [38] estimaram a variação do vetor de polarização na geometria de Kerr. Os últimos utilizaram não somente os referenciais localmente não girantes - referencias que rodam junto com o buraco negro, de acordo com o apêndice E -, mas também usaram o chamado referencial global inercial, onde a direção da base de vetores permanece inalterada em relação às estrelas fixas. Utilizamos, nesta tese, a abordagem de Pineault e Roeder, descrita em Applications of geometrical optics to the Kerr metric, na qual o chamado formalismo de Newman-Penrose foi usado para a estimativa de grandezas óticas de interesse. Tal formalismo - uma opção desenvolvida na segunda metade do século XX para apresentar a relatividade geral - surgiu no trabalho An approach to gravitational radiation by a method of spin coefficients [39], de 1962, desenvolvido para o estudo da radiação gravitacional. Os autores, a partir da escolha de uma tetrada nula (uma base de quatro vetores do tipo luz ou tipo nulo ${ }^{6}$ ), obtiveram 12 constantes, chamadas coeficientes de spin - importantes para avaliar quantidades óticas de um espaço-tempo, como pode ser visto no apêndice D. Por outro lado, a utilização da tetrada nula também pode ser indicada para radiação eletromagnética, ou seja, para luz — como foi feito por Pineault e Roeder [37] para métrica de Kerr, como já dissemos —, pois o vetor tangente de uma congruência nula (um feixe de luz) é igualmente do tipo luz.

\footnotetext{
${ }^{6} \mathrm{O}$ uso das tetradas nulas pôde tornar as equações envolvidas mais simples — todas de primeira ordem sem envolver uma base de coordenadas quando um tensor foi escrito.
} 
No capítulo 5, utilizamos o formalismo de Newman-Penrose, tal como Pineault e Roeder, e calculamos a variação do vetor de polarização e a variação do cisalhamento da luz em trajetórias nas geometrias axiais, no contexto de branas que escolhemos, estudadas no capítulo 4. A existência do bulk alterou as duas grandezas óticas mencionadas em relação à relatividade geral. Aqui, novamente, temos uma amplificação dos efeitos do bulk na brana com a escolha da "carga de maré" $q$ negativa. Podemos, então, considerá-los um possível teste que nos mostraria os vestígios da dimensão extra.

Todos os resultados mostrados nesta tese estão expressos nas unidades geométricas segundo as quais a velocidade da luz e a constante gravitacional 4-dimensional valem 1 -, e as métricas têm assinatura $(-,+,+,+)$. 


\section{Capítulo 2}

\section{Mundos brana}

Neste capítulo, discutimos a origem da necessidade por dimensões extras e os chamados mundos brana, modelos que adotam dimensões além das quatro habituais. Demos ênfase aos modelos Randall-Sundrum, que surgiram no final do século passado. Além disso, elencamos dois possíveis caminhos para obter-se soluções de buracos negros e buracos de minhoca nesse contexto: construção de métricas a partir da brana e a partir do espaço-tempo 5-dimensional — no qual o nosso mundo 4-dimensional está imerso —, conhecido como bulk.

\subsection{Dimensões extras}

A utilização de dimensões extras para descrever fenômenos físicos data dos pioneiros trabalhos de Kaluza e Klein, em anos da década de 1920. Atualmente, dimensões extras são um ingrediente indispensável na mais popular candidata à teoria unificada na física: a teoria de cordas. Caso sejam consideradas fisicamente reais, algumas questões se colocam: (i) como essas dimensões extras podem alterar ou afetar o mundo sensível, que é, desde a relatividade de Einstein, um continuum quadridimensional?; (ii) por que não as observamos diretamente?

Segundo Newton, em sua lei da gravitação universal, o módulo da força entre duas partículas de massas $m_{1}$ e $m_{2}$, separadas pela distância $r$, é dado por $G_{N} m_{1} m_{2} / r^{2}$, onde $G_{N}$ é a constante gravitacional newtoniana em 4 dimensões. $O$ fato da força depender somente da distância relativa entre os corpos pode ser creditado como uma consequência da isotropia do espaço, ou seja, as leis físicas são independentes da direção, são as mesmas em qualquer direção. A constante gravitacional newtoniana conduz a uma escala fundamental, a escala de Planck ou massa de Planck 4-dimensional $\left(M_{p}\right)$. Quando se adota $h / 2 \pi=c=1$ (onde $h$ é a constante de Planck, e $c$ é a velocidade da luz), em unidades naturais, $G_{N}\left(=10^{-66} \mathrm{~cm}^{2}\right)$ 
conduz ao seguinte valor para aquela escala:

$$
G_{N}=\frac{1}{M_{P}^{2}} \Rightarrow M_{p}=10^{19} \mathrm{Gev} .
$$

Com a suposição de dimensões extras, a ação gravitacional de Einstein-Hilbert torna-se

$$
S=\frac{1}{16 \pi G_{4+d}} \int d^{4} x d^{d} y \sqrt{-{ }^{(4+d)} g}\left[{ }^{(4+d)} R-2 \Lambda_{4+d}\right],
$$

sendo $d$ o número de dimensões extras, $x$, coordenadas nas dimensões usuais, $y$, coordenadas nas dimensões extras, $G_{4+d},{ }^{(4+d)} g,{ }^{(4+d)} R$ e $\Lambda_{4+d}$ são a constante gravitacional, a métrica, o escalar de curvatura ou escalar de Ricci e a constante cosmológica em $4+d$ dimensões, respectivamente. Segundo Maartens ${ }^{1}$, numa interessante revisão sobre esse tema, a ação (2.2) conduz à seguinte equação para o campo gravitacional:

$$
{ }^{(4+d)} G_{A B} \equiv{ }^{(4+d)} R_{A B}-\frac{1}{2}{ }^{(4+d)} R^{(4+d)} g_{A B}=-\Lambda_{4+d}{ }^{(4+d)} g_{A B}+8 \pi G_{4+d}{ }^{(4+d)} T_{A B},
$$

$\operatorname{com} X^{A}=\left(x^{0}, \ldots, x^{3}, y^{0}, \ldots, y^{d}\right)$. A relação entre a constante gravitacional em $4+d$ dimensões e a massa de Planck em $4+d$ dimensões é semelhante ao caso quadridimensional

$$
G_{4+d}=\frac{1}{M_{4+d}^{2+d}}
$$

No limite do campo fraco, onde vale a gravitação de Newton, as equações (2.3) conduzem a uma equação de Poisson $(4+d)$-dimensional , cuja solução é o potencial gravitacional

$$
V(r) \propto \frac{8 \pi G_{4+d}}{r^{1+d}} .
$$

Considerando que a escala ou a ordem do comprimento das dimensões extras seja $L$, em escalas menores do que esta $(r \lesssim L)$, o potencial gravitacional será $(4+d)$-dimensional $\left(V(r) \sim r^{-(1+d)}\right)$. Por outro lado, quando a escala em foco for da ordem de $L$ ou maior, o potencial gravitacional apresentar-se-á como um potencial 4-dimensional, ou seja, $V(r) \sim$ $L^{-d} r^{-1}$, tornando irrelevante a influência das dimensões extras. Então, para objetos macroscópicos, o módulo da força gravitacional será proporcional à razão $1 / r^{2}$, como tem sido medido. Quando são feitos experimentos a curtas distâncias, outras forças, como a força elétrica, interferem na medida da força gravitacional - pois a gravitação é fraca a curtas distâncias. Os melhores dados obtidos por [40] mostram que abaixo de $0,1 \mathrm{~mm}$ não pode-

\footnotetext{
${ }^{1}$ Cf. [20], cap. 1.
} 
mos discernir entre a força gravitacional e as demais forças. Portanto, a lei da força, que diminui com $1 / r^{2}$, funciona muito bem apenas entre a escala astronômica - distâncias da ordem do sistema solar - e $0,1 \mathrm{~mm}$. Se dimensões extras existirem, sua importância será notada somente em medidas abaixo de $0,1 \mathrm{~mm}$. De uma forma geral, num mundo $(4+d)$ dimensional, a lei da gravitação diminuirá com $1 / r^{2+d}$ e será inversamente proporcional à massa de Planck $(4+d)$-dimensional, conforme (2.4) e a definição da força. Dessa forma, as massas ou escalas de Planck 4-dimensional e $(4+d)$-dimensional relacionar-se-ão por

$$
M_{p}^{2} \sim M_{4+d}^{2+d} L^{d}
$$

onde $L^{d}$ é o volume das $d$ dimensões adicionais. Neste contexto, a constante $M_{p}$ será uma constante de acoplamento efetiva, relacionada à escala fundamental, $M_{4+d}$. Quando $L^{d}$ for da ordem de $M_{p}$, teremos $M_{4+d} \sim M_{p}$. Quando isso não ocorre, especialmente no caso $L^{d} \gtrsim M_{p}$, a escala fundamental poderá ser muito menor do que a escala em 4 dimensões: $M_{4+d} \ll M_{p} \sim$ $10^{19} \mathrm{Gev}$. Com $M_{4+d}$ pequeno, a fraqueza da gravidade a pequenas distâncias explicar-se-á pelo seu transbordamento para as $d$ dimensões extras.

Em 1921, Kaluza, na tentativa de unificar o eletromagnetismo com a gravitação, propôs um espaço-tempo com 4 dimensões espaciais e uma temporal. As dimensões espaciais eram: 3 dimensões infinitas e uma compacta de raio $r_{c}$. Poucos anos depois, Klein, em 1926, sugeriu que o tamanho de tal dimensão compacta seria da ordem da escala de Planck, $10^{-33} \mathrm{~cm}-$ algo totalmente inobservável. Então, a gravitação seria consistente com o que observamos - mesmo com a isotropia do espaço violada, pois as demais dimensões são distinguidas da dimensão extra devido às linhas de força só poderem propagar-se até uma distância finita na dimensão compacta —, em largas escalas ou distâncias $\left(r>r_{c}\right)$, teríamos a adequação da lei da gravitação a um espaço-tempo quadridimensional (3 dimensões espaciais e 1 dimensão temporal) e a sua diminuição com $1 / r^{2}$. Em pequenas distâncias $\left(r<r_{c}\right)$, a lei da força diminuiria com $1 / r^{3}$.

Como vimos, nos trabalhos de Kaluza e Klein as dimensões extras tinham tamanho muito reduzido — da ordem da escala de Planck. Mesmo para teoria de cordas, as 6 dimensões extras que necessita têm essa mesma ordem de grandeza ${ }^{2}$. Entretanto, é possível pensar numa dimensão extra não tão reduzida, mesmo até com um raio infinito. Em 1996, o trabalho [45] indicou que tais dimensões extras podem ser menores que $10^{3} \mathrm{GeV}$, isto é, 16 ordens de grandeza maiores do que a escala de Planck quadridimensional $\left(10^{19} \mathrm{GeV}\right)$. Além disso, através do trabalho de Arkani-Hamed, Dimopoulos e Dvali [19], que deu origem ao modelo ADD, a previsão para o raio da dimensão compacta no caso de 2 dimensões extras pode ser $1 \mathrm{~mm}-$

\footnotetext{
${ }^{2}$ Cf. [44], que faz uma revisão sobre o tema das cordas.
} 
um valor razoável e passível de ser testado. O modelo ADD utiliza um conceito proveniente da teoria de cordas que será modificado e utilizado nos modelos Randall-Sundrum, o conceito de brana. O termo brana deriva-se de membrana e significa, sem muito rigor, uma superfície imersa num espaço com maior número de dimensões. Para a teoria de cordas, existem subvariedades $(p+1)$-dimensionais imersas num bulk 10-dimensional chamadas $p$-branas. Entre as $p$-branas existem as $D$-branas, que são extremidades de cordas abertas (1-brana). Assim, no modelo ADD temos um espaço tempo $(4+d)$-dimensional, com as $d$ dimensões extras compactas, contornado por branas onde todos os campos, exceto o gravitacional, estão confinados nas subvariedades. A motivação do modelo ADD foi resolver o problema da hierarquia, que é a diferença entre a escala de massa eletrofraca, $M_{f} \sim 10^{3} \mathrm{GeV}$, e a escala de massa de Planck quadridimensional, $M_{p} \sim 10^{19} \mathrm{GeV}$.

Mas qual é a origem da diferença entre as escalas $M_{f}$ e $M_{p}$ ? A diferença entre escala eletrofraca, dada pelo valor esperado no vácuo do bóson de Higgs, e a escala de Planck, $M_{p}$, já foi buscada em algumas propostas ${ }^{3}$, tais como a supersimetria e o modelo technicolor. Modelos com dimensões extras também estão entre as tentativas para a resolução desse problema. No modelo ADD, a única escala natural é a escala eletrofraca, sendo a planckiana em $(4+d)$-dimensões da mesma ordem daquela. Da mesma forma, no modelo Randall-Sundrum I (RS-I), a escala planckiana em cinco dimensões é tida da ordem da escala eletrofraca. Para resolver o problema da hierarquia, no RS-I, com uma dimensão extra, um fator exponencial foi adicionado à métrica do espaço-tempo 5-dimensional — no qual nosso mundo quadridimensional está imerso.

Entretanto, a dimensão extra pode ser maior do que alguns milímetros, pode ser infinita, como veremos no modelo Randall-Sundrum 2 (RS-II), que surge a partir do modelo RandallSundrum I.

\subsection{Modelos Randall-Sundrum}

Existem dois modelos Randall-Sundrum, ambos utilizam a ideia de um bulk de 5 dimensões, sendo que todos os campos, exceto o gravitacional, estão confinados numa brana que pode representar o nosso universo quadridimensional. O modelo Randall-Sundrum I (RS-I) consiste de duas branas quadridimensionais imersas num bulk, com o tamanho da dimensão extra finito. Por outro lado, o modelo Randall-Sundrum II (RS-II) consiste de uma brana quadridimensional também imersa num bulk, mas o tamanho da dimensão extra pode ser infinito. Os modelos Randall-Sundrum, como já dissemos, foram desenvolvidos, motivados, pelo problema da hierarquia. Nesses, a gravidade é mais forte perto da brana e diminui expo-

\footnotetext{
${ }^{3}$ Cf. [22], uma sucinta descrição sobre algumas tentativas de eliminar o problema da hierarquia.
} 
nencialmente quando nos afastamos dela, na dimensão extra. Justamente por isso, a métrica no bulk tem um fator exponencial que depende da nova dimensão, é função da coordenada extra. E é esse novo fator (warp factor) na métrica que atenua a gravidade e corrige o problema da hierarquia no RS-I.

De acordo com o artigo Large mass hierarchy from a small extra dimension [18], o modelo RS-I adota duas 3-branas imersas num bulk 5-dimensional anti-de Sitter (AdS) - com simetria $S^{1} / \mathbb{Z}_{2}$ - cuja coordenada extra $(y)$ é compacta com raio $r_{c}$. Uma das branas pode ser identificada com o nosso universo visível, a outra, com um universo escondido; ambas possuem posições fixas no bulk. Assim, podemos restringir o intervalo de interesse da coordenada $y$ colocando uma brana em $y=0$, a brana visível, e a outra em $y=\pi r_{c}$, a brana escondida. Na figura 2.1, é representada uma imagem deste modelo com uma dimensão espacial suprimida.

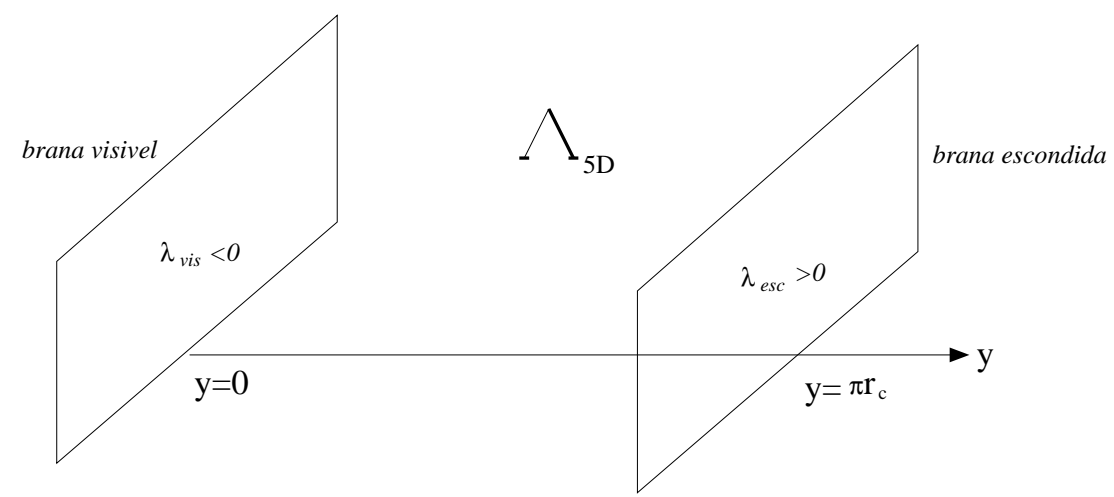

Figura 2.1: Representação do modelo RS-I onde uma dimensão espacial foi suprimida. As duas branas, visível e escondida, possuem tensão negativa e positiva, respectivamente. Ambas estão imersas num bulk antide Sitter com constante cosmológica $\Lambda_{5 D}$.

Neste modelo, as métricas das duas 3-branas são escritas como

$$
\begin{aligned}
& g_{\mu \nu}^{v i s}={ }^{(5)} g_{A B}(y=0) \delta_{\mu}^{A} \delta_{v}^{B}, \\
& g_{\mu \nu}^{e s c}={ }^{(5)} g_{A B}\left(y=\pi r_{c}\right) \delta_{\mu}^{A} \delta_{v}^{B},
\end{aligned}
$$

onde ${ }^{(5)} g_{A B}$ é a métrica no bulk (os índices gregos vão de 0 a 3, e os índices latinos vão de 0 a 4). Sua ação clássica é dada por

$$
S=S_{5 D}+S_{v i s}+S_{e s c}
$$

sendo $S_{5 D}, S_{v i s}, S_{e s c}$ as ações no bulk, brana visível e brana escondida, respectivamente. As 
expressões para cada componente da ação são

$$
\begin{gathered}
S_{5 D}=\int d^{4} x \int_{0}^{\pi r_{c}} d y \sqrt{-{ }^{(5)} g}\left[-\Lambda_{5 D}+2 M_{5 D}^{3}{ }^{(5)} R\right] \\
S_{v i s}=\int d^{4} x \sqrt{-g_{v i s}}\left[\mathscr{L}_{v i s}-\lambda_{\text {vis }}\right]
\end{gathered}
$$

e

$$
S_{e s c}=\int d^{4} x \sqrt{-g_{e s c}}\left[\mathscr{L}_{e s c}-\lambda_{e s c}\right]
$$

onde ${ }^{(5)} g, g_{\text {vis }}$ e $g_{\text {esc }}$ são os determinantes das métricas do bulk, brana visível e brana escondida, respectivamente. A constante cosmológica em 5 dimensões é representada por $\Lambda_{5 D}$, e $M_{5 D}$ é a massa de Planck 5-dimensional. ${ }^{(5)} R$ é o escalar de Ricci 5-dimensional, $\lambda_{v i s}$ é a tensão na brana visível, e $\lambda_{\text {esc }}$ é a tensão na brana escondida, ambas atenuam o efeito da constante cosmológica do bulk. De acordo com os autores, as lagrangianas $\mathscr{L}_{\text {vis }}$ e $\mathscr{L}_{\text {esc }}$ das 3-branas não precisam ser conhecidas em detalhes para se determinar a métrica do bulk. As integrais acima são em relação a $x^{\mu}$, coordenada na brana, e $y$, coordenada da dimensão extra.

Podemos obter as equações de Einstein em 5 dimensões extremizando a ação (2.9). Com isso feito, as equações de campo apresentam-se como

$$
\begin{gathered}
\sqrt{-{ }^{(5)} g}\left({ }^{(5)} R_{A B}-\frac{1}{2}{ }^{(5)} g_{A B}{ }^{(5)} R\right)=-\frac{1}{4 M_{5 D}^{3}}\left[\sqrt{-g_{v i s}} g_{\mu \nu}^{v i s} \delta_{A}^{\mu} \delta_{B}^{v} \lambda_{v i s} \delta(y)\right. \\
\left.+\sqrt{-{ }^{(5)} g^{(5)}} g_{A B} \Lambda_{5 D}+\sqrt{-g_{\text {esc }}} g_{\mu \nu}^{e s c} \delta_{A}^{\mu} \delta_{B}^{v} \lambda_{\text {esc }} \delta\left(y-\pi r_{c}\right)\right] .
\end{gathered}
$$

Assumindo que existe uma solução quadridimensional, uma solução na brana, que obedece a invariância de Poincaré nas direções indicadas a partir das coordenadas $x^{\mu}$, pode-se usar a métrica 5-dimensional

$$
d s^{2}=e^{2 \sigma(y)} \eta_{\mu v} d x^{\mu} d x^{v}+d y^{2}
$$

como ansatz em (2.13), onde a métrica quadridimensional $\eta_{\mu \nu}$ é a métrica de Minkowski. Dessa forma, as equações de Einstein reduzem-se ao par

$$
6 \sigma^{\prime 2}=-\frac{\Lambda_{5 D}}{4 M_{5 D}^{3}}
$$

$\mathrm{e}$

$$
3 \sigma^{\prime \prime}=\frac{\lambda_{v i s} \delta(y)}{2 M_{5 D}^{3}}+\frac{\lambda_{e s c} \delta\left(y-\pi r_{c}\right)}{2 M_{5 D}^{3}} .
$$

Os símbolos linha “/” indicam derivação com respeito à coordenada $y$. A solução para (2.15) 
que respeita a simetria $S^{1} / \mathbb{Z}_{2}$, ou seja, $y \rightarrow-y$, é

$$
\sigma=|y| \sqrt{-\frac{\Lambda_{5 D}}{24 M_{5 D}^{3}}} .
$$

Vemos, em (2.17), que somente a solução aceitável é aquela com $\Lambda_{5 D}$ negativa. Portanto, o espaço-tempo entre as duas branas é tido como $A d S_{5}$.

Como a métrica (2.14) é uma função periódica em $y$ (solução com simetria $S^{1} / \mathbb{Z}_{2}$ ), com a derivação de (2.17), válida no intervalo $0 \leq y \leq \pi r_{c}$, obtém-se

$$
\sigma^{\prime \prime}=2 r_{c} \sqrt{-\frac{\Lambda_{5 D}}{24 M_{5 D}^{3}}}\left\{\delta(y)-\delta\left(y-\pi r_{c}\right)\right\}
$$

Então, temos (2.16) - a partir de (2.18) - somente se $\lambda_{v i s}, \lambda_{e s c}$ e $\Lambda_{5 D}$ estiverem relacionadas com um escalar $k$ da seguinte maneira

$$
\lambda_{v i s}=-\lambda_{e s c}=24 M_{5 D}^{3} k \quad \text { e } \quad \Lambda_{5 D}=-24 M_{5 D}^{3} k^{2},
$$

sendo $k$ uma escala da ordem da escala planckiana. As relações acima entre os termos cosmológicos do bulk e da borda são fundamentais para obter-se uma solução que respeite a invariância de Poincaré quadridimensional na brana; mantendo-as, a métrica do bulk torna-se

$$
d s^{2}=e^{-2 k|y|} \eta_{\mu v} d x^{\mu} d x^{v}+d y^{2}
$$

para ambos os modelos, RS-I e RS-II, sendo que este último adota $r_{c} \rightarrow \infty$.

Fazendo $k=1 / l$ e introduzindo a coordenada $z=l e^{y / l} \mathrm{em}(2.20)$, temos a métrica

$$
d s^{2}=\frac{l^{2}}{z^{2}}\left(d z^{2}+\eta_{a b} d x^{a} d x^{b}\right),
$$

(onde $l$ é o raio AdS), que é conforme à métrica plana (com fator conforme $l^{2} / z^{2}$ ). A geometria de Minkowski em (2.21), que é o segundo termo entre parênteses, pode ser substituída por qualquer métrica Ricci plana e, ainda assim, será também solução de $(2.13)^{4}$.

Para, a partir da métrica (2.20), resolver o problema da hierarquia, Randall e Sundrum relacionaram a massa de Planck efetiva $\left(M_{P}\right)$, quadridimensional, com massa de Planck 5dimensional, $M_{5 D}$. Para isso, identificaram os modos zero da solução clássica — flutuações gravitacionais não massivas - substituindo a métrica de Minkowski $\eta_{\mu v}$, em (2.20), pela

\footnotetext{
${ }^{4}$ Como foi feito em [25], como veremos, na seção 2.3, substituindo $\eta_{\mu \nu}$ pela métrica de Schwarzschild.
} 
métrica

$$
\bar{g}_{\mu \nu}=\eta_{\mu v}+\bar{h}_{\mu \nu},
$$

que os conduziu a uma ação efetiva na brana visível. Com a métrica perturbada, com o auxílio da ação clássica (2.9), chegaram ao seguinte resultado:

$$
S_{e f} \approx \int d^{4} x \int_{0}^{\pi r_{c}} d y 2 M_{5 D}^{3} r_{c} e^{-2 k|y|} \sqrt{-\bar{g}} \bar{R},
$$

onde $\bar{R}$ é o escalar de Ricci proveniente da métrica perturbada (2.22). Para determinar $S_{e f}$, a ação quadridimensional efetiva, realizaram a integração na dimensão extra, identificando-a à massa de Planck efetiva. Ou seja,

$$
M_{p}^{2}=2 M_{5 D}^{3} r_{c} \int_{0}^{\pi r_{c}} d y e^{-2 k|y|}=\frac{M_{5 D}^{3}}{k}\left(1-e^{-2 k r_{c} \pi}\right)
$$

pôde conduzi-los à igualdade entre as escalas eletrofraca $\left(\sim 10^{3} \mathrm{Gev}\right)$ e a planckiana em cinco dimensões, desde que $k$ fosse da ordem de $10^{2} M_{5 D}^{-1}$ (ou $l=10^{-2} M_{5 D}$ ), e a distância entre as branas fosse tal que a razão $r_{c} / l$ valesse 35 . Com isso, a escala $M_{p}$ efetiva adquiriu seu valor da ordem de $10^{19} \mathrm{Gev}$, como se requer na gravitação quadridimensional.

Já no modelo Randall-Sundrum II, de acordo com An alternative to compactification [17], apenas uma 3-brana foi utilizada; como no RS-I, o bulk tem uma geometria anti-de Sitter, $A d S_{5}$; além disso, possui simetria $\mathbb{Z}_{2}$ e métrica expressa por (2.20). Nesse caso, a coordenada $y$ pode tender ao infinito, ou seja, ao contrário do modelo anterior, o RS-II não utiliza uma dimensão extra compacta $-r_{c}$ é infinito. No RS-II, o fator exponencial na métrica do bulk assegura que a gravidade se localize perto da brana. Fazendo $r_{c} \rightarrow \infty \mathrm{em}$ (2.24), não se resolve o problema da hierarquia, mas o interesse no RS-II dá-se pelo meio segundo o qual a relatividade geral é restaurada.

\subsection{Soluções a partir do bulk}

Nesta seção, a construção de soluções das equações do campo gravitacional conduz a uma completa solução. Ou seja, na abordagem aqui descrita, obtêm-se soluções na brana e no bulk, ao mesmo tempo. A primeira tentativa de construir uma solução de buracos negros num cenário como o RS-II foi feita por Hawking et al. em Brane-world black holes [25]. Nessa tentativa, os autores trocaram, na métrica para o bulk (2.20), a solução de Minkowski pela de Schwarzschild. Isto é, 


$$
d s^{2}=e^{-2 k|y|}\left[-\left(1-\frac{2 M}{r}\right) d t^{2}+\left(1-\frac{2 M}{r}\right)^{-1} d r^{2}+r^{2}\left(d \theta^{2}+\operatorname{sen}^{2} \theta d \phi^{2}\right)\right]+d y^{2} .
$$

Entre as interessantes características da métrica acima — que satisfaz as equações de campo do modelo Randall-Sundrum - estão a não necessidade de alguma matéria extra no bulk e o fato de que sua projeção na brana é uma solução muito bem conhecida: a solução de Schwarzschild. Entretanto, em 5 dimensões, a solução (2.25) conduz ao seguinte resultado para o escalar de Kretschmann:

$$
R_{A B C D} R^{A B C D} \sim \frac{48 M^{2} e^{4 k|y|}}{r^{6}} .
$$

Esse escalar diverge não somente na brana em $r=0$ e $y=0$, mas a sua divergência estende-se por toda a coordenada extra $y$. Temos, então, nesse resultado, a justificativa para interpretar (2.25) como uma corda negra, ou seja, como uma singularidade protegida por um horizonte de eventos, que se faz notar além da brana. Entretanto, a solução de Hawking et al. mostrouse instável a perturbações lineares, vitimada pelas já conhecidas instabilidades de GregoryLaflamme, de acordo com [41, 42].

Um outro caminho foi traçado por Creek, Gregory, Kanti e Mistry no trabalho Braneworld stars and black holes [43]. Nesse artigo, os autores assumiram uma métrica no bulk, $g_{A B}$, com simetria esférica escrita como:

$$
d s^{2}=-U(r) d t^{2}+\frac{1}{U(r)} d r^{2}+r^{2}\left(d \chi^{2}+\operatorname{sen}^{2} \chi d \Omega^{2}\right)
$$

onde $U(r)$ é uma função geral, e $d \Omega^{2}$ é a métrica de uma 2-esfera. Nessa abordagem, consideraram um espaço-tempo simétrico a reflexões no entorno de uma brana (simetria $\mathbb{Z}_{2}$ ) com tensão positiva, cuja função $\chi(t, r)$ indica a sua posição em relação à dimensão extra. Para construir a métrica induzida quadridimensional, definiram uma base em termos de vetores tangentes não normalizados e de um vetor unitário normal à brana

$$
T^{A}=(1,0, \dot{\chi}, 0,0), \quad R^{A}=\left(0,1, \chi^{\prime}, 0,0\right), \quad n_{A}=n\left(-\dot{\chi},-\chi^{\prime}, 1,0,0\right),
$$

com o ponto, acima do $\chi$, indicando derivada parcial em relação à coordenada $t$, o símbolo linha denotando derivação em relação à coordenada $r$ e $n^{2}=\left(U r^{2}\right) /\left(-r^{2} \dot{\chi}^{2}+U^{2} r^{2} \chi^{\prime}+U\right)$. Então, com o tensor

$$
h_{A B}=g_{A B}-n_{A} n_{B},
$$

projetaram vetores na brana, com suas componentes tangentes a definir uma métrica induzida. $\mathrm{O}$ vetor $n^{A}$ é ortogonal à brana e ao vetor $e_{\mu}^{A}$, tangente ao espaço-tempo quadridimensional. 
Com este último, puderam relacionar a métrica 5-dimensional com a métrica induzida, isto é,

$$
h_{\mu \nu}=e_{\mu}^{A} e_{v}^{B} g_{A B}=e_{\mu}^{A} e_{v}^{B} h_{A B}
$$

O principal ingrediente extra nessa abordagem são as condições de junção de Israel, que nesse caso, com uma brana com tensor energia-momento $T_{\mu \nu}$, podem ser escritas como:

$$
K_{\mu v}=\frac{\kappa_{5}}{6}\left(3 T_{\mu v}-h_{\mu v} T\right),
$$

onde

$$
K_{\mu \nu}=e_{\mu}^{A} e_{v}^{B} \nabla_{A} n_{B}
$$

é a curvatura extrínseca do espaço-tempo 4-dimensional, $\kappa_{5}$ é a constante de acoplamento gravitacional em 5 dimensões ${ }^{5}$, e $T$ é o traço de $T_{\mu \nu}$.

Não equivalentemente à [25], Creek et al. adotaram um tensor energia momento na brana em sua forma geral para um fluido perfeito com densidade de energia $\rho(t, r)$ e pressão $p(t, r)$ :

$$
T_{\mu \nu}=[\rho(t, r)+p(t, r)] h_{\mu \alpha} h_{v \beta} u^{\alpha} u^{\beta}+p(t, r) h_{\mu v},
$$

com $h_{\mu v}$ definido em (2.30). O vetor $u^{\mu}$ é a quadrivelocidade do fluido — paralela ao vetor do tipo tempo $T^{\mu}$ - que satisfaz a seguinte condição de normalização: $u^{\mu} u^{v} h_{\mu v}=-1$. Os autores escreveram a densidade de energia e a pressão numa equação de estado dada por

$$
p(t, r)=\frac{[v(t, r)-2]}{3} \rho(r, t),
$$

sendo que tal equação de estado é diferente da equação padrão — onde a pressão é uma grandeza proporcional à densidade de energia —, sem restringir de forma alguma $p$, pois $v$ é uma função arbitrária de $t$ e $r$.

A partir do tensor energia momento (2.33), substituindo-o em (2.31), as componentes de $K_{\mu \nu}$ tornaram-se:

$$
\begin{aligned}
K_{t t} & =-n\left(\ddot{\chi}+U r \chi^{\prime} \dot{\chi}^{2}-\frac{1}{2} U U^{\prime} \chi^{\prime}\right)=-\frac{\kappa_{5}}{6} \rho v\left(-U+r^{2} \dot{\chi}^{2}\right), \\
K_{r r} & =-n\left(\chi^{\prime \prime}+\frac{2 \chi^{\prime}}{r}+\frac{U^{\prime} \chi^{\prime}}{2 U}+U r \chi^{\prime 3}\right)=\frac{\kappa_{5}}{6} \rho\left[\frac{1}{U}+r^{2} \chi^{\prime 2}+\frac{(1+v) r^{4} \dot{\chi}^{2} \chi^{\prime 2}}{U-r^{2} \dot{\chi}^{2}}\right], \\
K_{t r} & =-n\left(\dot{\chi}^{\prime}+\frac{\dot{\chi}}{r}+U r \chi^{\prime 2} \dot{\chi}-\frac{U^{\prime} \dot{\chi}}{2 U}\right)=-\frac{\kappa_{5}}{6} \rho v r^{2} \dot{\chi} \chi^{\prime}, \\
K_{\theta \theta} & =-n\left(U r \chi^{\prime} \operatorname{sen}^{2} \chi-\operatorname{sen} \chi \cos \chi\right)=\frac{\kappa_{5}}{6} \rho r^{2} \operatorname{sen}^{2} \chi .
\end{aligned}
$$

\footnotetext{
${ }^{5} \kappa_{5}=8 \pi G_{5}$ e $G_{5}$ é a constante gravitacional em 5 dimensões.
} 
Além dessas equações, mais duas outras não independentes foram fornecidas pela conservação do tensor energia-momento. Foram elas

$$
\dot{\chi}\left(1+\frac{1}{2} U^{\prime} r-U+\frac{\kappa_{5} \rho}{6 n}(1+v) r^{2} \cot \chi\right)=-\frac{\kappa_{5} \dot{\rho}}{6 n} r^{2}
$$

e

$$
\chi^{\prime}\left(1+\frac{1}{2} U^{\prime} r-U+\frac{\kappa_{5} \rho}{6 n}(1+v) \frac{r^{4} \dot{\chi}^{2} \cot \chi}{r^{2} \dot{\chi}^{2}-U}\right)=-\frac{\kappa_{5} \rho^{\prime}}{6 n} r^{2} .
$$

Portanto, nesse modelo, uma brana quadridimensional, com um tensor energia momento que descreve um fluido perfeito, estará imersa num espaço-tempo tempo 5-dimensional com simetria esférica desde que tenhamos $\rho(t, r), p(t, r)$ e $\chi(t, r)$ consistentes com as equações (2.35)-(2.40).

Para uma simplificação considerável ocorrer, os autores assumiram que a trajetória da brana no bulk não depende do tempo. Sendo assim, $\dot{\chi}=0$, o que implica por (2.39) em $\dot{\rho}=0$. As equações remanescentes tornaram-se

$$
\begin{gathered}
v=-\left(\alpha^{\prime} r-\alpha\right)\left[\frac{\frac{1}{2} U^{\prime} r}{U\left(\alpha^{\prime} r-\alpha\right)+\alpha}\right], \\
U r^{2} \alpha^{\prime \prime}+\left(\alpha^{\prime} r-\alpha\right)\left(\frac{1}{2} U^{\prime} r-U\right)-\alpha=0,
\end{gathered}
$$

$\operatorname{com} \alpha=r \cos \chi$. As demais que restam, dependem de $\rho$ e suas derivadas:

$$
U\left(\alpha^{\prime} r-\alpha\right)+\alpha=\frac{\kappa_{5}}{6} \rho r \sqrt{U\left(\alpha^{\prime} r-\alpha\right)^{2}+r^{2}-\alpha^{2}}
$$

e

$$
\frac{\rho^{\prime} r}{\rho}=\left(\alpha^{\prime} r-\alpha\right)\left[\frac{1+\frac{1}{2} U^{\prime} r-U}{U\left(\alpha^{\prime} r-\alpha\right)+\alpha}\right] .
$$

Com a integração de (2.44), obtiveram

$$
\rho(r)=\frac{\rho_{0}}{r^{2}}\left[U\left(\alpha^{\prime} r-\alpha\right)+\alpha\right]
$$

onde $\rho_{0}$ é uma constante de integração. Substituindo-a em (2.43), escreveram esta última como

$$
U\left(\alpha^{\prime} r-\alpha\right)^{2}-\alpha^{2}+\left(1-\frac{36}{\kappa_{5}^{2} \rho_{0}^{2}}\right) r^{2}=0
$$

As equações (2.42) e (2.46), que expressam a variação de $\alpha$, puderam ser integradas com o 
auxílio de uma nova coordenada radial

$$
\tilde{r}=\int \frac{d r}{\sqrt{U}}
$$

que conduziu ao resultado

$$
\cos \chi=a e^{\tilde{r}}+b e^{-\tilde{r}}
$$

com $4 a b=1-36 / \kappa_{5}^{2} \rho_{0}^{2}$. Dessa forma, uma expressão para $v$ em (2.41) pôde ser determinada. Com isso, a partir de (2.34), igualmente uma expressão para $p(r)$ foi construída. Ambas equações - para $\rho(r)$ e $p(r)$ - em sua forma final foram escritas como:

$$
\begin{aligned}
& \rho(r)=\frac{\rho_{0}}{r}\left[\sqrt{U}\left(a e^{\tilde{r}}-b e^{-\tilde{r}}\right)+a e^{\tilde{r}}+b e^{-\tilde{r}}\right], \\
& p(r)=-\frac{2}{3} \rho_{0}(r)-\frac{\rho_{0} U^{\prime}}{6 \sqrt{U}}\left(a e^{\tilde{r}}-b e^{-\tilde{r}}\right) .
\end{aligned}
$$

Por fim, com a determinação de $T_{\mu \nu}$, foi possível escrever a métrica, com simetria esférica, induzida na brana como

$$
d s^{2}=-U d t^{2}+\frac{1-4 a b}{U\left(1-\alpha^{2}\right)} d r^{2}+\left(r^{2}-\alpha^{2}\right) d \Omega^{2} .
$$

Os autores de [43] utilizaram algumas métricas no bulk para o estudo desta abordagem (por (2.51) nota-se que fixando a métrica no bulk, ou seja, escolhendo $U$, fixa-se a métrica na brana). Entre as métricas 5-dimensionais, usaram a anti-de Sitter, Schwarzschild e Schwarzschild-AdS. Para este último caso, fixaram $U=1+k^{2} r^{2}-\mu / r^{2}$, sendo $k$ o inverso do raio AdS. Todo um estudo de trajetórias da brana no espaço-tempo 5-dimensional foi realizado. No fim, concluíram que para um bulk Schwarzschild-AdS o cenário seria mais realista, além de concordar com os modelos Randall-Sundrum que, segundo (2.17), requerem um bulk AdS.

Como pôde ser visto nesta seção, a abordagem a partir do bulk conduz a um conhecimento completo da geometria. Aqui, as métricas no bulk e brana são determinadas. Na próxima seção, a abordagem a partir da brana foi exposta. Como já dissemos, é a abordagem que usamos nesta tese e em nossos trabalhos.

\subsection{Soluções a partir da brana}

Com soluções a partir da brana, queremos dizer uma abordagem que parte da brana para construir uma solução de buraco negro ou buraco de minhoca no espaço-tempo quadridimen- 
sional sem especificar a solução total (bulk + brana). Em toda esta tese, como foram expostas nos capítulos 3 e 4, as métricas por nós obtidas partiram desta abordagem. Foram Shiromizu, Maeda e Sasaki os responsáveis pelo surgimento do caminho ilustrado nesta seção. Logo após a publicação dos modelos Randall-Sundrum, os três autores escreveram o trabalho The Einstein equations on the 3-brane world [26]. Nele, foram construídas as equações do campo gravitacional induzido numa brana - obtidas com auxílio das equações de Gauss-Codazzi e da assunção de uma simetria $\mathbb{Z}_{2}$ no bulk ${ }^{6}$. As equações induzidas relacionam o tensor de Einstein na brana com elementos no bulk:

$$
G_{\mu v}=-\Lambda_{4 D} g_{\mu v}+8 \pi G_{N} \tau_{\mu v}+\kappa_{5}^{2} \pi_{\mu v}-E_{\mu v}
$$

onde

$$
\begin{gathered}
\Lambda_{4 D}=\frac{1}{2} \kappa_{5}^{2}\left(\Lambda_{5 D}+\frac{1}{6} \kappa_{5}^{2} \lambda^{2}\right), \\
G_{N}=\frac{\kappa_{5}^{2} \lambda}{48 \pi}
\end{gathered}
$$

$\mathrm{e}$

$$
\pi_{\mu \nu}=-\frac{1}{4} \tau_{\mu \alpha} \tau_{\nu}^{\alpha}+\frac{1}{12} \tau \tau_{\mu \nu}+\frac{1}{8} g_{\mu \nu} \tau_{\alpha \beta} \tau^{\alpha \beta}-\frac{1}{24} g_{\mu \nu} \tau^{2}
$$

sendo $\Lambda_{4 D}, g_{\mu v}, \tau_{\mu \nu}$ e $\lambda$ a constante cosmológica, a métrica, o tensor energia momento e a tensão na brana ${ }^{7}$, respectivamente; $\Lambda_{5 D}$, como já dissemos, é a constante cosmológica no bulk; $\kappa_{5}^{2}$, como já vimos, é a constante de acoplamento gravitacional em 5 dimensões. O importante tensor $E_{\mu v}$ tem traço nulo $\left(E_{\mu}^{\mu}=0\right)$, é conhecido como a parte elétrica do tensor de Weyl - mede a influência do bulk na brana. Por fim, a constante $G_{N}$ é a constante gravitacional em 4 dimensões, que se relaciona com a mesma constante em 5 dimensões. Como já foi dito nas seções destinadas aos modelos Randall-Sundrum, todos os campos, exceto o gravitacional, estão confinados na brana, e as equações do campo gravitacional apresentadas reduzem-se às equações de Einstein da relatividade geral no limite de baixas energias.

Esse caminho, a resolução de (2.52), foi trilhado por alguns autores. Em [32], Dadhich et al. obtiveram uma solução do tipo Reissner-Nordström, com uma carga de maré induzida pelo bulk na brana. No trabalho [46], Gregory et al. supuseram uma equação de estado para um "fluido de Weyl", explorando características do tensor $E_{\mu \nu}$. Por outro lado, nos trabalhos [27, 28, 29, 30, 33, 35, 74], resolve-se a equação $R=$ cte para construir soluções na brana. É este último caso que abordamos nesta tese, especialmente os caminhos seguidos por Casadio

\footnotetext{
${ }^{6}$ No apêndice A, a dedução desse importante trabalho foi exposta.

${ }^{7}$ Suprimimos os índices no canto superior esquerdo em escales e tensores. Todas a grandezas $\left(R, R_{\mu v}, G_{\mu v}\right.$ etc.), doravante, referem-se à brana quadridimensional.
} 
et al. [27] e Bronnikov et al. [28], que tratam de simetria esférica, expostos no próximo capítulo, e por Aliev e Gümrükçüoglu [33], conforme o capítulo 4 dedicado à simetria axial.

Com a contração das equações induzidas com $g^{\mu \nu}$, sabendo que $G_{\mu \nu}=R_{\mu \nu}-\frac{1}{2} g_{\mu \nu} R$, temos

$$
R=4 \Lambda_{4 D}-8 \pi G_{N} \tau-\kappa_{5}^{2} \pi
$$

onde $R$ é o escalar de Ricci em 4 dimensões. A equação acima é uma equação diferencial para as componentes da métrica, e suas soluções podem conduzir a métricas com as simetrias axial ou esférica - buracos negros estáticos e em rotação e buracos de minhocas -, como veremos nos capítulos 3 e 4 .

Para o caso de simetria esférica, nós utilizamos, assim como [27, 28], o ansatz

$$
d s^{2}=-A(r) d t^{2}+\frac{1}{B(r)} d r^{2}+r^{2}\left(d \theta^{2}+\operatorname{sen}^{2} \theta d \phi^{2}\right),
$$

para resolver (2.56). Esse procedimento nos conduziu a uma família de soluções assintoticamente anti-de Sitter numa 3-brana, que generalizou os resultados de [28], publicadas no trabalho [29] em 2010 na revista Physical Review D, e a soluções assintoticamente de Sitter — buracos de minhoca — publicadas na mesma revista neste ano de 2012 [30]. No capítulo 3 , mostramos e comentamos esses dois trabalhos.

Para o caso axialmente simétrico mais geral, utilizamos o ansatz de Kerr-Schild, apresentado em [8]:

$$
d s^{2}=d s^{2}+H\left(l_{\mu} d x^{\mu}\right)^{2},
$$

onde $d s^{\prime 2}$ pode ser a métrica de Minkowski, ou anti-de Sitter, ou de Sitter; $H$ é uma função de $r$ e $\theta$, somente, e $l_{\mu}$ é um vetor tipo nulo ${ }^{8}$. A função $H$ é escolhida depender somente das variáveis $r$ e $\theta$, pois queremos uma métrica com simetria axial, ou seja, invariante por $t$ e $\phi$, simultaneamente. Segundo [68], no capítulo 32, o ansatz (2.58) - no caso de uma métrica assintoticamente plana - foi utilizado inicialmente por A. Trautman porque ele acreditava que ondas gravitacionais propagam informação, e isso pode ser conseguido se a métrica (2.58), nas formas covariante e contravariante, depender linearmente da mesma função $H(r, \theta)$. No capítulo 4, mostramos soluções por nós criadas com o ansatz de Kerr-Schild, seguindo e generalizando os resultados de Aliev e Gümrükçüoglu em [33]. Tais resultados mostraram-se assintoticamente de Sitter ou anti-de Sitter e foram expressos, depois de uma transformação de coordenadas, na forma (B.11), útil para o estudo de ótica geométrica de buracos negros em rotação, realizado no capítulo 5 .

Talvez, o maior problema da abordagem a partir da brana seja o desconhecimento da

\footnotetext{
${ }^{8}$ Aqui, estamos usando as coordenadas $(t, r, \theta, \phi)$.
} 
métrica total (bulk + brana). É justamente esse desconhecimento o alvo das principais críticas direcionadas a esse caminho. Segundo Seahra [47], como não conhecemos a métrica total, não sabemos se, por exemplo, a singularidade na brana estende-se pelo bulk. Também a forma do horizonte de eventos torna-se, segundo esse crítico, desconhecida, impossibilitando uma termodinâmica desses objetos. Entretanto, esse mesmo crítico, junto com Wesson, escreveu o trabalho [48], no qual aplica teoremas do tipo Campbell-Magaard a modelos como o RandallSundrum, com o intuito de determinar a métrica total (bulk + brana).

Os teoremas do tipo Campbell-Magaard, segundo o trabalho de Seahra e Wesson, partem do problema da imersão de um espaço-tempo $d$-dimensional numa variedade $(d+1)$ dimensional que satisfaz as equações de Einstein. A partir das equações de Gauss-Codazzi em $(d+1)$ dimensões, determinaram em cada foliação $d$-dimensional, $\Sigma_{\ell}$, a projeção de tais equações. Com isso, em cada hipersuperfície $\Sigma_{\ell}$ (uma brana, no caso do modelo RS), as equações do campo gravitacional são determinadas por três tensores:

$$
g_{\mu v}\left(x^{\mu}, \ell\right), K_{\mu v}\left(x^{\mu}, \ell\right) \text { e } E_{\mu v}\left(x^{\mu}, \ell\right) .
$$

O tensor $g_{\mu \nu}$ é a métrica induzida no espaço-tempo $d$-dimensional, $K_{\mu v}$ é a sua curvatura extrínseca, e o tensor $E_{\mu v}$ relaciona-se com o tensor de Riemann ${ }^{(d+1)} R_{A B C D}$ em $(d+1)$ dimensões por

$$
E_{\mu \nu} \equiv{ }^{(d+1)} R_{A B C D} n^{A} e_{\mu}^{B} n^{C} e_{v}^{D} .
$$

Os vetores $n^{A}$ e $e_{\mu}^{A}$ são normal e tangente à hipersuperfície $\Sigma_{\ell}$, respectivamente. O índice $\ell$ denota uma dada hipersuperfície; nesta, as coordenadas são dadas por $x^{\mu}$. Sendo simétricos, os tensores (2.59) têm cada um $d(d+1) / 2$ quantidades dinâmicas independentes. Portanto, o total de quantidades independentes a determinar em cada foliação $\Sigma_{\ell}$ é $n_{d i n}=3 d(d+1) / 2$. Como as equações que determinam o campo gravitacional não variam com a variação de $\ell$, tais equações são conservadas conforme se desloca de uma hipersuperfície a outra. Com isso, as equações que envolvem (2.59) são em $(d+1)$ dimensões interpretadas, na linguagem do formalismo hamiltoniano da relatividade geral, como equações que vinculam o sistema.

Então, a tarefa que Seahra e Wesson se colocaram foi construir uma solução para as equações de Einstein $(d+1)$-dimensionais tal que $\Sigma_{\ell}$ fosse uma foliação com propriedades geométricas apropriadas. Mas, a partir das equações de Gauss-Codazzi, os autores mostraram que na hipersuperfície $d$-dimensional existem $n_{v i n}=(d+1)(d+2) / 2$ equações independentes, chamadas de equações de vínculos. Para $d \geqslant 2$, o número de quantidades dinâmicas $n_{d i n}$ é maior do que $n_{v i n}$. Portanto, para $d \geqslant 2$ deve-se livremente determinar a dependência funcional de $n_{\text {livre }}=n_{\text {din }}-n_{\text {vin }}=d^{2}-1$ quantidades indeterminadas. "Esta liberdade é o 
coração do teorema de Campbell-Magaard", afirmam Seahra e Wesson", pois sendo " $n_{\text {livre }}$ maior do que o número de componentes independentes de $g_{\mu \nu}$, para $d \geqslant 2$, podemos escolher a métrica em $\Sigma_{0}$ corresponder a qualquer $d$-dimensional variedade lorentziana e ainda satisfazer as equações de vínculos". Portanto, completando seu esboço de prova, Seahra e Wesson concluem que para o teorema de Campbell-Magaard "qualquer d-dimensional variedade pode ser localmente imersa num espaço-tempo einsteiniano $(d+1)$-dimensional" 10 . Além disso, quando se escolhe a métrica induzida no espaço-tempo $d$-dimensional não se fixa de forma única a geometria em $(d+1)$ dimensões. Ou seja, pode-se imergir uma dada foliação $\Sigma_{0}$ em diferentes espaços einsteinianos. Dito de outra forma, em geral a geometria $(d+1)$-dimensional não é unicamente determinada pela geometria intrínseca de $\Sigma_{0}$.

${ }^{9}$ Cf. [48], p. 1327.

${ }^{10}$ Idem, Ibidem. Itálico dos autores. Doravante, todas as traduções são de minha autoria. 


\section{Capítulo 3}

\section{Simetria esférica em mundos brana}

Este capítulo aborda soluções com simetria esférica das equações do campo gravitacional induzido num contexto de mundos brana. Na primeira seção, elencamos dois caminhos percorridos nesse contexto: o de Casadio et al. e o de Bronnikov et al.. Na seção seguinte, expusemos a família de soluções assintoticamente anti-de Sitter (AdS) numa 3-brana sem conteúdo de matéria — os resultados dessa seção referem-se ao nosso Black holes and wormholes in AdS branes, publicado na revista Physical Review D em 2010 [29]. Em tal artigo, as soluções construídas referem-se a buracos negros e buracos de minhoca. Em seguida, soluções no mesmo contexto são apresentadas, com a diferença de que, nesse caso, tais soluções são interpretadas como buracos de minhocas numa brana assintoticamente de Sitter (dS). Recentemente, um trabalho nosso com o título Wormholes in de Sitter branes [30] foi publicado na mesma revista com estes últimos resultados. Por fim, na última seção, um estudo de geodésicas equatoriais foi feito para as soluções dS.

\subsection{Soluções assintoticamente planas}

Em New black holes in the brane world? [27], Casadio, Fabbri e Mazzacurati buscaram soluções com simetria esférica na brana, num contexto do tipo Randall-Sundrum [17, 18], propondo duas famílias de soluções que violam a igualdade $A(r)=B(r)$ no ansatz ${ }^{1}$ (2.57). Ambas famílias foram parametrizadas pela massa ADM e pelo parâmetro pós-newtoniano ${ }^{2}$

\footnotetext{
${ }^{1}$ Com isso, mostra-se a não aplicabilidade do teorema de Birkhoff no contexto de mundos brana. Na relatividade geral, por outro lado, esse teorema afirma que a única solução no vácuo com simetria esféria é a solução de Schwarzschild.

${ }^{2}$ A chamada parametrização pós-newtoniana (PPN), de acordo com Clifton et al., "é a ideia de criar uma construção que englobe um leque de diferentes teorias da gravidade e contém parâmetros que podem ser vinculados por observações (...)". De tal forma "que experimentais podem aplicar seus resultados para vincular teorias sem precisar conhecê-las em detalhes, e teóricos podem vincular suas novas teorias a fim de compará-las com os limites dos parâmetros pós-newtonianos já estabelecidos (...)”. Cf. [21], p. 20.
} 
$\beta$. O procedimento usado por esses autores foi, de forma análoga, resolver a equação (2.56), para os termos da métrica, assumindo a ausência de uma constante cosmológica na brana e vácuo na mesma. Ou seja, a equação citada torna-se simplificada:

$$
R=0
$$

Com o ansatz esfericamente simétrico, substituíram-no na equação (3.1), de tal forma a obter a seguinte equação diferencial:

$$
\frac{2}{r^{2}}(1-B)-B\left[\frac{A^{\prime \prime}}{A}-\frac{A^{\prime 2}}{2 A^{2}}+\frac{A^{\prime}(B)^{\prime}}{2 A B}+\frac{2}{r}\left(\frac{A^{\prime}}{A}+\frac{(B)^{\prime}}{B}\right)\right]=0,
$$

onde o símbolo linha significa derivada ordinária em relação à coordenada $r$, e soluções distintas surgiram de acordo com a escolha das funções $A(r)$ ou $B(r)$.

Nesta breve revisão do trabalho de Casadio et al., vamos apenas citar a família de soluções que parte da seguinte escolha:

$$
A(r)=1-\frac{2 M}{r} .
$$

Tal função $A(r)$ escolhida é a mesma da solução de Schwarzschild no contexto da relatividade geral. Entretanto, substituindo-a em (3.2), proveu a função

$$
B(r)=\frac{\left(1-\frac{3 M}{2 r}\right)}{\left(1-\frac{2 M}{r}\right)\left[1-\frac{M}{2 r}(4 \beta-1)\right]},
$$

onde, como já dissemos, $\beta$ é um parâmetro pós-newtoniano (experimentalmente, segundo [21], muito próximo da unidade) que torna $A(r)=B(r)$ quando assume valor 1. Com isso, (3.3) e (3.4) juntas fixaram a métrica na brana.

Com o uso de outro parâmetro na métrica, $\bar{\eta}=(4 / 9) \eta$, sendo $\eta=3(\beta-1)$ mais um parâmetro de PPN diferente de zero, os autores dividiram essa família de soluções em dois casos: $\bar{\eta}<0$ e $\bar{\eta}>0$. O mais interessante dos dois, quando $\bar{\eta}>0$, mostrou-se ser livre de singularidades e, segundo os autores, ao contrário de corda negra de Chamblin, Hawking e Reall [25], pode comportar um bulk não singular.

Num trabalho na mesma linha, General class of brane-world black holes [28], Bronnikov, Melnikov e Dehnen construíram uma classe geral de geometrias estáticas com simetria esférica, no interessante cenário que surgiu devido ao seminal trabalho de Lisa Randall e Raman Sundrum [17]. Usando as equações do campo gravitacional, deduzidas por Shiromizu et al. [26], esses autores obtiveram geometrias numa 3-brana sem conteúdo de matéria, fixando $A(r)$ - uma função suave escolhida de tal forma a anular-se em algum $r=r_{+}>0$ - para 
obter $B(r)$. Tal como Casadio et al., resolveram (3.1), impondo ainda que o escalar $R$ fosse uma função de $r$. Com a definição $f(r) \equiv r B(r)$, (3.2) tornou-se

$$
A\left(4 A+r A^{\prime}\right) f^{\prime}+\left[r\left(2 A A^{\prime \prime}-A^{\prime 2}\right)+3 A A^{\prime}\right] f=2 A^{2}\left[2-r^{2} R(r)\right],
$$

cuja solução é dada pela integral

$$
f(r)=\frac{2 A e^{3 \Gamma}}{\left(4 A+r A^{\prime}\right)^{2}} \int\left(4 A+r A^{\prime}\right)\left[2-r^{2} R(r)\right] e^{-3 \Gamma} d r,
$$

onde

$$
\Gamma(r)=\int \frac{A^{\prime}}{\left(4 A+r A^{\prime}\right)} d r .
$$

A resolução da integral (3.6), a partir de uma função $A(r)$, que obedece as condições impostas, fornece uma constante de integração, um parâmetro $C$ diferenciador de tipos de soluções. Ou seja, "uma escolha genérica da função $A(r)$ conduz a uma família de soluções com um parâmetro que, num certo intervalo de $C$ (uma constante de integração), unifica buracos negros não extremos globalmente regulares com uma estrutura causal do tipo Kerr, buracos negros extremos e buracos de minhocas simétricos."3

Entre as geometrias assintoticamente planas geradas por essa abordagem, constam aquela já obtida por Casadio et. al em (3.3)-(3.4) e outras soluções assintoticamente planas. Mas nesse trabalho, existe uma interessante solução que motivou nosso artigo [30], exposto na seção 3.3. Esta última foi construída a partir da função $A(r)$ da métrica de-Sitter (dS) pura

$$
A(r)=1-\frac{r^{2}}{L^{2}},
$$

que foi usada para resolver a equação

$$
R=4 \Lambda_{4 D}=\frac{12}{L^{2}},
$$

onde $\Lambda_{4 D}$ é a constante cosmológica na brana, e $L$ é o raio dS (nesse caso assumindo constante cosmológica positiva na brana). A solução de (3.2), onde agora $R$ é dado por (3.9), forneceulhes

$$
B(r)=\left(1-\frac{r^{2}}{L^{2}}\right)\left[1+\frac{C}{r\left(2 L^{2}-3 r^{2}\right)^{3 / 2}}\right] .
$$

Para a métrica fixada pelas funções (3.8) e (3.10), Bronnikov et al., afirmaram que nessa

\footnotetext{
${ }^{3}$ Cf. [28], p. 2.
} 
situação “(...) a única solução bem comportada é a de Sitter (...)." torna $A(r)=B(r)$. Como mostramos na seção 3.3, essa afirmação é equivocada. Para a mesma função (3.8), construímos soluções de buracos de minhoca nesse contexto.

\subsection{Soluções assintoticamente AdS}

A partir da vereda aberta por Casadio et al. e Bronnikov et al., generalizamos seus resultados, impondo constante cosmológica negativa ou positiva na brana. À constante cosmológica positiva a seção seguinte, que é fruto do nosso trabalho [30], foi dedicada. À constante cosmológica negativa esta seção, que mostra os resultados de um trabalho publicado por nós [29] em 2010, foi destinada.

As equações do campo gravitacional induzido, apresentadas no capítulo anterior (2.52), numa 3-brana sem conteúdo de matéria tomam uma forma mais simples devido a esta assunção. Isto é,

$$
G_{\mu v}=-\Lambda_{4 D} g_{\mu v}-E_{\mu v}
$$

pretende descrever o campo gravitacional numa 3-brana vazia. A constante $\Lambda_{4 D}$ e $g_{\mu \nu}$, como já foi dito, são a constante cosmológica e a métrica na brana, respectivamente. O tensor $E_{\mu \nu}$, conhecido como a parte elétrica do tensor de Weyl, devido à sua construção tem traço nulo $\left(E_{\mu}^{\mu}=0\right)$. Assim, por (2.56), a equação resolvida foi

$$
R=4 \Lambda_{4 D}=-\frac{12}{L^{2}},
$$

onde $R$ representa o escalar de Ricci na brana, e $L$ é o raio anti-de Sitter da mesma. Com o auxílio do ansatz (B.13), escrito nas coordenadas $(t, r, \theta, \phi)$, a equação (3.12) apresentou-se como

$$
\frac{2}{r^{2}}(1-B)-B\left[\frac{A^{\prime \prime}}{A}-\frac{A^{\prime 2}}{2 A^{2}}+\frac{A^{\prime} B^{\prime}}{2 A B}+\frac{2}{r}\left(\frac{A^{\prime}}{A}+\frac{B^{\prime}}{B}\right)\right]=4 \Lambda_{4 D} .
$$

Para resolver a equação acima, fixamos uma função $A$, levando-se em consideração sua analiticidade no horizonte, como fez Bronnikov et al. [28], de tal forma a obter como soluções da equação (3.13) uma família de soluções $\mathscr{B}$, tal que a função $B \in \mathscr{B}$. Nessa família, não temos somente $A=B$. Isto implica — como nos trabalhos citados na seção anterior — na não aplicabilidade do teorema de Birkhoff no contexto de mundos brana. Em nosso trabalho também propusemos o relaxamento desse teorema. A família de soluções construída possui membros que se distinguem entre si quando fixamos a constante de integração $C$. Dessa

\footnotetext{
${ }^{4}$ Cf. [28], p. 7.
} 
forma, existe um valor de $C\left(C=C_{0}\right)$ que torna $A=B$. Então, para realizar este trabalho, assumimos as seguintes condições:

Condição primeira: vácuo na brana ou, de forma equivalente, um tensor energiamomento efetivo com traço nulo.

Condição segunda: estaticidade e simetria esférica.

Condição terceira: existe um valor de $C\left(C=C_{0}\right)$ para que tenhamos $A=B$. Ou seja, esta solução, indicada por $\left(A_{0}, B_{0}\right)$, pertence a uma família $\mathscr{B}$ tal que seus membros são deformações contínuas de $\left(A_{0}, B_{0}\right)$.

A condição primeira nos diz que (3.11) é a equação do campo gravitacional; a segunda nos conduz à equação (3.13). Com a condição terceira em (3.13), chegamos à equação diferen$\mathrm{cial}^{5}$

$$
r^{2} A^{\prime \prime}+4 r A^{\prime}+2 A=2+\frac{12 r^{2}}{L^{2}}
$$

cuja solução mais geral — aquela que escolhemos e usamos —é:

$$
A(r)=A_{0}(r)=1-\frac{2 M}{r}+\frac{Q^{2}}{r^{2}}+\frac{r^{2}}{L^{2}},
$$

onde $M$ e $Q$ são constantes de integração, e $L$ está relacionada com a constante cosmológica na brana por: $L^{2}=-3 / \Lambda_{4 D}$. A função $A(r)$ escolhida foi, então, a mesma da métrica Reissner-Nordström-anti-de Sitter. Entretanto, $Q$ não foi interpretada como uma carga coulombiana no sentido usual. A condição primeira afirma que (3.11) diz respeito a uma brana vazia $^{6}$ ou, de forma equivalente, a um espaço-tempo 4-dimensional, no contexto da relatividade geral, com um tensor energia-momento efetivo com traço nulo. Assim, $Q$, quando tratamos de mundos brana, pode ser encarada como uma carga induzida pelo bulk na brana. Isso ocorre da mesma forma com geometrias que possuem outras simetrias (por exemplo, aquelas descritas no próximo capítulo). Por outro lado, quando pensamos que (3.11) é a equação de Einstein, $E_{\mu \nu}$ pode ser visto como um tensor energia-momento efetivo não nulo, e $Q$ pode ser interpretada como a medida de um campo de matéria na brana. A outra constante, $M$, tem a mesma interpretação de massa no sentido da métrica Reissner-Nordström-anti-de Sitter.

Os zeros da função $A(r)$ foram relevantes para um estudo da estrutura causal das geometrias construídas. Quando $L^{2}>0, M>0$ e $Q=0$, a função $A(r)$ tem somente um zero real e positivo, $r_{+}$. Quando $Q \neq 0$, temos um resultado um pouco maior. Existe, então, um valor máximo para $Q\left(Q_{\max }\right)$ que torna possível $A(r)$ ter zeros reais e positivos. Nesse caso, quando

\footnotetext{
${ }^{5}$ A equação (5) do nosso trabalho [29] tem um sinal errado, como pode-se notar comparando-a com (3.14).

${ }^{6}$ Para que a brana seja vazia, impusemos $\tau_{\mu v}=0$ na equação do campo gravitacional induzido, cf. eq. (A.33).
} 
$L^{2}>0, M>0$ e $Q^{2}>0$, a função $A(r)$ tem: (a) dois zeros reais simples $\left(r_{-}\right.$e $\left.r_{+}\right)$quando $|Q|$ é um pouco menor do que $Q_{\max }$, onde $Q_{\max }^{2} / r_{+}^{2}+3 r_{+}^{2} / L^{2}=0$; (b) um zero duplo positivo quando $|Q|=Q_{\max }$; (c) nenhum zero real se $|Q|>Q_{\max }$. Se $M \leq 0$, a função $A(r)$ não tem zeros reais e positivos, o que implica na possibilidade de termos soluções com singularidade nua. Em nosso trabalho, consideramos somente o caso $M>0$.

Como (3.13) é linear em termos de $B$, uma linear combinação de soluções, com uma função $A$ fixa, também é solução. Como estamos interessados em deformações de (3.15), assumimos o seguinte ansatz: a função $A(r)$ dada por (3.15) e a função $B(r)$ escrita como

$$
B(r)=B_{0}(r)+\left(C-C_{0}\right) B_{l i n}(r),
$$

com $\partial B_{\text {lin }} / \partial C=0$ e $B_{0}(r)=A_{0}(r)$. Usamos (3.15) e (3.16) para reescrever o vínculo (3.13) como uma equação diferencial ordinária de primeira ordem em $B_{l i n}$. Ou seja,

$$
h(r) \frac{d B_{l i n}(r)}{d r}+f(r) B_{l i n}(r)=0,
$$

com as funções $h(r)$ e $f(r)$ dadas por:

$$
h(r)=4 A_{0}(r)+r A_{0}(r)^{\prime}
$$

e

$$
f(r)=\frac{4 A_{0}(r)}{r}+4 A_{0}(r)^{\prime}+2 r A_{0}(r)^{\prime \prime}-\frac{r\left(A_{0}(r)^{\prime}\right)^{2}}{A_{0}(r)} .
$$

Com a escolha da função $A(r)$, dada por (3.15), chegamos como solução de (3.17), isto é, de (3.16), a uma família $\mathscr{B}$ de funções escrita como

$$
B(r)=A_{0}(r)\left[1+\left(C-C_{0}\right) \frac{P(r)}{\left(r-r_{0}\right)^{k}}\right],
$$

onde

$$
P(r)=\frac{L^{4} \exp \left[-\frac{K}{\sqrt{4 q-p^{2}}} \arctan \left(\frac{2 r+p}{\sqrt{4 q-p^{2}}}\right)\right]}{18\left(r-r_{0-}\right)^{k_{-}}\left(r^{2}+p r+q\right)^{2-\left(k+k_{-}\right) / 2}},
$$

sendo $r_{0}$ e $r_{0-}\left(r_{0}>r_{0-}\right)$ zeros reais e positivos da função $h(r)$. As constantes $p, q, k, k_{-}$e $K$ são definidas como

$$
\begin{gathered}
p=r_{0}+r_{0-}, q=\frac{L^{2} Q^{2}}{3 r_{0} r_{0-}}, \\
k=2-\frac{r_{0}^{4}+L^{2} M r_{0}-L^{2} Q^{2}}{r_{0}\left(r_{0}-r_{0-}\right)\left(r_{0}^{2}+p r_{0}+q\right)},
\end{gathered}
$$




$$
k_{-}=2+\frac{r_{0-}^{4}+L^{2} M r_{0-}-L^{2} Q^{2}}{r_{0-}\left(r_{0}-r_{0-}\right)\left(r_{0-}^{2}+p r_{0-}+q\right)}
$$

$\mathrm{e}$

$$
K=2\left[q\left(\frac{k}{r_{0}}+\frac{k_{-}}{r_{0-}}\right)-p\left(2-\frac{k+k_{-}}{2}\right)\right] .
$$

A família de geometrias indicada em (3.15) e (3.20) é rotulada pela constante de integração $C(C \in \mathbb{R})$ da equação diferencial de primeira ordem resolvida. Podemos, então, deformar a solução usual, onde $A(r)=B(r)$, usando a constante $C$ para obter uma geometria qualquer da família tão próxima ou tão distante da solução usual. Para um importante valor da constante de integração $\left(C=C_{0}\right)$, valor este que iguala $B(r)$ à função $A(r)$ e conduz à solução ReissnerNordström-anti-de Sitter $(Q \neq 0)$ ou Schwarzschild-anti-de Sitter $(Q=0)$, escrevemo-lo em termos de $r=r_{+}$. Em termos de $r_{+}$, que foi interpretado como o raio do horizonte de eventos ou raio do horizonte de Killing, como fica claro logo a seguir, tal parâmetro foi expresso por

$$
C_{0}=\frac{\left(r_{+}-r_{0}\right)^{k}}{P\left(r_{+}\right)}
$$

Deve-se enfatizar que quando $M>0$ e $L^{2}>0$, a situação por nós estudada: (a) $f(r)$ terá dois zeros reais positivos se $0<Q<Q_{\max }$ ou terá um zero se $Q=0$; (b) $0 \leq r_{0-} \leq r_{-} \leq$ $r_{0} \leq r_{+}$; (c) $4 q-p^{2}>0$, o que evita uma raiz imaginária em (3.21). Portanto, $P(r)$ é positivo definido, e as funções $A(r)$ e $B(r)$ são bem comportadas quando $r>r_{0}$.

As importantes constantes $r_{0}$ e $r_{+}$podem ser escritas em termos de $M, Q$ e $L$, embora tais expressões sejam grandes demais para serem escritas aqui. Para simplificá-las, fizemos $Q=0$ para ter

$$
\frac{r_{+}}{L}=\left[\frac{M}{L}+\sqrt{\left(\frac{M}{L}\right)^{2}+\frac{1}{27}}\right]^{1 / 3}-\frac{1}{3}\left[\frac{M}{L}+\sqrt{\left(\frac{M}{L}\right)^{2}+\frac{1}{27}}\right]^{-1 / 3} .
$$

O polinômio $f(r)$ nesse caso tem somente a raiz $r_{0}$, que escrevemos como:

$$
\frac{r_{0}}{2^{-1 / 3} L}=\left[\frac{M}{L}+\sqrt{\left(\frac{M}{L}\right)^{2}+\frac{32}{729}}\right]^{1 / 3}-\left(\frac{32}{729}\right)^{1 / 3}\left[\frac{M}{L}+\sqrt{\left(\frac{M}{L}\right)^{2}+\frac{32}{729}}\right]^{-1 / 3} .
$$

Notamos também que $p \rightarrow r_{0}, q \rightarrow L^{2} / 3 r_{0}, r_{0-} \rightarrow 0$ e $k_{-} \rightarrow 0$, quando $Q \rightarrow 0$.

As três condições estabelecidas mais as funções $A(r)$ e $B(r)$, dadas por (3.15) e (3.20), 
respectivamente, definem um tensor energia-momento efetivo:

$$
\left(T^{e f}\right)_{v}^{\mu}=-\frac{E_{v}^{\mu}}{8 \pi}=\left(\begin{array}{cccc}
-\rho^{e f} & & & \\
& p_{\text {rad }}^{e f} & & \\
& & p_{\text {tan }}^{e f} & \\
& & & p_{\text {tan }}^{e f}
\end{array}\right) .
$$

As expressões para a densidade e pressões efetivas são:

$$
\begin{aligned}
-8 \pi \rho^{e f} & =-E_{t}^{t}=-\frac{3}{L^{2}}+\frac{B-1}{r^{2}}+\frac{B^{\prime}}{r} \\
8 \pi p_{\text {rad }}^{e f} & =-E_{r}^{r}=-\frac{3}{L^{2}}+\frac{B-1}{r^{2}}+\frac{A^{\prime} B}{r A} \\
8 \pi p_{\text {tan }}^{e f} & =-E_{\theta}^{\theta}=-E_{\phi}^{\phi} \\
& =+\frac{3}{L^{2}}-\frac{B-1}{r^{2}}-\frac{B^{\prime}}{2 r}-\frac{A^{\prime} B}{2 r A}
\end{aligned}
$$

Então, por construção, as geometrias dadas por $A(r)$ e $B(r)$ são soluções das equações do campo gravitacional (3.11) com um tensor energia-momento efetivo, cujas componentes são dadas por (3.30)-(3.32). Pode-se notar que a condição $E_{\mu}^{\mu}=0$ é satisfeita. É interessante frisar que $\rho^{e f}, p_{\text {rad }}^{e f}$ e $p_{\text {tan }}^{e f}$ são finitas quando $r>r_{0}$. Este detalhe será importante mais tarde.

A seguir, consideramos extensões maximais lorentzianas ${ }^{7}$ das soluções criadas. Tais extensões nos conduziram a diagramas de Penrose $^{8}$ da família de geometrias. A constante $C$ mostrou-se importante pois determinados valores desse parâmetro fazem com que membros da família diferenciem-se. Ou seja, a família de soluções possui tipos de geometrias diferentes, que podem ser fisicamente interpretadas como buracos negros ou buracos de minhoca. Em todos casos, o comportamento dos membros da família é o mesmo quando $r$, a coordenada radial, é grande. Quando $r$ tende ao infinito, a função $P(r)$ tende a zero, e as geometrias apresentam um comportamento assintótico semelhante à geometria anti-de Sitter pura. Isto é,

$$
B(r)=A(r)=1+\frac{r^{2}}{L^{2}}+\mathscr{O}\left(\frac{1}{r}\right),
$$

quando $r \rightarrow \infty$. Por outro lado, fora dessa condição, para valores de $r$ finitos e não muito

\footnotetext{
${ }^{7}$ Extensões lorentzianas referem-se a métricas lorentzianas. Espaços-tempos de interesse na relatividade geral são descritos por métricas desse tipo. Tais métricas, quando escritas na forma canônica, ou seja, diagonalizadas, têm pelo menos um termo negativo em sua diagonal principal (ou pelo menos um positivo, dependendo da convenção). Por outro lado, métricas euclidianas têm todos membros positivos em sua diagonal principal, cf. [50], p. 73.

${ }^{8}$ Diagramas de Penrose ou diagramas conforme, de uma forma poética e pouco rigorosa, expressam o infinito no finito. Trazem-nos a estrutura causal de um espaço-tempo, cf. apêndice C.
} 
grandes, a estrutura dos membros da família depende fortemente de $B(r)$, ou seja, de $C$. Os zeros de $B(r)$ têm, assim, muita importância na tarefa de construção dos diagramas. O maior zero de $A(r)$, que escrevemos como $r_{+}$, é também o maior zero de $B(r)$, quando $C \geq 0$ (único zero, se $C \geq C_{0}$, por (3.20)). Isto implica que as métricas consideradas, nas coordenadas $(t, r, \theta, \phi)$, são bem definidas no intervalo $r>r_{+}$, quando $C \geq 0$. As extensões maximais foram nesses casos buracos negros. Quando $C<0$, a extensão maximal nos conduziu a uma interpretação de buraco de minhoca. Neste último caso, existe um zero $r_{t h r}$, na função $B(r)$, tal que $r_{t h r}>r_{+}$.

Como $A(r)$ e $B(r)$ têm zeros em $r=r_{+}$, sendo o maior zero de ambas as funções (no caso $C \geq 0$ ), $r_{+}$é um candidato a horizonte de eventos (mesmo não sendo um horizonte de eventos, em todos os casos explorados aqui, elencados logo a seguir, $r_{+}$sempre é o raio do horizonte de Killing ${ }^{9}$ ). Isto porque o horizonte de eventos é uma superfície nula de raio constante, uma membrana de caminho único que protege a singularidade. Como mostramos a seguir, nem todas as soluções da família exposta apresentam uma singularidade física.

Para confeccionar os diagramas de Penrose, precisamos construir os chamados blocos ${ }^{10}$. Tais dividem as geometrias focadas em interior e exterior. É o horizonte de Killing que une os blocos interior e exterior, delimitando, assim, o espaço-tempo. Definimos a coordenada tartaruga, $r^{*}$, com a expressão

$$
\frac{d r^{*}}{d r}=\frac{1}{\sqrt{A(r) B(r)}} .
$$

Com isso, as coordenadas temporais avançada $(v)$ e retardada $(w)$ foram escritas nas formas tradicionais

$$
w=t-r^{*} \quad \mathrm{e} \quad v=t+r^{*} .
$$

A partir destas, definimos as coordenadas $W=\operatorname{arctg} w$ e $V=\operatorname{arctg} v$. E para terminar, a coordenada do tipo tempo $T$ e a coordenada do tipo espaço $X$ foram introduzidas. Com isso, a métrica do espaço-tempo, $g_{\mu v}\left(d s^{2}\right)$, foi escrita em termos de uma métrica conforme, $\hat{g}_{\mu v}\left(d \hat{s}^{2}\right)$ :

$$
\begin{gathered}
d \hat{s}^{2}=\Omega(T, X)^{2} d s^{2}, \\
d \hat{s}^{2}=-d T^{2}+d X^{2}+r(T, X)^{2}\left(d \theta^{2}+\operatorname{sen}^{2} \theta d \phi^{2}\right) .
\end{gathered}
$$

O espaço-tempo físico mais os limites singulares e infinitos são conformalmente mapeados numa variedade com uma fronteira — que é o chamado diagrama de Penrose. Então, o bloco exterior das geometrias é o mesmo da geometria anti-de Sitter, pois por (3.33) e (3.34) a

\footnotetext{
${ }^{9}$ Uma breve discussão sobre a diferença entre horizontes de eventos e de Killing encontra-se no apêndice C.

${ }^{10}$ Para um estudo sobre esses blocos, o trabalho de Martin Walker, Block diagrams and the extension of timelike two-surfaces, é uma boa referência; cf. [72].
} 
família de soluções tem um comportamento assintótico do tipo anti-de Sitter puro. Entretanto, temos até cinco tipos de blocos interiores distintos na família, ou seja, cinco tipos de diagramas de Penrose diferentes.

\section{Buracos negros singulares assintoticamente AdS}

Quando $C>C_{0}$, a função $B(r)$ tem um único zero, $r=r_{+}$. Como assumimos $M>0 \mathrm{e}$ $L^{2}>0$, temos a importante desigualdade: $0<r_{0}<r_{+}$. O comportamento das funções $A(r)$ e $B(r)$ neste caso e o diagrama de Penrose correspondente são exibidos na figura 3.1. De acordo com esta figura, $B(r)$ diverge em $r_{0}$. Isto é, (3.20) mostrou-nos que

$$
\lim _{r \rightarrow r_{0}^{+}} B(r)=-\frac{1}{\left(r-r_{0}\right)^{k}}+\mathscr{O}\left(r-r_{0}\right)^{0} \rightarrow-\infty \quad \text { quando } \quad r \rightarrow r_{0}
$$

Quando construímos o escalar de Kretschmann, $R_{\alpha \beta \gamma \delta} R^{\alpha \beta \gamma \delta}$, notamos que $r_{0}$ é a singularidade física ou real.

$$
\left|R_{\alpha \beta \gamma \delta} R^{\alpha \beta \gamma \delta}\right|=\frac{1}{\left(r-r_{0}\right)^{6}}+\mathscr{O}\left(r-r_{0}\right)^{0} \rightarrow \infty \text { quando } r \rightarrow r_{0} .
$$

De forma diferente às geometrias Schwarzschild-AdS e Reissner-Nordström-AdS, a singularidade nessa geometria, num contexto de mundos brana, não fica na origem do sistema de coordenadas, em $r=0$, quando se usa $(t, r, \theta, \phi)$, mas em $r=r_{0}$. Como consequência, as componentes do tensor energia-momento (3.30)-(3.32) divergem nesse ponto. Então, a máxima extensão da métrica foi no intervalo $r_{0}<r<\infty$. Colando os dois blocos $-\mathrm{o}$ interior, que nas coordenadas $(t, r, \theta, \phi)$ abrange $r_{0}<r<r_{+}$, e o exterior, que naquelas coordenadas abrange $r_{+}<r<\infty$-, temos o diagrama de Penrose do caso $C>C_{0}$ exposto na figura 3.1 à direita, semelhante ao Schwarzschild-AdS, exposto no apêndice C. Neste caso, $r_{+}$representa um horizonte de eventos, protegendo a singularidade física, situada em $r_{0}$.

Como um último comentário, quando $Q=Q_{\max }$ e $C \geq 0$, notamos que a estrutura causal mudou drasticamente. Nesse caso, $r_{+}=r_{0-}=r_{0}$, e $r_{+}$é um zero duplo da função $A(r)$ e um ponto que faz com que a função $B(r)$ e o escalar de Kretschmann divirjam. Nessa configuração, temos, então, singularidades nuas, não exploradas nesta tese.

\section{Buracos negros: Schwarzschild-AdS e Reissner-Nordström-AdS}

Quando $C=C_{0}$, temos as soluções de Schwarzschild-AdS $(Q=0)$ e Reissner-NordströmAdS $\left(Q \neq 0\right.$ com $\left.0<Q<Q_{\max }\right)$, seus diagramas de Penrose já são conhecidos ${ }^{11}$. No apên-

\footnotetext{
${ }^{11}$ Cf., por exemplo, [76].
} 
dice $\mathrm{B}$, ambas métricas foram expostas.
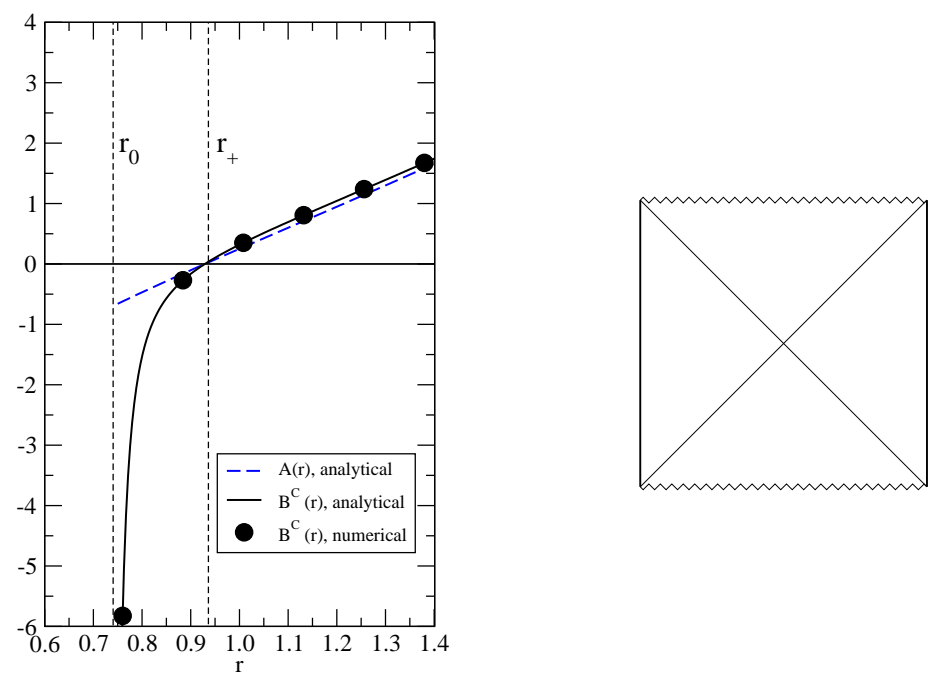

Figura 3.1: (Esquerda) Gráfico das funções $A(r)$ e $B(r)$ com $C>C_{0}$. Neste gráfico, $M=1, Q=$ $1 / 2, L=1, C=15$ e $C_{0}=10.32$. Como ilustração, a solução numérica de (3.13) para $B$ também é exibida. (Direita) Diagrama conforme da máxima extensão da solução com $C>C_{0}$. As linhas verticais representam o infinito, as diagonais representam as superfícies nulas (horizontes de eventos), e as irregulares, a singularidade.

\section{Buracos negros regulares assintoticamente AdS}

Quando $0<C<C_{0}$, temos buracos negros regulares assintoticamente AdS; neste caso, a função $B(r)$ tem um zero simples, $r_{\text {min }}$, dentro do intervalo $\left(r_{0}, r_{+}\right)$. Depois da extensão analítica da métrica, concluímos que a coordenada radial é válida somente para $r>r_{\text {min }}$. $\mathrm{O}$ bloco interior tem uma estrutura de buraco de minhoca ${ }^{12}$, como pode ser visto pela transformação de coordenadas $(t, r) \rightarrow(t, x)$, onde $x=r_{\min }+x^{2}$. O buraco negro para este caso não apresenta uma singularidade, $r_{+}$representa somente um horizonte de Killing. Casos como este já foram relatados nos trabalhos [27, 28]. O diagrama de Penrose desse tipo de solução é dado pela figura 3.2 à direita.

\section{Buracos negros extremos assintoticamente AdS}

Quando $C=0$, a função $B(r)$ tem um zero duplo em $r=r_{+}$e é positiva em todo intervalo $r_{0}<r<\infty$, como pode ser visto na figura 3.3. Para fazer a extensão além do horizonte, reescrevemos a métrica usando a coordenada quase-global $u$, que obedece a condição $g_{t t} g_{u u}=$

\footnotetext{
${ }^{12}$ Discutimos, logo a seguir, um pouco mais sobre buracos de minhoca, inclusive na próxima seção, dedicada somente a estes curiosos objetos.
} 
-1. Assim, a métrica com simetria esférica, na sua forma mais geral (B.13), ficou

$$
d s^{2}=-\mathscr{A}(u) d t^{2}+\frac{d u^{2}}{\mathscr{A}(u)}+r(u)\left(d \theta^{2}+\operatorname{sen}^{2} \theta d \phi^{2}\right)
$$

onde temos

$$
\mathscr{A}(u)=A(r), \quad r(u)=r, \quad \mathscr{A}(u)\left(\frac{d r}{d u}\right)^{2}=B(r) .
$$

A coordenada radial quase-global é conveniente no estudo de geometrias com simetria esférica $^{13}$. Ela traz para geometrias esféricas gerais as boas propriedades da coordenada $r$ da solução de Schwarzschild no contexto da relatividade geral. O nome quase-global deve-se ao motivo de que, próximo ao horizonte, essa coordenada varia como as bem comportadas coordenadas de Kruskal, que são usadas na extensão maximal da métrica de Schwarzschild.
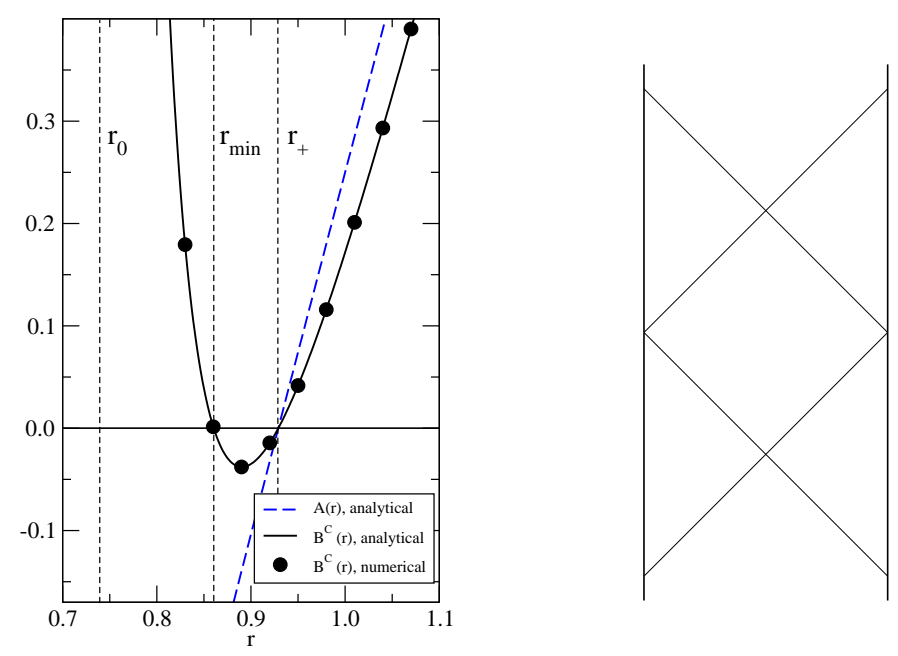

Figura 3.2: (Esquerda) Gráfico das funções $A(r)$ e $B(r)$ com $0<C<C_{0}$. Neste gráfico, $M=1$, $Q=1 / 2, L=1, C=5$ e $C_{0}=10.32$. Como ilustração, a solução numérica de (3.13) para $B$ também é mostrada. (Direita) Diagrama conforme da máxima extensão da solução com $0<C<C_{0}$. As linhas verticais representam o infinito, a diagonais as superfícies nulas (horizontes de Killing).

Para nos certificarmos de que a geometria com $C=0$ pode ser estendida, notamos que a função $\mathscr{A}(u)$ tem um zero duplo em $u=0$, onde $r(0)=r_{+}$:

$$
\mathscr{A}(u)=\frac{\left(2-r_{+} R\right)^{2}}{2 A^{\prime}\left(r_{+}\right) r_{+}^{4}} u^{2}+\mathscr{O}\left(u^{4}\right)
$$

A extensão interior somente foi possível pois $r(u)$ é analítica. Em particular, próximo ao

\footnotetext{
${ }^{13}$ Cf., por exemplo, [74].
} 
horizonte de Killing, $u=0$. Ou seja,

$$
r(u)=r_{+}+\frac{\left(2-r_{+} R\right)^{2}}{2 A^{\prime}\left(r_{+}\right)^{2} r_{+}^{4}} u^{2}+\mathscr{O}\left(u^{4}\right) .
$$

Nesse sistema de coordenadas $(t, u, \theta, \phi)$, a métrica tem a assinatura ${ }^{14}$ correta dentro do horizonte de Killing $(u<0)$, como pode ser visto em (3.40) e (3.42). Antes da mudança de coordenadas, pelo gráfico na figura 3.3, as funções $A(r)$ e $B(r)$ tinham sinais opostos na região interna $\left(r<r_{+}\right)$. Isto, de acordo com a forma geral de uma métrica com simetria esféria (B.13), conduzia a uma assinatura diferente da métrica dentro do horizonte. Com a adoção da coordenada quase-global esse problema foi resolvido. Assim, a máxima extensão lorentziana da métrica foi formada por duas regiões assintoticamente AdS $(u \rightarrow \infty, u \rightarrow-\infty)$ separadas por um horizonte de Killing $\left(u=0\right.$ ou $\left.r=r_{+}\right)$, de acordo com a figura 3.3 à direita.
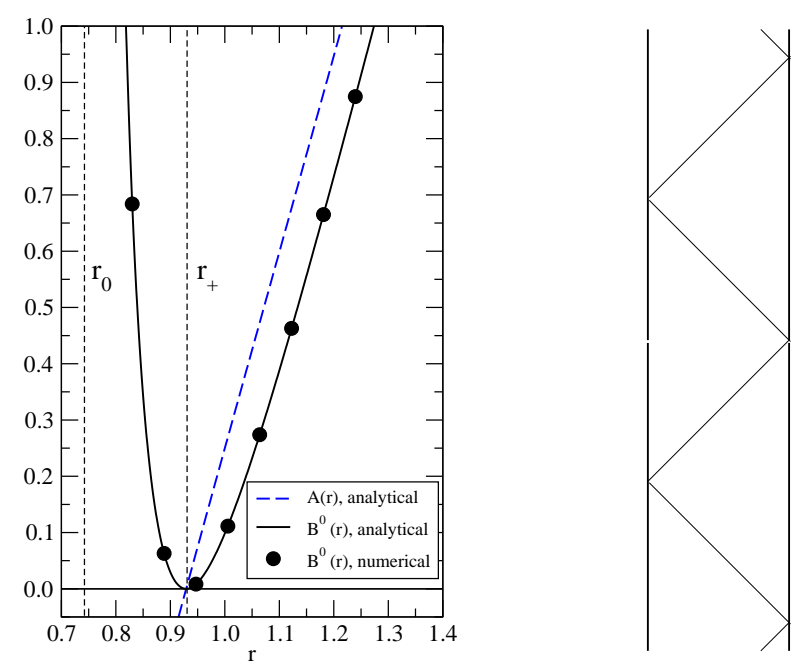

Figura 3.3: (Esquerda) Gráfico das funções $A(r)$ e $B(r)$ com $C=0$. Neste gráfico, $M=1, Q=$ $1 / 2, L=1, C=0$ e $C_{0}=10.32$. Como ilustração, a solução numérica de (3.13) para $B$ também é exibida. (Direita) Diagrama conforme da máxima extensão da solução $\operatorname{com} C=0$. As linhas verticais representam o infinito, a diagonais, as superfícies nulas (horizontes de Killing).

\section{Buracos de minhoca assintoticamente AdS}

Quando $C<0$, temos buracos de minhoca assintoticamente AdS. Buracos de minhoca têm ganho destaque nas pesquisas físicas desde o importante trabalho de Morris e Thorne, Wormholes in spacetime and their use for interstellar travel: A tool for teaching general relativity, em 1988 [10]. Nesse trabalho, os autores consideraram buracos de minhoca que

\footnotetext{
${ }^{14} \mathrm{~A}$ assinatura de uma métrica, quando escrita em sua forma canônica, é dada pelo numero de termos positivos e negativos na sua diagonal principal. Na métrica (B.13), por exemplo, temos um termo negativo $\left(g_{t t}\right)$ e três positivos $\left(g_{r r}, g_{\theta \theta}, g_{\phi \phi}\right)$. Nesse caso, a assinatura é +2 .
} 
possam ser tidos como pontes, estreitadores de caminho, entre duas regiões distantes do universo. O termo cunhado para tais buracos de minhoca é transitável, ou seja, estes objetos permitem que um “ homem (...) possa viajar de forma segura (...) num tempo razoável para poder retornar a tempo e contar sua façanha." 15 Para que seja transitável, um buraco de minhoca não pode ter um horizonte de eventos. Um horizonte de eventos impediria o retorno de um viajante ao seu ponto de partida. Tal objeto compacto, quando a métrica é lorentziana, é conhecido como buraco de minhoca lorentziano e transitável. Nesta seção e na próxima, lidamos apenas com esse tipo de buraco de minhoca.
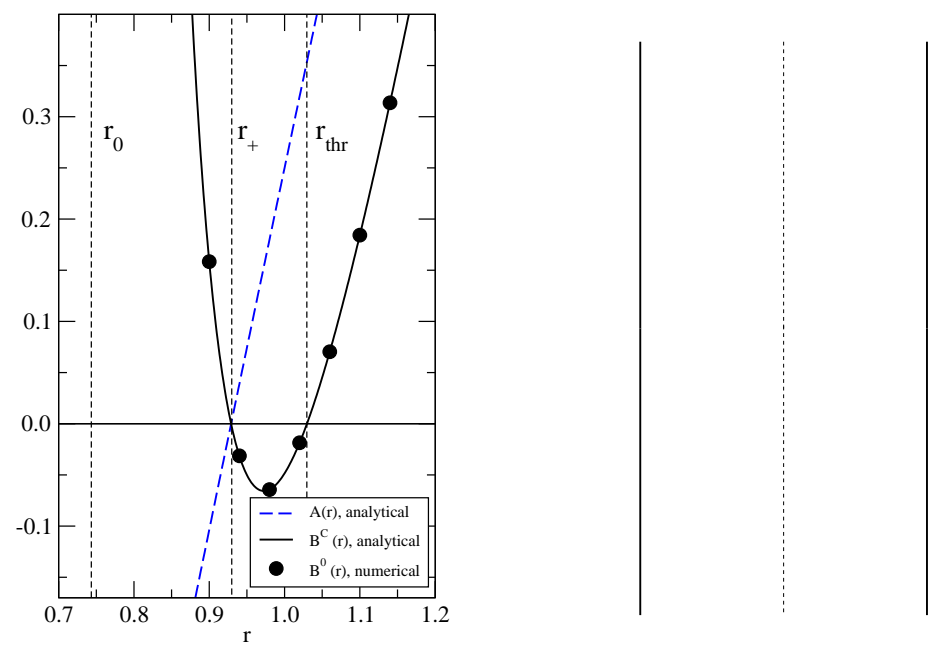

Figura 3.4: (Esquerda) Gráfico das funções $A(r)$ e $B(r) \operatorname{com} C<0$. Neste gráfico, $M=1, Q=1 / 2$, $L=1, C=-5$ e $C_{0}=10.32$. Como ilustração, a solução numérica de (3.13) para $B$ também é mostrada. (Direita) Diagrama conforme da máxima extensão da solução $\operatorname{com} C<0$. A linha pontilhada vertical significa a garganta do buraco de minhoca, e as linhas verticais representam o infinito.

Para um valor negativo da função $C$, a função $B(r)$ tem dois zeros simples e positivos $\left(r_{+}\right.$e $\left.r_{t h r}\right)$, onde $r_{+}<r_{t h r}$ e $A\left(r_{t h r}\right) \neq 0$. As coordenadas $(t, r, \theta, \phi)$ não são válidas para $r<r_{\text {thr }}$; adotando-se uma outra coordenada radial, $\ell$, chamada de comprimento próprio como pode ser visto, por exemplo, em [73, 74] —, temos

$$
\frac{d \ell(r)}{d r}=\frac{1}{\sqrt{B(r)}}
$$

Pode-se notar por (3.44) que a função $r(\ell)$ tem um mínimo $r_{t h r}$ em $\ell=0$ (este valor de $\ell$ é escolhido de tal forma que não se perde a generalidade com esta escolha). Como $r_{+}<$ $r_{t h r}$, o zero de $B(r), r_{+}$, não tem mais importância na estrutura causal neste caso - não há aqui um horizonte de eventos, temos, então, um buraco de minhoca transitável. Portanto, interpretamos $r_{t h r}$ como a garganta de um buraco de minhoca transitável que une duas regiões

\footnotetext{
${ }^{15}$ Cf. [73], p. 100. Nessa obra, um estudo detalhado sobre buracos de minhoca é feito.
} 
assintoticamente AdS, como mostra a figura 3.4 à direita. Os escalares e as componentes do tensor energia momento efetivo são finitos em todo espaço-tempo.

\subsection{Soluções assintoticamente dS}

Como já foi dito, no trabalho General class of brane-world black holes, Bronnikov, Melnikov e Dehnen afirmam que o procedimento de construção de soluções no contexto de mundos brana descrito na seção anterior conduz - para o caso de constante cosmológica positiva na brana - somente à solução de Sitter pura. Nesta seção, mostramos que esses autores se equivocaram. Conseguimos, no contexto mencionado, uma família de buracos de minhoca.

Aqui o caminho é o mesmo: a resolução das equações do campo gravitacional induzido na 3-brana deduzidas por Shiromizu et al., apresentadas no apêndice A. Para o caso de uma brana com constante cosmológica positiva e vazia, a equação (3.11), de acordo com a seção anterior, tornou-se

$$
R=4 \Lambda_{4 D}
$$

onde, como já dissemos, $R$ é o escalar de Ricci na brana, e $\Lambda_{4 D}$ é a constante cosmológica na mesma ${ }^{16}$. Quando usamos o ansatz (B.13) em (3.45), chegamos, como na seção anterior, à equação diferencial (3.13). Em nosso trabalho [30], propusemos construir espaços-tempo assintoticamente de Sitter, ou seja, espaços-tempo com $\Lambda_{4 D}>0$. Além disso, assumimos que estes são próximos à solução no vácuo com simetria esférica dada por

$$
A_{0}(r)=B_{0}(r)=1-\frac{2 M}{r}+\frac{Q^{2}}{r^{2}}-\frac{\Lambda_{4 D}}{3} r^{2},
$$

sendo $M$ e $Q$ constantes de integração positivas. Indicamos uma solução particular $(A, B)$ de funções que satisfazem o vínculo (3.13), de modo que esta faça parte de uma família de soluções $\mathscr{B}$. Ou seja, também neste caso, assumimos as três condições expostas na seção anterior. Como na seção anterior, os zeros de $A_{0}$ são de muita importância. Quando $M>0, Q \neq 0$ e $0<\Lambda_{4 D}<\Lambda_{e x t}$, onde

$$
\Lambda_{\text {ext }}=\frac{3}{8 Q^{2}}-\frac{1}{32}\left[\left(\frac{9 M^{2}}{Q^{3}}-\frac{6}{Q}\right)^{2}-3 M\left(\frac{9 M^{2}}{Q^{4}}-\frac{8}{Q^{2}}\right)^{3 / 2}\right]
$$

é o valor extremo de $\Lambda_{4 D}$, a função $A_{0}(r)$ tem quatro zeros reais $\left(r_{c}, r_{+}, r_{-}, r_{n}\right)$ que se relacionam da seguinte forma: $r_{n}<0<r_{-}<r_{+}<r_{c}$. No intervalo entre $r_{n}<r<r_{c}$, a

\footnotetext{
${ }^{16}$ Para que esta seção fique de acordo com nosso trabalho [30], escrevemos, cá como lá, o escalar de Ricci na brana somente como função da constante cosmológica, $\Lambda_{4 D}$, e não como função do raio dS, $L$.
} 
função $h(r)$ tem igualmente quatro zeros $\left(r_{0}, r_{0-}, r_{0--}, r_{0 n}\right)$ que obedecem a desigualdade $r_{0 n}<0<r_{0--}<r_{0-}<r_{0}$. Não expusemos as expressões de tantas raízes neste texto por serem exageradamente grandes. O que vale a pena destacar é que $r_{+}<r_{0}<r_{c}$ sempre quando $0<\Lambda_{4 D}<\Lambda_{\text {ext }}$.

Para resolver (3.13), impusemos $A(r)=A_{0}(r)$ e uma redefinição da função $B(r)$ :

$$
B(r)=B_{0}(r)-C B_{\text {lin }}(r)
$$

sendo $\partial B_{\text {lin }} / \partial C=0$ e $B_{0}(r)=A_{0}(r)$. Usamos, da mesma maneira nesta seção, (3.46) e (3.48) para reescrever o vínculo (3.13) como uma equação diferencial ordinária de primeira ordem em $B_{\text {lin }}$, que assumiu a forma (3.17) depois das substituições feitas.

A solução da equação diferencial ordinária (3.17), para a correção $B_{l i n}(r)$, foi

$$
B_{\text {lin }}(r)=A_{0}(r) \frac{\left(r-r_{0--}\right)^{c_{0--}}}{\left(r-r_{0}\right)^{c_{0}}\left(r-r_{0-}\right)^{c_{0-}}\left(r-r_{0 n}\right)^{c_{0 n}}},
$$

onde as constantes positivas $c_{0}, c_{0-}, c_{0--}$ e $c_{0 n}$ assumiram a forma

$$
\begin{gathered}
c_{0}=\frac{2}{\Lambda_{4 D}} \frac{r_{0}\left(2 \Lambda_{4 D} r_{0}^{2}-1\right)}{\left(r_{0}-r_{0-}\right)\left(r_{0}-r_{0--}\right)\left(r_{0}-r_{0 n}\right)}, \\
c_{0-}=-\frac{2}{\Lambda_{4 D}} \frac{r_{0-}\left(2 \Lambda_{4 D} r_{0-}^{2}-1\right)}{\left(r_{0}-r_{0-}\right)\left(r_{0-}-r_{0--}\right)\left(r_{0-}-r_{0 n}\right)}, \\
c_{0--}=-\frac{2}{\Lambda_{4 D}} \frac{r_{0--}\left(2 \Lambda_{4 D} r_{0--}^{2}-1\right)}{\left(r_{0}-r_{0--}\right)\left(r_{0-}-r_{0--}\right)\left(r_{0--}-r_{0 n}\right)}, \\
c_{0 n}=-\frac{2}{\Lambda_{4 D}} \frac{r_{0 n}\left(2 \Lambda_{4 D} r_{0 n}^{2}-1\right)}{\left(r_{0--}-r_{0 n}\right)\left(r_{0}-r_{0 n}\right)\left(r_{0-}-r_{0 n}\right)} .
\end{gathered}
$$

Portanto, as expressões finais para $A(r)$ e $B(r)$, o conjunto completo das soluções construídas, foram escritas como:

$$
A(r)=A_{0}(r)=\frac{\Lambda_{4 D}}{3 r^{2}}\left(r_{c}-r\right)\left(r-r_{+}\right)\left(r-r_{-}\right)\left(r-r_{n}\right)
$$

$\mathrm{e}$

$$
B(r)=A_{0}(r)\left[1+\frac{C}{\left(r-r_{0}\right)^{c_{0}}} \frac{\left(r-r_{0--}\right)^{c_{0--}}}{\left(r-r_{0-}\right)^{c_{0-}}\left(r-r_{0 n}\right)^{c_{0 n}}}\right] .
$$

Como é claro, a função $B(r)$ diverge quando $r \rightarrow r_{0}$. Como entre $r_{+}$e $r_{c}$, ou seja, $r_{+}<$ $r<r_{c}$, temos uma candidata para a região estática do espaço-tempo ${ }^{17}$ e $r_{+}<r_{0}<r_{c}$, o

\footnotetext{
${ }^{17}$ Aqui, por enquanto, $r_{+}$e $r_{c}$ são candidatos a horizontes de eventos e cosmológico, respectivamente.
} 
trabalho de Bronnikov et al. [28] considerou que soluções regulares de (3.45), com constante cosmológica positiva, não existem além da solução de Sitter. Entretanto, de acordo com o nosso trabalho [30], isso mostrou-se equivocado depois de um estudo dos tipos de soluções que surgem a partir de diferentes escolhas do parâmetro $C$ da equação (3.55). Tal parâmetro mostrou-nos três possibilidades: quando $C<0$, temos uma singularidade nua; quando $C=0$, a geometria Reissner-Nordström-de Sitter surge; finalmente, a possibilidade explorada aqui, quando $C>0$ buracos de minhoca assintoticamente de Sitter são gerados.

\section{Singularidade nua}

Quando $C<0$, as funções $A(r)$ e $B(r)$ são positivas para $r_{+}<r<r_{c}$. Entretanto, $r_{+}<r_{0}<r_{c}$, o que implica na divergência de $B(r)$ nesse intervalo (em $r_{0}$, como fica claro em (3.55)). A geometria é estática e bem comportada somente para $r>r_{0}$. Em $r_{0}$ o escalar de Kretschmann diverge, mostrando-nos onde se encontra a singularidade física. Isto é,

$$
\lim _{r \rightarrow r_{0}}\left|R_{\alpha \beta \gamma \delta} R^{\alpha \beta \gamma \delta}\right| \rightarrow \infty .
$$

Como $r_{+}<r_{0}$, temos aqui um cenário que envolve uma singularidade nua, desprotegida de um horizonte de eventos. Este caso não tratamos em nosso trabalho.

\section{Solução de Reissner-Nordström-de Sitter}

Quando $C=0$, a solução obtida é a Reissner-Nordström-de Sitter, como (3.16) e (3.46) indicam. Nessa, a região regular, bem comportada, é $r_{+}<r<r_{c}$. No chamado caso não extremo dessa solução, as superfícies $r=r_{+}$e $r=r_{c}$ indicam o horizonte de eventos e o horizontes cosmológico, respectivamente.

\section{Buracos de minhoca assintoticamente de Sitter}

Por fim, quando $C>0$, temos uma surpreendente geometria que descreve um buraco de minhoca transitável, ou seja, sem horizonte de eventos. Neste caso, a função $B(r)$ não é positiva definida entre $r_{+} \mathrm{e} r_{c}$. Existe um zero a mais para $B(r)$. Tal zero, $r=r_{t h r}$, é de muita importância porque

$$
r_{+}<r_{0}<r_{t h r}<r_{c}
$$

As funções $A(r)$ e $B(r)$, no intervalo $r_{t h r}<r<r_{c}$, são sempre positivas. Portanto, as coordenadas $(t, r, \theta, \phi)$ são válidas na região $r_{t h r}<r<r_{c}$. A extensão analítica da solução, somente para $C>0$, foi feita com o uso do comprimento próprio, definido em (3.44). Com a escolha de uma apropriada constante de integração na definição de $\ell(r)$, a região $r_{t h r}<r<r_{c}$ foi 
mapeada em $0<\ell<\ell_{\max }$, para um valor finito de $\ell_{\max }$. A extensão foi feita com a continuação no intervalo $-\ell_{\max }<\ell<\ell_{\max }$. Como resultado desse procedimento, tivemos uma geometria que descreve um buraco de minhoca com uma garganta em $r=r_{t h r}$. Para realizar a extensão além de $r=r_{c}$, usamos as chamadas coordenadas avançadas $(w, t, \theta, \phi)$ e retardadas $(v, t, \theta, \phi)$ de Eddington, onde $w$ e $v$ são as conhecidas variáveis do cone de luz já definidas em (3.35). Na máxima extensão, a superfície $\ell=\ell_{\max }\left(r=r_{c}\right)$ é um horizonte de Killing, interpretado como um horizonte cosmológico, como pode ser visto no diagrama de Penrose correspondente na figura 3.5. Sistemas de coordenadas que se utilizam da coordenada tartaruga, $r^{*}$, definida em (3.34), ou das coordenadas avançadas ou retardadas, podem então ser usados para cobrir toda a região estática. Nesse caso, $\left.\left\{(t, \ell, \theta, \phi),-\ell_{\max }<\ell<\ell_{\max }\right)\right\}$ é mapeado em $\left\{\left(t, r^{*}, \theta, \phi\right),-\infty<r^{*}<\infty\right\}$ ou $\{(w, v, \theta, \phi),-\infty<w<\infty,-\infty<v<\infty\}$.

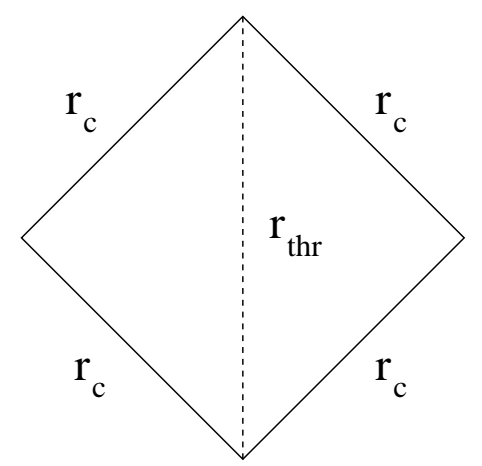

Figura 3.5: Diagrama conforme ou diagrama de Penrose para o caso $C>0$. Temos, aqui, um buraco de minhoca dentro de um horizonte cosmológico $r_{c}$. A linha pontilhada indica sua garganta.

Temos, então, como uma possível interpretação física para essa solução, um buraco de minhoca transitável com simetria esférica num universo em expansão. Para que esta última afirmação fique mais clara, usamos uma forma simplificada de (3.55), com $M=Q=0$, que ainda apreende todas as principais características da solução, para mostrar que temos neste contexto uma densidade de energia negativa, que viola condições de energia, permeando a geometria em foco. Com essa simplificação, as funções da métrica $A(r)$ e $B(r)$ tornaram-se

$$
A(r)=1-\frac{r^{2}}{r_{c}^{2}}
$$

$\mathrm{e}$

$$
B(r)=\left(1-\frac{r^{2}}{r_{c}^{2}}\right)\left[1-C \frac{1}{r\left(r^{2}-r_{0}^{2}\right)^{3 / 2}}\right] .
$$

As expressões para $r_{c}$ e $r_{0}$ também ficaram menores: 


$$
r_{c}=\sqrt{\frac{3}{\Lambda_{4 D}}}, \quad r_{0}=\sqrt{\frac{2}{\Lambda_{4 D}}} .
$$

Igualmente, as demais constantes apresentaram-se como: $r_{n}=r_{0 n}=-r_{0}, c_{0}=c_{0 n}=3 / 2$, $c_{0-}=1$. As constantes que restaram, $r_{-}, r_{--}, r_{0-}, r_{0--}, c_{0--}$, foram anuladas. Assim, as componentes do tensor energia momento efetivo na brana, definidos em (3.29), neste caso simplificado, resumem-se às

$$
\begin{aligned}
8 \pi \rho^{e f} & =\frac{C}{3 r_{0}^{2}} \frac{2 r^{2}-5 r_{0}^{2}}{r\left(r^{2}-r_{0}^{2}\right)^{5 / 2}}, \\
8 \pi p_{\text {rad }}^{e f} & =\frac{C}{r_{0}^{2}} \frac{2 r^{2}-r_{0}^{2}}{r^{3}\left(r^{2}-r_{0}^{2}\right)^{3 / 2}}, \\
8 \pi p_{\text {tan }}^{e f} & =-\frac{C}{6 r_{0}^{2}} \frac{4 r^{4}-\left(2 r_{0} r\right)^{2}+3 r_{0}^{4}}{r^{3}\left(r^{2}-r_{0}^{2}\right)^{5 / 2}} .
\end{aligned}
$$

Podemos ver nas equações (3.61)-(3.63) que neste caso, $C>0$, a densidade de energia e as pressões efetivas não são sempre positivas definidas em $r>r_{0}$, não satisfazem sempre as famosas condições de energia. Como mostra Visser ${ }^{18}$, a matéria próxima à garganta do buraco de minhoca, $r=r_{t h r}$, viola a condição de energia nula. Entretanto, buracos de minhoca transitáveis violam, além dessa, as condições de energia fraca, forte e dominante. O autor, em Lorentzian wormholes: from Einstein to Hawking, elenca pelo menos sete condições de energia. Quando o tensor energia-momento é do tipo I, ou seja, quando pode ser escrito numa base ortonormal para expressar-se como (3.29) - com todas as componentes fora da diagonal principal nulas -, as quatro condições possíveis de serem violadas por buracos de minhoca são (o índice $j$ indica a componente da pressão: para $j=1$, pressão radial, para $j=2$ e 3 , pressão tangencial):

condição nula: $\forall j, \rho+p_{j} \geq 0$;

condição fraca: $\rho \geq 0 \mathrm{e} \forall j, \rho+p_{j} \geq 0$;

condição forte: $\forall j, \rho+p_{j} \geq 0$ e $\rho+\sum_{j} p_{j} \geq 0$;

condição dominante: $\rho \geq 0 \mathrm{e} \forall j, p_{j} \in[-\rho, \rho]$.

Tudo isso corrobora a interpretação de (3.58)-(3.59) como uma geometria com simetria esférica que, para um dado conjunto de parâmetros, descreve um buraco de minhoca num espaço-tempo em expansão, pois a energia escura - aquilo que é tido, nos dias de hoje, como a possível fonte da expansão acelerada do nosso universo - de forma semelhante não satisfaz todas as condições de energia. Dentro do contexto da relatividade geral, buracos de minhocas somente são possíveis a partir da existência de um tipo de matéria exótica que vi-

\footnotetext{
${ }^{18} \mathrm{Cf}$. [73], cap. 12, que é dedicado inteiramente às condições de energia.
} 
ola as condições de energia (a energia escura enquadra-se nesse tipo de matéria). Por outro lado, no contexto de mundos brana que trabalhamos, a existência de buracos de minhoca transitáveis exclui a necessidade de tal matéria exótica. A condição de possibilidade de existência desses interessantes objetos é dada, "apenas", pela influência do bulk na brana - uma influência geométrica.

\subsection{Geodésicas}

Nesta seção, geodésicas foram estudadas na geometria de Sitter exposta anteriormente. Para obtê-las, construímos as três grandezas conservadas relativas à geometria em questão. De acordo com o apêndice $\mathrm{B}$, um espaço-tempo com simetria esférica tem três campos de Killing do tipo espaço associados a essa simetria. Como nesta seção abordamos somente geodésicas equatoriais $(\theta=\pi / 2)$, resta, devido à última assunção, somente o campo do tipo espaço

$$
\xi_{\phi}=\frac{\partial}{\partial \phi}
$$

que em coordenadas é escrito como

$$
\xi_{\phi}^{\mu}=(0,0,0,1), \quad \xi_{\phi \mu}=\left(0,0,0, r^{2}\right) .
$$

Devido ao fato de ser estacionária, a geometria que descreve um buraco de minhoca da seção anterior tem o campo de Killing do tipo tempo

$$
\xi_{t}=\frac{\partial}{\partial t},
$$

que em coordenadas

$$
\xi_{t}^{\mu}=(1,0,0,0), \quad \xi_{t \mu}=(-1,0,0,0) .
$$

Tais campos forneceram-nos as equações das geodésicas desejadas. Como consta no apêndice B, campos de Killing dizem-nos sobre quantidades conservadas.

A equação de movimento de uma partícula num espaço-tempo, sujeita somente ao campo gravitacional, escrita na base das coordenadas, é

$$
\frac{d^{2} x^{\mu}}{d \lambda^{2}}+\Gamma_{v \gamma}^{\mu} \frac{d x^{\nu}}{d \lambda} \frac{d x^{\gamma}}{d \lambda}=0
$$

onde $\lambda$ é um parâmetro afim importante, $\mathrm{e}$ 


$$
\Gamma_{v \gamma}^{\mu}=\frac{1}{2} g^{\mu \rho}\left(\partial_{v} g_{\gamma \rho}+\partial_{\gamma} g_{v \rho}-\partial_{\rho} g_{v \gamma}\right)
$$

é a conexão afim ou símbolo de Christoffel, com $\partial_{\mu}$ representando o operador derivada parcial numa métrica $g_{\mu \nu}$. Uma trajetória dada pela equação (3.68) é chamada de geodésica; nela, o seu vetor tangente - representado em suas componentes por $d x^{\mu} / d \lambda$ - é transportado paralelamente a si mesmo. Além disso, na parametrização escolhida em (3.68), a norma desse vetor tangente permanece inalterada. Sem correr o risco de perder generalidade, geodésicas equatoriais podem ser escritas como

$$
x^{\mu}(\lambda)=(t(\lambda), r(\lambda), \theta(\lambda), \phi(\lambda))=\left(t(\lambda), r(\lambda), \frac{\pi}{2}, \phi(\lambda)\right) .
$$

Associadas aos campos $\xi_{t}$ e $\xi_{\phi}$ temos as quantidades conservadas $E$ e $L$ (energia e momento angular), numa geodésica, definidas pelas equações

$$
E=-\xi_{t \mu} \frac{d x^{\mu}(\lambda)}{d \lambda}=+A(r(\lambda)) \frac{d t(\lambda)}{d \lambda}
$$

$\mathrm{e}$

$$
L=\xi_{\phi \mu} \frac{d x^{\mu}(\lambda)}{d \lambda}=+r(\lambda)^{2} \frac{d \phi(\lambda)}{d \lambda} .
$$

Como na relatividade geral a conexão é compatível com a métrica e, portanto, o tensor métrico é transportado paralelamente ao longo da geodésica, temos, a partir disso, mais uma quantidade conservada, a constante $\mathscr{K}$

$$
-2 \mathscr{K}=g_{\mu v} \frac{d x^{\mu}(\lambda)}{d \lambda} \frac{d x^{v}(\lambda)}{d \lambda}=-A(r(\lambda))\left(\frac{d t(\lambda)}{d \lambda}\right)^{2}+\frac{1}{B(r(\lambda))}\left(\frac{d r(\lambda)}{d \lambda}\right)^{2}+r(\lambda)^{2}\left(\frac{d \phi(\lambda)}{d \lambda}\right)^{2} .
$$

Com as três quantidade conservadas, $E, L, \mathscr{K}$, o problema da determinação das geodésicas em espaços-tempo com simetria esférica pôde ser colocado na seguinte forma, em termos de quatro equações:

$$
\begin{aligned}
\dot{t} & =\frac{d t(\lambda)}{d \lambda}=f_{t}(r) \\
\dot{r}^{2} & =\left(\frac{d r(\lambda)}{d \lambda}\right)^{2}=f_{r}(r) \\
\theta & =\frac{\pi}{2} \\
\dot{\phi} & =\frac{d \phi(\lambda)}{d \lambda}=f_{\phi}(r)
\end{aligned}
$$


com as funções $f_{t}, f_{r}$ e $f_{\phi}$ definidas como

$$
\begin{aligned}
f_{t}(r) & =\frac{E}{A(r)}, \\
f_{r}(r) & =\frac{B(r)}{A(r)} E^{2}-B(r) \frac{L^{2}}{r^{2}}-2 \mathscr{K} B(r), \\
f_{\phi}(r) & =\frac{L}{r^{2}} .
\end{aligned}
$$

O problema, então, está desacoplado. Ou seja, a equação (3.75) é independente das demais — uma vez resolvida e a solução $r(\lambda)$ determinada, as equações (3.74) e (3.77) têm suas soluções independentes.

Para o caso exposto na seção anterior - um buraco de minhoca numa brana assintoticamente de Sitter -, a equação (3.75) mostrou-nos características notáveis em dois pontos: $r=r_{t h r} \mathrm{e} r=r_{c}$, isto é, na garganta e no horizonte cosmológico. No primeiro, um ponto de inflexão surgiu, segundo o qual $\dot{r}=0$. No segundo, a função $\dot{r}$ divergiu. Contudo, como vimos na seção anterior, o sistema de coordenadas $(t, r, \theta, \phi)$ só é valido no intervalo $r_{t h r}<r<r_{c}$. Então, tais características notáveis devem-se ao inadequado sistema de coordenadas.

Como foi dito, um sistema de coordenadas mais adequado para esse problema é aquele que se utiliza da coordenada tartaruga $r^{*}$, definida em (3.34). Com as coordenadas $\left(t, r^{*}, \theta, \phi\right)$, a equação (3.75) tornou-se

$$
\left(\dot{r}^{*}\right)^{2}=\left(\frac{d r^{\star}(\lambda)}{d \lambda}\right)^{2}=g_{r}(r)
$$

sendo

$$
g_{r}(r)=\frac{1}{A(r)}\left[\frac{E^{2}}{A(r)}-\frac{L^{2}}{r^{2}}-2 \mathscr{K}\right]
$$

pois

$$
\frac{d r}{d \lambda}=\frac{d r}{d r^{\star}} \frac{d r^{\star}}{d \lambda}=\sqrt{A(r) B(r)} \frac{d r^{\star}}{d \lambda} .
$$

Dessa forma, como veremos, tornou-se claro que a garganta, $r^{*}=0$, não é, em todos os casos, um ponto de inflexão. Vejamos dois tipos de trajetórias: radiais e não radiais.

\subsubsection{Trajetórias radiais}

As trajetórias radiais são aqui geodésicas tais que

$$
\dot{\phi}=0 \text {. }
$$


De tal forma, a órbita — projeção da geodésica numa hipersuperfície onde $t$ é constante aponta na direção radial. Com a condição (3.84) para um certo valor do parâmetro afim, $\lambda=\lambda_{0}$, a equação (3.77) ficou

$$
\frac{L}{r\left(\lambda_{0}\right)^{2}}=0
$$

ou seja, em $\lambda=\lambda_{0}$, a constante $L$ é nula. Mas como já dissemos, $L$ é uma das três constantes de movimento. Portanto, $L$ é nula para qualquer $\lambda$, em qualquer ponto da geodésica radial, de acordo com (3.84). Com a condição $L=0$, (3.82) simplificou-se

$$
g_{r}(r)=\frac{1}{A(r)}\left[\frac{E^{2}}{A(r)}-2 \mathscr{K}\right] .
$$

\section{Geodésicas radiais tipo luz}

Para o caso de uma trajetória radial, cujo vetor tangente é do tipo luz, $\mathscr{K}=0$, com isso

$$
g_{r}(r)=\frac{E^{2}}{A(r)^{2}}
$$

portanto,

$$
\frac{d r^{\star}}{d \lambda}>0
$$

Ou seja, não existem pontos de inflexão para geodésicas radiais do tipo luz, pois $A(r)$ e $d r^{*} / d \lambda$ são funções positivas definidas na região de interesse, entre a garganta e o horizonte cosmológico. Uma interpretação para este último resultado é a seguinte: feixes de luz radiais sempre atravessam a garganta, não importando quão energéticos são.

\section{Geodésicas radiais do tipo tempo}

Para geodésicas radiais do tipo tempo, temos $\mathscr{K}>0$, isto é, a função $g_{r}(r)$ pode ter um zero real na região que nos interessa. Dessa forma, um ponto de inflexão é possível para esse tipo de curva, conforme a figura 3.6. Se supusermos geodésicas radiais que partem (num certo $\lambda=\lambda_{0}$ ) do lado direito do diagrama de Penrose, mostrado na figura 3.5, na direção da garganta

$$
r^{*}\left(\lambda_{0}\right)>0 \quad \text { e } \frac{d r^{\star}\left(\lambda_{0}\right)}{d \lambda}<0
$$

Se, além disso, impusermos que o ponto de inflexão $r^{*}=r_{i n f}^{*}$ seja maior do que zero, ou seja, esteja também no mesmo lado do diagrama de Penrose tal que $0<r_{\text {inf }}^{*}<r^{*}\left(\lambda_{0}\right)$, e que $r_{\text {inf }}$ seja o valor da coordenada $r$ de modo que $r^{*}=r_{i n f}^{*}$, então, a energia associada à geodésica, 
segundo (3.81) e (3.86), será

$$
\frac{E^{2}}{A\left(r_{\text {inf }}\right)}-2 \mathscr{K}=0
$$

ou seja,

$$
E=\sqrt{2 \mathscr{K} A\left(r_{\text {inf }}\right)} .
$$

Mas a função $A(r)$ é decrescente, com máximo valor em $r=r_{t h r}$, no intervalo $r_{t h r}<r<r_{c}$. Portanto, existe um valor máximo de $E_{\max }$ segundo o qual um ponto de inflexão está presente. Quando indicamos esse máximo, considerando que a inflexão ocorre na garganta, $r=r_{t h r}$, obtivemos

$$
E_{\text {max }}=\sqrt{2 \mathscr{K} A\left(r_{t h r}\right)}=\sqrt{2 \mathscr{K}\left(1-\frac{2 M}{r_{t h r}}+\frac{Q^{2}}{r_{t h r}^{2}}-\frac{\Lambda}{3} r_{t h r}^{2}\right)} .
$$

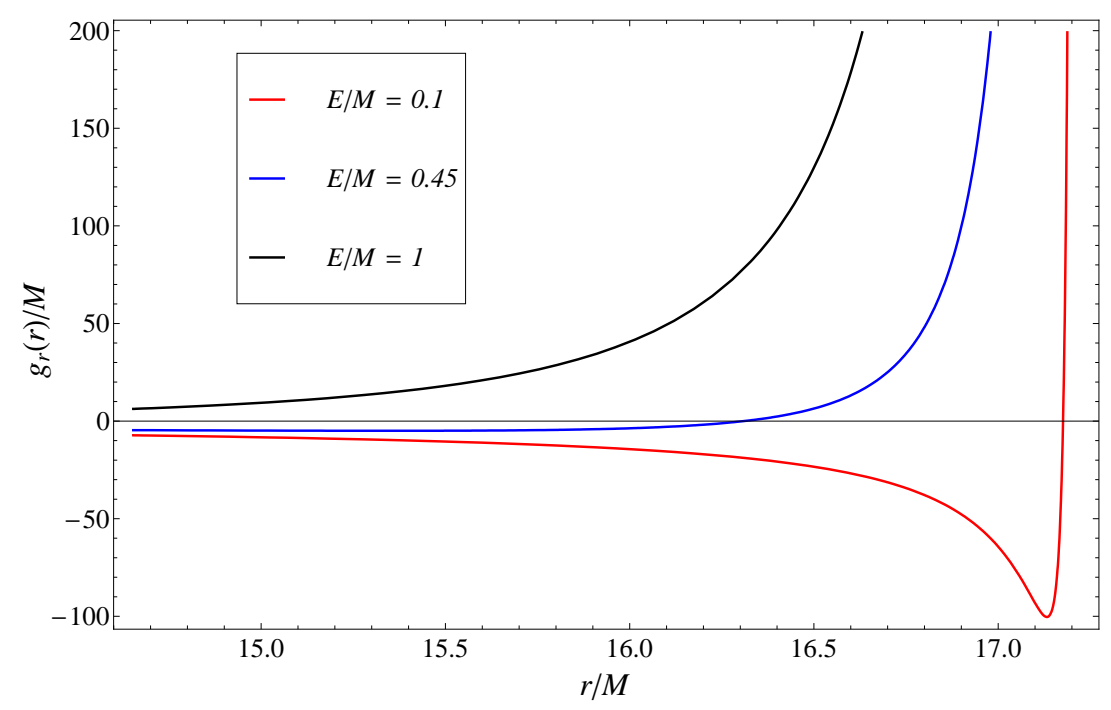

Figura 3.6: Função $g_{r}(r)$ para alguns valores de $E \operatorname{com} L=0$ (trajetórias radiais), dentro do intervalo $r_{t h r}<r<r_{c}$. Neste gráfico $E_{\max } / M$ vale 0.74 . Com isso, constata-se que quando $E<E_{\max }$ há um ponto de inflexão, e a partícula, que percorre uma geodésica do tipo tempo, sofre um ricochete.

Para valores de $E$ maiores do que $E_{\max }$ não há ponto de inflexão. Uma interpretação para esse resultado é a seguinte: partículas massivas com energias menores do que $E_{\max }$ - descrevendo geodésicas radiais inicialmente na direção da garganta do buraco de minhoca sofrem um ricochete e retornam na direção do horizonte cosmológico. Quando uma partícula tem energia igual à energia máxima $E_{\max }$, sofre um ricochete na garganta do buraco de minhoca, ou seja, o ponto de inflexão é $r_{\text {inf }}=r_{t h r}$. Por fim, partículas energéticas com $E>E_{\max }$ não sofrem qualquer ricochete - atravessam a garganta da mesma forma que raios de luz. 

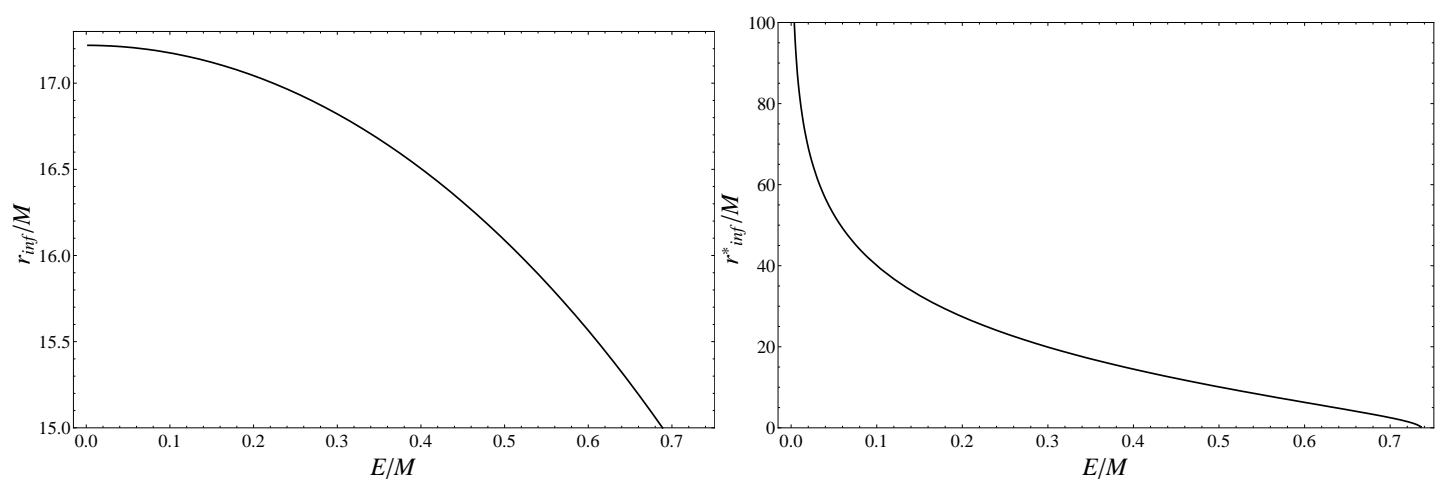

Figura 3.7: Pontos de inflexão em função do parâmetro $E / M$ para o caso de trajetórias radiais. À esquerda a coordenada $r_{\text {inf }}$; à direita a coordenada tartaruga $r_{\text {inf }}^{*}$.

\subsubsection{Trajetórias não radiais}

Neste caso, $d \phi\left(\lambda_{0}\right) / d \lambda \neq 0$ em algum ponto da trajetória. Com isso, $L \neq 0$. Portanto, mesmo em geodésicas do tipo luz podemos ter pontos de inflexão e ricochetes. Para um dado ponto de inflexão, $r=r_{\text {inf }}$, em geodésicas do tipo tempo ou nula, temos

$$
\frac{E^{2}}{A\left(r_{i n f}\right)}-\frac{L^{2}}{r_{i n f}^{2}}-2 \mathscr{K}=0,
$$

de tal forma que

$$
E=\sqrt{A\left(r_{\text {inf }}\right)\left(\frac{L^{2}}{r_{\text {inf }}^{2}}+2 \mathscr{K}\right)} .
$$

O comportamento qualitativo das geodésicas não radiais, do tipo tempo ou luz, é o mesmo apresentado para as geodésicas do tipo tempo radiais. Porém, fisicamente, o termo centrípeto na equação radial pode forçar a existência de um ponto de inflexão. Como já dissemos, mesmo para luz pode ocorrer um ricochete, mas neste caso, o raio de luz é curvado pelo buraco de minhoca, sem nele entrar. 



\section{Capítulo 4}

\section{Simetria axial em mundos brana}

Este capítulo aborda soluções estacionárias com simetria axial das equações do campo gravitacional num contexto de mundos brana. Mas para começá-lo, apresentamos a solução, no contexto da teoria da relatividade geral, mais famosa que apresenta esse tipo de simetria a solução de Kerr. Esta, um grande êxito da relatividade geral obtido tardiamente ${ }^{1}$, representa um buraco negro com rotação. Sua presença neste capítulo - um capítulo dedicado ao contexto de mundos brana — faz-se necessária. Usamo-la como referência, como algo para comparar com as soluções criadas num contexto diferente da relatividade geral. Em seguida, comentamos a sua generalização para os casos anti-de Sitter (AdS) e de Sitter (dS). Num contexto de mundos brana, apresentamos a solução com simetria axial sem constante cosmológica obtida por Aliev e Gümrükçüoglu no trabalho [33]. Neste mesmo contexto, trazemos as soluções obtidas por nós em Rotating black holes in a Randall-Sundrum brane with a cosmological constant [35], que generalizam os resultados dos dois autores citados. Tais soluções apresentam um ingrediente a mais: a constante cosmológica. Por fim, na seção 4.5, tratamos das equações das geodésicas das novas métricas construídas. Estudos sobre geodésicas são importantes pois mostram a razoabilidade de espaços-tempo que podem ser considerados fisicamente reais.

\subsection{Soluções de Kerr e Kerr-(anti)-de Sitter}

No contexto da teoria da relatividade geral, a solução de Kerr, obtida em 1963 por Roy Patrick Kerr [4], nas coordenadas de Boyer-Lindquist $(t, r, \theta, \phi)$ apresenta dois campos de

\footnotetext{
${ }^{1}$ A solução de Kerr, que consta pela primeira vez no trabalho Gravitational field of a spinning mass as an example of algebraically special metrics de Roy P. Kerr em 1963 [4], surgiu quase cinquenta anos depois da solução de Schwarzschild.
} 
Killing devido à sua independência em relação às coordenadas $t$ e $\phi$. Ou seja, a métrica

$$
\begin{aligned}
d s^{2} & =-\frac{1}{\Sigma}\left(\Delta_{r}-a^{2} \operatorname{sen}^{2} \theta\right) d t^{2}-\frac{2 a}{\Sigma}\left[\left(r^{2}+a^{2}\right)-\Delta_{r}\right] \operatorname{sen}^{2} \theta d t d \phi+ \\
& +\frac{\Sigma}{\Delta_{r}} d r^{2}+\Sigma d \theta^{2}+\frac{1}{\Sigma}\left[\left(r^{2}+a^{2}\right)^{2}-a^{2} \Delta_{r} \operatorname{sen}^{2} \theta\right] \operatorname{sen}^{2} \theta d \phi^{2}
\end{aligned}
$$

com

$$
\Delta_{r}=r^{2}+a^{2}-2 M r \quad \text { e } \quad \Sigma=r^{2}+a^{2} \cos ^{2} \theta,
$$

não depende dessas duas coordenadas, como fica claro acima. Então, de acordo com o apêndice $\mathrm{B}^{2}$, podemos construir dois campos de Killing na base das coordenadas usadas, de tal forma que

$$
\xi_{t}=\frac{\partial}{\partial t} \quad \text { e } \quad \xi_{\phi}=\frac{\partial}{\partial \phi} .
$$

Além disso, como pode ser visto no apêndice citado, por (4.1) ser independente de $t$, segue que a métrica de Kerr é estacionária. A simetria axial surge por possuir também um campo de Killing $\xi_{\phi}$ que conduz à isometria cujas órbitas são curvas do tipo espaço fechadas. Então, a métrica de Kerr pode ser expressa na forma mais geral que uma métrica estacionária com simetria axial pode ser escrita. Tal forma é indicada pela equação (B.11). A solução (4.1) tem dois parâmetros, $a$ e $M$. O primeiro reduz a métrica de Kerr à de Schwarzschild desde que seja nulo; é chamado de parâmetro de rotação. $\mathrm{O}$ segundo, $M$, é identificado com a massa do buraco negro ${ }^{3}$.

A métrica de Kerr nas coordenadas $(t, r, \theta, \phi)$ mostra-se problemática em $\Delta_{r}=0$ ou $\Sigma=0$. Quando uma destas duas condições é satisfeita, a métrica (4.1) diverge. Com o escalar de Kretschmann, $R_{\alpha \beta \gamma \delta} R^{\alpha \beta \gamma \delta}$, mostra-se que a métrica de Kerr tem somente uma singularidade física ou intrínseca quando $\Sigma=r^{2}+a^{2} \cos ^{2} \theta$ for nulo e $M \neq 0$. Ou seja,

$$
R_{\alpha \beta \gamma \delta} R^{\alpha \beta \gamma \delta} \propto \frac{1}{\Sigma^{6}} .
$$

Então, quando $r=\cos \theta=0$ esse escalar diverge. Segundo Hawking e Ellis [14], no capítulo dedicado a soluções exatas das equações de Einstein, pode-se mostrar que a singularidade física na métrica de Kerr tem formato anelar. Com uma mudança de coordenadas, tal que o espaço-tempo seja descrito por $(\bar{t}, x, y, z)$, o cálculo do escalar de Kretschmann indica

\footnotetext{
${ }^{2}$ No apêndice $B$, apresentamos os conceitos de simetrias esférica e axial, utilizando campos de Killing. Além disso, enfatizamos a importância destes para quantidades conservadas numa variedade $\mathscr{M}$ equipada com uma métrica lorentziana.

${ }^{3}$ Discute-se, na seção 4.5 desta tese, que em geometrias assintoticamente planas, a massa de um buraco negro pode ser determinada por meio das integrais de Komar. Com estas, $M$ da solução de Kerr, por exemplo, pode ser interpretado como massa. Entretanto, em geometrias assintoticamente não planas, a questão é outra. Nestas, a integral de Komar diverge. Na seção 4.5, algumas opções para resolver este problema foram expostas.
} 
que a singularidade situa-se em $z=0(\theta=\pi / 2$ nas coordenadas de Boyer-Lindquist), com $x^{2}+y^{2}=a^{2}$, exibindo, então, o formato de um anel com raio $a$. Por outro lado, a condição $\Delta_{r}=0$ conduz a uma singularidade não intrínseca à métrica. Tal é causada por um sistema de coordenadas inadequado à região da variedade em foco. Como o conhecido $r=2 M$ da métrica de Schwarzschild (um zero da equação $g^{r r}=1-2 M / r=0$ ), os zeros de $g^{r r}$ da solução de Kerr, ou seja, os zeros de $\Delta_{r}=0$, fornecem os raios dos horizontes, que são superfícies cujos vetores tangentes são do tipo nulo ${ }^{4}$. Na métrica de Kerr, então, esses zeros são o horizonte interno, $r_{-}$, e o horizonte externo (o horizonte de eventos), $r_{+}$; cujas expressões são:

$$
r_{ \pm}=M \pm \sqrt{M^{2}-a^{2}} .
$$

Vale a pena enfatizar que as expressões para os horizontes somente existem quando $a^{2} \leq M^{2}$. Quando esta relação não é obedecida, $\Delta_{r}$ não tem raízes reais, e a solução de Kerr fornece uma singularidade nua - uma singularidade sem horizonte de eventos. Para as métricas com simetria axial construídas por nós, discutidas nas seções 4.3 e 4.4, levaremos em conta somente a condição de que o parâmetro de rotação $a$ seja menor ou igual ao parâmetro $M$. Quando $a=M$, temos o chamado caso extremo; neste, $r_{-}=r_{+}$.

O problema na métrica de Kerr, cuja origem remonta a um sistema de coordenadas inaplicável para $r \leqslant r_{+}$, pode ser resolvido com uma adequada mudança de coordenadas $(t, r, \theta, \phi) \rightarrow(u, r, \theta, \varphi)$. Isto é, com

$$
d u=d t+\left(\frac{r^{2}+a^{2}}{\Delta_{r}}\right) d r \quad \text { e } \quad d \varphi=d \phi+\frac{a}{\Delta_{r}} d r
$$

a métrica (4.1) torna-se

$$
\begin{aligned}
d s^{2} & =-\frac{1}{\Sigma}\left(\Delta_{r}-a^{2} \operatorname{sen}^{2} \theta\right) d u^{2}-\frac{4 a m r}{\Sigma} \operatorname{sen}^{2} \theta d u d \varphi+2 d r d \varphi-2 a \operatorname{sen}^{2} \theta d r d \varphi \\
& +\Sigma d \theta^{2}+\frac{1}{\Sigma}\left[\left(r^{2}+a^{2}\right)^{2}-a^{2} \Delta_{r} \operatorname{sen}^{2} \theta\right] \operatorname{sen}^{2} \theta d \varphi^{2},
\end{aligned}
$$

analítica em $r=r_{+}$e $r=r_{-}$. De forma semelhante, pode-se fazer a extensão da métrica utilizando $(t, r, \theta, \phi) \rightarrow(v, r, \theta, \varsigma)$, onde

$$
d v=d t-\left(\frac{r^{2}+a^{2}}{\Delta_{r}}\right) d r \quad \text { e } \quad d \varsigma=d \phi-\frac{a}{\Delta_{r}} d r .
$$

Igualmente, nesse caso, temos (4.7), mas com $-v \mathrm{e}-\varsigma$ nos lugares de $u$ e $\varphi$, respectivamente. A chamada máxima extensão ocorre com a combinação dessas duas extensões, com toda a

\footnotetext{
${ }^{4}$ No apêndice $\mathrm{C}$ há uma pequena discussão sobre a natureza dos horizontes.
} 
variedade $\mathscr{M}$ coberta por um atlas analítico, que é uma coleção de sistemas de coordenadas.

A solução de Kerr possui uma interessante e mais rica estrutura em relação à solução de Schwarzschild. Esta estrutura exibe cinco regiões identificáveis no espaço-tempo. Para indicá-las, vamos, primeiro, obter a norma do campo de Killing $\xi_{t}$. Na base das coordenadas, $\xi_{t}$ tem as seguintes componentes: $(1,0,0,0)$; sua norma é dada por $\xi_{t}^{2}=g_{\mu \nu} \xi_{t}^{\mu} \xi_{t}^{\nu}$. Isso nos leva ao resultado

$$
\xi_{t}^{2}=g_{t t}=-\frac{1}{\Sigma}\left(\Delta_{r}-\Delta_{\theta} a^{2} \operatorname{sen}^{2} \theta\right) .
$$

O campo $\xi_{t}$ torna-se nulo em duas superfícies, onde $\xi_{t}^{2}=0$. Tais são chamadas superfícies de Killing provenientes de $\xi_{t}$. A superfície interna indicamos por $S_{-}$, e a externa é indicada por $S_{+}$. Uma parte da região entre elas é de muito interesse. Mas antes de anunciar o nome desta interessante região, onde vetores de Killing $\xi_{t}$ são tipo espaço, convém relacionar essas superfícies com os horizontes interno e de eventos (o mais externo). As cinco regiões acima mencionadas, de acordo com a distância em relação à origem do sistema de coordenadas, são: $0<S_{-}<r_{-}<r_{+}<S_{+}<\infty$. Entre $r_{+}$e $S_{+}$temos a chamada ergosfera, onde o campo $\xi_{t}$ assume uma norma que o torna do tipo espaço. A ergosfera é uma região ainda acessível a um observador muito distante. Nela, para percorrer as órbitas do campo $\xi_{t}$, é preciso percorrer curvas do tipo espaço 5 .

A generalização da solução de Kerr, que é assintoticamente plana (isto é, para $r \rightarrow \infty$ a métrica de Kerr torna-se a métrica de Minkowski em coordenadas polares esféricas), para os casos assintoticamente de Sitter e anti-de Sitter, foi obtida por Carter no seu Hamilton-Jacobi and Schrödinger separable solutions of Einstein's equations [6], em 1968. Nas coordenadas de Boyer-Lindquist $(t, r, \theta, \phi)$, essa generalização é escrita como:

$$
\begin{aligned}
d s^{2} & =-\frac{1}{\Sigma}\left(\Delta_{r}-\Delta_{\theta} a^{2} \operatorname{sen}^{2} \theta\right) d t^{2}-\frac{2 a}{\left(1+\frac{\Lambda}{3} a^{2}\right) \Sigma}\left[\left(r^{2}+a^{2}\right) \Delta_{\theta}-\Delta_{r}\right] \operatorname{sen}^{2} \theta d t d \phi+ \\
& +\frac{\Sigma}{\Delta_{r}} d r^{2}+\frac{\Sigma}{\Delta_{\theta}} d \theta^{2}+\frac{1}{\left(1+\frac{\Lambda}{3} a^{2}\right)^{2} \Sigma}\left[\left(r^{2}+a^{2}\right)^{2} \Delta_{\theta}-\Delta_{r} a^{2} \operatorname{sen}^{2} \theta\right] \operatorname{sen}^{2} \theta d \phi^{2},(4
\end{aligned}
$$

$\operatorname{com} \Sigma$ sendo o mesmo da solução de Kerr. A constante $\Lambda$ representa a constante cosmológica, o termo a mais nas equações de Einstein que possibilita a construção da métrica acima. Os novos termos na métrica são

$$
\Delta_{r}=\left(r^{2}+a^{2}\right)\left(1-\frac{\Lambda}{3} r^{2}\right)-2 M r \text { e } \Delta_{\theta}=1+\frac{\Lambda}{3} a^{2} \cos ^{2} \theta .
$$

As generalizações com carga, cujo comportamento assintótico é plano (Kerr-Newman), de Sitter (Kerr-Newman-dS) e anti-de Sitter (Kerr-Newman-AdS), são as soluções comentadas

\footnotetext{
${ }^{5} \mathrm{Na}$ seção 12.3 de [49], pode-se encontrar uma pequena discussão sobre esse efeito.
} 
e expostas acima com a adição de um campo eletromagnético $\left(F_{\mu \nu} \neq 0\right)$, geralmente o campo elétrico. Diferem das soluções apresentadas porque têm o termo $Q^{2}$ na função $\Delta_{r}$. A presença deste termo muda a estrutura do espaço-tempo, como pode ser visto na seção 12.3 do General relativity, de Wald. Nas próximas três seções, apresentamos métricas estacionárias com simetria axial construídas num outro cenário — os mundos brana. A primeira, assintoticamente plana, foi desenvolvida por Aliev e Gümrükçüoglu, a segunda e a terceira métricas, assintoticamente AdS e dS, foram obtidas por nós em [35].

\subsection{Solução assintoticamente plana}

No trabalho Charged rotating black holes on a 3-brane [33], em 2005, Aliev e Gümrükçüoglu descrevem uma solução estacionária com simetria axial num cenário brana do tipo RandallSundrum. Para obtê-la, usaram as equações do campo gravitacional induzidas na brana derivadas num trabalho por eles mesmos publicado [34]. Levando-se em consideração vácuo no bulk, as equações desses autores coincidem com as equações deduzidas por Shiromizu et al. em [26]. Estas últimas - as mesmas usadas por nós ${ }^{6}$, como será visto nas próximas duas seções. Então, para uma 3-brana sem campos de matéria e sem constante cosmológica, as equações resolvidas por Aliev et al. foram

$$
R_{\mu \nu}=-E_{\mu \nu}
$$

que implicam em

$$
R=0
$$

onde $R_{\mu \nu}$ é o tensor de Ricci, $E_{\mu \nu}$ é um tensor ${ }^{7}$ que tem traço nulo $\left(E_{\mu}^{\mu}=0\right)$, e $R$ é o escalar de Ricci ou de curvatura. Para resolver as equações acima, os autores usaram o ansatz de Kerr-Schild ${ }^{8}$ :

$$
d s^{2}=d s_{\text {plana }}^{2}+H\left(l_{\mu} d x^{\mu}\right)^{2}
$$

sendo $d s_{\text {plana }}^{2}$ a métrica plana ou de Minkowski, $H(r, \theta)$, uma função de $r$ e $\theta$, e $l_{\mu}$ representa um vetor de norma nula. Com esse recurso, (4.14) forneceu a Aliev et al. uma maneira

\footnotetext{
${ }^{6}$ A dedução desse importante resultado, de Shiromizu et al., um trabalho muitíssimo citado, está no apêndice A.

${ }^{7} \mathrm{O}$ significado de $E_{\mu \nu}$ é o mesmo tanto para Aliev. et al. quanto para Shiromizu et al.: $E_{\mu \nu}$ é a chamada parte elétrica do 5-dimensional tensor de Weyl.

8“'O ansatz de Kerr-Schild (...) para a métrica induzida na brana, grosseiramente falando, implica que a métrica exata para um buraco negro na brana pode ser expressa na forma de sua aproximação linear em torno da métrica plana" (cf. [33], p. 4). O ansatz usado por esses autores não possui constante cosmológica e foi construído por Kerr e Schild em 1965, cf. [75].
} 
de escrever uma métrica assintoticamente plana numa 3-brana com a propriedade de possuir simetria axial. Nas coordenadas $(\tau, r, \theta, \phi)$, tal ansatz toma a forma

$$
\begin{aligned}
d s^{2} & =\left[-(d \tau+d r)^{2}+d r^{2}+\Sigma d \theta^{2}+\left(r^{2}+a^{2}\right) \operatorname{sen}^{2} \theta d \phi^{2}+2 \operatorname{asen}^{2} \theta d r d \phi\right] \\
& +H\left(d \tau-\operatorname{asen}^{2} \theta d \phi^{2}\right) .
\end{aligned}
$$

O problema, então, foi determinar a função $H$. Nas coordenadas acima expostas, os autores usaram (4.15) na equação do campo (4.13) - que os conduziu à

$$
\frac{\partial^{2} H}{\partial r^{2}}+\frac{4 r}{\Sigma} \frac{\partial H}{\partial r}+\frac{2}{\Sigma} H=0 .
$$

Ou seja,

$$
H=\frac{2 M r}{\Sigma}-\frac{q}{\Sigma}
$$

onde $\Sigma$ é o mesmo da solução de Kerr. Com a determinação de $H$, a métrica (4.15) pôde ser explicitada. Entretanto, os parâmetros $M$ e $q$ são constantes de integração, e tornaram-se claros seus sentidos físicos quando se passou a métrica (4.15) para as coordenadas de BoyerLindquist com as seguintes transformações:

$$
d \tau=d t-\frac{r^{2}+a^{2}}{\Delta_{r}} d r
$$

e

$$
d \phi=d \varphi-\frac{a}{\Delta_{r}} d r
$$

com

$$
\Delta_{r}=r^{2}+a^{2}-2 M r+q
$$

Com essas transformações, a métrica tornou-se a mesma da equação (4.1), com um acréscimo do parâmetro $q$ na função $\Delta_{r}$. Os autores, então, enfatizaram a semelhança que existe entre a métrica construída por eles e a métrica de Kerr-Newman, no contexto da relatividade geral, que descreve um buraco negro estacionário com simetria axial e carga. Por esse motivo, interpretaram os parâmetros $M$ e $a$ como massa e razão do momento angular pela massa, respectivamente, do buraco negro. Além disso, afirmaram que " a partir da forma assintótica da métrica (...), segue que o parâmetro q é, num certo sentido, uma carga do tipo Coulomb."9 Tal parâmetro mede a influência do bulk na brana. Enfatizamos que a "carga de maré" não corresponde a uma carga elétrica na brana. Nesta, o tensor eletromagnético é nulo $\left(F_{\mu \nu}=0\right)$. Este é o motivo para denominar $q$ como uma carga induzida, um efeito do bulk na brana.

\footnotetext{
${ }^{9}$ Cf. [33], p. 5 (trocamos neste trabalho $\beta$, usada no trabalho citado, por $q$ ).
} 
Os zeros de (4.20) correspondem aos horizontes: um interno e outro externo, o horizonte de eventos. A expressão para este último é simples e pode ser colocada aqui ${ }^{10}$

$$
r_{+}=M+\sqrt{M^{2}-a^{2}-q}
$$

Para ser real, a expressão acima tem que obedecer a $M^{2} \geq a^{2}+q$. A igualdade ocorre no caso extremo, tornando $r_{+}=M$. Neste caso, quando $q$ for negativa, $a$ será maior do que $M$. Então, os autores afirmaram que "o efeito do bulk na brana pode prover um mecanismo para girar um buraco negro estacionário com simetria axial na brana de tal forma que seu parâmetro de rotação exceda sua massa. No entanto, tal mecanismo é impossível na relatividade geral"11, como vimos na seção 4.1, quando a métrica de Kerr foi brevemente discutida. Naquele caso, $a^{2} \leq M^{2}$ para que tenhamos um buraco negro com um horizonte de eventos usual. Aqui, podemos ter o contrário. Outro resultado importante do trabalho de Aliev e Gümrükçüoglu é a possibilidade da "carga de maré" amplificar os efeitos gravitacionais na brana, isto é, a influência do bulk na brana pode tornar o raio do horizonte de eventos maior neste contexto do que no contexto da relatividade geral, pois $q$ também assume valores negativos ${ }^{12}$.

De forma análoga à métrica de Kerr, os zeros de $g_{t t}$ fornecem as superfícies de Killing superfícies onde $\xi_{t}^{2}=0$. A maior de todas, o limite ou o raio da ergosfera, é dada por

$$
S_{+}=M+\sqrt{M^{2}-a^{2} \cos ^{2} \theta-q} .
$$

Tal como o horizonte de eventos, $S_{+}$torna-se maior quando $q<0$. Quando $\theta=0$ ou $\theta=\pi$, $S_{+}$coincide com $r_{+}$. No caso extremo, $M^{2}=a^{2}+q$ e $r_{+}=M$, a ergosfera $\left(r_{+}<r<S_{+}\right)$ torna-se

$$
M<r<M+\operatorname{sen} \theta \sqrt{M^{2}-q}
$$

Pode-se notar que, $\operatorname{com} q<0$, essa importante região é maior no contexto de mundos brana do que na relatividade geral. Dessa forma, Aliev et al. concluem que “(...) buracos negros, em rotação em mundos brana com carga de maré negativa, são objetos mais energéticos no sentido de extração de energia rotacional de suas ergosferas." 13

\footnotetext{
${ }^{10}$ Como veremos nas próximas duas seções, as soluções assintoticamente AdS e dS têm mais do que dois horizontes. Suas expressões correspondentes não serão colocadas no texto devido ao tamanho que possuem.

${ }^{11}$ Ibid., p. 6. Itálico dos autores.

${ }^{12} \mathrm{Na}$ solução Kerr-Newman, $r_{+}=M+\sqrt{M^{2}-a^{2}-Q^{2}}$.

${ }^{13}$ Ibid., p. 6. Itálico dos autores. O mecanismo de extração de energia de rotação de buracos negros é o mecanismo de Penrose, cf. [49], seção 12.4.
} 


\subsection{Solução assintoticamente AdS}

Seguindo a alternativa proposta em [33], construímos em [35] uma métrica estacionária com simetria axial e constante cosmológica negativa num cenário brana do tipo RandallSundrum [17]. As equações do campo gravitacional induzido numa 3-brana por um bulk 5dimensional assintoticamente $\mathrm{AdS}$, para um bulk e uma brana com tensores energia-momento nulos, reduzem-se, de acordo com (2.56), à

$$
R=-\frac{12}{L^{2}}
$$

onde $L$ é o raio AdS, que está relacionado à constante cosmológica por $\Lambda_{4 D}=-3 / L^{2}$.

Para resolver (4.24), somente na brana, para o caso estacionário com simetria axial e constante cosmológica negativa, utilizamos um ansatz 4-dimensional escrito na forma KerrSchild-AdS, que pode ser encontrado em [8]:

$$
d s^{2}=d s_{A d S}^{2}+H\left(l_{\mu} d x^{\mu}\right)^{2}
$$

Neste caso, $d s_{A d S}^{2}$ é a métrica anti-de Sitter pura, $H$, novamente, é uma função de $r$ e $\theta$, e $l_{\mu}$ representa um vetor de norma nula. Nas coordenadas $(t, r, \theta, \phi),(4.25)$ apresenta-se como:

$$
d s_{A d S}^{2}=-\frac{\left(1+\frac{r^{2}}{L^{2}}\right) \Delta_{\theta}}{1-\frac{a^{2}}{L^{2}}} d t^{2}+\frac{\Sigma}{\left(1+\frac{r^{2}}{L^{2}}\right)\left(r^{2}+a^{2}\right)} d r^{2}+\frac{\Sigma}{\Delta_{\theta}} d \theta^{2}+\frac{\left(r^{2}+a^{2}\right) \operatorname{sen}^{2} \theta}{1-\frac{a^{2}}{L^{2}}} d \phi^{2}
$$

e

$$
H\left(l_{\mu} d x^{\mu}\right)^{2}=H\left(\frac{\Delta_{\theta}}{1-\frac{a^{2}}{L^{2}}} d t+\frac{\Sigma}{\left(1+\frac{r^{2}}{L^{2}}\right)\left(r^{2}+a^{2}\right)} d r-\frac{a \operatorname{sen}^{2} \theta}{1-\frac{a^{2}}{L^{2}}} d \phi\right)^{2}
$$

sendo

$$
\Delta_{\theta}=1-\frac{a^{2}}{L^{2}} \cos ^{2} \theta \quad \text { e } \quad \Sigma=r^{2}+a^{2} \cos ^{2} \theta .
$$

A constante $a$ foi interpretada como parâmetro de rotação - impondo-a nula, temos uma solução simetricamente esférica. Inserindo (4.26) e (4.27) na equação (4.24), obtivemos

$$
R=\frac{\partial^{2} H}{\partial r^{2}}+\frac{4 r}{\Sigma} \frac{\partial H}{\partial r}+\frac{2}{\Sigma} H-\frac{12}{L^{2}}
$$

ou seja,

$$
\frac{\partial^{2} H}{\partial r^{2}}+\frac{4 r}{\Sigma} \frac{\partial H}{\partial r}+\frac{2}{\Sigma} H=0 .
$$

Assim como [33], a equação (4.30) conduziu-nos a uma função $H(r, \theta)$ que não depende da 
constante cosmológica. Diferentemente de [8], $H(r, \theta)$ depende de uma constante de integração $M$ mais um termo novo, $q$, chamado de "carga de maré", tal como no caso assintoticamente plano. Isto é,

$$
H=\frac{2 M r}{\Sigma}-\frac{q}{\Sigma}
$$

Para obter algumas grandezas físicas relevantes, como o horizonte de eventos, passamos a métrica na forma Kerr-Schild-AdS, com o uso de (4.26) e (4.27), para as coordenadas de Boyer-Lindquist utilizando as transformações

$$
d t=d \tau+\frac{\Sigma H}{\left(1+\frac{r^{2}}{L^{2}}\right) \Delta_{r}} d r
$$

e

$$
d \phi=d \varphi+\frac{a}{L^{2}} d \tau+\frac{a \Sigma H}{\left(r^{2}+a^{2}\right) \Delta_{r}} d r
$$

onde

$$
\Delta_{r}=\left(r^{2}+a^{2}\right)\left(1+\frac{r^{2}}{L^{2}}\right)-2 M r+q .
$$

Nessas coordenadas, $q$ faz o papel de $Q^{2}$ da solução Kerr-Newman-AdS. Nesta última, $\Delta_{r}=\left(r^{2}+a^{2}\right)\left(1+r^{2} / L^{2}\right)-2 M r+Q^{2}$. Portanto, a solução obtida acima é uma solução estacionária na brana com simetria axial, constante cosmológica negativa e uma "carga" induzida, que chamamos de "carga de maré", assim como Aliev e Gümrükçüoglu na solução exposta na seção anterior. Assim fica claro, segundo (4.34), o porquê atribuirmos a palavra carga à constante $q$, mesmo assumindo um tensor eletromagnético nulo na brana $\left(F_{\mu \nu}=0\right)$. Esta constante, $q$, mede a influência do bulk na brana (veremos que a sua aparição nas expressões para os horizontes também nesse caso modifica o tamanho destes). Por fim, temos, a partir das transformações de coordenadas realizadas, a métrica na forma Boyer-Lindquist

$$
\begin{aligned}
d s^{2} & =-\frac{1}{\Sigma}\left(\Delta_{r}-\Delta_{\theta} a^{2} \operatorname{sen}^{2} \theta\right) d \tau^{2}-\frac{2 a}{\left(1-\frac{a^{2}}{L^{2}}\right) \Sigma}\left[\left(r^{2}+a^{2}\right) \Delta_{\theta}-\Delta_{r}\right] \operatorname{sen}^{2} \theta d \tau d \varphi+ \\
& +\frac{\Sigma}{\Delta_{r}} d r^{2}+\frac{\Sigma}{\Delta_{\theta}} d \theta^{2}+\frac{1}{\left(1-\frac{a^{2}}{L^{2}}\right)^{2} \Sigma}\left[\left(r^{2}+a^{2}\right)^{2} \Delta_{\theta}-\Delta_{r} a^{2} \operatorname{sen}^{2} \theta\right] \operatorname{sen}^{2} \theta d \varphi^{2}
\end{aligned}
$$

A métrica acima, nas coordenadas $(\tau, r, \theta, \varphi)$, possui problemas: diverge quando $\Sigma=$ 0 , ou $\Delta_{r}=0$, ou $\Delta_{\theta}=0$, ou $a^{2}=L^{2}$. Quando calculamos o escalar de Kretschmann, $R_{\alpha \beta \gamma \delta} R^{\alpha \beta \gamma \delta}$, demo-nos conta de que somente quando $\Sigma=0$ ou $\Delta_{\theta}=0$ temos um problema que não se deve ao sistema de coordenadas escolhido. Ou seja,

$$
R_{\alpha \beta \gamma \delta} R^{\alpha \beta \gamma \delta} \propto \frac{1}{\Sigma^{8}}+\frac{1}{\Delta_{\theta}} .
$$


Por (4.28), o escalar acima diverge, como na métrica de Kerr, quando $r=\cos \theta=0$. Assim, por ter a mesma simetria, interpretamos também aqui a singularidade física como um anel, uma estrutura como aquela existente em Kerr, comentada na seção 4.1. A divergência ocorre também quando $\Delta_{\theta}=0$, ou seja, $L^{2}=a^{2} \cos ^{2} \theta$. Este último resultado nos indicou que o raio $\mathrm{AdS}$ tem um tamanho mínimo - o que equivale à $\Lambda_{4 D}$ ter igualmente um valor mínimo $\left(\Lambda_{4 D}=-3 / L^{2}\right)$. Quando $\Delta_{\theta}=0$,

$$
\Lambda_{4 D}=-\frac{3}{a^{2} \cos ^{2} \theta} .
$$

Como $0 \leq \theta \leq \pi / 2$

$$
-\infty \leq \Lambda_{4 D} \leq-\frac{3}{a^{2}}
$$

Trabalhamos, então, somente com $\Lambda_{4 D}>-3 / a^{2}$ ou $L^{2}>a^{2}$. No caso assintoticamente dS da próxima seção, como veremos, esse problema não existe porque se trata de uma solução com constante cosmológica positiva.

A métrica (4.35) tem a mesma simetria e, por isso, pode ser escrita da mesma forma que a métrica de Kerr ou de Kerr-Newman. Devido a essa semelhança, a extensão da métrica (4.35) realiza-se, como no caso Kerr exposto na seção 4.1, com as transformações (4.6) e (4.8), tornando-a analítica quando $\Delta_{r}=0$.

Impondo $g^{r r}=0$, ou seja, $\Delta_{r}=0$, temos os horizontes - superfícies cujos vetores tangentes têm norma nula - como raízes desta equação, de acordo com o apêndice C. No caso da métrica (4.35), a equação resolvida foi um polinômio de quarto grau. Não expusemos tais raízes obtidas devido ao grande tamanho que cada expressão possui. Mas para o caso onde $a<M$, o único caso que abordamos, temos quatro raízes, sendo duas reais e duas complexas conjugadas, desde que $q_{\min }<q<q_{\max }$ (os valores de $q_{\min }$ e $q_{\max }$ dependem da escolha dos parâmetros $a, M, L)$. As raízes reais correspondem a um horizonte interno, $r_{-}$, e um horizonte externo, $r_{+}$. Este último pode ser considerado o horizonte de eventos usual. A constante $q$, novamente, influencia estes resultados - determinando diretamente os raios dos horizontes, de acordo com a figura 4.1. Um detalhe importante a enfatizar é que $q_{\min }$ pode assumir até valores negativos. Este resultado é uma inovação em relação à relatividade geral, pois na métrica de Kerr-Newman o termo da carga é expresso ao quadrado; não importa, então, se $Q$ é positiva ou negativa; em ambos os casos, os raios dos horizontes têm os mesmos comprimentos. Entretanto, para valores negativos de $q$, como fica claro na figura 4.1, o horizonte de eventos torna-se ainda maior.

Outro aspecto importante de (4.35) é variação do raio do horizonte de eventos com o aumento ou diminuição do parâmetro de rotação $a$. O gráfico 4.2 mostra que o raio do horizonte de eventos diminui com o aumento do parâmetro de rotação. 


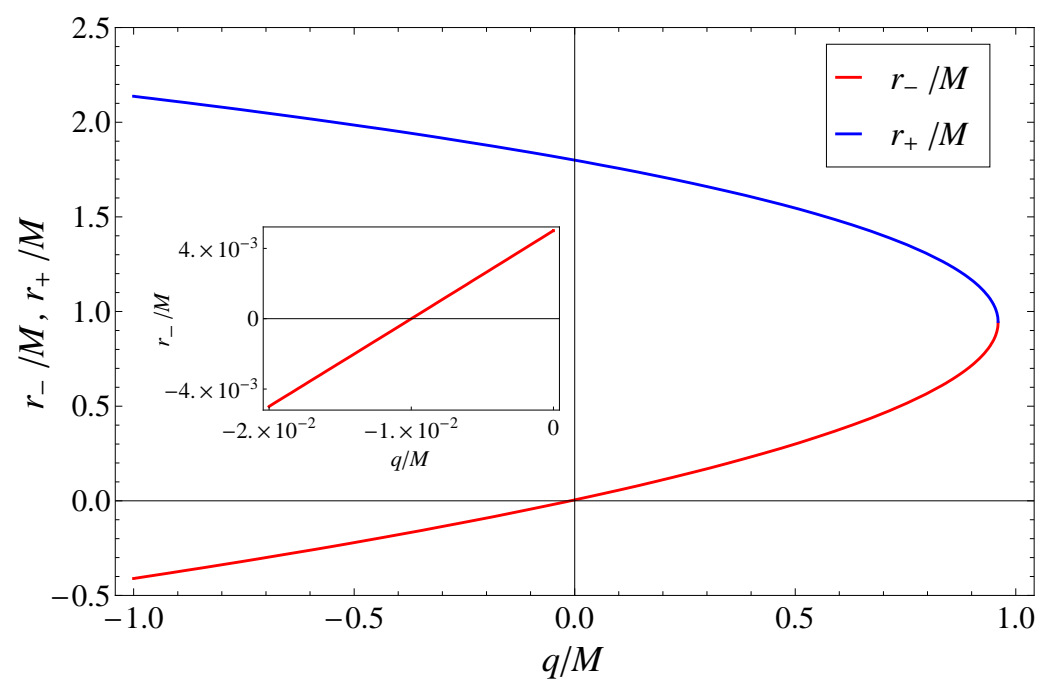

Figura 4.1: O horizonte interno, $r_{-}$, linha vermelha, e o horizonte de eventos, $r_{+}$, linha azul, são dependentes do parâmetro $q$, a "carga de maré". O gráfico interno representa a ampliação de um pequeno intervalo negativo para mostrar que para valores de $q<0$ temos $r_{-}$e $r_{+}$reais e positivos. Usamos neste gráfico $a=0.1 M, L=\sqrt{30} M$ e $M=1$.

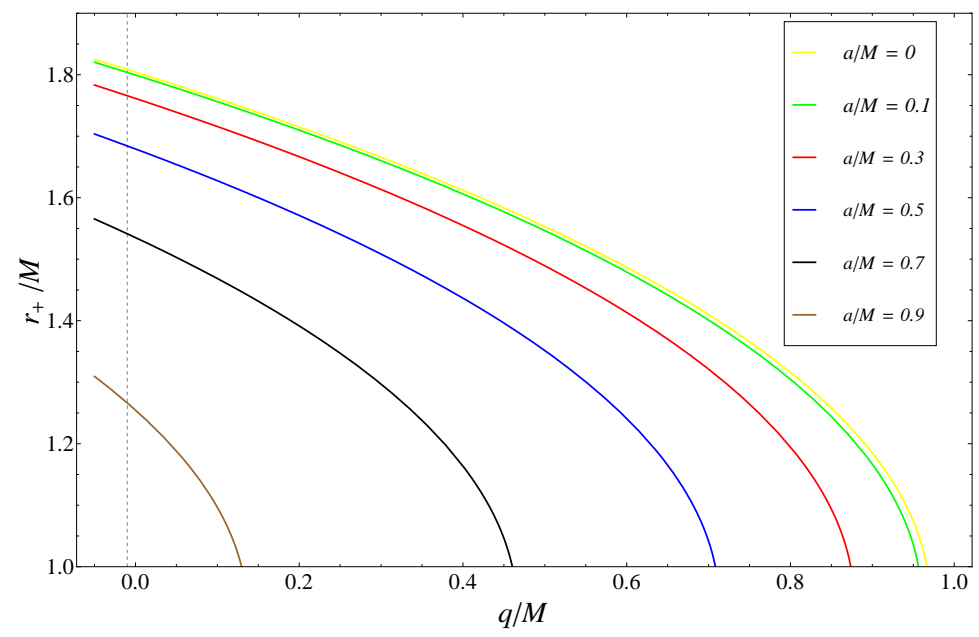

Figura 4.2: A dependência do tamanho ou raio do horizonte de eventos com o parâmetro de rotação, $a$, é ilustrada neste gráfico. Com o aumento desse parâmetro, o raio do horizonte diminui. Usamos neste gráfico $a=0.1 M, L=\sqrt{30} M$ e $M=1$. Neste gráfico, a linha vertical tracejada indica o valor mínimo de $q / M$ para que tenhamos dois horizontes.

A métrica (4.35) nas coordenadas $(\tau, r, \theta, \varphi)$ é independente de duas coordenadas: $\tau$ e $\varphi$; possui, consequentemente, 2 campos de Killing que podem ser imediatamente construídos na 
base das coordenadas ${ }^{14}$ :

$$
\xi_{\tau}=\frac{\partial}{\partial \tau} \quad \text { e } \quad \xi_{\varphi}=\frac{\partial}{\partial \varphi}
$$

Suas normas são

$$
\xi_{\tau}^{2}=g_{\tau \tau}=-\frac{1}{\Sigma}\left(\Delta_{r}-\Delta_{\theta} a^{2} \operatorname{sen}^{2} \theta\right)
$$

e

$$
\xi_{\varphi}^{2}=g_{\varphi \varphi}=\frac{1}{\left(1-\frac{a^{2}}{L^{2}}\right)^{2} \Sigma}\left[\left(r^{2}+a^{2}\right)^{2} \Delta_{\theta}-a^{2} \Delta_{r} \operatorname{sen}^{2} \theta\right] \operatorname{sen}^{2} \theta
$$

Os zeros da equação (4.40) fornecem as superfícies de Killing geradas por $\xi_{\tau}$, que nesse caso dependem de $M, a, \theta, L$ e $q$. Para valores de $q / M$ que nos permitem ter dois horizontes, podemos dividir o espaço-tempo em cinco regiões, de acordo com a figura 4.3:

$$
0<S_{-}<r_{-}<r_{+}<S_{+}<\infty
$$

Os dois zeros reais de $\xi_{\tau}^{2}, S_{-}$e $S_{+}$, são as duas superfícies onde o campo de Killing $\xi_{\tau}$ tem norma nula. A região entre $S_{-}$e $S_{+}$é especial: nela, a norma do campo de Killing $\xi_{\tau}$ é positiva, como mostra o gráfico 4.3. Isto é, na assinatura da métrica usada por nós, um vetor com norma positiva é do tipo espaço. Da mesma forma, na região entre $r_{+}$e $S_{+}$, $\xi_{\tau}$ é do tipo espaço; esta região tem um nome devido à sua importância - tal é conhecida como ergosfera, como já foi mencionada na seção 4.1. Outra característica desse tipo de geometrias assintoticamente AdS, como fica nítida no gráfico 4.3, é a norma do campo de Killing $\xi_{\tau}$ que diverge quando $r$ tende para o infinito.

Temos tratado a solução (4.35) como assintoticamente AdS. O nome desta seção torna isso ainda mais flagrante. Uma forma rápida de se convencer disso é olhar para a métrica na forma Kerr-Schild-AdS, especialmente no termo (4.27) que envolve $H(r, \theta)$. Quando $r$ tende ao infinito, esse termo tende a zero, devido à função $H(r, \theta)$ ser inversamente proporcional ao quadrado de $r$. Então,

$$
d s^{2} \rightarrow d s_{A d S}^{2} \text { quando } r \rightarrow \infty
$$

e a solução é assintoticamente anti-de Sitter.

\footnotetext{
${ }^{14}$ Sobre a relação entre campos de Killing e coordenadas, o apêndice B faz uma pequena exposição, relacionando tais campos com as simetrias esférica e axial.
} 

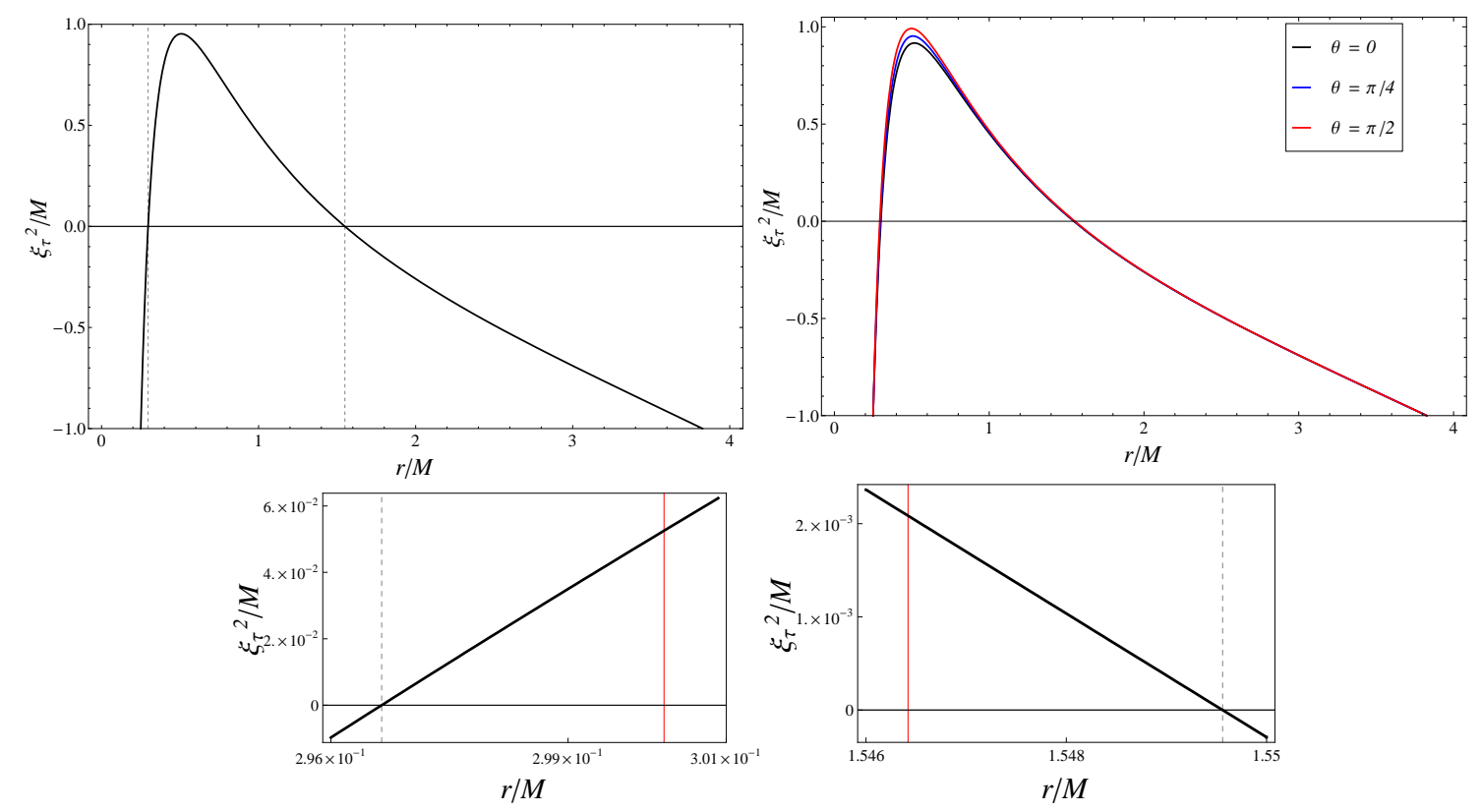

Figura 4.3: A norma do campo de Killing $\xi_{\tau}$ para o caso assintoticamente AdS é ilustrada nos gráficos superiores. As retas verticais tracejadas no gráfico superior esquerdo indicam as superfícies de Killing, $S_{-}$e $S_{+}$. No gráfico superior à direita, constatamos que para diferentes valores de $\theta$, o ângulo polar, a norma do campo tende, quando $r$ cresce, para o mesmo valor independentemente do valor do ângulo. Nos gráficos inferiores, destacamos regiões próximas às superfícies $S_{-}$e $S_{+}$(retas verticais tracejadas), respectivamente. As retas verticais em vermelho indicam o horizonte interno, na figura inferior à esquerda, e o horizonte de eventos, figura inferior à direita. Com isso, podemos dizer que o espaço-tempo é dividido em cinco regiões: $0<S_{-}<r_{-}<r_{+}<S_{+}<\infty$. Usamos nestes gráficos $a=0.1 M, L=\sqrt{30} M$ e $M=1$.

\subsection{Solução assintoticamente dS}

De forma similar ao caso da seção anterior, também obtivemos em [35] uma solução estacionária com simetria axial e constante cosmológica positiva num contexto de mundos brana. Para tal, resolvemos a equação (2.56). Entretanto, temos, agora, que impor a constante cosmológica positiva na brana. Ou seja,

$$
R=\frac{12}{L^{2}}
$$

onde $L$, neste caso, é o raio dS, que se relaciona com a constante cosmológica na brana por $\Lambda_{4 D}=3 / L^{2}$.

Seguindo os mesmos passos do caso AdS da seção anterior para resolver a equação dife- 
rencial (4.44), utilizamos o ansatz Kerr-Schild-dS, de acordo com [8]:

$$
d s^{2}=d s_{d S}^{2}+H\left(l_{\mu} d x^{\mu}\right)^{2},
$$

que nas coordenadas $t, r, \theta$ e $\phi$ tomou a forma

$$
d s_{d S}^{2}=-\frac{\left(1-\frac{r^{2}}{L^{2}}\right) \Delta_{\theta}}{1+\frac{a^{2}}{L^{2}}} d t^{2}+\frac{\Sigma}{\left(1-\frac{r^{2}}{L^{2}}\right)\left(r^{2}+a^{2}\right)} d r^{2}+\frac{\Sigma}{\Delta_{\theta}} d \theta^{2}+\frac{\left(r^{2}+a^{2}\right) \operatorname{sen}^{2} \theta}{1+\frac{a^{2}}{L^{2}}} d \phi^{2}
$$

$\mathrm{e}$

$$
H\left(l_{\mu} d x^{\mu}\right)^{2}=H\left(\frac{\Delta_{\theta}}{1+\frac{a^{2}}{L^{2}}} d t+\frac{\Sigma}{\left(1-\frac{r^{2}}{L^{2}}\right)\left(r^{2}+a^{2}\right)} d r-\frac{a^{2} e^{2} \theta}{1+\frac{a^{2}}{L^{2}}} d \phi\right)^{2}
$$

onde

$$
\Delta_{\theta}=1+\frac{a^{2}}{L^{2}} \cos ^{2} \theta \quad \text { e } \quad \Sigma=r^{2}+a^{2} \cos ^{2} \theta .
$$

Novamente, a constante $a$ será interpretada como parâmetro de rotação. Inserindo (4.46) e (4.47) na equação (4.44), tivemos

$$
R=\frac{\partial^{2} H}{\partial r^{2}}+\frac{4 r}{\Sigma} \frac{\partial H}{\partial r}+\frac{2}{\Sigma} H+\frac{12}{L^{2}}
$$

ou seja,

$$
\frac{\partial^{2} H}{\partial r^{2}}+\frac{4 r}{\Sigma} \frac{\partial H}{\partial r}+\frac{2}{\Sigma} H=0,
$$

que é a mesma equação obtida no caso AdS, cuja solução é dada por (4.31).

Passamos, igualmente para este caso, a métrica da forma Kerr-Schild-dS para as coordenadas de Boyer-Lindquist, de acordo com as transformações

$$
d t=d \tau+\frac{\Sigma H}{\left(1-\frac{r^{2}}{L^{2}}\right) \Delta_{r}} d r
$$

$\mathrm{e}$

$$
d \phi=d \varphi-\frac{a}{L^{2}} d \tau+\frac{a \Sigma H}{\left(r^{2}+a^{2}\right) \Delta_{r}} d r
$$

onde

$$
\Delta_{r}=\left(r^{2}+a^{2}\right)\left(1-\frac{r^{2}}{L^{2}}\right)-2 M r+q .
$$

Tal como o caso AdS, nessas coordenadas, $q$ faz o papel de uma "carga induzida", uma "carga de maré", e tem alguma semelhança com $Q^{2}$ da solução Kerr-Newman-dS, onde $\Delta_{r}=\left(r^{2}+a^{2}\right)\left(1-r^{2} / L^{2}\right)-2 M r+Q^{2}$. Então, temos uma solução com simetria axial, constante cosmológica positiva e uma "carga" induzida na brana. Por fim, a métrica, depois das 
transformações de coordenadas, apresentou-se como

$$
\begin{aligned}
d s^{2} & =-\frac{1}{\Sigma}\left(\Delta_{r}-\Delta_{\theta} a^{2} \operatorname{sen}^{2} \theta\right) d \tau^{2}-\frac{2 a}{\left(1+\frac{a^{2}}{L^{2}}\right) \Sigma}\left[\left(r^{2}+a^{2}\right) \Delta_{\theta}-\Delta_{r}\right] \operatorname{sen}^{2} \theta d \tau d \varphi+ \\
& +\frac{\Sigma}{\Delta_{r}} d r^{2}+\frac{\Sigma}{\Delta_{\theta}} d \theta^{2}+\frac{1}{\left(1+\frac{a^{2}}{L^{2}}\right)^{2} \Sigma}\left[\left(r^{2}+a^{2}\right)^{2} \Delta_{\theta}-\Delta_{r} a^{2} \operatorname{sen}^{2} \theta\right] \operatorname{sen}^{2} \theta d \varphi^{2} .
\end{aligned}
$$

A métrica acima, tal como a métrica assintoticamente $\mathrm{AdS}$ da seção anterior, diverge quando $\Delta_{r}=0$ ou $\Sigma=0$. Neste caso não existem valores de $L$ que a tornem divergente. Diferente daquela, esta métrica tem uma singularidade física somente quando $\Sigma=0$, como pode ser visto no escalar de Kretschmann:

$$
R_{\alpha \beta \gamma \delta} R^{\alpha \beta \gamma \delta} \propto \frac{1}{\Sigma^{8}}
$$

A divergência em $\Delta_{r}$ mostra-se apenas como uma insuficiência das coordenadas de BoyerLindquist na região da variedade onde isso ocorre. Da mesma maneira que a métrica da seção anterior, (4.54) também pôde ser escrita na forma em que as métricas de Kerr ou de Kerr-Newman são escritas, nas coordenadas de Boyer-Lindquist. Devido a essa semelhança, a extensão da métrica (4.54) realiza-se, como no caso Kerr exposto na seção 4.1, com as transformações (4.6) e (4.8), tornando-a analítica quando $\Delta_{r}=0$.

Vamos discutir os horizontes dessa métrica, que são um pouco diferentes do caso estudado na seção anterior. Impondo, novamente, $g^{r r}=0$, ou seja, $\Delta_{r}=0$, temos até quatro raízes diferentes para esse polinômio. Limitando-nos ao caso $a<M$, se $q_{\text {min }}<q<q_{\text {max }}$ - onde os valores de $q_{\min }$ e $q_{\max }$ dependem da escolha dos parâmetros $a, M, L-$, temos até três raízes reais, sendo a menor delas o horizonte interno $\left(r_{-}\right)$, a intermediária, o horizonte de eventos $\left(r_{+}\right)$, e a maior, o horizonte cosmológico $\left(r_{c}\right)$. Vemos que, neste caso, um horizonte a mais apresenta-se. Como no caso anterior, as expressões para esses horizontes são muito grandes para serem expostas aqui. Ilustramos seus comportamentos, suas dependências com o parâmetro $q$, a "carga de maré", com o auxílio da figura 4.4. Novamente, fica claro que $q$ afeta o tamanho dos horizontes de uma forma diferente da carga $Q$ na métrica Kerr-Newman — que se apresenta ao quadrado —, mostrando-nos um resultado qualitativamente distinto da relatividade geral. Quando a "carga de maré" assume valores negativos, o raio do horizonte de eventos adquire tamanho ainda maior - como pode ser notado no gráfico 4.4. Nos gráficos da figura 4.5, vemos que com o aumento do parâmetro de rotação $a$, para um mesmo valor de $q / M$, o raio do horizonte de eventos diminui, e o raio do horizonte cosmológico aumenta. Isso é uma propriedade já conhecida de raízes de polinômios ${ }^{15}$.

\footnotetext{
${ }^{15}$ A propriedade mencionada é uma das fórmulas de Girard-Newton-Viéte. Para um polinômio de grau $n$,
} 


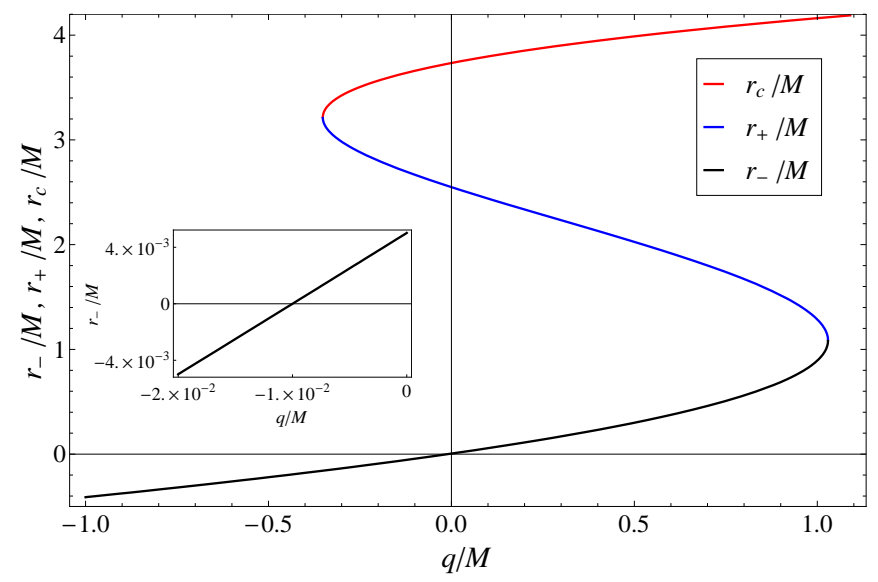

Figura 4.4: O horizonte interno, $r_{-}$, linha preta, o horizonte de eventos, $r_{+}$, linha azul, e o horizonte cosmológico, $r_{c}$, linha vermelha, são dependentes do parâmetro $q$, a "carga de maré". O gráfico menor, interno, destaca um pequeno intervalo negativo para mostrar que existe, mesmo para $q<0$, um valor real e positivo para $r_{-}, r_{+}$e $r_{c}$. Usamos neste gráfico $a=0.1 M, L=\sqrt{30} M$ e $M=1$.
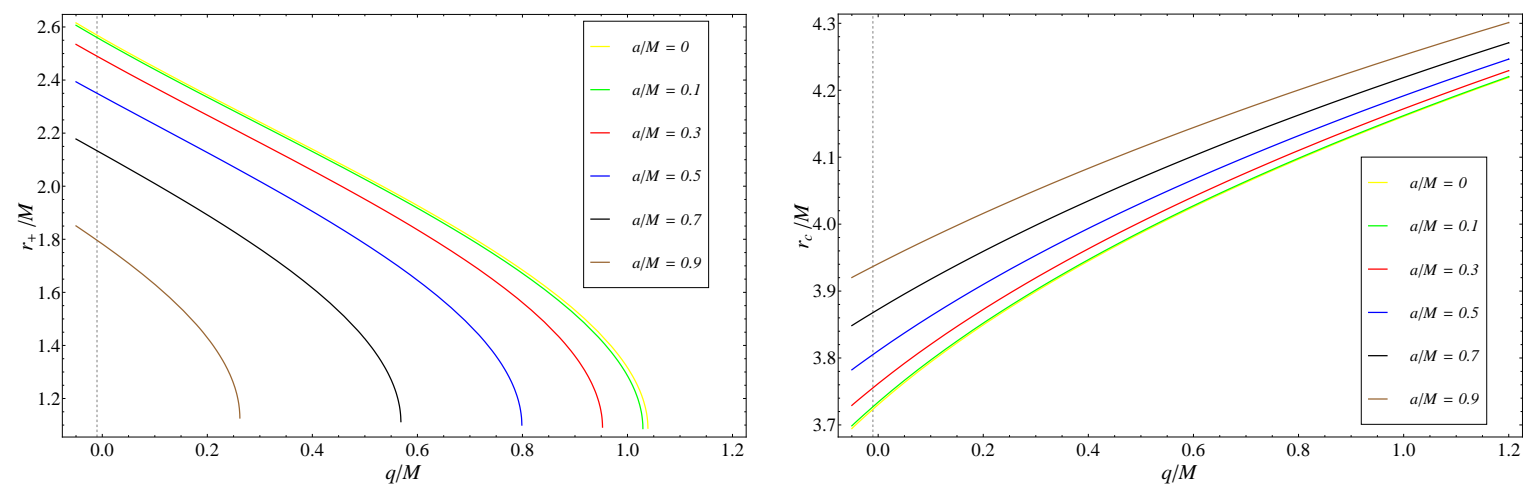

Figura 4.5: A variação dos horizontes de evento, $r_{+}$, e cosmológico, $r_{c}$, com o aumento da "carga de maré" para alguns valores do parâmetro de rotação é exibida nas figuras da esquerda e da direita, respectivamente. Com o aumento de $a$, para um mesmo valor de $q$, o horizonte de eventos diminui e o cosmológico aumenta. Usamos nestes gráficos $a=0.1 M, L=\sqrt{30} M$ e $M=1$.

Tal como a métrica assintoticamente AdS, a métrica desta seção nas coordenadas $(\tau, r, \theta, \varphi)$ é independente de duas coordenadas: $\tau$ e $\varphi$; possui, da mesma forma, 2 campos de Killing que podem ser imediatamente construídos na base das coordenadas como (4.39), cujas normas são

$$
\xi_{\tau}^{2}=g_{\tau \tau}=-\frac{1}{\Sigma}\left(\Delta_{r}-\Delta_{\theta} a^{2} \operatorname{sen}^{2} \theta\right)
$$

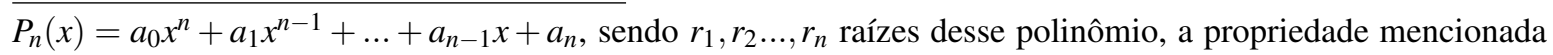
diz que: $r_{1}+r_{2}+\ldots+r_{n}=-a_{1} / a_{0}$. 
$\mathrm{e}$

$$
\xi_{\varphi}^{2}=g_{\varphi \varphi}=\frac{1}{\left(1+\frac{a^{2}}{L^{2}}\right)^{2} \Sigma}\left[\left(r^{2}+a^{2}\right)^{2} \Delta_{\theta}-a^{2} \Delta_{r} \operatorname{sen}^{2} \theta\right] \operatorname{sen}^{2} \theta
$$

Os zeros da equação (4.56) fornecem as superfícies de Killing, que nesse caso também dependem de $M, a, \theta, L$ e $q$. Para valores de $q / M$ que nos permitem ter três horizontes $\left(r_{-}, r_{+} \mathrm{e}\right.$ $r_{c}$ ), podemos dividir o espaço-tempo em seis regiões, de acordo com a figura 4.6:

$$
0<S_{-}<r_{-}<r_{+}<S_{i}<S_{+}<r_{c}
$$

Aqui, $\xi_{\tau}^{2}$ pode ter até três raízes: $S_{-}, S_{i}$ (uma superfície interna) e $S_{+}$, isto é, este campo assume norma nula em três superfícies. A região entre $S_{i}$ e $S_{+}$é especial. Nela a norma do campo de Killing $\xi_{\tau}$ é negativa, como mostra o gráfico 4.6. Isto é, na assinatura da métrica usada por nós, para constante cosmológica positiva, um vetor com norma negativa é do tipo espaço. De forma diferente do caso assintoticamente AdS, toda a região entre $r_{+}$e $S_{+}$não torna o campo $\xi_{\tau}$ tipo espaço. Isto só ocorre entre $S_{i}$ e $S_{+}$. Entre $r_{+}$e $S_{i}$, a norma do campo em questão é tipo tempo. Então, nesse caso, a ergosfera situa-se entre $S_{i}$ e $S_{+}$e não alcança o horizonte de eventos. Outra característica compartilhada com o caso AdS é a divergência da norma do campo $\xi_{\tau}$ quando $r$ cresce - nítida no gráfico 4.6.

Tal como a solução assintoticamente AdS da seção anterior, a métrica desta seção também tem um comportamento assintótico que não é de uma métrica plana. Por (4.45), tomando $r \rightarrow \infty$ (nas forma de Kerr-Schild-dS, a métrica pode ser estendida até o infinito), $H \rightarrow 0$ e nos conduz ao resultado

$$
d s^{2} \rightarrow d s_{d S}^{2}
$$

Portanto, podemos concluir que a métrica construída nesta seção é assintoticamente de Sitter.

As soluções (4.35) e (4.54) podem ser interpretadas de duas formas: no contexto da relatividade geral, temos soluções das equações de Einstein com um tensor energia-momento efetivo $T_{\mu \nu}^{e f}$ não nulo, pois podemos considerar que o tensor $E_{\mu \nu} / 8 \pi$ faz esse papel, na equação do campo gravitacional, em ambas soluções. Isto é,

$$
G_{\mu v}+\Lambda_{4 D} g_{\mu v}=-E_{\mu v}=8 \pi T_{\mu \nu}^{e f}
$$

Por outro lado, no contexto de mundos brana que temos tratado, consideramos vácuo na 3brana. Nesta interpretação, as equações do campo gravitacional são outras, são deduzidas a partir das equações de Gauss-Codazzi, como pode ser visto no apêndice A. Nesta outra formulação das equações do campo gravitacional, o tensor $E_{\mu \nu}$ é visto como a manifestação do bulk na brana, como um elemento que não tem correspondente na relatividade geral. De qualquer forma, em ambas interpretações e soluções (assintoticamente AdS e dS) as componentes 
$E_{\mu}^{\mu}$ são:

$$
\begin{aligned}
& E_{\tau}^{\tau}=-E_{\varphi}^{\varphi}=q\left(\frac{2\left(r^{2}+a^{2}\right)}{\Sigma^{3}}-\frac{1}{\Sigma^{2}}\right) \\
& E_{r}^{r}=-E_{\theta}^{\theta}=\frac{q}{\Sigma^{2}} \\
& E_{\varphi}^{\tau}=-\frac{\left(r^{2}+a^{2}\right) \operatorname{sen}^{2} \theta}{\Xi} E_{\tau}^{\varphi}=-\frac{2 q a}{\Xi \Sigma^{3}}\left(r^{2}+a^{2}\right) \operatorname{sen}^{2} \theta
\end{aligned}
$$

onde

$$
\Xi=1+\frac{\Lambda_{4 D}}{3} a^{2}
$$

Pode-se notar que o vínculo $E_{\mu}^{\mu}=0$ é satisfeito.
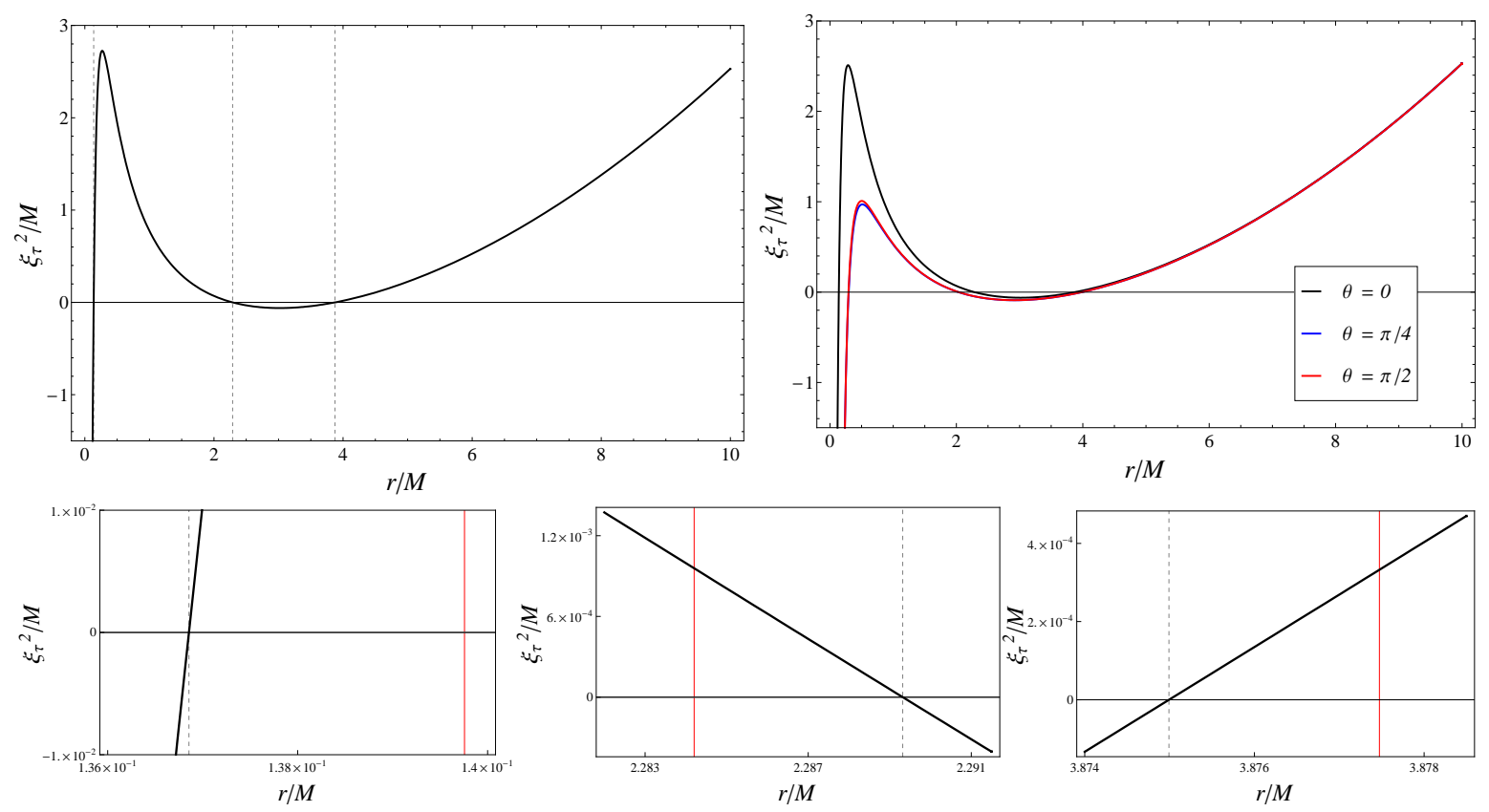

Figura 4.6: A norma do campo de Killing $\xi_{\tau}$ para o caso assintoticamente dS é ilustrada nos gráficos superiores. As retas verticais tracejadas no gráfico superior esquerdo indicam as superfícies de Killing, $S_{-}, S_{i}$ e $S_{+}$. No gráfico superior à direita, constatamos que para diferentes valores de $\theta$, o ângulo polar, a norma do campo tende, quando $r$ cresce, para o mesmo valor independentemente do valor do ângulo (uma grande diferença na curva é notada quando $\theta=0$, entre $S_{-}$e $S_{i}$ ). Nos gráficos inferiores, destacamos regiões próximas às superfícies $S_{-}, S_{i}$ e $S_{+}$(retas verticais tracejadas), respectivamente. As retas verticais em vermelho indicam o horizonte interno, na figura inferior à esquerda, o horizonte de eventos, figura inferior ao centro, e o horizonte cosmológico, figura inferior à direita. Com isso, podemos dizer que o espaço-tempo é dividido em seis regiões: $0<S_{-}<r_{-}<r_{+}<S_{i}<S_{+}<r_{c}$. Usamos nestes gráficos $a=0.1 M, L=\sqrt{30} M$ e $M=1$.

As componentes de $E_{\mu \nu}$ têm grande semelhança com as componentes do tensor energiamomento das soluções com carga elétrica - Kerr-Newman-(A)-dS e Kerr-Newman (quando 
$\Lambda_{4 D}=0$ ). Para estas métricas conhecidas, as componentes não nulas do tensor energiamomento são:

$$
\begin{aligned}
T_{\tau}^{\tau} & =-T_{\varphi}^{\varphi}=-\frac{Q^{2}}{8 \pi}\left(\frac{2\left(r^{2}+a^{2}\right)}{\Sigma^{3}}-\frac{1}{\Sigma^{2}}\right), \\
T_{r}^{r} & =-T_{\theta}^{\theta}=-\frac{Q^{2}}{8 \pi \Sigma^{2}}, \\
T_{\varphi}^{\tau} & =-\frac{\left(r^{2}+a^{2}\right) \operatorname{sen}^{2} \theta}{\Xi} T_{\tau}^{\varphi}=\frac{Q^{2} a}{4 \pi \Xi \Sigma^{3}}\left(r^{2}+a^{2}\right) \operatorname{sen}^{2} \theta .
\end{aligned}
$$

Sendo assim, a semelhança entre as componentes (4.61) e (4.63) é patente; basta trocar $q$ por $Q^{2}$ e multiplicar $E_{\mu v}$ por $-1 / 8 \pi$ para termos o tensor energia-momento do campo eletromagnético. Isto torna razoável interpretar $q$, nas soluções obtidas, como um tipo de carga coulombiana.

Quando interpretamos as soluções construídas nas duas seções anteriores como buracos negros assintoticamente AdS e dS (e temos razões para isso, pois tais apresentam todas as estruturas das soluções Kerr-Newman-(A)dS, como o horizonte de eventos, horizontes de Killing, ergosfera), podemos estimar a velocidade angular desses objetos como

$$
\Omega=-\frac{g_{\tau \varphi}}{g_{\varphi \varphi}}=-a \Xi\left(\frac{\Delta_{r}-\Delta_{\theta}\left(r^{2}+a^{2}\right)}{\Delta_{\theta}\left(r^{2}+a^{2}\right)^{2}-\Delta_{r} a^{2} \operatorname{sen}^{2} \theta}\right) .
$$

No "infinito", para o caso AdS, quando $r \rightarrow \infty$, um observador mede

$$
\Omega_{\infty}=\frac{\Lambda_{4 D}}{3} a,
$$

que, ao contrário da métrica de Kerr $\left(\Lambda_{4 D}=0\right)$, não é nula. Assim, um espaço-tempo assintoticamente AdS diferencia-se de um espaço-tempo assintoticamente plano também por essa característica. No horizonte, $\Delta_{r}=0$, a velocidade angular desses buracos negros tornou-se

$$
\Omega_{+}=\frac{a \Xi}{r_{+}^{2}+a^{2}} .
$$

No caso dS, existe também a velocidade angular do horizonte cosmológico, dada por

$$
\Omega_{c}=\frac{a \Xi}{r_{c}^{2}+a^{2}} .
$$

Os resultados, quando $\Lambda_{4 D}<0$, das velocidades angulares acima são iguais aos mostrados em $[77,58]$ para o caso Kerr-AdS ou Kerr-Newman-AdS. Também concordam quando $\Lambda_{4 D}>0$, 
de acordo com [59] e com a definição de velocidade angular do horizonte cosmológico, que se encontra em [51], onde expuseram tais grandezas para a métrica Kerr-Newman-dS.

A partir do que foi visto nas duas seções anteriores e nesta, não importa se existe na brana a constante cosmológica, como nas geometrias construídas por nós, ou não, como na solução proposta por Aliev e Gümrükçüoglu. O bulk, que é assintoticamente AdS, imprime suas pegadas na brana. Estas pegadas, como vimos, alteram a estrutura da geometria do espaço-tempo 4-dimensional.

\subsection{Cargas conservadas}

Como já dissemos, as soluções (4.35) e (4.54) podem ser interpretadas de duas formas. Na primeira - no contexto de mundos brana que abordamos — , a formulação de uma termodinâmica completa apresenta-se problemática, pois a solução total (bulk + brana) nos é desconhecida. Na segunda - interpretando as soluções obtidas como soluções no contexto da teoria de Einstein —, uma termodinâmica é direta. De acordo com (4.61) e (4.63), o tensor energia-momento efetivo das soluções por nós construídas é do tipo Kerr-Newman-(A)-dS. A diferença fundamental faz-se notar na presença do termo de carga, que não está elevado ao quadrado. Impondo nas métricas construídas valores negativos para essa constante, como já vimos, nas seções 4.3 e 4.4, efeitos gravitacionais ampliam-se (com $q<0$, temos horizontes de eventos e ergosferas maiores, por exemplo). Então, a possibilidade de utilizar valores negativos para $q$ pode apresentar uma termodinâmica diferente dos casos Kerr-Newman-dS e Kerr-Newman-AdS.

Mas para construir uma termodinâmica, cargas conservadas são imprescindíveis. Cargas conservadas estão entre as mais importantes quantidades que uma teoria pode prover. Nas seções anteriores, evitamos chamar o parâmetro $M$ nas métricas (4.35) e (4.54) de massa ou de uma quantidade conservada outra qualquer. Em geometrias assintoticamente de Sitter e anti-de Sitter — no contexto da relatividade geral — , a determinação de uma quantidade conservada, como a massa de um buraco negro, não é um consenso. Por outro lado, em geometrias assintoticamente planas, as chamadas integrais de Komar são um caminho para obter cargas conservadas, como a massa e o momento angular. Quando uma geometria possui um campo de Killing do tipo tempo $\left(\xi_{\tau}\right)$, a integral de Komar, algo similar à integral de Gauss no eletromagnetismo, fornece a massa/energia do sistema:

$$
E=\frac{1}{4 \pi} \int_{\partial \Sigma} d^{2} x \sqrt{-\gamma^{(2)}} \eta_{\mu} \sigma_{\nu} \nabla^{\mu} \xi_{\tau}^{v}
$$

onde $\partial \Sigma$, uma 2-esfera no infinito espacial, é a fronteira de uma hipersuperfície do tipo es- 
paço, e $\gamma^{(2)}$ é o determinante de sua métrica induzida; os vetores unitários $\eta_{\mu}$ e $\sigma_{\nu}$ são normais às hipersuperfícies $\Sigma$ e $\partial \Sigma$, respectivamente. No caso da métrica de Kerr, por exemplo, $E=M$. Para calcular o momento angular, $J$, a integral de Komar correspondente leva em consideração o campo de Killing do tipo espaço $\xi_{\varphi}$, que caracteriza a rotação. Ou seja,

$$
J=-\frac{1}{8 \pi} \int_{\partial \Sigma} d^{2} x \sqrt{-\gamma^{(2)}} \eta_{\mu} \sigma_{\nu} \nabla^{\mu} \xi_{\varphi}^{\nu}
$$

Em Kerr, $J=a M$, onde $a$ é o parâmetro de rotação.

Em outro caminho, na formulação hamiltoniana da relatividade geral, o formalismo ADM (Arnowitt, Deser e Misner) fornece uma maneira para se calcular quantidades conservadas. Em uma geometria assintoticamente plana, cargas conservadas são justamente aquelas fornecidas pelo formalismo ADM, correspondendo às $d(d+1) / 2$ cargas relacionadas aos geradores do grupo de Poincaré em $d$ dimensões. Partindo de uma maneira de escrever a métrica tal como em teoria de perturbação (na aproximação linear)

$$
g_{\mu v}=\eta_{\mu v}+h_{\mu v}
$$

onde aqui $h_{\mu \nu}$ é requerida ser pequena somente no infinito espacial, não necessariamente em todo espaço-tempo, a massa/energia no formalismo ADM é dada por uma integral sobre uma 2-esfera no infinito espacial

$$
E_{A D M}=\frac{1}{16 \pi} \int_{\partial \Sigma} d^{2} x \sqrt{-\gamma^{(2)}} \sigma^{i}\left(\partial_{j} h_{i}^{j}-\partial_{i} h_{j}^{j}\right) .
$$

Os índices $i$ e $j$ referem-se a quantidades na 2-esfera. A integral de Komar (4.68) e a expressão para a massa ADM (4.71) concordam entre si quando $h_{\mu v}$ é independente do tempo no infinito.

Mas em geometrias assintoticamente de Sitter ou anti-de Sitter, (4.68) ou (4.69) e (4.71) divergem. No caso das integrais de Komar, podemos interpretar essa divergência pensando que as integrais acima são definidas em todo espaço. Então, em geometrias com $\Lambda<0$ ou $\Lambda>0$, a quantidade de energia levada em conta na integração - uma integral onde o infinito é um dos seus limites - é infinita porque a constante cosmológica pode ser encarada como uma componente da energia do espaço-tempo, permeando-o em sua totalidade. Para superar essa dificuldade, foram desenvolvidas as técnicas conforme e dos contratermos ${ }^{16}$. A primeira, devido a Penrose, utiliza-se de condições de contorno que lidam com o comportamento assintótico da geometria para remover as divergências; em geometrias assintoticamente AdS

\footnotetext{
${ }^{16}$ Uma tentativa de utilizar as integrais de Komar, em espaços-tempo assintoticamente AdS, evitando as divergências, foi feita, por exemplo, em [52].
} 
foi usada por Ashtekar e Magnon [53], para o caso quadrimensional, e por Ashtekar e Das [54], para dimensões maiores do que quatro. A segunda técnica - desenvolvida para geometrias assintoticamente anti-de Sitter [55] e para geometrias assintoticamente dS [56] surgiu de forma independente graças à correspondência AdS/CFT: nesse caso, contratermos são adicionados à ação gravitacional, a fim de torná-la finita, para promover a remoção das divergências em cálculo de quantidades conservadas. Entretanto, outro caminho, anterior à técnica dos contratermos, foi percorrido por Brown e York [57]. Os resultados obtidos por Brown e York são em relação a um espaço-tempo referência, considerado como "estado fundamental" à classe de geometrias em questão. Isto é, realiza-se, nesse caso, uma subtração entre a ação gravitacional e a ação do espaço-tempo que serve como referência - o "estado fundamental" —, que é um funcional da métrica induzida na fronteira do espaço tempo em questão, seja assintoticamente AdS ou dS. A escolha desse espaço-tempo referencial é colocada como um dos problemas da técnica de Brown e York. Mas a técnica dos contratermos é uma extensão da técnica dos dois últimos autores citados, eliminando a necessidade de imergir a geometria estudada num espaço-tempo que serve como referência.

Nesta tese, vamos seguir a técnica de adicionar contratermos à ação gravitacional. Em Themodynamics of Kerr-Newman-AdS black holes and conformal field theories [58], Caldarelli, Cognola e Klemm estudaram a termodinâmica da geometria de Kerr-Newman-AdS, obtendo a temperatura Hawking, uma expressão para a massa/energia (a fórmula de Smarr) e a entropia, com o auxílio de um contratermo à ação gravitacional. Já em The thermodynamic of a Kerr-Newman-de Sitter black hole [59], Dehghani e KhajehAzad usaram o mesmo procedimento, agora para a geometria com constante cosmológica positiva, para estudar o sistema que é composto por dois horizontes: o horizonte de eventos e o cosmológico ${ }^{17}$. Em ambos trabalhos, ação total é dada por

$$
S=S_{G}+S_{c t},
$$

onde $S_{G}$ é a ação gravitacional de Einstein-Hilbert com o termo de Hawking-Gibbons [61]. O segundo termo do lado direito de (4.72) é um contratermo adicionado à ação. Em nosso caso, a ação gravitacional é

$$
S_{G}=-\frac{1}{16 \pi} \int_{\mathscr{M}} d^{4} x \sqrt{-g}\left(R-2 \Lambda_{4 D}\right)-\frac{1}{16 \pi} \int_{\mathscr{M}} d^{4} x \mathscr{L}_{\text {campo }}+\frac{1}{8 \pi} \int_{\partial \mathscr{M}} d^{3} x \sqrt{-\gamma} K,
$$

onde $\mathscr{M}$ é o espaço-tempo, $g$ é o determinante de $g_{\mu \nu}, \mathscr{L}_{\text {campo }}$ é a lagrangiana do campo

\footnotetext{
${ }^{17}$ Termodinâmica em geometrias assintoticamente dS que contém um buraco negro apresentam um interessante problema sobre a estabilidade do sistema horizonte de eventos-horizonte cosmológico. Em [60] existe uma discussão sobre tal problema.
} 
tipo Maxwell, expresso pelo tensor energia-momento efetivo (4.61); $\partial \mathscr{M}$ é uma possível fronteira tridimensional de $\mathscr{M}$, com métrica induzida $\gamma_{i j}$ e curvatura extrínseca $K_{i j}$, sendo $K$ o traço desta última. A componente de ação que traz o(s) contratermo(s) - de acordo com Caldarelli et al. (no caso assintoticamente AdS) e Dehghani et al. (no caso assintoticamente dS) - escreve-se como:

$$
S_{c t}=\frac{1}{8 \pi} \int_{\partial \mathscr{M}} d^{3} x \sqrt{-\gamma}\left(\frac{2}{L} \pm \frac{L}{2} \mathscr{R}\right)
$$

Na ação acima, o sinal positivo em \pm indica o caso AdS, o negativo, $\mathrm{dS}^{18}$, e o escalar $\mathscr{R}$ é a curvatura intrínseca da fronteira, isto é, o escalar de Ricci associado à métrica induzida na fronteira.

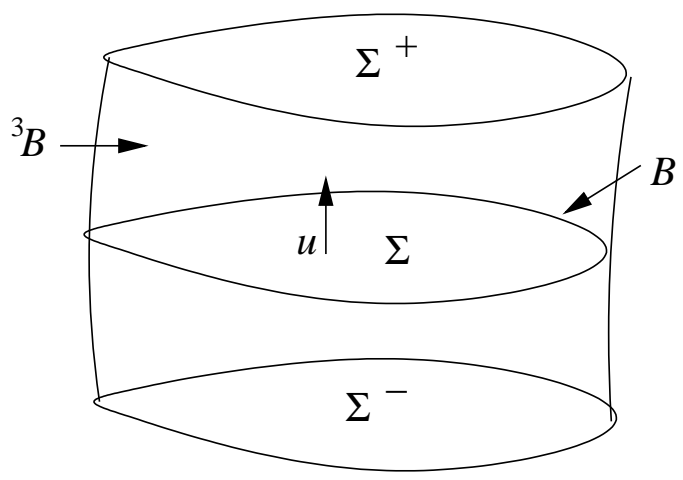

Figura 4.7: O espaço-tempo $\mathscr{M}$, no formalismo de Brown e York, tem fronteira dada por $\partial \mathscr{M}$. Esta última é definida pelas hipersuperfícies $\Sigma^{-}$, no passado, e $\Sigma^{+}$, no futuro, mais a hipersuperfície tipo tempo ${ }^{3} B$. Para uma hipersuperfície qualquer $\Sigma$, com $u$ sendo normal e unitário, sua fronteira é $B$.

Com a ação definida em (4.72), usa-se o caminho percorrido por Brown e York para construir quantidades conservadas ${ }^{19}$, definindo o tensor energia-momento na fronteira. $\mathrm{Ou}$ seja,

$$
\tau^{i j}=\frac{2}{\sqrt{-\gamma}} \frac{\delta S}{\delta \gamma_{i j}}
$$

determina quantidades conservadas em todo espaço-tempo $\mathscr{M}$. Extremizando $S, \tau^{i j}$ assume a forma

$$
\tau^{i j}=-\frac{1}{8 \pi}\left[\left(K^{i j}-K \gamma^{i j}\right) \pm \frac{2}{L} \gamma^{i j}-L\left(\mathscr{R}^{i j}-\frac{1}{2} \gamma^{i j} \mathscr{R}\right)\right]
$$

Mas antes, para obter as quantidades conservadas, escreve-se a métrica $\gamma_{i j}$ para tempos iguais

\footnotetext{
${ }^{18}$ Em Cadarelli et al.[58], existem mais termos em $S_{c t}$, mas segundo Dehghani et al. [59], tais termos a mais não contribuem devido aos seus decaimentos, quando $r$ cresce, serem mais rápidos.

${ }^{19}$ Vale a pena enfatizar que em [57], Brown e York não utilizam contratermos em sua ação total.
} 
no formalismo ADM. Isto é

$$
\gamma_{i j} d x^{i} d x^{j}=N^{2} d \rho^{2}+\sigma_{a b}\left(d \phi^{a}+V^{a} d \rho\right)\left(d \phi^{b}+V^{b} d \rho\right)
$$

com os índices $a$ e $b$ referindo-se a uma hipersuperfície de dimensão dois (indicada por $B$ na figura 4.7), cuja métrica induzida é expressa por $\sigma_{a b}$; as coordenadas $\phi^{a}$ são variáveis angulares que parametrizam curvas no entorno de uma origem. De acordo com o formalismo ADM, $N$ é a função lapso, e $V^{a}$ é o vetor de deslocamento. Dessa forma, para um campo de Killing $\xi$ associado a uma isometria na fronteira $\partial \mathscr{M}$, sua quantidade conservada correspondente é

$$
Q_{\xi}=\int_{B} d^{2} x \sqrt{\sigma} \tau^{i j} u_{i} \xi_{j}
$$

onde $u_{i}$ é um vetor normal unitário a uma superfície definida por $\rho$ fixo (indicada por $\Sigma$ na figura 4.7).

A determinação da quantidade conservada $M$, massa, ou momento angular, $J$, depende do uso em (4.78) do campo de Killing $\xi_{\tau}$ ou $\xi_{\varphi}$, respectivamente. Para Caldarelli et al. que usaram a métrica Kerr-Newman-AdS - , o resultado para a massa e o momento angular foram

$$
M_{h}=\frac{M}{\Xi^{2}} \quad \text { e } \quad J_{h}=\frac{M a}{\Xi^{2}} .
$$

Já para Dehghani et al. — que usaram a métrica Kerr-Newman-dS —, a massa e o momento angular foram calculados em relação ao horizonte cosmológico. Ou seja,

$$
M_{c}=-\frac{M}{\Xi^{2}} \quad \text { e } \quad J_{c}=-\frac{M a}{\Xi^{2}} .
$$

Por outro lado, em [62], seguindo o caminho de Gomberoff e Teitelboim [63], essas duas grandezas na geometria Kerr-Newman-dS foram também construídas para o horizonte de eventos. Nesse caso, os resultados são os mesmos da geometria assintoticamente AdS, dados por (4.79).

Como as métricas (4.35) e (4.54) são do tipo Kerr-Newman-dS e Kerr-Newman-AdS, podemos, no contexto da relatividade geral, interpretar a constante de integração $M$ como a massa do buraco negro multiplicada por um fator que depende somente da rotação e da constante cosmológica. Além disso, o momento angular também pode ser expresso por (4.79) ou (4.80), onde $a$ é interpretado como o parâmetro de rotação. 


\subsection{Geodésicas}

Segundo Chandrasekhar, no seu The mathematical theory of black holes, “(...) a separabilidade da equação de Hamilton-Jacobi (descoberta por Carter) foi a primeira de muitas propriedades que dotou a métrica de Kerr de uma aura miraculosa." ${ }^{20}$ Sem entrar na discussão sobre essa aura miraculosa da métrica de Kerr, construímos as equações das geodésicas — das métricas obtidas nas seções 4.3 e 4.4 - seguindo o mesmo caminho do seminal trabalho de Carter, Global structure of the Kerr family gravitational fields, publicado em 1968 [64]. As soluções construídas nas seções 4.3 e 4.4 têm duas constantes de movimento relacionadas aos campos de Killing $\xi_{\tau}$ e $\xi_{\varphi}$. A primeira representa a energia por unidade de massa, $E$, e a segunda representa o momento angular por unidade de massa, $\Phi$, de uma partícula em movimento em qualquer uma das duas geometrias com simetria axial expostas nas seções citadas. Duas quantidades conservadas a mais surgiram, sendo a última a partir da resolução da equação de Hamilton-Jacobi.

Num espaço-tempo, uma partícula somente sujeita à interação gravitacional percorre geodésicas, que na base das coordenadas são curvas que obedecem a equação (3.68). Geodésicas extremizam o comprimento de curvas que ligam dois pontos quaisquer. Sendo assim, pode-se obter (3.68) a partir da lagrangiana

$$
\mathscr{L}=\frac{1}{2} g_{\mu v} \dot{x}^{\mu} \dot{x}^{v}
$$

onde o ponto indica derivada ordinária em relação a um parâmetro, $\mu$, que se relaciona com o parâmetro afim $\lambda$, segundo Carter, por

$$
\lambda=\delta \mu
$$

Isto é equivalente a impor a seguinte condição de normalização

$$
g_{\mu v} \dot{x}^{\mu} \dot{x}^{v}= \pm \delta^{2}
$$

O sinal \pm na condição (4.83) refere-se ao caso de Sitter e anti-de Sitter, respectivamente. Como foi visto, no caso de Sitter, um vetor tipo tempo tem norma positiva, no outro, tem norma negativa. Escolhendo $\delta=0$ ou $\delta=1$, a lagrangiana (4.81) fornece geodésicas do tipo luz ou do tipo tempo, respectivamente. A equação (4.83) é conhecida como condição de normalização e fornece uma terceira constante de movimento, $\delta$, ligada à conservação da norma do vetor tangente paralelamente transportado ao longo da geodésica - a quadrivelocidade.

\footnotetext{
${ }^{20}$ Cf. [65], cap. 7, p. 319.
} 
A quarta constante de movimento para as geometrias expostas nas seções anteriores pôde ser obtida, como já dissemos, seguindo o caminho de Carter [64], num processo de separação de variáveis realizado para a resolução da equação de Hamilton-Jacobi.

Antes, construímos a hamiltoniana usando a definição dos momentos generalizados para a lagrangiana (4.81). Ou seja,

$$
p_{\mu} \equiv \frac{\partial \mathscr{L}}{\partial \dot{x}^{\mu}}=g_{\mu v} \dot{x}^{v}
$$

conduziu-nos à

$$
H \equiv p_{\mu} \dot{x}^{\mu}-\mathscr{L}=\frac{1}{2} g^{\mu v} p_{\mu} p_{v}
$$

para a nossa lagrangiana (com $\left.p^{\mu}=\dot{x}^{\mu}\right)$. Os momentos conjugados, usando as métricas ${ }^{21}$ assintoticamente de Sitter e anti-de Sitter construídas nas seções 4.3 e 4.4, são:

$$
\begin{aligned}
p_{t} & =g_{t t} \dot{t}+g_{t \varphi} \dot{\varphi} \\
& =-\frac{1}{\Sigma}\left(\Delta_{r}-\Delta_{\theta} a^{2} \operatorname{sen}^{2} \theta\right) \dot{t}-\frac{a}{\left(1+\frac{\Lambda_{4 D}}{3} a^{2}\right) \Sigma}\left[\left(r^{2}+a^{2}\right) \Delta_{\theta}-\Delta_{r}\right] \operatorname{sen}^{2} \theta \dot{\varphi} \\
p_{r} & =g_{r r} \dot{r} \\
& =\frac{\Sigma}{\Delta_{r}} \dot{r} \\
p_{\theta} & =g_{\theta \theta} \dot{\theta} \\
& =\frac{\Sigma}{\Delta_{\theta}} \dot{\theta} \\
p_{\varphi} & =g_{\varphi \varphi} \dot{\varphi}+g_{\varphi t} \dot{t} \\
& =\frac{1}{\left(1+\frac{\Lambda_{4 D}}{3} a^{2}\right)^{2} \Sigma}\left[\left(r^{2}+a^{2}\right)^{2} \Delta_{\theta}-\Delta_{r} a^{2} \operatorname{sen}^{2} \theta\right] \operatorname{sen}^{2} \theta \dot{\varphi}- \\
& -\frac{a}{\left(1+\frac{\Lambda_{4 D}}{3} a^{2}\right) \Sigma}\left[\left(r^{2}+a^{2}\right) \Delta_{\theta}-\Delta_{r}\right] \operatorname{sen}^{2} \theta \dot{t} .
\end{aligned}
$$

Tais momentos, com o uso de (4.85) e da métrica invertida

$$
g^{\mu \nu}=\left(\begin{array}{cccc}
-\frac{\left(r^{2}+a^{2}\right)^{2} \Delta_{\theta}-\Delta_{r} a^{2} \operatorname{sen}^{2} \theta}{\Sigma \Delta_{\theta} \Delta_{r}} & 0 & 0 & -\left(1+\frac{\Lambda_{4 D}}{3} a^{2}\right) \frac{\left(r^{2}+a^{2}\right)^{2} \Delta_{\theta}-\Delta_{r}}{\Sigma \Delta_{\theta} \Delta_{r}} \\
0 & \frac{\Delta_{r}}{\Sigma} & 0 & 0 \\
0 & 0 & \frac{\Delta_{\theta}}{\Sigma} & 0 \\
-\left(1+\frac{\Lambda_{4 D}}{3} a^{2}\right) \frac{\left(r^{2}+a^{2}\right)^{2} \Delta_{\theta}-\Delta_{r}}{\Sigma \Delta_{\theta} \Delta_{r}} & 0 & 0 & \left(1+\frac{\Lambda_{4 D}}{3} a^{2}\right)^{2}\left(\frac{\Delta_{r}-\Delta_{\theta} a^{2} \operatorname{sen}^{2} \theta}{\Sigma \Delta_{\theta} \Delta_{r}}\right) \operatorname{cosec}^{2} \theta
\end{array}\right)
$$

conduziram-nos à seguinte hamiltoniana:

\footnotetext{
${ }^{21}$ Usaremos, doravante, explicitamente a constante cosmológica, $\Lambda_{4 D}$, ao invés do raio $\mathrm{AdS} / \mathrm{dS}, L$, em todas expressões.
} 


$$
\begin{aligned}
H=\frac{1}{2 \Sigma}[- & \left(\frac{\left(r^{2}+a^{2}\right)^{2} \Delta_{\theta}-\Delta_{r} a^{2} \operatorname{sen}^{2} \theta}{\Delta_{\theta} \Delta_{r}}\right) p_{t}^{2}-2\left(1+\frac{\Lambda_{4 D}}{3} a^{2}\right)\left(\frac{\left(r^{2}+a^{2}\right)^{2} \Delta_{\theta}-\Delta_{r}}{\Delta_{\theta} \Delta_{r}}\right) p_{t} p_{\varphi} \\
& \left.+\Delta_{r} p_{r}^{2}+\Delta_{\theta} p_{\theta}^{2}+\left(1+\frac{\Lambda_{4 D}}{3} a^{2}\right)^{2}\left(\frac{\Delta_{r}-\Delta_{\theta} a^{2} \operatorname{sen}^{2} \theta}{\Delta_{\theta} \Delta_{r}}\right) \operatorname{cosec}^{2} \theta p_{\varphi}^{2}\right] .
\end{aligned}
$$

Como foi dito acima, duas quantidades conservadas são imediatamente reconhecidas pela existência dos campos de Killing $\xi_{t}$ e $\xi_{\phi}$. Estas são

$$
p_{t}=-E \quad \text { e } \quad p_{\varphi}=\Phi
$$

a energia e o momento angular, respectivamente, de uma partícula que percorre uma geodésica. A terceira quantidade conservada é $\delta$ - segundo Bardeen et al. [70], esta é a massa de repouso de uma partícula que se move numa geodésica qualquer. Sem perda de generalidade, para fótons $\delta=0$ e para partículas massivas $\delta=1$.

Para chegar na quarta e última quantidade conservada, resolvemos a equação de HamiltonJacobi na gravitação, que a partir da hamiltoniana (4.85), tornou-se

$$
\frac{\partial S}{\partial \mu}=\frac{1}{2} g^{\mu \nu} \frac{\partial S}{\partial x^{\mu}} \frac{\partial S}{\partial x^{v}}
$$

onde $S$ é a função principal de Hamilton, que se relaciona com os momentos generalizados por

$$
p_{\mu} \equiv \frac{\partial S}{\partial x^{\mu}}
$$

Para resolvê-la, assumimos, como Carter, que suas variáveis podem ser separadas; então, buscamos uma solução do tipo

$$
S= \pm \frac{1}{2} \delta^{2} \mu-E t+\Phi \varphi+S_{\theta}(\theta)+S_{r}(r)
$$

Inserimos (4.95) na equação (4.93) para obter, com a inversa da métrica dada por (4.90),

$$
\begin{aligned}
\pm \delta^{2} & =-\left[\frac{\left(r^{2}+a^{2}\right)^{2} \Delta_{\theta}-\Delta_{r} a^{2} \operatorname{sen}^{2} \theta}{\Sigma \Delta_{\theta} \Delta_{r}}\right] E^{2}+2 a\left(1+\frac{\Lambda_{4 D}}{3} a^{2}\right)\left[\frac{\left(r^{2}+a^{2}\right) \Delta_{\theta}-\Delta_{r}}{\Sigma \Delta_{\theta} \Delta_{r}}\right] E \Phi \\
& +\frac{\Delta_{r}}{\Sigma}\left(\frac{d S_{r}}{d r}\right)^{2}+\frac{\Delta_{\theta}}{\Sigma}\left(\frac{d S_{\theta}}{d \theta}\right)^{2}+\left(1+\frac{\Lambda_{4 D}}{3} a^{2}\right)^{2}\left[\frac{\Delta_{r}-\Delta_{\theta} a^{2} \operatorname{sen}^{2} \theta}{\Sigma \Delta_{\theta} \Delta_{r}}\right] \operatorname{cosec}^{2} \theta \Phi^{2} .
\end{aligned}
$$

Separamos, nesta última equação, termos que envolvem $r$ e $\theta$. Com isso, chegamos à igualdade 


$$
\begin{aligned}
& \Delta_{\theta}\left(\frac{d S_{\theta}}{d \theta}\right)^{2}+\frac{1}{\Delta_{\theta}}\left[\operatorname{asen} \theta E-\left(1+\frac{\Lambda_{4 D}}{3} a^{2}\right) \operatorname{cosec} \theta \Phi\right]^{2} \mp \delta^{2} a^{2} \cos ^{2} \theta \\
& =-\Delta_{r}\left(\frac{d S_{r}}{d r}\right)^{2}+\frac{1}{\Delta_{r}}\left[\left(r^{2}+a^{2}\right) E-a\left(1+\frac{\Lambda_{4 D}}{3} a^{2}\right) \Phi\right]^{2} \pm \delta^{2} r^{2}
\end{aligned}
$$

Ambos os lados de (4.97) são iguais a uma constante de separação $K$, conhecida como constante de Carter - nos símbolos $\pm \mathrm{e} \mp$, ao lado de $\delta^{2}$, o sinal superior refere-se à métrica de Sitter e o inferior à anti-de Sitter. Assim, pudemos escrever (4.97) como duas equações desacopladas:

$$
\begin{aligned}
& \frac{d S_{r}}{d r}= \pm \frac{1}{\Delta_{r}}\left\{\left[\left(r^{2}+a^{2}\right) E-a\left(1+\frac{\Lambda_{4 D}}{3} a^{2}\right) \Phi\right]^{2}-\Delta_{r}\left( \pm \delta^{2} r^{2}+K\right)\right\}^{\frac{1}{2}} \\
& \frac{d S_{\theta}}{d \theta}= \pm \frac{1}{\Delta_{\theta}}\left\{-\left[a \operatorname{sen} \theta E-\left(1+\frac{\Lambda_{4 D}}{3} a^{2}\right) \operatorname{cosec} \theta \Phi\right]^{2}-\Delta_{\theta}\left( \pm \delta^{2} a^{2} \cos ^{2} \theta-K\right)\right\}^{\frac{1}{2}}
\end{aligned}
$$

Definimos, então,

$$
Q=\Delta_{\theta} K-\left[\left(1+\frac{\Lambda_{4 D}}{3} a^{2}\right) \Phi-a E\right]^{2}
$$

de tal forma que

$$
\frac{d S_{\theta}}{d \theta}= \pm \frac{\sqrt{\Theta}}{\Delta_{\theta}}
$$

onde

$$
\Theta=Q-\cos ^{2} \theta\left[a^{2}\left( \pm \Delta_{\theta} \delta^{2}-E^{2}\right)+\left(1+\frac{\Lambda_{4 D}}{3} a^{2}\right)^{2} \operatorname{cosec}^{2} \theta \Phi^{2}\right] .
$$

A constante $Q$ foi a quarta e última constante obtida - uma relação entre as constantes $E, \Phi$ e a constante de separação de variáveis $K$. Para que se tenha, nas métricas consideradas, as interessantes geodésicas ou órbitas, as equatoriais ${ }^{22}(\theta=\pi / 2), Q$ deve necessariamente ser nula. Por (4.88), vemos que órbitas inteiramente contidas no plano equatorial têm $p_{\theta}=0(\dot{\theta}=0)$, o que implica em $\partial S_{\theta} / \partial \theta=0$, pela definição dos momentos (4.94). Então, de acordo com (4.101), numa órbita equatorial $\Theta$ deve ser nula, o que impõe a condição de que $Q$ igualmente seja nula.

\footnotetext{
${ }^{22}$ Equações das geodésicas equatoriais foram usadas no capítulo 5, que utilizou as geometrias construídas neste capítulo para um estudo de ótica geométrica.
} 
Por fim, uma outra definição foi feita:

$$
P=\left(r^{2}+a^{2}\right) E-\left(1+\frac{\Lambda_{4 D}}{3} a^{2}\right) a \Phi
$$

que nos conduziu a uma forma mais resumida de (4.98). Isto é,

$$
\frac{d S_{r}}{d r}= \pm \frac{\sqrt{\mathbb{R}}}{\Delta_{r}}
$$

com

$$
\mathbb{R}=P^{2}-\Delta_{r}\left( \pm \delta^{2} r^{2}+K\right)
$$

Com o olhar de volta às expressões dos momentos generalizados (4.86)-(4.89), agora com o que temos nas mãos, (4.101) e (4.104), pudemos obter as equações das geodésicas ou, pelo menos, como as coordenadas $r$ e $\theta$ variam com a variação do parâmetro afim $\mu$. Ou seja, sabemos por (4.87) que $\dot{r}$ está relacionada com $p_{r}$. Mas como $p_{r}=d S_{r} / d r$, então

$$
\Sigma \dot{r}=\sqrt{\mathbb{R}}
$$

Da mesma forma, por (4.88), sabendo que $p_{\theta}=d S_{\theta} / d \theta$, chegamos à

$$
\Sigma \dot{\theta}=\sqrt{\Theta}
$$

Aqui, levamos em consideração somente o valor positivo das equações (4.101) e (4.104).

Para construir as dependências de $t$ e $\varphi$ com o parâmetro afim $\mu$, a tarefa é um pouco mais complicada. Usamos (4.86) e (4.89), escritas como um sistema de duas equações e duas incógnitas $(\dot{t}, \dot{\varphi})$ :

$$
\begin{aligned}
E & =-g_{t t} \dot{t}-g_{t \varphi} \dot{\varphi} \\
\Phi & =g_{\varphi \varphi} \dot{\varphi}+g_{\varphi t} \dot{t}
\end{aligned}
$$

Primeiro para $\dot{t}$ : multiplicamos (4.108) por $g_{\varphi \varphi}$ e (4.109) por $g_{t \varphi}$ e conseguimos

$$
\left[\left(g_{\varphi t}\right)^{2}-g_{\varphi \varphi} g_{t t}\right] \dot{t}=\Phi g_{t \varphi}+E g_{\varphi \varphi}
$$

Usando as métricas obtidas nas seções 4.3 e 4.4, chegamos à

$$
\Sigma \dot{t}=\frac{\left(r^{2}+a^{2}\right) P}{\Delta_{r}}-\frac{a}{\Delta_{\theta}}\left[a E \operatorname{sen}^{2} \theta-\left(1+\frac{\Lambda_{4 D}}{3} a^{2}\right) \Phi\right]
$$


Finalmente para $\dot{\varphi}$ : multiplicamos (4.108) por $g_{\varphi t}$ e (4.109) por $g_{t t}$ e conseguimos

$$
\left[g_{\varphi \varphi} g_{t t}-\left(g_{\varphi t}\right)^{2}\right] \dot{\varphi}=\Phi g_{t t}+E g_{\varphi t}
$$

Igualmente, com as métricas acima citadas, chegamos à

$$
\Sigma \dot{\varphi}=\frac{a P}{\Delta_{r}}-\frac{1}{\Delta_{\theta}}\left[a E-\left(1+\frac{\Lambda_{4 D}}{3} a^{2}\right) \operatorname{cosec}^{2} \theta \Phi\right] .
$$

No fim, esse caminho nos conduziu a equações que nos indicam como cada coordenada varia com a variação do parâmetro afim $\mu$. Essas derivadas primeiras podem ser elencadas lado a lado para uma melhor leitura e visualização:

$$
\begin{aligned}
\Sigma \dot{t} & =\frac{\left(r^{2}+a^{2}\right) P}{\Delta_{r}}-\frac{a}{\Delta_{\theta}}\left[a E \operatorname{sen}^{2} \theta-\left(1+\frac{\Lambda_{4 D}}{3} a^{2}\right) \Phi\right] \\
\Sigma \dot{r} & =\sqrt{\mathbb{R}} \\
\Sigma \dot{\theta} & =\sqrt{\Theta} \\
\Sigma \dot{\varphi} & =\frac{a P}{\Delta_{r}}-\frac{1}{\Delta_{\theta}}\left[a E-\left(1+\frac{\Lambda_{4 D}}{3} a^{2}\right) \operatorname{cosec}^{2} \theta \Phi\right] .
\end{aligned}
$$

Foi somente em 2010 que as equações das geodésicas das métricas Kerr-de Sitter e Kerranti-de Sitter foram resolvidas explicitamente - mais de quarenta anos depois do trabalho de Carter onde tais métrica foram criadas. Num exaustivo trabalho, Hackmann et al. [66] afirmam peremptoriamente tal feito. Esses autores resolveram, no contexto da relatividade geral, equações semelhantes às (4.114)-(4.117).

Como já dissemos, as equações (4.114)-(4.117) foram úteis no próximo capítulo, onde um estudo de ótica geométrica foi realizado com as soluções no contexto de mundos brana. 


\section{Capítulo 5}

\section{Ótica geométrica em espaços-tempo}

\section{curvos}

Neste capítulo, utilizamos o formalismo de Newman-Penrose para o cálculo de grandezas óticas — tais como a rotação do plano de polarização da luz e a variação do cisalhamento —, conforme Pineault e Roeder, em [37], que desenvolveram a abordagem, que aqui descrevemos e usamos, para a métrica de Kerr. Tal abordagem é valida somente quando a frequência da radiação é alta, o que equivale a um pequeno comprimento de onda. Neste caso, o tratamento é o da ótica geométrica, isto é, feixes de luz percorrem geodésicas do tipo nula, e o seus vetores de polarização propagam-se paralelamente ao longo da curva. Com essa abordagem, construímos grandezas óticas para as métricas com simetria axial e constante cosmológica, num contexto de mundos branas, estudadas e expostas no capítulo anterior.

\subsection{A abordagem de Pineault e Roeder}

No trabalho Applications of geometrical optics to the Kerr metric, Pineault e Roeder utilizaram o formalismo das tetradas nulas para analisar propriedades óticas na geometria de Kerr em baixas rotações. A abordagem geométrica mostra-se útil quando o comprimento de onda da radiação estudada é pequeno em relação às grandezas envolvidas, de acordo com [71], capítulo 22. Neste caso, um feixe de luz caminha ao longo de uma geodésica do tipo luz ou nula, e o seu vetor de polarização é propagado paralelamente ao longo da curva nula. Tal feixe comporta-se como uma onda plana num espaço-tempo de curvatura desprezível. Com uma tetrada igual àquela usada no apêndice $\mathrm{D}\left\{e_{a \mu}\right\}=\left(m_{\mu}, \bar{m}_{\mu}, l_{\mu}, k_{\mu}\right)$, adotada no formalismo de Newman-Penrose, a direção $k^{\mu}$ aqui é escolhida como tangente à congruência de geodésicas do tipo nula, ou seja, $k^{\mu}=d x^{\mu} / d \mu$, onde $\mu$ é um parâmetro afim. Então, a direção definida por $k^{\mu}$ deve permanecer fixa quando submetida a uma rotação nula (trans- 
formação de Lorentz) do tipo (D.15). Como pode ser visto pelos coeficientes de spin em (D.19), impor que a direção $k^{\mu}$ seja tangente à congruência nula implica que o coeficiente $\kappa$ seja igualmente nulo. Da mesma forma, devemos impor, por conveniência, que o restante da tetrada deva propagar-se paralelamente à congruência; com isso, outros dois coeficientes de spin tornaram-se nulos:

$$
\varepsilon=\pi=0 .
$$

Com as imposições acima, especialmente a escolha de $\mu$ como um parâmetro afim, a equação da geodésica nula foi escrita como

$$
\nabla_{v} k^{\mu} k^{v}=0
$$

Pudemos definir, então, três quantidades óticas, conhecidas como escalares óticos ${ }^{1}$ :

$$
\begin{aligned}
\theta & =\frac{1}{2} \nabla_{\mu} k^{\mu} \\
\omega & =\left(\frac{1}{2} \nabla_{[v} k_{\mu]} \nabla^{v} k^{\mu}\right)^{\frac{1}{2}} \\
|\sigma| & =\left(\frac{1}{2} \nabla_{(v} k_{\mu} \nabla^{v} k^{\mu}-\theta^{2}\right)^{\frac{1}{2}} .
\end{aligned}
$$

O escalar $\theta$ indica expansão, $\omega$ indica rotação, e o módulo de $\sigma$ - escalar definido em (D.19) — indica distorção ou cisalhamento de imagens formadas pela congruência nula. Estes escalares são independentes dos outros membros da tetrada $\left(m_{\mu}, \bar{m}_{\mu}, l_{\mu}\right)$. A quantidade $|\sigma|$ tem grande importância na classificação de espaços-tempo na relatividade geral. De acordo com o teorema de Goldberg-Sachs²:

Uma solução no vácuo é algebricamente especial se e somente se possui uma congruência nula sem cisalhamento.

Portanto, é de grande interesse saber como $\sigma$ varia na direção $k^{\mu}$, ou seja, como nesta direção o cisalhamento é menor ou maior ou simplesmente nulo; para isso, utiliza-se a notação (D.26) e a equação de Newman-Penrose (D.34), que para as imposições acima assume uma forma simplificada

$$
D \sigma=(\rho+\bar{\rho}) \sigma+\Psi_{0}
$$

onde $D$ é o operador derivada covariante na direção $k^{\mu}, \rho$ é um dos coeficientes de spin listados em (D.19), e $\Psi_{0}$, definido em (D.30). Em [37], Pineault e Roeder estimaram a

\footnotetext{
${ }^{1}$ Notações que usam parênteses ou colchetes no entorno de índices significam: $\nabla_{(\mu} k_{v)}=\frac{1}{2}\left(\nabla_{\mu} k_{v}+\nabla_{v} k_{\mu}\right)$ e $\nabla_{[\mu} k_{v]}=\frac{1}{2}\left(\nabla_{\mu} k_{v}-\nabla_{v} k_{\mu}\right)$.

${ }^{2}$ Cf. [68], especialmente a seção 7.6, onde generalizações desse teorema são mostradas.
} 
variação, na direção da congruência nula, do cisalhamento para o buraco negro de Kerr, somente em baixas rotações. Faremos o mesmo para métricas num contexto de mundos brana construídas no capítulo anterior. Mas antes de obter esse resultado, deve-se, de acordo com os autores citados, escrever todo o formalismo para os referenciais localmente não girantes, expostos no apêndice E, para que, por lidarmos com simetria axial, uma simplificação seja feita.

Segundo Pineault e Roeder, a propagação de $m^{\mu}$ na direção de $k^{\mu}$,

$$
D m^{\mu}=0
$$

sendo

$$
m^{\mu}=\frac{\sqrt{2}}{2}\left(a^{\mu}+i b^{\mu}\right)
$$

conduz ao conhecimento sobre a varição do vetor de polarização da luz na direção da congruência nula. Em ótica geométrica, a luz tem vetor de onda $k$ ortogonal ao vetor de polarização $f$; isto quer dizer que

$$
k^{\mu} k_{\mu}=0, \quad D k^{\mu}=0
$$

$\mathrm{e}$

$$
k^{\mu} f_{\mu}=0, \quad D f^{\mu}=0 .
$$

As relações (5.9) e (5.10) permitem identificar o vetor de onda com o vetor nulo do formalismo de Newman-Penrose $\left(k^{\mu}\right)$ e o vetor $a^{\mu}$ com o vetor de polarização $f^{\mu}$. Isto porque pode-se identificar o plano dado pelos vetores $k^{\mu} \mathrm{e} a^{\mu}$ com o plano de polarização, que é propagado paralelamente na direção $k^{\mu}$, isto é, esse plano permanece inalterado na direção da congruência nula e corrobora a interpretação de $a^{\mu}$ como vetor de polarização. Para construir uma expressão para o vetor $a^{\mu}$, a partir do vetor $m^{\mu}$, os autores utilizaram a tetrada nula localmente ortonormal ${ }^{3}\left\{e_{(a)}^{(\mu)}\right\}=\left\{r^{(\mu)}, \bar{r}^{(\mu)}, q^{(\mu)}, p^{(\mu)}\right\}$ que obedece as relações (D.3), que se encontram no apêndice $D$ dedicado ao formalismo de Newman-Penrose ${ }^{4}$ :

$$
\begin{aligned}
p^{(\mu)} & =\frac{1}{\sqrt{2}}(1,-1,0,0), & q^{(\mu)} & =\frac{1}{\sqrt{2}}(1,1,0,0), \\
r^{(\mu)} & =\frac{1}{\sqrt{2}}(0,0,1, i), & \bar{r}^{(\mu)} & =\frac{1}{\sqrt{2}}(0,0,1,-i) .
\end{aligned}
$$

\footnotetext{
${ }^{3}$ Para essa base, tal como no apêndice E, o índice da tetrada foi colocado entre parênteses. Além disso, para discerni-la, os índices tensoriais estão igualmente entre parênteses.

${ }^{4}$ Para subir e descer os índices tensoriais da base $\left\{e_{(a)}^{(\mu)}\right\}=\left\{r^{(\mu)}, \bar{r}^{(\mu)}, q^{(\mu)}, p^{(\mu)}\right\}$ usa-se $g_{\mu \nu}=\eta_{\mu \nu}=$ $\operatorname{diag}(-1,1,1,1)$.
} 
A partir de uma transformação do tipo

$$
L_{(v)}^{(\mu)}=\delta_{(v)}^{(\mu)}+\frac{\left(k^{(\mu)}-p^{(\mu)}\right)\left(k_{(v)}-p_{(v)}\right)}{k^{(\alpha)} p_{(\alpha)}},
$$

aplicada na tetrada (5.11), obtiveram uma base nula apropriada a descrever uma congruência nula, onde $k^{\mu}$ é o seu vetor tangente. O próximo passo foi aplicar esta última transformação em $r^{(\mu)}$ e, em seguida, utilizar a transformação nula $m^{(\mu)} \rightarrow m^{(\mu)}-\Gamma k^{(\mu)}$ para obter o vetor igualmente nulo

$$
m_{*}^{(\mu)}=r^{(\mu)}-\Gamma p^{(\mu)}=\frac{1}{\sqrt{2}}(-\Gamma, \Gamma, 1, i)
$$

onde $\Gamma=k^{(v)} r_{(v)} / k^{(\alpha)} p_{(\alpha)}$. Mais uma rotação nula foi realizada, $m_{+}^{(\mu)}=m_{*}^{(\mu)}+\beta k^{(\mu)}$, com a seguinte imposição: $m_{+}^{(\mu)} u_{(\mu)}=0$. Ou seja, a transformação

$$
m_{+}^{(\mu)}=m_{*}^{(\mu)}-\left(\frac{m_{*}^{(\mu)} u_{(v)}}{k^{(\alpha)} u_{(\alpha)}}\right) k^{(\mu)}
$$

onde $u^{(\mu)}$ é a quadrivelocidade de um observador qualquer projetada na base nula (5.11), produziu um vetor tipo espaço que não tem componente temporal nesse referencial (referencial ou base onde os índices estão entre parênteses) e é ortogonal à linha mundo de um observador qualquer. Com a imposição de que esse observador esteja em repouso em relação à tetrada $(5.11)$, ou seja, $u^{(\mu)}=(1,0,0,0)$, sabendo que $m_{+}^{(\mu)}=a_{+}^{(\mu)}+i b_{+}^{(\mu)}$, Pineault e Roeder escreveram este último vetor, $m_{+}{ }^{(\mu)}$, em componentes:

$$
\begin{aligned}
& a_{+}^{(\mu)}=\frac{1}{\sqrt{2}}\left(0,-\frac{k^{(2)}}{k^{(0)}}, 1-K\left(k^{(2)}\right)^{2}, K k^{(2)} k^{(3)}\right) \\
& b_{+}^{(\mu)}=\frac{1}{\sqrt{2}}\left(0,-\frac{k^{(3)}}{k^{(0)}},-K k^{(2)} k^{(3)}, 1-K\left(k^{(3)}\right)^{2}\right),
\end{aligned}
$$

onde $K=1 /\left[k^{(0)}\left(k^{(0)}+k^{(1)}\right)\right]$. Na próxima seção, a assunção acima de que o referencial esteja em repouso permitirá que o formalismo localmente não girante seja usado. Para métricas com simetria axial, será adotado esse formalismo, onde o observador gira com o sistema, no caso um buraco negro.

Para finalizar, impuseram mais uma rotação nula em $m_{+}^{(\mu)}$,

$$
m_{+}^{(\mu)} \rightarrow m^{(\mu)}=e^{-i \chi_{m_{+}}^{(\mu)}}
$$


de tal foma que $\varepsilon$ seja nulo e esteja de acordo com (5.1). Sabendo por (D.19) que $\varepsilon$ é

$$
\varepsilon \equiv \frac{1}{2}\left(\nabla_{v} m_{\mu} \bar{m}^{\mu} k^{v}\right)=\frac{1}{2} D m_{\mu} \bar{m}^{\mu}=\frac{1}{2} D m_{(\mu)} \bar{m}^{(\mu)},
$$

com a substituição de (5.16) na definição acima, temos

$$
\begin{aligned}
\varepsilon & =\frac{1}{2}\left[D\left(e^{-i \chi} m_{+(\mu)}\right)\right] \bar{m}^{(\mu)} \\
& =\frac{1}{2}\left[-i e^{-i \chi} D \chi m_{+(\mu)}+e^{-i \chi} D m_{+(\mu)}\right] \bar{m}^{(\mu)} .
\end{aligned}
$$

Como $\varepsilon=0$,

$$
i D \chi m_{+(\mu)}=D m_{+(\mu)} .
$$

Com a multiplicação de ambos os lados de (5.19) por $\bar{m}_{+}^{(\mu)}$ - conhecendo a propriedade desse vetor $\left(m_{\mu} \bar{m}^{\mu}=1\right)$, de acordo com (D.3), e que por (5.17) $2 \varepsilon_{+}=D m_{+(\mu)} \bar{m}_{+}{ }^{(\mu)}-$, chega-se na expressão para a variação de $\chi$ (ângulo usado na última transformação nula) na direção da congruência nula:

$$
D \chi=-2 i \varepsilon_{+} .
$$

Temos, finalmente, depois de todas rotações nulas, uma expressão para $m^{(\mu)}$ :

$$
m^{(\mu)}=\frac{\sqrt{2}}{2}\left(a^{(\mu)}+i b^{(\mu)}\right)=e^{-i \chi} m_{+}^{(\mu)} .
$$

O resultado último forneceu-nos, então, o vetor de polarização $a^{(\mu)}$ projetado na tetrada (5.11).

Outra grandeza de interesse, como veremos na próxima seção, expressa na base (5.11), é

$$
\Psi_{0}=R_{(\mu)(v)(\gamma)(\delta)} k^{(\mu)} m^{(v)} k^{(\gamma)} m^{(\delta)}
$$

pois determina a variação de $\sigma$ na direção da congruência nula, como pode ser visto na equação (5.6). Mas como $m^{(\mu)}$ é dado por (5.21), $\Psi_{0}$ assume a forma

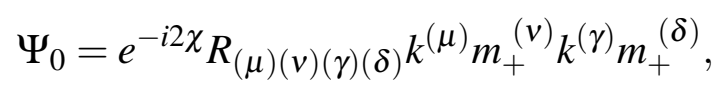

ou seja,

$$
\Psi_{0}=e^{-i 2 \chi} \Psi_{0+} .
$$

Para que tudo fique claro nos próximos passos, nesta seção foi exposta a abordagem de Pineault e Roeder que, com auxílio do formalismo de Newman-Penrose, expressa grandezas, 
como o vetor de polarização e o escalar $\Psi_{0}$, numa base ortonormal, indicada por vetores que possuem parênteses no entorno de seus índices tensoriais. Na seção seguinte, determinamos essa base ortonormal, fixamo-la com o auxílio da já exposta abordagem de Bardeen et al. no apêndice E, para que as grandezas acima mencionadas fossem calculadas.

\subsection{Aplicações às geometrias com simetria axial}

Nesta seção, utilizamos o formalismo exibido na seção anterior para determinar a variação do plano de polarização e a taxa de cisalhamento na direção $k^{\mu}$, escolhida como a direção da congruência nula, no caso das métricas com simetria axial num contexto de mundos brana expostas no capítulo 4. Para isso, construímos as componentes dos tensores de Riemann e conexão, projetadas numa base localmente não girante, úteis para o cálculo dessas grandezas. A base escolhida foi $\left\{e^{(a)}\right\}=\left\{e^{(\tau)}, e^{(r)}, e^{(\theta)}, e^{(\varphi)}\right\}$, dada por (E.3), com sua correspondente

dual escrita como (E.2). Como Pineault e Roeder, identificamos a base $\left\{e^{(a)}\right\}$ àquela escrita com seus índices tensoriais entre parênteses e projetamos as grandezas de interesse nela. Então, o problema físico é este: observador e fonte da luz estão em repouso em relação ao referencial $e^{(a)}$. Seja na posição da fonte ou do observador, o vetor de polarização é dado por (5.21).

Escrevemos as métricas construídas apresentadas no capítulo 4, (4.35) e (4.54), nas coordenadas $\tau, r, \theta$ e $\varphi$, na forma mais geral para o caso com simetria axial, como pode ser visto no apêndice $B$,

$$
d s^{2}=-e^{2 v} d \tau^{2}+e^{2 \psi}(d \varphi-\Omega d \tau)^{2}+e^{2 \lambda} d r^{2}+e^{2 \mu} d \theta^{2}
$$

$\operatorname{com} e^{2 \nu}, e^{2 \psi}, e^{2 \lambda}$ e $e^{2 \mu}$ dadas por:

$$
\begin{aligned}
e^{2 v}=\frac{\Sigma \Delta_{r} \Delta_{\theta}}{\Pi\left(1+\frac{\Lambda_{4 D}}{3} a^{2}\right)}, & e^{2 \psi}=\frac{\Xi \operatorname{sen}^{2} \theta}{\Sigma\left(1+\frac{\Lambda_{4 D}}{3} a^{2}\right)}, \\
e^{2 \lambda}=\frac{\Sigma}{\Delta_{r}}, & e^{2 \mu}=\frac{\Sigma}{\Delta_{\theta}},
\end{aligned}
$$

sendo

$$
\Pi=\frac{\left(r^{2}+a^{2}\right)^{2} \Delta_{\theta}-a^{2} \Delta_{r} \operatorname{sen}^{2} \theta}{\left(1+\frac{\Lambda_{4 D}}{3} a^{2}\right)}
$$

$\mathrm{e}$

$$
\Omega=-\frac{g_{\tau \varphi}}{g_{\varphi \varphi}}=\frac{a\left[\left(r^{2}+a^{2}\right) \Delta_{\theta}-\Delta_{r}\right]}{\Xi},
$$


onde $\Sigma=r^{2}+a^{2} \cos ^{2} \theta, \Delta_{r}=\left(r^{2}+a^{2}\right)\left(1-\Lambda_{4 D} r^{2} / 3\right)-2 M r+q, \Delta_{\theta}=1+\Lambda_{4 D} a^{2} / 3$, e $a$ é o parâmetro de rotação. A constante cosmológica na brana define o caso assintoticamente de Sitter, $\Lambda_{4 D}>0$, e o caso assintoticamente anti-de Sitter, $\Lambda_{4 D}<0$.

Como já vimos, um tensor qualquer pode ser projetado numa tetrada ortonormal de acordo com (E.6). O tensor de Riemann, por exemplo, na base não girante, usando a notação da seção anterior, escreve-se como:

$$
R_{(a)(b)(c)(d)}=R_{(\mu)(v)(\alpha)(\beta)} e_{(a)}^{(\mu)} e_{(b)}^{(v)} e_{(c)}^{(\alpha)} e_{(d)}^{(\beta)},
$$

com $a, b, c$ e $d$ fazendo o papel de índices da tetrada ortonormal; podem assumir valores de 1 a 4 , sendo que $\tau=0, r=1, \theta=2$ e $\varphi=3$. Portanto, as componentes dos tensor de Riemann e da conexão escritas no referencial localmente não girante são:

$$
\begin{aligned}
R_{(\tau)(\varphi)(\tau)(\varphi)} & =-R_{(r)(\theta)(r)(\theta)}=Q_{1}, \\
R_{(\tau)(\varphi)(r)(\theta)} & =-Q_{2}, \\
R_{(\tau)(r)(\tau)(r)} & =-\frac{1}{(1-z)}\left[Q_{1}(2+z)-\frac{q}{\Sigma^{2}}+\Lambda_{4 D}\right], \\
R_{(\varphi)(\theta)(\varphi)(\theta)} & =\frac{1}{(1-z)}\left[Q_{1}(2+z)+\frac{q}{\Sigma^{2}}+\Lambda_{4 D}\right], \\
R_{(\tau)(r)(\tau)(\theta)} & =R_{(\varphi)(r)(\varphi)(\theta)}=S Q_{2}, \\
R_{(\tau)(r)(\varphi)(r)} & =S\left[Q_{1}-\frac{q}{3 \Sigma^{2}}+\frac{\Lambda_{4 D}}{3}\right] \\
R_{(\tau)(\theta)(\varphi)(\theta)} & =-S\left[Q_{1}+\frac{q}{3 \Sigma^{2}}+\frac{\Lambda_{4 D}}{3}\right] \\
R_{(\tau)(r)(\varphi)(\theta)} & =-Q_{2} \frac{2+z}{1-z} \\
R_{(\tau)(\theta)(\tau)(\theta)} & =\frac{1}{(1-z)}\left[Q_{1}(1+2 z)+\left(\frac{q}{\Sigma^{2}}+\Lambda_{4 D}\right) z\right], \\
R_{(\varphi)(r)(\varphi)(r)} & =-\frac{1}{(1-z)}\left[Q_{1}(1+2 z)-\left(\frac{q}{\Sigma^{2}}-\Lambda_{4 D}\right) z\right], \\
R_{(\tau)(\theta)(\varphi)(r)} & =-Q_{2} \frac{1+2 z}{1-z}
\end{aligned}
$$

onde as funções $Q_{1}, Q_{2}, S$ e $z$ expressam-se por:

$$
\begin{aligned}
& Q_{1}=-\frac{\Lambda_{4 D}}{3}+\frac{\left[M r\left(r^{2}-3 a^{2} \cos ^{2} \theta\right)-q\left(r^{2}-a^{2} \cos ^{2} \theta\right)\right]}{\Sigma^{3}}, \\
& Q_{2}=-\frac{\Lambda_{4 D}}{3}+\frac{\left[M r\left(r^{2}-3 a^{2} \cos ^{2} \theta\right)-q\left(r^{2}-a^{2} \cos ^{2} \theta\right)\right]}{\Sigma^{3}},
\end{aligned}
$$




$$
\begin{aligned}
S & =\frac{3 a\left(r^{2}+a^{2}\right)\left(\Delta_{r} \Delta_{\theta}\right)^{1 / 2} \operatorname{sen} \theta}{\left(1+\frac{\Lambda_{4} D}{3} a^{2}\right) \Pi}, \\
z & =\frac{\Delta_{r} a^{2} \operatorname{sen}^{2} \theta}{\left(r^{2}+a^{2}\right)^{2} \Delta_{\theta}} .
\end{aligned}
$$

Da mesma forma, as componentes da conexão escritas nas base localmente não girante, projetadas nessa base, puderam ser escritas como:

$$
\Gamma_{(b)(c)}^{(a)}=\Gamma_{(\beta)(\gamma)}^{(\alpha)} e_{(\alpha)}^{(a)} e_{(b)}^{(\beta)} e_{(c)}^{(\gamma)} .
$$

Então, suas componentes não nulas nessa base são:

$$
\begin{aligned}
& \Gamma_{(r)(\tau)}^{(\tau)}=\Gamma_{(\tau)(\tau)}^{(r)}=\partial_{r} v e^{-\lambda} \\
& \Gamma_{(\theta)(\tau)}^{(\tau)}=\Gamma_{(\tau)(\tau)}^{(\theta)}=\partial_{\theta} v e^{-\mu}, \\
& \Gamma_{(\theta)(r)}^{(r)}=-\Gamma_{(r)(r)}^{(\theta)}=\partial_{\theta} \lambda e^{-\mu}, \\
& \Gamma_{(\theta)(\theta)}^{(r)}=-\Gamma_{(r)(\theta)}^{(\theta)}=-\partial_{r} \mu e^{-\lambda}, \\
& \Gamma_{(\varphi)(\varphi)}^{(r)}=-\Gamma_{(r)(\varphi)}^{(\varphi)}=-\partial_{r} \psi e^{-\lambda}, \\
& \Gamma_{(\varphi)(\varphi)}^{(\theta)}=-\Gamma_{(\theta)(\varphi)}^{(\varphi)}=-\partial_{\theta} \psi e^{-\mu} \\
& \Gamma_{(r)(\varphi)}^{(\tau)}=\Gamma_{(\tau)(\varphi)}^{(r)}=\Gamma_{(\varphi)(r)}^{(\tau)}=\Gamma_{(\varphi)(\tau)}^{(r)}=-\Gamma_{(\tau)(r)}^{(\varphi)}=-\Gamma_{(r)(\tau)}^{(\varphi)}=\frac{1}{2} \partial_{r} \Omega e^{\psi-\nu-\lambda}, \\
& \Gamma_{(\theta)(\varphi)}^{(\tau)}=\Gamma_{(\tau)(\varphi)}^{(\theta)}=\Gamma_{(\varphi)(\theta)}^{(\tau)}=\Gamma_{(\varphi)(\tau)}^{(\theta)}=-\Gamma_{(\tau)(\theta)}^{(\varphi)}=-\Gamma_{(\theta)(\tau)}^{(\varphi)}=\frac{1}{2} \partial_{\theta} \Omega e^{\psi-v-\mu} .
\end{aligned}
$$

As funções $v, \lambda, \mu$ e $\psi$ podem ser obtidas em (5.26). Todas as componentes não nulas desses tensores (Riemann e conexão) podem ser obtidas a partir das componentes acima escritas, utilizando-se simetrias associadas a esses dois tensores. Estas expressões reduzem-se às obtidas em [37] e [70] fazendo $q=0$ (a "carga de maré" $q$, como já discutimos no capítulo 4 , quantifica o efeito do bulk na brana) e $\Lambda_{4 D}=0$.

\subsubsection{Variação do vetor de polarização}

Desde os anos cinquenta do século passado, acredita-se que o campo gravitacional de um corpo em rotação altera, roda, o plano de polarização de ondas eletromagnéticas. Tal fenômeno, segundo [36], altera radicalmente a polarização de raios $\mathrm{X}$ provenientes de discos 
de acreção de buracos negros. Como Pineault e Roeder, Frederick et al. [38] estimaram a variação do vetor de polarização na geometria de Kerr. Os últimos utilizaram não somente os referenciais localmente não girantes, mas também usaram o chamado referencial global inercial, onde a direção da base de vetores permanece inalterada em relação às estrelas fixas.

De acordo com o formalismo de Pineault e Roeder, apresentado anteriormente, a variação do plano de polarização na direção da congruência nula, $k^{\mu}$, como já foi dito, é dada por $D \chi=-2 i \varepsilon_{+}$, conforme (5.20). Como o vetor de polarização é identificado com o $a^{(\mu)}$, sendo $m^{(\mu)}=\frac{\sqrt{2}}{2}\left(a^{(\mu)}+i b^{(\mu)}\right)=e^{-i \chi} m_{+}^{(\mu)}$, de acordo com (5.21), existe uma variação sua em relação ao vetor $a_{+}^{(\mu)}$, dada por uma rotação no sentido anti-horário expressa por um ângulo $\chi$. Para estimar quanto este ângulo varia na direção $k^{\mu}$, determinamos o coeficiente de spin $\varepsilon_{+}$, dado por $\varepsilon_{+}=\left(D m_{+(\mu)} \bar{m}_{+}^{(\mu)}\right) / 2$, que pôde ser escrito depois das transformações em $m^{\mu}$ (5.14), como:

$$
\varepsilon_{+}=\frac{1}{2}\left[\bar{m}_{(\mu)} D m^{(\mu)}+(\Delta-\bar{\Delta})\right], \quad \operatorname{com} \Delta=\bar{\Gamma} m_{(\mu)} D p^{(\mu)},
$$

onde, conforme (D.16), $D p^{(\mu)}=\Gamma_{(v)(\gamma)}^{(\mu)} p^{(v)} k^{(\gamma)}$. As componentes da conexão $\Gamma_{(v)(\gamma)}^{(\mu)}$, escritas na base $\left\{e^{(a)}\right\}=\left\{e^{(\tau)}, e^{(r)}, e^{(\theta)}, e^{(\varphi)}\right\}$, são aquelas expostas em (5.33). Assim, a variação do plano de polarização assume sua forma final, escrita em termos da conexão e do vetor $k^{\mu}$ projetados na base localmente não girante:

$$
\begin{aligned}
D \chi=-2 i \varepsilon_{+} & =\frac{\left(\Gamma_{(\theta)(\tau)}^{(\tau)} k^{(\tau)}+\Gamma_{(\theta)(r)}^{(r)} k^{(r)}+\Gamma_{(\theta)(\theta)}^{(r)} k^{(\theta)}+\Gamma_{(\theta)(\varphi)}^{(\tau)} k^{(\varphi)}\right) k^{(\varphi)}}{k^{(\tau)}+k^{(r)}} \\
& -\frac{\left(\Gamma_{(\varphi)(\tau)}^{(r)} k^{(\tau)}+\Gamma_{(\varphi)(r)}^{(\tau)} k^{(r)}+\Gamma_{(\varphi)(\theta)}^{(\tau)} k^{(\theta)}+\Gamma_{(\varphi)(\varphi)}^{(r)} k^{(\varphi)}\right) k^{(\theta)}}{k^{(\tau)}+k^{(r)}} \\
& +\Gamma_{(\varphi)(\varphi)}^{(\theta)} k^{(\varphi)}+\Gamma_{(\varphi)(\tau)}^{(\theta)} k^{(\tau)} .
\end{aligned}
$$

Para determinar a projeção de $k^{\mu}$ na base $e^{(a)}\left(k^{(\tau)}, k^{(r)}, k^{(\theta)}, k^{(\varphi)}\right)$ determinamos, primeiro, o vetor tangente à congruência e depois o projetamos na base (E.3). Como, já na seção 5.1, escolhemos $k^{\mu}=d x^{\mu} / d \mu-\operatorname{com} \mu$ representando um parâmetro afim —, usamos as equações geodésicas ${ }^{5}$ listadas no capítulo 4 (4.114)-(4.117) para determinar $k^{\mu}$, ou seja, $\dot{x}$ (como estamos lidando com um problema de ótica, o parâmetro $\delta$, nas equações geodésicas, é nulo). Dessa forma, para projetar $k^{\mu}$ na base localmente não girante, usamos (E.6) para

${ }^{5}$ Neste capítulo, usamos $\tau$ ao invés de $t$ para ficar de acordo com a métrica (5.25). 
chegar às expressões

$$
\begin{aligned}
k^{(0)}=k^{(\tau)} & =e^{v} k^{0}=e^{v} \dot{\tau}, \\
k^{(1)}=k^{(r)} & =e^{\lambda} k^{1}=e^{\lambda} \dot{r}, \\
k^{(2)}=k^{(\theta)} & =e^{\mu} k^{2}=e^{\mu} \dot{\theta}, \\
k^{(3)}=k^{(\varphi)} & =e^{\psi}\left(k^{3}-\Omega k^{1}\right)=e^{\psi}(\dot{\varphi}-\Omega \dot{\tau}),
\end{aligned}
$$

onde $e^{v}, e^{\lambda}, e^{\mu}, e^{\psi}$ e $\Omega$ foram listadas em (5.26) e (5.28), respectivamente.

Para um caso particular, quando o parâmetro $a$ nas métricas expostas no capítulo 4 é muito pequeno,

$$
D \chi=-\frac{\Phi \cos \theta}{r^{2} \operatorname{sen}^{2} \theta}+(3 M r-2 q) \frac{\sqrt{Q-\Phi^{2} \cot ^{2} \theta}}{r^{5}} a \operatorname{sen} \theta+\mathscr{O}\left(a^{2}\right)
$$

Nesse regime, a função definida em (4.102) tornou-se

$$
\Theta=Q-\Phi^{2} \cot ^{2} \theta
$$

$\mathrm{e}, \operatorname{por}(4.116), \operatorname{com} \Sigma \approx r^{2}$

$$
\sqrt{\Theta}=r^{2} \dot{\theta}=r^{2} \frac{d \theta}{d \mu} .
$$

As duas últimas simplificações, (5.38) e (5.39), quando substituídas em (5.37), forneceramnos

$$
D \chi=-\frac{\Phi \cos \theta}{r^{2} \operatorname{sen}^{2} \theta}+\left(3 M-\frac{2 q}{r}\right) \frac{a \operatorname{sen} \theta}{r^{2}} \frac{d \theta}{d \mu}+\mathscr{O}\left(a^{2}\right) .
$$

Como em todas grandezas de interesse que temos determinado (raio do horizonte de eventos, superfície de Killing), o parâmetro $q$ aparece. Como já discutimos no capítulo 4, a "carga de maré" $q$ mede a influência do bulk na brana. No resultado acima, a rotação do plano de polarização também depende desse parâmetro (curiosamente, não depende de $\Lambda_{4 D}$ diretamente). Com $q=0$, temos o mesmo resultado obtido por Pineault e Roeder em [37], embora $\Phi$ não seja o mesmo, pois nesta tese lidamos com geometrias assintoticamente de Sitter e anti-de Sitter (no trabalho desses autores, a geometria usada foi a de Kerr, que é assintoticamente plana). Quando o parâmetro de rotação é nulo, isto é, $a=0$, temos um curioso resultado: $D \chi$ não é nulo. Mesmo sabendo, por [37], que na geometria de Schwarzschild não existe rotação do plano de polarização, o resultado acima somente reafirma o que já foi dito sobre o deslocamento entre os vetores $a^{(\mu)}$ e $a_{+}^{(\mu)}$ - a expressão (5.40) mede somente o deslocamento entre os vetores $a^{(\mu)}$ e $a_{+}^{(\mu)}$. Portanto, em Schwarzschild o vetor de polarização, $a^{(\mu)}$, é constante ao longo de $k^{\mu}$ e está deslocado por um ângulo $\chi$ em relação a $a_{+}^{(\mu)}$, de acordo com (5.21) e 
com a definição $m^{(\mu)}=\sqrt{2}\left(a^{(\mu)}+i b^{(\mu)}\right) / 2$; o primeiro termo de (5.40) indica-nos isso. Por outro lado, quando $a \neq 0, \chi$ varia ao longo da congruência nula porque o segundo termo de (5.40) depende de $\dot{\theta}$, indicando-nos que a direção de $a^{(\mu)}$ varia em relação à direção de $a_{+}^{(\mu)}$.

Em mundos brana, o resultado que pode ser interpretado como ausência de rotação, quando o segundo termo de (5.40) é nulo e conduz à constância do vetor de polarização (mesmo quando $a \neq 0$ ), numa órbita com $r$ constante, pode ser obtido. Fazendo $r=2 q / 3 M$, o segundo termo de (5.40) é igualmente nulo. Mas para o regime que focamos, baixas rotações, $a \ll M$, esse ponto privilegiado fica dentro do horizonte de eventos. Por fim, órbitas equatoriais $(\theta=\pi / 2)$ conduzem ao seguinte resultado: $D \chi=0$ porque, como vimos no capítulo 4 na seção sobre geodésicas, $\Theta$ é nulo, o que implica em $\dot{\theta}=0$. Portanto, em trajetórias equatoriais a variação entre $a^{(\mu)}$ e $a_{+}^{(\mu)}$ é inexistente, seja qual for o caso (com ou sem rotação, na relatividade geral ou em mundos brana).

Mas para obter a variação do vetor de polarização, deve-se também computar o efeito do arrasto do espaço-tempo ${ }^{6}$. Tal variação de $a^{(\mu)}$, ou seja, $m^{(\mu)}$, segundo Pineault e Roeder, foi escrita como uma soma

$$
\Delta \omega=\Delta \chi+\Delta \varphi,
$$

onde o segundo termo à direita de (5.41) é a componente na variação de $a^{(\mu)}$ que surge devido ao arrasto do espaço-tempo. Para mostrar um caso específico, resolvemos primeiro (5.40). Para isso, uma nova coordenada $\psi$ foi introduzida para fazer o papel do ângulo azimutal no plano da órbita. Um novo ângulo, $\alpha$, que mede a inclinação do plano orbital em relação ao plano equatorial, foi relacionado com $\psi$ por

$$
\operatorname{sen} \alpha=\frac{\cos \theta}{\operatorname{sen} \psi} .
$$

Com a definição de $\alpha$, (5.40) tornou-se

$$
D \chi=-\frac{\Phi \cos \theta}{r^{2} \operatorname{sen}^{2} \theta}+D \chi^{\prime}+\mathscr{O}\left(a^{2}\right)
$$

onde

$$
D \chi^{\prime}=-\left(3 M-\frac{2 q}{r}\right) \frac{a \operatorname{sen} \alpha}{r^{2}} \frac{d(\operatorname{sen} \psi)}{d \mu} .
$$

A seguir, ilustramos dois casos de interesse.

\footnotetext{
${ }^{6}$ Como vimos, no capítulo 4, esse efeito - uma propriedade de geometrias com simetria axial — também foi notado no contexto de mundos brana adotado neste trabalho. Naquele capítulo, determinamos a ergosfera, que é resultado do arrasto, proveniente da rotação.
} 


\section{Congruência nula paralela ao eixo de simetria}

Continuamos com Pineault e Roeder e calculamos (5.41) para dois casos. No primeiro, o plano orbital faz um ângulo de $\pi / 2$ em relação ao plano equatorial, ou seja, $\alpha=\pi / 2$. Além disso, assumimos que a fonte está no plano equatorial e o observador num plano acima (ambos girando com o buraco negro), sendo a congruência nula paralela ao eixo de simetria, de acordo com a figura 5.1, a uma distância $d$ daquele eixo. Para um resultado válido no limite do campo fraco, assumimos a relação

$$
\operatorname{sen} \psi=\frac{\sqrt{r^{2}-d^{2}}}{r}
$$

que usada em (5.44), além de $\Phi=0$, pois não há momento angular para a partícula, conduziunos à

$$
D \chi^{\prime}=-\frac{a}{r^{2}}\left(3 M-\frac{2 q}{r}\right)\left(\frac{d^{2}}{r^{2}} \frac{1}{\sqrt{r^{2}-d^{2}}}\right) \frac{d r}{d \mu}
$$

ou seja,

$$
\int_{0}^{\chi} d \chi^{\prime}=-\int_{d}^{r_{o}}\left(\frac{3 M r-2 q}{r^{5}}\right) \frac{a d^{2}}{\sqrt{r^{2}-d^{2}}} d r
$$

pois $D \chi^{\prime}=k^{1} d \chi^{\prime} / d r$ e $k^{1}=d r / d \mu$. A integral acima resultou em

$$
\begin{aligned}
\Delta \chi & =-\left\{\sqrt{r_{o}^{2}-d^{2}}\left[\frac{M}{r_{o}}\left(\frac{1}{r_{o}^{2}}+\frac{2}{d^{2}}\right)-\frac{q}{2 r_{o}^{2}}\left(\frac{1}{r_{o}^{2}}+\frac{3}{2 d^{2}}\right)\right]\right. \\
& \left.-\frac{3 q}{4 d^{3}}\left(\frac{\pi}{2}-\arctan \left[\frac{d}{\sqrt{r_{o}^{2}-d^{2}}}\right]\right)\right\} a,
\end{aligned}
$$

sendo $r_{o}$ a posição do observador. Com $\Lambda_{4 D}=0$ e $r_{o} \rightarrow \infty$, temos

$$
\Delta \chi=-\left(\frac{2 M a}{d^{2}}-\frac{3 \pi a q}{8 d^{3}}\right)
$$

A expressão acima para $\Delta \chi$ é a mesma que Pineault e Roeder [37] obtiveram para a métrica de Kerr, desde que $q=0$. A influência do bulk na brana acentua a variação entre $a^{(\mu)}$ e $a_{+}^{(\mu)}$ quando assume valores negativos.

Para determinar $\Delta \varphi$ - a componente devido ao arrasto - , neste caso, usamos o conhecimento que o vetor de polarização, na fonte ou no observador, gira com o buraco negro. Isto é, sua velocidade de rotação, num referencial fixo, é dada por $\Omega$. Sendo assim, 


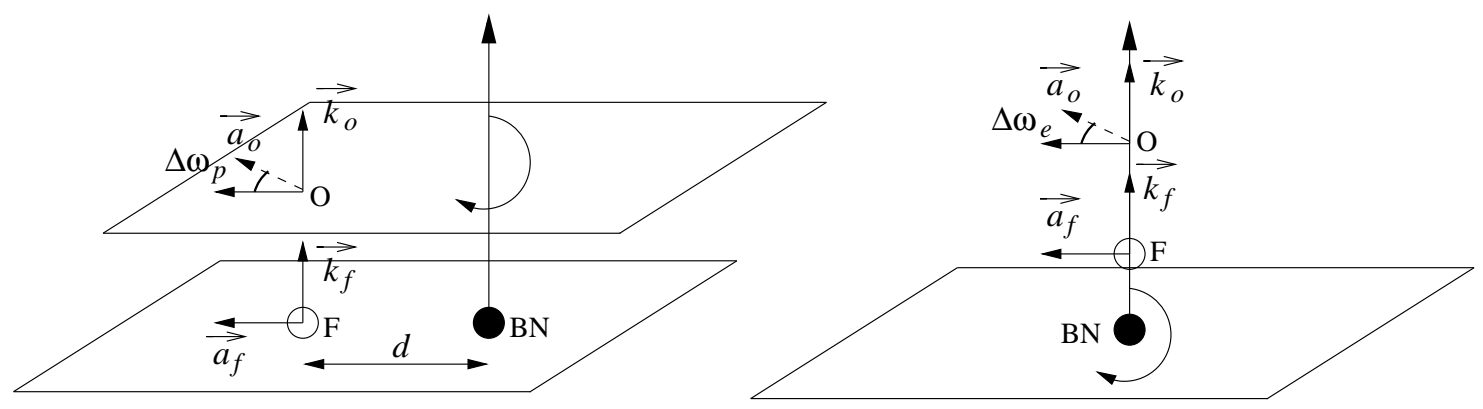

Figura 5.1: Variação do vetor de polarização com a rotação de um buraco negro. À esquerda, o caso onde a congruência nula propaga-se paralelamente ao eixo de rotação do buraco negro $(\mathrm{BN})$; fonte $(\mathrm{F})$ e observador (O) encontram-se a uma distância $d$ daquele eixo. À direita, o caso onde fonte e observador situam-se no eixo de simetria. Em ambas figuras, $\vec{k}$ e $\vec{a}$ representam o vetor de onda e o vetor de polarização, respectivamente. A variação de $\vec{a}$ é dada por $\Delta \omega_{p}$, à esquerda, e $\Delta \omega_{e}$, à direita.

$$
\begin{aligned}
d \varphi & =\Omega d t=\Omega\left(\frac{d t}{d r}\right) d r \\
& =\Omega \frac{k^{0}}{k^{1}} d r
\end{aligned}
$$

de acordo com (5.36). Para $a^{2} \ll M^{2}$, baixas rotações, temos, de acordo com (5.50),

$$
\begin{aligned}
\int_{\varphi_{i}}^{\varphi_{f}} d \varphi & =\int_{d}^{r_{0}}\left[\frac{2 M r-q}{r^{3}}+\frac{\Lambda_{4 D}}{3} r\right] \frac{a E}{\sqrt{E^{2} r^{2}-d^{2}}} d r \\
\Delta \varphi & =\left\{\left[\left(\frac{2 M}{d^{2}}-\frac{q}{2 d^{2} r_{o}}\right) E^{2}+\frac{\Lambda_{4 D}}{3} r_{0}\right] \sqrt{E^{2}-\frac{d^{2}}{r_{o}^{2}}-\left[\left(\frac{2 M}{d^{2}}-\frac{q}{2 d^{3}}\right) E^{2}\right.}\right. \\
& \left.\left.+\frac{\Lambda_{4 D}}{3} d\right] \sqrt{E^{2}-1}-\frac{q E^{4}}{2 d^{3}}\left(\arctan \left[\frac{1}{\sqrt{E^{2}-1}}\right]-\arctan \left[\frac{1}{\sqrt{\frac{E^{2} r_{o}^{2}}{d^{2}}-1}}\right]\right)\right\} \frac{a}{E} .
\end{aligned}
$$

Considerando o caso onde a brana não tem constante cosmológica (afim de compararmos com os resultados obtidos em Kerr), a energia da partícula, dada por (4.108), pôde ser considerada unitária no "infinito". Isto é

$$
\lim _{r \rightarrow \infty} E=1 .
$$

Além disso, adotamos $r_{o} \rightarrow \infty$, e (5.51) tornou-se:

$$
\Delta \varphi=\left(\frac{2 M}{d^{2}}-\frac{\pi q}{4 d^{3}}\right) a .
$$


Por fim, a variação do vetor de polarização (5.41), no exemplo onde não há constante cosmológica na brana, é

$$
\Delta \omega_{p}=\Delta \chi+\Delta \varphi=\frac{\pi a q}{8 d^{3}} .
$$

Quando $q=0$, o resultado de Pineault e Roeder [37] e Su e Mallett [38] é alcançado. Mais uma vez, no contexto de mundos brana que adotamos, temos uma situação, um exemplo, onde o valor de uma grandeza física não corresponde ao seu valor obtido no contexto da relatividade geral. $\mathrm{O}$ influência do bulk na brana fez-se notar novamente. $\mathrm{O}$ curioso aqui é que $\operatorname{com} q<0$, o vetor de polarização roda no sentido oposto ao da rotação do buraco negro.

\section{Congruência nula no eixo de simetria}

No segundo caso, fonte e observador estão no eixo de simetria, de acordo com a figura 5.1. Com isso, $\theta=0$, o que implicou em $D \chi^{\prime}=0$, conforme (5.44), e $D \chi=0$, pois $\Phi=0$. O procedimento, dessa vez, para obter-se a variação do vetor de polarização foi o mesmo do exemplo anterior. A diferença única foi assumir que $d=0$ na equação (5.51), tornando-a mais simples:

$$
\begin{aligned}
\int_{\varphi_{i}}^{\varphi_{f}} d \varphi & =\int_{r_{f}}^{r_{o}}\left[\frac{2 M r-q}{r^{4}}+\frac{\Lambda_{4 D}}{3}\right] a d r \\
\Delta \varphi & =\Delta \omega_{e}=\left[M\left(\frac{1}{r_{f}^{2}}-\frac{1}{r_{o}^{2}}\right)-\frac{q}{3}\left(\frac{1}{r_{f}^{3}}-\frac{1}{r_{o}^{3}}\right)-\frac{\Lambda_{4 D}}{3}\left(r_{f}-r_{o}\right)\right] a,
\end{aligned}
$$

sendo $r_{f}$, na integral definida, a posição da fonte. Com $\Lambda_{4 D}=0$ e $r_{o} \rightarrow \infty$, (5.55) apresentouse como

$$
\Delta \varphi=\Delta \omega_{e}=\left(\frac{M}{r_{f}^{2}}-\frac{q}{3 r_{f}^{3}}\right) a .
$$

Novamente, quando $q=0$, temos o resultado de Pineault e Roeder [37] e Godfrey [79], que usou uma outra abordagem. Em relação ao contexto da relatividade geral, comparando com a geometria de Kerr, (5.56) indica-nos uma maior variação do vetor de polarização quando $q<0$, sendo, mais uma vez, este o caso onde os efeitos gravitacionais do espaço-tempo 5-dimensional anti-de Sitter na brana são amplificados.

\subsubsection{Variação do cisalhamento na direção da congruência nula}

Como já foi dito no começo deste capítulo, determinamos a variação do cisalhamento $(\sigma)$ na direção da congruência nula, na direção $k^{\mu}$, no caso das métricas com simetria axial 
e constante cosmológica no cenário retratado no capítulo 4. Pela equação (5.6), a variação de $\sigma$ depende linearmente de $\Psi_{0}$. Então, faz-se necessário o cálculo deste último. Seguindo Pineault e Roeder, sabemos, por (5.23) e (5.24), que $\Psi_{0}=e^{-i 2 \chi} \Psi_{0+}$. O cálculo de $\Psi_{0+}$ mostrou-se, segundo os autores, tedioso e grande. Assim como no trabalho que usamos nesta tese como referência [37], apenas expusemos o resultado final dessa tarefa. Dessa forma, as partes real e imaginária de $\Psi_{0+}$ foram escritas como:

$$
\begin{aligned}
\operatorname{Re}\left(\Psi_{0+}\right) & =\frac{1}{2\left(k^{(\tau)}+k^{(r)}\right)^{2}}\left[-\zeta Q_{1}+\eta Q_{2}+2 S\left(Q_{2} \mathscr{G}+Q_{1} \mathscr{H}\right)\right] \\
\operatorname{Im}\left(\Psi_{0+}\right) & =\frac{1}{2\left(k^{(\tau)}+k^{(r)}\right)^{2}}\left[-\eta Q_{1}-\zeta Q_{2}+2 S\left(Q_{2} \mathscr{H}-Q_{1} \mathscr{G}\right)\right],
\end{aligned}
$$

onde

$$
\zeta=\mathscr{A}+c_{1} \mathscr{C}-c_{2} \mathscr{E}, \quad \eta=\mathscr{B}+c_{1} \mathscr{D}-c_{2} \mathscr{F}
$$

$\mathrm{e}$

$$
c_{1}=\frac{2+z}{1-w}, \quad c_{2}=\frac{1+2 z}{1-z} .
$$

As funções $Q_{1}, Q_{2}, S$ e $z$ foram definidas em (5.31), e as componentes $k^{(\tau)}$ e $k^{(r)}$ fazem parte da projeção de $k^{\mu}$ na tetrada girante. As demais funções escritas acima dependem dessa projeção. Isto é,

$$
\begin{aligned}
& \mathscr{A}=\left(k^{(\theta)^{2}}-k^{(\varphi)^{2}}\right)\left[\left(k^{(\theta)^{2}}-k^{(\varphi)^{2}}\right)+\left(k^{(\tau)}+k^{(r)}\right)^{2}\right]+2 k^{(\tau)} k^{(r)}\left(k^{(\tau)}+k^{(r)}\right)^{2} \\
& \mathscr{B}=2 k^{(\theta)} k^{(\varphi)}\left[\left(k^{(\theta)^{2}}-k^{(\varphi)^{2}}\right)+\left(k^{(\tau)}+k^{(r)}\right)^{2}\right] \\
& \mathscr{C}=2\left(k^{(\tau)}+k^{(r)}\right)^{2}\left(k^{(\theta)^{2}}-k^{(\varphi)^{2}}\right) \\
& \mathscr{D}=4 k^{(\theta)} k^{(\varphi)}\left(k^{(\tau)}+k^{(r)}\right)^{2} \\
& \mathscr{E}=\left(k^{(\theta)^{2}}-k^{(\varphi)^{2}}\right)\left[\left(k^{(\theta)^{2}}-k^{(\varphi)^{2}}\right)-\left(k^{(\tau)}+k^{(r)}\right)^{2}\right]+2 k^{(\tau)} k^{(r)}\left(k^{(\tau)}+k^{(r)}\right)^{2} \\
& \mathscr{F}=2 k^{(\theta)} k^{(\varphi)}\left[\left(k^{(\theta)^{2}}-k^{(\varphi)^{2}}\right)-\left(k^{(\tau)}+k^{(r)}\right)^{2}\right] \\
& \mathscr{G}=k^{(\theta)}\left(k^{(\tau)}+k^{(r)}\right)\left[\left(k^{(\theta)^{2}}-k^{(\varphi)^{2}}\right)-\left(k^{(\tau)}+k^{(r)}\right)^{2}-2 k^{(\varphi)^{2}}\right] \\
& \mathscr{H}=k^{(\varphi)}\left(k^{(\tau)}+k^{(r)}\right)\left[\left(k^{(\theta)^{2}}-k^{(\varphi)^{2}}\right)-\left(k^{(\tau)}+k^{(r)}\right)^{2}+2 k^{(\theta)^{2}}\right] .
\end{aligned}
$$

Para um caso particular, por exemplo, onde trajetórias permanecem no plano equatorial $\left(\theta=\pi / 2, k^{(\theta)}=0\right)$ e o parâmetro $a$ da métrica (5.25) é pequeno, a expressão para $\Psi_{0}$ tornou- 
se mais simples. Nesse caso, como vimos anteriormente, $D \chi=0$. Podemos, então, sem perda de generalidade, considerar $\chi$ uma constante nula. Isso conduz à igualdade: $\Psi_{0+}=\Psi_{0}$. Então, a parte imaginária de $\Psi_{0+}$ é nula. Temos, consequentemente, uma mais resumida expressão para o escalar $\Psi_{0}$ :

$$
\Psi_{0}=\frac{Q_{1}}{2\left(k^{(\tau)}+k^{(r)}\right)^{2}}\left(\mathscr{A}+c_{1} \mathscr{C}-c_{2} \mathscr{E}-2 S \mathscr{H}\right) .
$$

Com as expressões para $k^{(\mu)}$ - calculadas em (5.36), e substituídas em $\mathscr{A}, \mathscr{C}, \mathscr{E}$ e $\mathscr{H}-, Q_{1}$ e $S$, definidas em (5.31), e $c_{1}$ e $c_{2}$, definidas em (5.60), chegamos em

$$
\Psi_{0}=\frac{3 \Phi^{2}}{r^{5}}\left(M-\frac{q}{r}-\frac{\Lambda_{4 D}}{3} r^{3}\right)\left(1-\frac{2 a E}{\Phi}\right) .
$$

No caso $q=\Lambda_{4 D}=0$, temos o resultado de Pineault e Roeder. A influência do bulk na brana e a constante cosmológica desta fazem-se presentes nesse resultado. Como no trabalho desses autores, para um valor negativo de $\Phi$, uma quantidade conservada pela partícula ${ }^{7}$, temos um maior valor de $\Psi_{0}$. Isto equivale a dizer que para fótons em trajetórias equatoriais no sentido contrário ao da rotação, o efeito do cisalhamento é amplificado, de acordo com (5.6). Em outras palavras, a variação do cisalhamento, na direção da congruência nula $(D \sigma)$, é maior quando fótons percorrem uma trajetória no sentido oposto ao da rotação. Mas em mundos brana, esse efeito é ainda maior quando $q<0$, o que mostra mais uma vez a ampliação dos esfeitos gravitacionais quando o valor da carga induzida é negativo. Esse efeito é máximo quando o espaço-tempo é assintoticamente $\operatorname{AdS}\left(\Lambda_{4 D}<0\right)$, assumindo igualmente um valor negativo para $q$.

\footnotetext{
${ }^{7}$ Como vimos no capítulo 4 , $\Phi$ é uma quantidade conservada da partícula ao longo da geodésica; no caso presente, um fóton. Mais especificamente, $\Phi$ é o momento da partícula na direção do eixo $z$ de rotação ( $p_{\varphi}=$ $\left.L_{z}=\Phi\right)$. Em trajetórias no sentido oposto ao da rotação, $p_{\varphi}$ é negativo.
} 


\section{Capítulo 6}

\section{Comentários finais}

Os mundos brana apresentam-se como um contexto além da relatividade geral. Nos chamados modelos Randall-Sundrum [17, 18], nosso universo está imerso num espaço-tempo 5-dimensional anti-de Sitter, conhecido como bulk. Com um cenário tão exótico, uma nova física surgiu. Seja em física de partículas, cosmologia ou gravitação, os mundos branas trouxeram à luz novos fenômenos, novas visões.

Com tão interessante cenário, estudos que envolvem buracos negros e buracos de minhoca têm sido realizados. Logo depois da publicação dos modelos Randall-Sundrum, Chamblin et al. [25] construíram a chamada corda negra. Na brana, a métrica foi a mesma da solução de Schwarzschild, no bulk, uma métrica com constante cosmológica negativa que se apresentou instável. Numa outra abordagem que partiu do seminal trabalho de Shiromizu et al. [26], onde as equações do campo gravitacional foram projetadas na brana, Casadio et al. [27] e Bronnikov et al. [28] obtiveram métricas com simetria esférica em branas sem constante cosmológica. Em nosso Black holes and wormholes in AdS branes [29], generalizamos os resultados desses autores, impondo constante cosmológica negativa na brana, chegando, com isso, numa família de soluções diversificada, parametrizada por uma constante $C$, que para determinados valores assumidos $(C \geqslant 0)$ forneceu-nos buracos negros singulares e regulares e buracos de minhoca $(C<0)$ assintoticamente anti-de Sitter.

Nesse mesmo caminho, conseguimos, para uma brana com constante cosmológica positiva, no artigo Wormholes in de Sitter branes [30], métricas de buracos de minhoca transitáveis. Desde o trabalho de Morris e Thorne [10], em 1988, esses objetos ganharam destaque. No trabalho desses autores, dentro do contexto da relatividade geral, buracos de minhoca são transitáveis, ou seja, servem como ponte ou túnel entre distantes regiões do espaço-tempo, além de servirem como máquinas do tempo. Mas para existirem, necessitam de um campo de matéria que viole algumas condições de energia. Entretanto, desde 1998, a observação da expansão acelerada do universo é tida como consequência de um campo que possui essa 
característica. Em mundos brana, no entanto, também é possível construir métricas com tensores energia-momento que descrevem campos violadores das condições de energia. As soluções expostas no capítulo 3 desta tese cumprem este requisito.

Com um grau de dificuldade maior, a construção de espaços-tempo com simetria axial, que descrevem buracos negros em rotação, foi feita, por exemplo, em 2002 por Modgil et al. [31], numa generalização do trabalho de Chamblin et al. [25]. Seguindo o artigo de Dadhich et al. [32], que adotou simetria esférica, Aliev e Gümrükçüoglu [33] mostraram soluções com simetria axial geradas a partir do ansatz de Kerr-Schild. No capítulo 4 desta tese e em Rotating black holes in a Randall-Sundrum brane with a cosmological constant [35], generalizamos estes resultados, partindo do ansatz de Kerr-Schild-(A)-dS. Ou seja, adicionamos a constante cosmológica (negativa ou positiva) e chegamos a curiosos resultados como, já relatado em trabalhos sem a constante cosmológica, a existência de uma carga do tipo coulombiana na brana, mesmo sem existir no mundo quadridimensional um campo maxwelliano. Tal carga, como vimos, surge da influência do bulk na brana, amplifica os efeitos gravitacionais em nosso espaço-tempo quadridimensional quando assume valores negativos $(q<0)$, tornando o horizonte de eventos e a ergosfera, por exemplo, regiões maiores do que as mesmas encontradas na relatividade geral.

Por fim, realizamos um estudo de ótica geométrica nas métricas com simetria axial mostradas no capítulo 4. Ótica geométrica é uma abordagem suficiente quando o comprimento de onda da radiação eletromagnética é pequeno. Desde os anos cinquenta do século XX acredita-se que o campo gravitacional pode alterar o vetor de polarização da luz. Nos trabalhos [79, 37, 38], estimativas foram feitas para essa previsão. No capítulo 5, usamos a abordagem de Pineault e Roeder [37] para o cálculo da variação do cisalhamento e do vetor de polarização na direção da congruência nula (direção do feixe de luz). Esses autores realizaram um estudo no contexto da relatividade geral, mas em nosso trabalho o contexto foi o de mundos branas. Como era esperado, alguns resultados foram diferentes. No caso de uma fonte de luz (uma estrela) orbitando no plano equatorial em torno do buraco negro no contexto que trabalhamos, a variação do vetor de polarização da luz emitida e medida por um observador no "infinito" (quando a constante cosmológica na brana é nula) não foi a mesma do que aquela exposta por Pineault e Roeder. Em nosso caso, a variação não foi nula, mas $\Delta \omega_{p}=\pi a q / 8 d^{3}$, sendo que com $q<0$, o vetor de polarização roda no sentido oposto ao da rotação do buraco negro. Para o caso em que a fonte e o observador estão no eixo de simetria do buraco negro, o valor estimado também foi outro: $\Delta \omega_{e}=a\left(3 M r_{f}-q\right) / 3 r_{f}^{3}$ contra $\Delta \omega_{e}=M a / r_{f}^{2}$ dos autores citados. Em ambas situações estudadas e comparadas, a influência do bulk na brana, medida pela "carga de maré" $q$, fez-se notar e indicou-nos mais um possível teste para detectar vestígios indiretos que possam corroborar a realidade dos mundos brana. 


\section{Apêndice A}

\section{Equações do campo gravitacional numa 3-brana}

Neste apêndice, mostramos a dedução das equações do campo gravitacional em uma 3-brana - imersa num bulk 5-dimensional - com o auxílio das equações de Gauss e Codazzi, das equações de Einstein em 5 dimensões e das condições de junção de Israel, de acordo com o seminal trabalho The Einstein equations on the 3-brane world [26], de Shiromizu, Maeda e Sasaki. Para isso, os autores relacionaram o tensor de Einstein na brana com elementos no bulk. Em seguida, assumiram que todos os campos, exceto o gravitacional, estão confinados na brana, além da simetria $\mathbb{Z}_{2}$ no bulk. Vale a pena enfatizar que as equações na 3-brana reduzem-se às equações de Einstein da relatividade geral no limite de baixas energias. O resultado apresentado neste apêndice é de extrema importância para esta tese sua espinha dorsal.

Nosso universo, uma brana 4-dimensional, será indicada por $\mathscr{M}$ e terá uma métrica $h_{\mu v}$; no caso do espaço-tempo 5-dimensional, sua métrica foi indicada por $g_{A B}$. Ambos tensores relacionam-se por

$$
h_{A B}=g_{A B}-n_{A} n_{B} \quad \text { e } \quad h^{A B}=g^{A B}-n^{A} n^{B} .
$$

$\mathrm{O}$ vetor unitário $n^{A}$ é ortogonal à brana. Já o vetor $e_{\mu}^{A}$ é tangente à hipersuperfície quadridimensional ${ }^{1}$, projeta vetores na brana. Então, a métrica na brana é construída pela projeção de $h_{A B}$ na mesma. Ou seja,

$$
h_{\mu v}=e_{\mu}^{A} e_{v}^{B} h_{A B} .
$$

\footnotetext{
${ }^{1}$ Os índices em grego vão de 0 a 3; os latinos, referentes ao bulk, vão de 0 a 4 . Quantidades na brana foram indicadas pelo número 4, entre parênteses, escrito em seus cantos superiores à esquerda. Assim como quantidades no bulk levam o número 5.
} 
Portanto, com auxílio de (A.2) e da relação $e_{\mu}^{A} e_{A}{ }^{\nu}=\delta_{\mu}^{\nu}$, temos

$$
h_{\mu v}=g_{\mu v} \quad \text { e } \quad h^{\mu v}=g^{\mu v}
$$

Algumas propriedades foram úteis na obtenção das equações desejadas:

$$
h_{B}^{A}=g_{B}^{A}-n_{B} n^{A}, \quad n_{A} n^{A}=1 \text { e } e_{A}^{\mu} n^{A}=0 .
$$

As equações de Gauss e Codazzi [48], ponto de partida desta dedução, foram escritas como

$$
{ }^{(4)} R_{\beta \gamma \delta}^{\alpha}={ }^{(5)} R_{B C D}^{A} e_{A}^{\alpha} e_{\beta}^{B} e_{\gamma}^{C} q_{\delta}^{D}+K_{\gamma}^{\alpha} K_{\beta \delta}-K_{\delta}^{\alpha} K_{\beta \gamma}
$$

e

$$
D_{v} K_{\mu}{ }^{\nu}-D_{\mu} K={ }^{(5)} R_{A B} n^{A} e_{\mu}^{B}
$$

respectivamente, onde

$$
K_{\mu v}=e_{\mu}^{A} e_{v}^{B} \nabla_{A} n_{B}
$$

é a curvatura extrínseca de $\mathscr{M}, K_{\mu}^{\mu}=K$ é o traço de $K_{\mu \nu}$, e $D_{\mu}$ é o operador derivada covariante em relação à métrica $h_{\mu \nu}$. Contraindo (A.4) com relação aos índices $\alpha$ e $\gamma$, chegase à

$$
{ }^{(4)} R_{\beta \delta}={ }^{(5)} R_{B C D}^{A} h_{\lambda \alpha} h^{\lambda \gamma} e_{A}^{\alpha} e_{\beta}^{B} e_{\gamma}^{C} e_{\delta}^{D}+K K_{\beta \delta}-K_{\delta}^{\alpha} K_{\beta \alpha}
$$

Como, por (A.2),

$$
\begin{aligned}
h_{B}^{A} & =h^{A E} h_{E B}=e_{\mu}^{A} e_{v}^{E} e_{E}^{\sigma} e_{B}^{\rho} h^{\mu v} h_{\sigma \rho} \\
& =e_{\mu}^{A} e_{B}^{\rho} h^{\mu v} h_{v \rho}
\end{aligned}
$$

pois $e_{v}^{E} e_{E}^{\sigma}=\delta_{v}^{\sigma}$, substituindo (A.8), com as devidas trocas de índices, em (A.7), temos o resultado

$$
{ }^{(4)} R_{\mu v}={ }^{(5)} R_{A B} e_{\mu}^{A} e_{v}^{B}-{ }^{(5)} R_{B C D}^{A} n_{A} n^{C} e_{\mu}^{B} e_{v}^{D}+K K_{\mu v}-K_{v}^{\alpha} K_{\alpha \mu}
$$

Com o tensor de Ricci em 4 dimensões, pode-se obter o escalar de Ricci contraindo (A.9) com a métrica $h^{\mu v}$

$$
\begin{gathered}
{ }^{(4)} R=h^{\mu v(4)} R_{\mu v} \\
{ }^{(4)} R={ }^{(5)} R_{A B} h^{A B}-h^{\mu \nu{ }^{(5)}} R_{B C D}^{A} n_{A} n^{C} e_{\mu}^{B} e_{v}^{D}+K^{2}-K^{\mu \alpha} K_{\mu \alpha} .
\end{gathered}
$$

Então, com auxílio de (A.9) e (A.10), as equações do campo gravitacional na brana tornaram- 
se, após alguma manipulação algébrica,

$$
\begin{aligned}
{ }^{(4)} G_{\mu v} & ={ }^{(4)} R_{\mu v}-\frac{1}{2} h_{\mu v}{ }^{(4)} R \\
& ={ }^{(5)} R_{A B} e_{\mu}^{A} e_{v}^{B}{ }^{(5)} R_{A B} h^{A B} \frac{1}{2} h_{\mu v}-\tilde{E}_{\mu v}+K K_{\mu v}-K_{v}^{\alpha} K_{\mu \alpha} \\
& -\frac{1}{2} h_{\mu v}\left[K^{2}-K^{\mu \alpha} K_{\mu \alpha}-h^{\mu v}{ }^{(5)} R_{B C D}^{A} n_{A} n^{C} e_{\mu}^{B} e_{v}^{D}\right],
\end{aligned}
$$

onde

$$
\tilde{E}_{\mu v}={ }^{(5)} R_{B C D}^{A} n_{A} n^{C} e_{\mu}^{B} e_{v}^{D} .
$$

O segundo termo de (A.11), usando (A.1), pôde ser escrito da seguinte forma

$$
\begin{aligned}
\frac{1^{(5)} R_{A B} h^{A B} h_{\mu v}}{} & ={ }^{(5)} R_{A B}\left(g^{A B}-n^{A} n^{B}\right) \frac{h_{\mu v}}{2} \\
& =g_{A B}{ }^{(5)} R e_{\mu}^{A} e_{v}^{B}-\frac{h_{\mu \nu}}{2}{ }^{(5)} R_{A B} n^{A} n^{B},
\end{aligned}
$$

pois $h_{\mu v}=g_{A B} e_{\mu}^{A} e_{v}^{B}$, devido à relação de ortogonalidade: $n_{A} e_{\mu}^{A}=0$. Com isso, (A.11) foi expressa como

$$
\begin{aligned}
{ }^{(4)} G_{\mu v} & =\left({ }^{(5)} R_{A B}-\frac{1}{2} g_{A B}{ }^{(5)} R\right) e_{\mu}^{A} e_{v}^{B}+\frac{1}{2} h_{\mu v}{ }^{(5)} R_{A B} n^{A} n^{B}-\tilde{E}_{\mu v}+K K_{\mu v} \\
& -K_{v}^{\alpha} K_{\mu \alpha}-\frac{1}{2} h_{\mu v}\left[K^{2}-K^{\mu \alpha} K_{\mu \alpha}-h^{\mu v}{ }^{(5)} R_{B C D}^{A} n_{A} n^{B} e_{\mu}^{C} e_{v}^{D}\right] .
\end{aligned}
$$

Mas sabendo que ${ }^{(5)} G_{A B}={ }^{(5)} R_{A B}-\frac{1}{2} g_{A B}{ }^{(5)} R$, então

$$
\begin{aligned}
{ }^{(4)} G_{\mu v} & ={ }^{(5)} G_{A B} h_{\mu}^{A} h_{v}^{B}-\tilde{E}_{\mu \nu}+K K_{\mu \nu}-K_{v}^{\alpha} K_{\mu \alpha}+ \\
& +\frac{1}{2} h_{\mu v}\left[{ }^{(5)} R_{A B} n^{A} n^{B}-K^{2}+K^{\mu \alpha} K_{\mu \alpha}+{ }^{(5)} R_{B C D}^{A} n_{A} n^{C} h^{B D}\right] .
\end{aligned}
$$

Novamente, com auxílio de (A.1), realiza-se a seguinte simplificação:

$$
\begin{aligned}
{ }^{(5)} R_{B C D}^{A} n_{A} n^{C} h^{B D} & ={ }^{(5)} R_{B C D}^{A} n_{A} n^{C}\left(g^{B D}-n^{B} n^{D}\right) \\
& ={ }^{(5)} R_{A B} n^{A} n^{B},
\end{aligned}
$$

pois ${ }^{(5)} R_{A B C D} n^{A} n^{B} n^{C} n^{D}=0$ (multiplicação de um tensor simétrico por um antissimétrico). A igualdade acima conduziu à 


$$
\begin{aligned}
{ }^{(4)} G_{\mu \nu}= & { }^{(5)} G_{A B} e_{\mu}^{A} e_{v}^{B}-\tilde{E}_{\mu \nu}+K K_{\mu v}-K_{v}^{\alpha} K_{\mu \alpha}+ \\
& +h_{\mu v}{ }^{(5)} R_{A B} n^{A} n^{B}-\frac{1}{2} h_{\mu v}\left(K^{2}-K^{\mu \alpha} K_{\mu \alpha}\right) .
\end{aligned}
$$

Para relacionar ${ }^{(4)} G_{\mu \nu}$ com o tensor energia-momento na brana, as equações do campo gravitacional em 5 dimensões,

$$
{ }^{(5)} G_{A B}={ }^{(5)} R_{A B}-\frac{1}{2} g_{A B}{ }^{(5)} R=\kappa_{5}^{2}{ }^{(5)} T_{A B}
$$

(onde $\kappa_{5}^{2}$ é a constante gravitacional em 5 dimensões, e e ${ }^{(5)} T_{\delta \sigma}$ é o tensor energia-momento do bulk), assim como o tensor de Weyl em 5 dimensões

$$
\begin{aligned}
{ }^{(5)} C_{A B C D} & ={ }^{(5)} R_{A B C D}-\frac{1}{3}\left(g_{A C}{ }^{(5)} R_{B D}-g_{A D}{ }^{(5)} R_{C B}-g_{B C}{ }^{(5)} R_{D A}+g_{B D}{ }^{(5)} R_{C A}\right) \\
& +\frac{1}{12}\left(g_{A C} g_{D B}-g_{A D} g_{C B}\right){ }^{(5)} R
\end{aligned}
$$

foram usados. A partir de (A.18), com auxílio de $g^{A B}$, o escalar de curvatura e o tensor de Ricci, em 5 dimensões, tomaram a seguinte forma

$$
{ }^{(5)} R=-\frac{2}{3} \kappa_{5}^{2}{ }^{(5)} T \quad \text { e } \quad{ }^{(5)} R_{A B}=\frac{\kappa_{5}^{2}}{3}\left(3^{(5)} T_{A B}-{ }^{(5)} T g_{A B}\right) .
$$

Substituindo-os na equação (A.17), chega-se à igualdade

$$
\begin{aligned}
{ }^{(4)} G_{\mu v} & =\kappa_{5}^{2}{ }^{(5)} T_{A B} e_{\mu}^{A} e_{v}^{B}-\tilde{E}_{\mu v}+K K_{\mu v}-K_{v}{ }^{\alpha} K_{\mu \alpha}+ \\
& +h_{\mu v} \frac{\kappa_{5}^{2}}{3}\left(3^{(5)} T_{A B}-{ }^{(5)} T g_{A B}\right) n^{A} n^{B}-\frac{1}{2} h_{\mu v}\left(K^{2}-K^{\mu \alpha} K_{\mu \alpha}\right) .
\end{aligned}
$$

Multiplicando (A.19) por $n^{A} n^{C} h_{\mu}^{B} h_{v}^{D}$ - com o auxílio de (A.3) -, (A.12) foi expressa como

$$
\begin{aligned}
\tilde{E}_{\mu \nu} & =\frac{1}{3}\left({ }^{(5)} R_{A B} e_{\mu}^{A} e_{v}^{B}{ }^{\left({ }^{(5)}\right.} R_{C D} n^{C} n^{D} g_{A B} e_{\mu}^{A} e_{v}^{B}\right)-\frac{1}{12}\left({ }^{(5)} R g_{A B} e_{\mu}^{A} e_{v}^{B}\right)+ \\
& +{ }^{(5)} C_{A B C D} n^{A} n^{C} e_{\mu}^{B} e_{v}^{D} .
\end{aligned}
$$

Com ajuda de (A.20), $\tilde{E}_{\mu \nu}$ finalmente assumiu a forma

$$
\tilde{E}_{\mu \nu}=\frac{\kappa_{5}^{2}}{3}{ }^{(5)} T_{A B} e_{\mu}^{B} e_{v}^{A}-\frac{1}{6} \kappa_{5}^{2}{ }^{(5)} T g_{A B} e_{\mu}^{B} e_{\nu}^{A}+\frac{1}{3} \kappa_{5}^{2}{ }^{(5)} T_{C D} n^{C} n^{D} h_{\mu \nu}+E_{\mu \nu},
$$

onde

$$
E_{\mu v}=C_{A B C D} n^{A} n^{C} e_{\mu}^{B} e_{v}^{D} .
$$


Uma propriedade de $E_{\mu \nu}$ é ter traço nulo, ou seja, $E_{\mu}^{\mu}=0$. Substituindo (A.22) em (A.21), temos as equações do campo gravitacional quadridimensionais em função do tensor energiamomento 5-dimensional, ou seja,

$$
\begin{aligned}
{ }^{(4)} G_{\mu v} & =\frac{2 \kappa_{5}^{2}}{3}\left({ }^{(5)} T_{A B} e_{\mu}^{A} e_{v}^{B}+\left[{ }^{(5)} T_{A B} n^{A} n^{B}-\frac{{ }^{(5)} T}{4}\right] h_{\mu v}\right)-E_{\mu v}+ \\
& +K K_{\mu v}-K_{v}^{\alpha} K_{\mu \alpha}-\frac{1}{2} h_{\mu v}\left(K^{2}-K^{\mu \alpha} K_{\mu \alpha}\right) .
\end{aligned}
$$

A expressão (A.24) nos revela que as equações do campo gravitacional em 4 dimensões, nesse cenário, possuem termos que não dependem do tensor energia-momento. Portanto, tais termos fazem o papel de uma matéria geométrica que curva o espaço-tempo, sendo a manifestação do bulk na brana.

A partir da equação (A.5), com o auxílio de (A.20) e do fato de que $h_{\mu v} n^{v}=0$, constrói-se a relação

$$
\begin{aligned}
D_{v} K_{\mu}{ }^{\nu}-D_{\mu} K & =\frac{\kappa_{5}^{2}}{3}\left(3^{(5)} T_{A B \sigma}-{ }^{(5)} T g_{A B}\right) n^{A} e_{\mu}^{B} \\
& =\kappa_{5}^{2}{ }^{(5)} T_{A B} n^{A} e_{\mu}^{B} .
\end{aligned}
$$

Até o presente momento, não foi utilizada qualquer tipo de simetria em relação ao tensor energia-momento. Então, usa-se, por exemplo, um cenário brana para lançar mão de algumas escolhas. Com a escolha de uma coordenada $y$, a coordenada da dimensão extra, adota-se uma única brana, localizada em $y=0$. Assim, $n_{\mu} d x^{\mu}=d y$, e a métrica do bulk pôde ser escrita como

$$
d s^{2}=h_{\mu \nu} d x^{\mu} d x^{v}+d y^{2} .
$$

Assumi-se também que o tensor energia-momento no bulk tem a forma

$$
{ }^{(5)} T_{A B}=-\Lambda_{5 D} g_{A B}+S_{A B} \delta(y),
$$

onde

$$
S_{\mu \nu}=e_{\mu}^{A} e_{\nu}^{B} S_{A B}=-\lambda h_{\mu v}+\tau_{\mu \nu},
$$

sendo $\Lambda_{5 D}$ a constante cosmológica em 5 dimensões, $\lambda$ e $\tau_{\mu \nu}$ a tensão e o tensor energiamomento, respectivamente, na brana e $\tau_{\mu v} n^{v}=0$. O tensor energia-momento $T_{A B}$, de acordo com (A.27), tem um comportamento singular devido à brana, que divide o bulk em duas partes, quebrando a simetria de translação em $y$. Assim, existe uma região de transição entre o bulk e a brana, que requer uma solução de continuidade dada pelas condições de junção de 
Israel [82]:

$$
\left[h_{\mu v}\right]=0
$$

$\mathrm{e}$

$$
\left[K_{\mu v}\right]=-\kappa_{5}^{2}\left(S_{\mu v}-\frac{1}{3} h_{\mu v} S\right),
$$

onde o operador $\left[X_{\mu v}\right]$ é definido como

$$
\left[X_{\mu v}\right]=\lim _{y \rightarrow+0} X-\lim _{y \rightarrow-0} X=X^{+}-X^{-} .
$$

Como nos modelos Randall-Sundrum, usa-se a simetria $\mathbb{Z}_{2}$ e fixa-se a brana num ponto fixo da dimensão extra. Com isso, a simetria imposta relaciona a curvatura extrínseca da brana com o seu tensor energia-momento

$$
K_{\mu \nu}^{+}=-K_{\mu v}^{-}=\frac{1}{2} \kappa_{5}\left(S_{\mu v}-\frac{1}{3} h_{\mu v} S\right) .
$$

Por causa dessa simetria, pode-se calcular quantidades em ambos os lados, + e -, da brana. Por fim, substituindo (A.32) em (A.24), conclui-se que as equações do campo gravitacional induzido na brana são:

$$
{ }^{(4)} G_{\mu v}=-\Lambda_{4 D} h_{\mu v}+8 \pi G_{N} \tau_{\mu v}+\kappa_{5}^{2} \pi_{\mu v}-E_{\mu v}
$$

onde

$$
\begin{gathered}
\Lambda_{4 D}=\frac{1}{2} \kappa_{5}^{2}\left(\Lambda_{5 D}+\frac{1}{6} \kappa_{5}^{2} \lambda^{2}\right), \\
G_{N}=\frac{\kappa_{5}^{2} \lambda}{48 \pi}
\end{gathered}
$$

$\mathrm{e}$

$$
\pi_{\mu \nu}=-\frac{1}{4} \tau_{\mu \alpha} \tau_{\nu}^{\alpha}+\frac{1}{12} \tau \tau_{\mu \nu}+\frac{1}{8} h_{\mu \nu} \tau_{\alpha \beta} \tau^{\alpha \beta}-\frac{1}{24} h_{\mu v} \tau^{2} .
$$

As equações (A.33) são, finalmente, as equações do campo gravitacional projetadas na brana e se tornam as conhecidas equações da relatividade geral quando se toma o limite de $\kappa \rightarrow 0$, mantendo $G_{N}$ finito e desprezando $E_{\mu \nu}$. O tensor $E_{\mu \nu}$ é a parte do tensor 5dimensional de Weyl projetado na brana e carrega consigo a informação do campo gravitacional fora dela, como o bulk a influencia. Portanto, $E_{\mu \nu}$ depende da geometria do bulk e não pode ser determinado livremente, além disso, porque é vinculado à matéria na brana. $\mathrm{Ou}$ 
seja, usando a identidade de Bianchi, $D^{\mu(4)} G_{\mu \nu}=0$, observa-se a relação entre $E_{\mu \nu}$ e $\tau_{\mu \nu}$

$$
\begin{aligned}
D^{\mu} E_{\mu v} & =K^{\alpha \beta}\left(D_{v} K_{\alpha \beta}-D_{\beta} K_{v \alpha}\right) \\
& =\frac{1}{4} \kappa_{5}^{2}\left[\tau^{\alpha \beta}\left(D_{v} \tau_{\alpha \beta}-D_{\beta} \tau_{v \alpha}\right)+\frac{1}{3}\left(\tau_{\mu v}-h_{\mu v} \tau\right) D^{\mu} \tau\right]
\end{aligned}
$$

Pode-se notar, então, que $E_{\mu v}$ não é livremente determinado, mas sua divergência é vinculada à matéria na brana. Com a decomposição de $E_{\mu \nu}$ em uma parte transversa, $E_{\mu \nu}^{T T}$, e numa parte longitudinal, $E_{\mu \nu}^{L}$, a última será determinada completamente pela matéria na brana. Dessa forma, se $E_{\mu \nu}^{T T}$ for zero, as equações (A.33) tratarão somente de elementos contidos na brana pois a parte transversa corresponde a ondas gravitacionais no bulk. Então, para resolver completamente as equações na brana deve-se, em geral, resolver as equações no bulk. 



\section{Apêndice B}

\section{Métricas com simetria axial ou esférica}

Numa variedade $\mathscr{M}$, num dado ponto $p$, vetores são definidos utilizando os conceitos de espaço tangente, $T_{P}$, e espaço cotangente, $T_{P}^{* 1}$. Podemos, então, escrever vetores num espaço tangente como operadores derivada direcional, quando escritos na base das coordenadas; por exemplo, se escolhermos as coordenadas $\left(x^{0}, x^{1}, x^{2}, \ldots, x^{n}\right)$ para mapear uma variedade $n$-dimensional, teremos os vetores

$$
\frac{\partial}{\partial x^{0}}, \frac{\partial}{\partial x^{1}}, \frac{\partial}{\partial x^{2}}, \frac{\partial}{\partial x^{3}}, \ldots, \frac{\partial}{\partial x^{n}}
$$

como vetores da base das coordenadas num espaço tangente $T_{P}$, num dado ponto $p$, à variedade. Neste mesmo ponto, podemos construir uma base em $T_{P}^{*}$, cujos vetores na base das coordenadas são

$$
d x^{0}, d x^{1}, d x^{2}, d x^{3}, \ldots, d x^{n}
$$

Bases tangente e cotangente obedecem a relação

$$
\omega_{a} e^{b}=\delta_{a}^{b}
$$

onde $e^{a}$ é uma base no espaço tangente e $\omega_{b}$ é uma base no espaço cotangente. ${ }^{2}$

Entre todos os campos vetoriais definidos numa variedade, os campos de Killing têm um papel importante na relatividade geral; utilizando-os, podemos fazer afirmações sobre simetrias numa variedade equipada com uma métrica lorentziana $\left(\mathscr{M}, g_{\mu v}\right)$ - chamaremos tal estrutura de espaço-tempo. Uma importante característica desses campos é que quando

\footnotetext{
${ }^{1}$ Cf. [49] e [50], especialmente o capítulo 2 de ambos.

${ }^{2}$ Os índices $a$ e $b$ vão de 0 a $n$.
} 
calculamos a derivada de Lie (£) da métrica $g_{\mu v}$ ao longo deles, esta anula-se. Ou seja,

$$
£_{\xi} g_{\mu \nu}=0
$$

onde $\xi$ é um campo de Killing. Esta simetria - a conservação da métrica sobre um campo de Killing — é uma isometria. A equação (B.4) é escrita explicitamente como

$$
\begin{aligned}
£_{\xi} g_{\mu v} & =\xi^{\alpha} \nabla_{\alpha} g_{\mu v}+\left(\nabla_{\mu} \xi^{\alpha}\right) g_{\alpha \nu}+\left(\nabla_{v} \xi^{\alpha}\right) g_{\mu \alpha} \\
& =\nabla_{\mu} \xi_{v}+\nabla_{v} \xi_{\mu}
\end{aligned}
$$

sendo $\nabla_{\mu}$ o operador derivada covariante. Conjugando os resultados (B.4) e (B.5), temos a conhecida equação de Killing ${ }^{3}$

$$
\nabla_{(\mu} \xi_{v)}=0
$$

Então, quando uma métrica é independente de uma coordenada temos um campo de Killing associado ao espaço-tempo descrito por essa métrica ou, ao contrário, quando um espaçotempo tem um campo de Killing, a métrica que o descreve é independente de uma coordenada.

Campos de Killing referem-se a grandezas conservadas, isto é, dada um geodésica $x^{\mu}(\lambda)$ com vetor tangente $v^{\mu}=d x^{\mu} / d \lambda$ e seja $\xi^{\mu}$ um campo de Killing, temos

$$
\begin{aligned}
v^{\mu} \nabla_{\mu}\left(\xi_{v} v^{v}\right) & =v^{\mu} v^{v} \nabla_{\mu} \xi_{v}+\xi_{v} v^{\mu} \nabla_{\mu} v^{v} \\
& =0
\end{aligned}
$$

pois o segundo termo da equação acima anula-se pelo fato de que $x^{\mu}(\lambda)$ é uma geodésica $\left(v^{\mu} \nabla_{\mu} v^{v}=0\right)$, e o primeiro anula-se devido à equação de Killing. Consequentemente, a grandeza $\xi_{\mu} v^{\mu}$ é conservada ao longo da geodésica.

Com o conceito de campos de Killing em mente, vamos construir a forma geral da métrica de um espaço-tempo estacionário com simetria axial. Um espaço-tempo estacionário é aquele em que os termos da métrica que o descrevem são independentes de $t$, a coordenada temporal; dito de outra forma, um espaço-tempo estacionário possui um campo de Killing tipo tempo, $\xi_{t}=\partial / \partial t$, ou seja, existe uma isometria de $\mathscr{M}$ em $\mathscr{M}$ cujas órbitas são curvas do tipo tempo (os vetores de Killing tangentes a essas curvas são do tipo tempo). Por outro lado, um espaço-tempo com simetria axial é aquele em que os termos da métrica que o descrevem são independentes de $\phi$, a coordenada angular azimutal, ou seja, este espaço-tempo possui

\footnotetext{
${ }^{3}$ As notações que usam parênteses ou colchetes no entorno de índices significam: $\nabla_{(\mu} \xi_{v)}=$ $\frac{1}{2}\left(\nabla_{\mu} \xi_{v}+\nabla_{v} \xi_{\mu}\right)$ e $\nabla_{[\mu} \xi_{v]}=\frac{1}{2}\left(\nabla_{\mu} \xi_{v}-\nabla_{v} \xi_{\mu}\right)$.
} 
um campo de Killing tipo espaço, $\xi_{\phi}=\partial / \partial \phi$, uma isometria de $\mathscr{M}$ em $\mathscr{M}$ cujas órbitas são curvas do tipo espaço fechadas. Então, um espaço-tempo estacionário com simetria axial é aquele que possui ambas isometrias, isto é, possui os campos de Killing $\xi_{t}$ e $\xi_{\phi}$ que comutam entre si. Ou seja,

$$
\left[\xi_{t}, \xi_{\phi}\right]=0 .
$$

Assim, uma solução estacionária com simetria axial é independente das coordenadas $t \mathrm{e}$ $\phi$ e pode ser escrita, genericamente num espaço-tempo 4-dimensional, por

$$
d s^{2}=\sum_{\mu, v} g_{\mu v}\left(x^{1}, x^{2}\right) d x^{\mu} d x^{v}
$$

onde $x^{0}=t$ e $x^{3}=\phi$, isto é, adotamos as coordenadas $\left(t, x^{1}, x^{2}, \phi\right)$ para representar grandezas físicas nesse espaço-tempo, sendo $x^{1}$ e $x^{2}$ coordenadas espaciais quaisquer. Como o tensor $g_{\mu \nu}$ é definido como um tensor simétrico, a métrica (B.9) terá 10 componentes indeterminadas que são funções de $x^{1}$ e $x^{2}$. Pode-se diminuir o número de componentes desconhecidas sabendo que a métrica deve ser invariante quando $t \rightarrow-t$ e $\phi \rightarrow-\phi$. Portanto, temos os termos nulos

$$
g_{01}=g_{02}=g_{31}=g_{32}=0 .
$$

Além disso, com a ajuda do teorema que mostra que uma métrica num plano - descrito pelas coordenadas $(x, y)$ e possuidor de uma métrica positiva ou negativa definida $(+,+$ ou,--$)$, escrita como

$$
d s^{2}=g_{x x} d x^{2}+2 g_{x y} d x d y+g_{y y} d y^{2}
$$

— pode ser expressa na forma diagonal

$$
d s^{2}= \pm e^{\Psi}\left(d x^{2}+d y^{2}\right),
$$

sendo $\Psi$ função de $x$ e $y$, conclui-se ${ }^{4}$ que a geometria (B.9), na forma geral para métricas com simetria axial, é

$$
d s^{2}=-e^{2 v} d t^{2}+e^{2 \psi}(d \varphi-\Omega d t)^{2}+e^{2 \lambda}\left(d x^{1}\right)^{2}+e^{2 \mu}\left(d x^{2}\right)^{2}
$$

onde $v, \psi, \Omega, \lambda$ e $\mu$ são funções de $x^{1}$ e $x^{2}$ apenas.

Um espaço-tempo é dito estático se ele pode ser folheado por uma família de hipersuperfícies ortogonais a um campo de Killing do tipo tempo ${ }^{5}$. Num espaço-tempo estático, há um

\footnotetext{
${ }^{4}$ Cf. [65], especialmente o capítulo 2.

${ }^{5}$ Cf. [50], p. 203 ou [67], p. 184.
} 
sistema de coordenadas adaptado ao campo de Killing do tipo tempo de tal forma que a métrica é independente da coordenada temporal $t$ e não possui termos cruzados do tipo $g_{t \mu}=0$. Por outro lado, um espaço-tempo 4-dimensional que possui simetria esférica tem três campos de Killing do tipo espaço linearmente independentes cujas órbitas são fechadas e satisfazem as relações de comutação abaixo:

$$
\begin{aligned}
& {\left[\xi_{1}, \xi_{2}\right]=\xi_{3},} \\
& {\left[\xi_{2}, \xi_{3}\right]=\xi_{1}}
\end{aligned}
$$

$\mathrm{e}$

$$
\left[\xi_{3}, \xi_{1}\right]=\xi_{2}
$$

Para um espaço-tempo estático com esta simetria, podemos escrever sua métrica 4-dimensional nas coordenadas $(t, r, \theta, \phi)$ na seguinte forma

$$
d s^{2}=-A(r) d t^{2}+\frac{1}{B(r)} d r^{2}+r^{2}\left(d \theta^{2}+\operatorname{sen}^{2} \theta d \phi^{2}\right),
$$

onde as funções $A(r)$ e $B(r)$ são dependentes unicamente da coordenada $r$. Um ponto importante a destacar é que todo espaço-tempo estacionário esfericamente simétrico é estático ${ }^{6}$.

Na relatividade geral, onde $A(r)=B(r)$ em (B.13), para o caso de vácuo, a mais geral solução é dada por

$$
A(r)=1-\frac{2 M}{r}+\frac{Q^{2}}{r^{2}}-\frac{\Lambda_{4 D}}{3} r^{2},
$$

que corresponde à métrica Reissner-Nordström-(A)-dS (AdS quando $\Lambda_{4 D}<0$ e dS quando $\left.\Lambda_{4 D}>0\right)$. Estas têm carga ${ }^{7} Q$ e constante cosmológica. Já a métrica de Reissner-Nordström tem somente carga, ou seja, $\Lambda_{4 D}=0$. As métricas de Schwarzschild-(A)-dS têm constante cosmológica e carga nula. Por fim, a métrica de Schwarzschild é aquela caracterizada pelo vácuo e ausência de carga e constante cosmológica $\left(Q=\Lambda_{4 D}=0\right)$.

\footnotetext{
${ }^{6}$ Cf. [49], cap. 6, p. 121.

${ }^{7}$ Carga nestas soluções pode ser elétrica e magnética. Ou seja, $Q$ pode ser escrita como a soma de ambas.
} 


\section{Apêndice C}

\section{Horizontes}

Podemos representar a estrutura causal de um espaço-tempo de forma muito útil através dos diagramas conforme ou diagramas de Penrose. Nestes, regiões infinitas podem ser representadas por linhas finitas e pontos. O infinito pode corresponder ao destino e/ou origem de curvas do tipo luz, tempo e espaço. Temos, então, três tipos de infinitos representados: tipo luz $\left(J^{+}, J^{-}\right)$, tipo tempo $\left(i^{+}, i^{-}\right)$e tipo espaço $\left(i^{0}\right)$, que podem corresponder ao destino e/ou origem de curvas do tipo luz, tempo e espaço, respectivamente. Exemplos ilustrativos são os diagramas para as geometrias de Minkowski e Schwarzschild, mostrados na figura C.1. No de Schwarzschild, exemplo (ii) na figura C.1, vemos que uma curva do tipo luz, um feixe de luz emitido por um observador A, não atinge o infinito $J^{+}$, quando dentro de uma região especial, delimitada por $r_{+}$. Por outro lado, no espaço-tempo de Minkowski, exemplo (i) na figura C.1, todas curvas do tipo luz terminam no infinito tipo luz $J^{+}$. Mas na geometria de Minkowski não há um buraco negro. Consequentemente, num espaço-tempo que contém um buraco negro, como no de Schwarzschild, existe uma região onde curvas do tipo luz ou do tipo tempo não terminam no infinito tipo luz $J^{+}$ou tipo tempo $i^{+}$; tais curvas terminam numa singularidade, em $r=0$. Assim, a fronteira dessa região especial, uma membrana de caminho único, é chamada de horizonte de eventos $\left(r_{+}\right.$indica o raio do horizonte de eventos, que é constante). Um observador depois que o atravessa jamais poderá sair, como é indicado na figura C.1. Então, para definir um horizonte de eventos, devemos conhecer a estrutura passada ou futura do infinito do tipo luz. Isto implica que um horizonte de eventos não seja um objeto local — ele é determinante na estrutura causal do espaço-tempo.

No entanto, a estrutura de infinitos em diagramas de Penrose de geometrias assintoticamente anti-de Sitter (AdS) ou de Sitter (dS) é um pouco diferente das acima citadas (Minkowski e Schwarzschild), assintoticamente planas. No caso de uma geometria assintoticamente AdS, como o espaço-tempo Schwarzschild-AdS, o exemplo (iii) na figura C.1, os infinitos do tipo luz, tempo e espaço são subsumidos pelo infinito $J$, uma superfície do tipo 
tempo. Para uma geometria assintoticamente dS, como a solução Schwarzschild-dS, o exemplo (iv) na figura C.1, todos os tipos de infinitos são representados por duas superfícies tipo espaço, uma no passado $J^{-}$e uma no futuro $J^{+}$. Para geometrias assintoticamente dS, temos um elemento a mais no diagrama de Penrose: o horizonte cosmológico, $r_{c}$. Tal como horizonte de eventos, o horizonte cosmológico é uma superfície nula, tipo luz. Na geometria de Sitter pura, ou seja, sem uma singularidade física, o único horizonte que existe é o horizonte cosmológico.
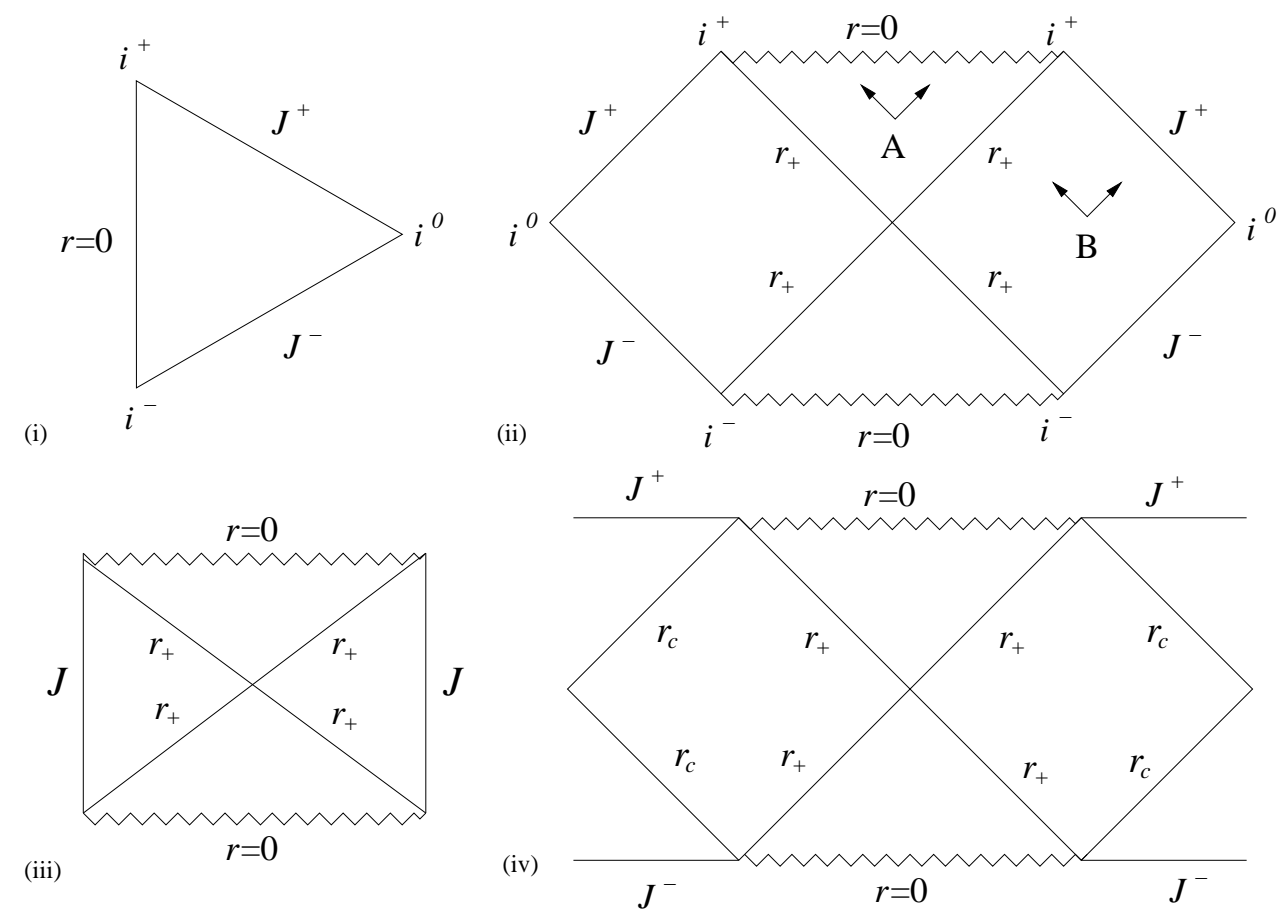

Figura C.1: Diagramas de Penrose dos espaços-tempo de Minkowski (i), Schwarzschild (ii), Schwarzschildanti de Sitter (iii) e Schwarzschild-de Sitter (iv). Em (ii), um observador $B$ pode enviar sinais de luz que "chegarão" no infinito $J^{+}$ou cruzarão o horizonte de eventos, $r=r_{+}$. Por outro lado, um observador $A$, dentro do horizonte, só poderá emitir sinais que terminarão na singularidade, $r=0$. Em Minkowski, entretanto, isso não ocorre; não há um horizonte de eventos. Nos diagramas (iii) e (iv) a estrutura de infinitos é um pouco diferente. Na geometria de Schwarzschild-AdS, todos infinitos são representados pela superfície tipo tempo $J$. Já para a métrica Schwarzschild-dS, os infinitos são representados, no passado, por $J^{-}$e, no futuro, por $J^{+}$, ambos indicados por uma superfície tipo espaço. Todos os diagramas foram retirados de [83].

Por outro lado, um horizonte de Killing é um objeto local, pois é definido como uma superfície $H$ que possui vetores de Killing $\xi^{\mu}$ tangentes do tipo luz, ou seja, $\left.\xi^{\mu} \xi_{\mu}\right|_{H}=0$ (a norma desse vetor especial, discutido no apêndice anterior, é nula em $H$ ). Em [14] é mostrado um expressivo resultado: num espaço-tempo estacionário e assintoticamente plano, o horizonte de eventos coincide com o horizonte de Killing, para algum campo de Killing $\chi^{\mu}$. 
Para obter a localização de um horizonte de eventos ou um horizonte cosmológico, devemos determinar as superfícies com vetores tangentes do tipo luz. Isto se deve porque um horizonte de eventos é uma superfície nula com raio constante ${ }^{1}$. Por ser de raio constante, um vetor ortogonal a essa superfície (igualmente nulo pois um vetor ortogonal a uma superfície nula é do mesmo modo nulo) pode ser escrito como o gradiente $\partial_{\mu} r$ ( $r$ é a superfície de raio constante), usando um sistema de coordenadas adequado do tipo $(t, r, \theta, \phi)$. A norma desse covetor é, então, dada por:

$$
\partial_{\mu} r \partial^{\mu} r=g^{\mu v} \partial_{v} r \partial_{\mu} r=g^{r r}
$$

sendo a coordenada $r$ independente das demais. Como já foi dito, a norma de $\partial_{\mu} r$ é nula, o que implica em

$$
\left.g^{r r}\right|_{\text {horizonte }}=0 .
$$

No caso de uma geometria ou métrica com simetria esférica, escrita na sua forma geral (B.13), $g^{\mu v}$ é

$$
g^{\mu \nu}=\left(\begin{array}{cccc}
-\frac{1}{A(r)} & 0 & 0 & 0 \\
0 & B(r) & 0 & 0 \\
0 & 0 & \frac{1}{r^{2}} & 0 \\
0 & 0 & 0 & \frac{\operatorname{cosec}^{2} \theta}{r^{2}}
\end{array}\right) .
$$

Portanto, a norma do covetor $\partial_{\mu} r$ é, por (C.1) e (C.2), dada pelos zeros de $B(r)$. Para a métrica Schwarzschild-de Sitter, cujo diagrama é o (iv) na figura C.1, a função $B(r)$ tem dois zeros positivos: o horizonte de eventos $\left(r_{+}\right)$e o horizonte cosmológico $\left(r_{c}\right)$.

No caso de uma métrica com simetria axial, expressa por (B.11) em sua forma geral, $g^{\mu \nu}$ é representado pela matriz

$$
g^{\mu v}=\left(\begin{array}{cccc}
-e^{-2 v} & 0 & 0 & -\Omega e^{-2 v} \\
0 & e^{-2 \lambda} & 0 & 0 \\
0 & 0 & e^{-2 \mu} & 0 \\
-\Omega e^{-2 v} & 0 & 0 & -\Omega^{2} e^{-2 v}+e^{-2 \psi}
\end{array}\right)
$$

Quando se passa a métrica axial em sua forma geral para as coordenadas de Boyer-Lindquist, como fizemos nos casos estudados no capítulo 4,

$$
g^{11}=e^{-2 \lambda}=g^{r r}=\frac{\Delta_{r}}{\Sigma}
$$

Portanto, os zeros da função $\Delta_{r}$ fornecem-nos os horizontes de eventos de uma métrica com simetria axial.

\footnotetext{
${ }^{1}$ Em [50], cap. 6, existem duas seções que tratam de horizontes de eventos e de Killing.
} 
Em caso de geometrias estáticas ou estacionárias, como visto no apêndice anterior, temos um campo de Killing imediato, dado pelo fato de que tais métricas são independentes da coordenada temporal $t$. Então, podemos construir o campo na base das coordenadas $(t, r, \theta, \phi)$ :

$$
\xi_{t}=\frac{\partial}{\partial t}
$$

Em coordenadas, $\xi_{t}$ pode ser escrito como

$$
\xi_{t}^{\mu}=(1,0,0,0)
$$

Então, a norma desse importante vetor é

$$
\xi_{t}^{2}=g_{\mu \nu} \xi_{t} \xi_{t}^{\mu}
$$

Nas geometrias que importam nesta tese, métricas estáticas ou estacionárias, a norma do campo de Killing $\xi_{t}$ sempre é dada por

$$
\xi_{t}^{2}=g_{t t}
$$

Segue que encontramos o horizonte de Killing para $\xi_{t}$, definido acima, fazendo $g_{t t}=0$.

Em geometrias estacionárias e não estáticas, geometrias com simetria axial, o horizonte de Killing, gerado por $\xi_{t}$, não coincide com o horizonte de eventos. Para que haja tal coincidência, é necessário construir o campo de Killing $\chi$, escrito como uma combinação dos campos $\xi_{t}$ e $\xi_{\phi}($ este último, de acordo com o apêndice B, define a simetria axial)

$$
\chi=\xi_{t}+\Omega_{+} \xi_{\phi}
$$

onde $\Omega_{+}$é a velocidade angular do buraco negro estimada no horizonte de eventos. Nele, no horizonte de eventos, temos

$$
\left.\chi^{2}\right|_{r=r_{+}}=0
$$




\section{Apêndice D}

\section{O formalismo de Newman-Penrose}

Uma forma alternativa de apresentar a relatividade geral é o formalismo das tetradas. Ao invés de expressar grandezas com vetores e tensores, quantidades relevantes, em termos de uma base das coordenadas, $\partial_{\mu}=\partial / \partial x^{\mu}$, escolhe-se uma base tetrada, que corresponde a quatro vetores linearmente independentes, e projeta-se tais quantidades nessa base. $\mathrm{O}$ formalismo de Newman-Penrose é um formalismo das tetradas com uma escolha para os vetores da base. Neste, os vetores são nulos, ou seja, a base é nula. No trabalho An approach to gravitational radiation by a method of spin coefficients [39], publicado em 1962, Ezra Newman e Roger Penrose utilizaram as tetradas para estudar a radiação gravitacional. Mas tal formalismo, como será visto, também é útil para o caso da radiação eletromagnética, pois ambas percorrem geodésicas do tipo nula. Para realizar tal trabalho, os autores obtiveram 12 constantes, chamadas coeficientes de spin, que foram importantes para o cálculo de quantidades óticas nesta tese. Este formalismo mostra-se útil por simplificar as equações envolvidas, todas somente de primeira ordem, não envolvendo, como já foi dito, uma base das coordenadas quando um tensor é escrito. Usando a seguinte tetrada nula ${ }^{1}\left\{e_{a \mu}\right\}=\left(m_{\mu}, \bar{m}_{\mu}, l_{\mu}, k_{\mu}\right)$ ou, como será conveniente a seguir, $\left\{e_{a}\right\}=(m, \bar{m}, l, k)$, onde a barra significa complexo conjugado, temos, por definição,

$$
k^{\mu} k_{\mu}=l^{\mu} l_{\mu}=m^{\mu} m_{\mu}=\bar{m}^{\mu} \bar{m}_{\mu}=0
$$

e as seguintes relações de ortogonalidade

$$
k^{\mu} m_{\mu}=k^{\mu} \bar{m}_{\mu}=l^{\mu} m_{\mu}=l^{\mu} \bar{m}_{\mu}=0 .
$$

\footnotetext{
${ }^{1}$ Para a tetrada em questão, o índice grego $\mu$ é o tensorial, vai de 0 a 3 . O índice latino é o que identifica os componentes da tetrada, vai de 1 a $4\left(e_{1 \mu}=m_{\mu}, e_{2 \mu}=\bar{m}_{\mu}, e_{3 \mu}=l_{\mu}, e_{4 \mu}=k_{\mu}\right)$.
} 
Além das relações citadas acima, temos

$$
k^{\mu} l_{\mu}=-m^{\mu} \bar{m}_{\mu}=-1, \quad m^{\mu}=\frac{\sqrt{2}}{2}\left(a^{\mu}+i b^{\mu}\right), \quad a^{\mu} a_{\mu}=b^{\mu} b_{\mu}=-\frac{1}{2} .
$$

Na base das coordenadas, $\partial_{\mu}$, podemos expressar a tetrada como

$$
e_{a}=e_{a}^{\mu} \frac{\partial}{\partial x^{\mu}}
$$

Sua correspondente dual, $e^{a} \equiv \omega^{a}$, igualmente na base das coordenadas, escreve-se na seguinte forma:

$$
\omega^{a}=\omega_{\mu}^{a} d x^{\mu}
$$

Para subir e descer índices tensoriais, usamos a métrica e sua inversa. Assim

$$
e_{a}^{\mu}=g^{\mu v} e_{a v}
$$

Podemos também usar a inversa de $e_{a}^{\mu}$, isto é, $\omega_{\mu}^{a}$, para que as relações entre vetores, no espaço tangente, e covetores ou 1-formas, no espaço dual, com suas respectivas componentes, sejam satisfeitas:

$$
e_{a}^{\mu} \omega_{\mu}^{b}=\delta_{a}^{b} \quad \text { e } \quad e_{a}^{\mu} \omega_{v}^{a}=\delta_{v}^{\mu}
$$

Por fim, assumimos que

$$
e_{a}^{\mu} e_{b \mu}=g^{\mu v} e_{a v} e_{b \mu}=\eta_{a b}
$$

Para subir e descer índices da tetrada $\left(e_{a \mu}=\eta_{a b} e_{\mu}^{b}=\eta_{a b} \omega_{\mu}^{b}\right)$, usa-se a métrica plana $\eta_{a b}$, definida acima, que pode ser expressa na forma matricial - quando a tetrada escolhida é a de Newman-Penrose - como:

$$
\eta_{a b}=\eta^{a b}=\left(\begin{array}{cccc}
0 & 1 & 0 & 0 \\
1 & 0 & 0 & 0 \\
0 & 0 & 0 & -1 \\
0 & 0 & -1 & 0
\end{array}\right)
$$

Das definições e construções acima, segue que o tensor métrico pode ser escrito em termos da base nula:

$$
\begin{aligned}
g_{\mu \nu} & =e_{a \mu} e_{b \nu} \eta^{a b} \\
& =m_{\mu} \bar{m}_{\nu}+\bar{m}_{\mu} m_{\nu}-l_{\mu} k_{\nu}-k_{\mu} l_{\nu}
\end{aligned}
$$


Transformações de Lorentz, aplicadas à tetrada nula, foram importantes na abordagem realizada por Pineault e Roeder [37] para o estudo de propriedades óticas geométricas da métrica de Kerr. Como seguimos esse mesmo caminho, essa mesma abordagem, para as métricas construídas no capítulo 4 , convém listar transformações nulas para a tetrada $\left\{e_{a}\right\}=$ $(m, \bar{m}, l, k)$, pois foram utilizadas pelos autores citados:

\section{Rotações nulas ( $l$ fixo)}

$$
l^{\prime \mu}=l^{\mu}, m^{\prime \mu}=m^{\mu}+E l^{\mu}, k^{\prime \mu}=k^{\mu}+E \bar{m}^{\mu}+\bar{E} m^{\mu}+E \bar{E} l^{\mu}, \text { com } E \text { complexo. }
$$

\section{Rotações nulas ( $k$ fixo)}

$$
k^{\prime \mu}=k^{\mu}, m^{\prime \mu}=m^{\mu}+B k^{\mu}, l^{\prime \mu}=l^{\mu}+B \bar{m}^{\mu}+\bar{B} m^{\mu}+B \bar{B} l^{\mu}, \text { com } B \text { complexo. }
$$

\section{Rotações espaciais no plano $m-\bar{m}$}

$$
m^{\prime \mu}=e^{-i \chi} m^{\mu}, \text { com } \chi \text { real. }
$$

\section{Transformações especiais de Lorentz (boosts) no plano $k-l$}

$$
k^{\prime \mu}=A k^{\mu}, \quad l^{\prime \mu}=A^{-1} l^{\mu}, \quad \operatorname{com} A>0 .
$$

Vale a pena ressaltar que os quatro tipos de transformações acima, por meio de $E, B, \chi$ e $A$, possuem no total seis parâmetros reais. Por fim, temos:

\section{Transformações mais gerais que preservam a direção $k$}

$$
k^{\prime \mu}=A k^{\mu}, \quad m^{\prime \mu}=e^{-i \chi}\left(m^{\mu}+B k^{\mu}\right), \quad l^{\prime \mu}=A^{-1}\left(l^{\mu}+B \bar{m}^{\mu}+\bar{B} m^{\mu}+B \bar{B} k^{\mu}\right) .
$$

Vamos obter, a partir de agora, os chamados coeficientes de spin, que são escalares importantes na caracterização de espaços-tempo. Para tal feito, utilizamos a equação da derivada covariante direcional para a base $\left\{e_{a \mu}\right\}$ com o intuito de construir os chamados coeficientes de rotação de Ricci $^{2}$. Utilizando o operador mencionado na base tetrada, obtivemos

$$
\begin{aligned}
e_{b}{ }^{v} \nabla_{v} e_{a \mu} & =e_{b}^{v} \partial_{v} e_{a \mu}+\Gamma_{v \mu}^{\gamma} e_{a \gamma} e_{b}^{v} \\
& =\Gamma_{\alpha \mu \nu} g^{\alpha \gamma} e_{a \gamma} e_{b}{ }^{v} \\
& =\Gamma_{\alpha \mu \nu} e_{a}^{\alpha} e_{b}{ }^{v}
\end{aligned}
$$

\footnotetext{
${ }^{2}$ Os coeficientes de spin, como veremos, são combinações lineares dos coeficientes de rotação de Ricci.
} 
onde usamos o fato de que a derivada parcial de $e_{a}$, na direção $e_{b}$, é nula e a simetria $\Gamma_{\mu \nu}^{\gamma}=\Gamma_{v \mu}^{\gamma}$. Quando multiplicamos ambos os lados de (D.16) por $e_{c}^{\mu}$, chegamos ao seguinte resultado:

$$
e_{c}^{\mu} e_{b}^{v} \nabla_{v} e_{a \mu}=\Gamma_{\alpha \mu \nu} e_{a}^{\alpha} e_{c}^{\mu} e_{b}^{v}=\Gamma_{a b c} .
$$

As componentes do tensor $\Gamma_{\alpha \mu \nu}$, escritas na base tetrada $\left(\Gamma_{a b c}\right)$, são conhecidas como coeficientes de rotação de Ricci. Podemos escrevê-los como

$$
\Gamma_{c a b}=e_{c}^{\mu} \nabla_{v} e_{a \mu} e_{b}^{v}
$$

Os coeficientes de rotação de Ricci são antissimétricos nos primeiros dois índices: $\Gamma_{c a b}=-\Gamma_{a c b}$. Escritos em termos da tetrada tetrada nula, conduzem aos chamados coeficientes de spin, que nada mais são do que combinações lineares dos coeficientes de rotação. As doze combinações lineares, os doze coeficientes de spin, são:

$$
\begin{aligned}
\kappa & \equiv-\Gamma_{144}=-\nabla_{v} k_{\mu} m^{\mu} k^{v}, \\
\rho & \equiv-\Gamma_{142}=-\nabla_{v} k_{\mu} m^{\mu} \bar{m}^{v}, \\
\sigma & \equiv-\Gamma_{141}=-\nabla_{v} k_{\mu} m^{\mu} m^{v}, \\
\tau & \equiv-\Gamma_{143}=-\nabla_{v} k_{\mu} m^{\mu} l^{v}, \\
v & \equiv \Gamma_{233}=\nabla_{v} l_{\mu} \bar{m}^{\mu} l^{v}, \\
\mu & \equiv \Gamma_{231}=\nabla_{v} l_{\mu} \bar{m}^{\mu} m^{v}, \\
\lambda & \equiv \Gamma_{232}=\nabla_{v} l_{\mu} \bar{m}^{\mu} \bar{m}^{v}, \\
\pi & \equiv \Gamma_{234}=\nabla_{v} l_{\mu} \bar{m}^{\mu} k^{v}, \\
\varepsilon & \equiv \frac{1}{2}\left(\Gamma_{214}-\Gamma_{344}\right)=\frac{1}{2}\left(\nabla_{v} m_{\mu} \bar{m}^{\mu} k^{v}-\nabla_{v} k_{\mu} l^{\mu} k^{v}\right), \\
\beta & \equiv \frac{1}{2}\left(\Gamma_{211}-\Gamma_{341}\right)=\frac{1}{2}\left(\nabla_{v} m_{\mu} \bar{m}^{\mu} m^{v}-\nabla_{v} k_{\mu} l^{\mu} m^{v}\right), \\
\gamma & \equiv \frac{1}{2}\left(\Gamma_{433}-\Gamma_{123}\right)=\frac{1}{2}\left(\nabla_{v} l_{\mu} k^{\mu} l^{v}-\nabla_{v} \bar{m}_{\mu} m^{\mu} l^{v}\right), \\
\alpha & \equiv \frac{1}{2}\left(\Gamma_{432}-\Gamma_{122}\right)=\frac{1}{2}\left(\nabla_{v} l_{\mu} k^{\mu} \bar{m}^{v}-\nabla_{v} \bar{m}_{\mu} m^{\mu} \bar{m}^{v}\right) .
\end{aligned}
$$

Com os coeficientes de spin, podemos construir o tensor de curvatura ${ }^{3}$ em termos da tetrada nula e obter as chamadas equações de Newman-Penrose ou identidades de Ricci, que medem a variação dos coeficientes listados acima numa direção qualquer da tetrada ${ }^{4}(m, \bar{m}, l, k)$.

\footnotetext{
${ }^{3} \mathrm{O}$ tensor de curvatura, $R_{b c d}^{a}$, é importante pois suas características denotam características do espaço-tempo.

${ }^{4}$ Cf. [39] e, também com um pouco mais de detalhes, [68]. Existe uma diferença no sinal dos coeficientes de spin entre as referências [39] e [68] que se justifica pela não igualdade entre as assinaturas das métricas usadas. Seguimos o caminho exposto em [68].
} 
Uma maneira eficiente para o cálculo das componentes do tensor de curvatura é o procedimento desenvolvido por Cartan. Nesse caminho, defini-se a 2-forma da curvatura

$$
\Theta_{b}^{a} \equiv \frac{1}{2} R_{b c d}^{a} \omega^{c} \wedge \omega^{d}
$$

onde o símbolo $\wedge$ significa o produto exterior (produto entre 1-formas), que conduz à segunda equação de $\operatorname{Cartan}^{5}$

$$
d \Gamma_{b}^{a}+\Gamma_{c}^{a} \wedge \Gamma_{b}^{c}=\Theta_{b}^{a} .
$$

O operador $d$ é a derivada exterior, aplicada a 1-forma da conexão, $\Gamma_{b}^{a} \equiv \Gamma_{b c}^{a} \omega^{c}$. A equação acima é equivalente à definição do tensor de curvatura. Para a tetrada considerada, $(m, \bar{m}, l, k)$, a segunda equação de Cartan resume-se a apenas três equações

$$
\begin{aligned}
d \Gamma_{41}+\Gamma_{41} \wedge\left(\Gamma_{21}+\Gamma_{43}\right) & =\frac{1}{2} R_{41 c d} \omega^{c} \wedge \omega^{d}, \\
d \Gamma_{32}-\Gamma_{32} \wedge\left(\Gamma_{21}+\Gamma_{43}\right) & =\frac{1}{2} R_{32 c d} \omega^{c} \wedge \omega^{d}, \\
d\left(\Gamma_{21}+\Gamma_{43}\right)+2 \Gamma_{32} \wedge \Gamma_{41} & =\frac{1}{2}\left(R_{21 c d}+R_{43 c d}\right) \omega^{c} \wedge \omega^{d},
\end{aligned}
$$

onde os índices numéricos referem-se a tetrada. A 1-forma da conexão pode se escrita como

$$
\Gamma_{a b}=\Gamma_{a b c} \omega^{c}
$$

Com o uso de (D.17), essa mesma 1-forma fica

$$
\Gamma_{a b}=\Gamma_{\alpha \mu v} e_{a}^{\alpha} e_{c}^{\mu} e_{b}^{v} \omega^{c}
$$

Assim, pode-se notar que $\Gamma_{41}=\bar{\Gamma}_{42}$ ou $\Gamma_{21}=\bar{\Gamma}_{12}$, por exemplo. Pois a troca do índice $1 \mathrm{da}$ tetrada pelo índice 2 ou vice-versa indica a troca de $m$ por $\bar{m}$ ou vice-versa.

Para obter as equações de Newman-Penrose, é necessário escrever (D.22) usando os coeficientes de spin (D.19) listados acima. Mas para isso, escrevemos as componentes da conexão na base dual em termos dos mesmos,

$$
\begin{aligned}
\Gamma_{14} & =\Gamma_{14 c} \omega^{c}=-\sigma \omega^{1}-\rho \omega^{2}-\tau \omega^{3}-\kappa \omega^{4}, \\
\Gamma_{23} & =\Gamma_{23 c} \omega^{c}=\mu \omega^{1}+\lambda \omega^{2}+v \omega^{3}+\pi \omega^{4}, \\
\frac{1}{2}\left(\Gamma_{12}+\Gamma_{34}\right) & =-\beta \omega^{1}-\alpha \omega^{2}-\gamma \omega^{3}-\varepsilon \omega^{4},
\end{aligned}
$$

\footnotetext{
${ }^{5}$ Já que usamos a segunda equação de Cartan em nosso texto, vale a pena citar a primeira: $d \omega^{a}=-\Gamma_{b}^{a} \wedge \omega^{b}$, onde $\Gamma_{b}^{a} \equiv \Gamma_{b c}^{a} \omega^{c}$ é a 1-forma da conexão.
} 
e definimos as derivadas direcionais

$$
\begin{aligned}
D \equiv k^{\mu} \nabla_{\mu}, & \Delta \equiv l^{\mu} \nabla_{\mu}, \\
\delta \equiv m^{\mu} \nabla_{\mu}, & \bar{\delta} \equiv \bar{m}^{\mu} \nabla_{\mu},
\end{aligned}
$$

que serão usadas no lado esquerdo das componentes da segunda equação de Cartan escritas na base nula (D.22). As derivadas direcionais medem variações nas direções $k, l, m$ e $\bar{m} \mathrm{e}$ indicam-nos como os coeficientes de spin variam em cada uma direção dos vetores da base. Agora, no lado direito da equação (D.22), utilizamos a decomposição do tensor de Riemann ou de curvatura

$$
R_{\mu v \gamma \delta}=C_{\mu v \gamma \delta}+E_{\mu v \gamma \delta}+G_{\mu v \gamma \delta},
$$

que define em 4 dimensões o tensor de Weyl $C_{\mu v \gamma \delta}$, sendo

$$
\begin{aligned}
E_{\mu v \gamma \delta} & \equiv \frac{1}{2}\left(g_{\mu \gamma} S_{v \delta}+g_{v \delta} S_{\mu \gamma}-g_{\mu \delta} S_{v \gamma}-g_{v \gamma} S_{\mu \delta}\right), \\
G_{\mu v \gamma \delta} & \equiv \frac{1}{12} R\left(g_{\mu \gamma \delta} g_{v \delta}-g_{\mu \delta} g_{v \gamma}\right) \equiv \frac{1}{12} R g_{\mu v \gamma \delta}, \\
S_{\mu v} & \equiv R_{\mu v}-\frac{1}{4} R g_{\mu v},
\end{aligned}
$$

onde $R=R_{\mu}^{\mu}$ é o escalar de Ricci, conforme a notação de [68]. Definem-se, em termos da tetrada nula, os escalares

$$
\begin{aligned}
\Phi_{00} & \equiv \frac{1}{2} S_{\mu \nu} k^{\mu} k^{v}=\frac{1}{2} R_{44} \\
\Phi_{01} & \equiv \frac{1}{2} S_{\mu v} k^{\mu} m^{v}=\frac{1}{2} R_{41} \\
\Phi_{02} & \equiv \frac{1}{2} S_{\mu \nu} m^{\mu} m^{v}=\frac{1}{2} R_{11} \\
\Phi_{11} & \equiv \frac{1}{4} S_{\mu \nu}\left(k^{\mu} l^{v}+m^{\mu} \bar{m}^{v}\right)=\frac{1}{4}\left(R_{43}+R_{12}\right), \\
\Phi_{12} & \equiv \frac{1}{2} S_{\mu \nu} l^{\mu} k^{v}=\frac{1}{2} R_{31}, \\
\Phi_{22} & \equiv \frac{1}{2} S_{\mu \nu} l^{\mu} l^{v}=\frac{1}{2} R_{33},
\end{aligned}
$$

e os coeficientes ou escalares complexos que representam as 10 componentes independentes do tensor de Weyl: 


$$
\begin{aligned}
& \Psi_{0} \equiv C_{\mu v \gamma \delta} k^{\mu} m^{\mu} k^{\gamma} m^{\delta}, \\
& \Psi_{1} \equiv C_{\mu v \gamma \delta} k^{\mu} l^{\mu} k^{\gamma} m^{\delta}, \\
& \Psi_{2} \equiv-C_{\mu v \gamma \delta} k^{\mu} m^{\mu} l^{\gamma} \bar{m}^{\delta}, \\
& \Psi_{3} \equiv C_{\mu v \gamma \delta} l^{\mu} k^{\mu} l^{\gamma} \bar{m}^{\delta}, \\
& \Psi_{4} \equiv C_{\mu v \gamma \delta}{ }^{\mu} \bar{m}^{\mu} l^{\gamma} \bar{m}^{\delta} .
\end{aligned}
$$

Os cinco $\Psi^{\prime} s$ definidos acima, segundo a interpretação de Szekeres [69], têm os seguintes significados físicos: $\Psi_{4}$ representa uma onda transversal na direção $k$; $\Psi_{3}$, uma componente de onda longitudinal; $\Psi_{2}$, um termo de "Coulomb"; $\Psi_{0}$ e $\Psi_{1}$ representam uma componente de uma onda transversal e uma componente de uma onda longitudinal, respectivamente, na direção $l$. Suas leis de transformação, de acordo com (D.11) e (D.12), são:

$$
\begin{aligned}
l^{\mu} \text { fixo: } \Psi_{4}^{\prime} & =\Psi_{4}, \quad \Psi_{3}^{\prime}=\Psi_{3}+E \Psi_{4}, \\
\Psi_{2}^{\prime} & =\Psi_{2}+2 E \Psi_{3}+E^{2} \Psi_{4}, \\
\Psi_{1}^{\prime} & =\Psi_{1}+3 E \Psi_{2}+3 E^{2} \Psi_{3}+E^{3} \Psi_{4} \\
\Psi_{0}^{\prime} & =\Psi_{0}+4 E \Psi_{1}+6 E^{2} \Psi_{2}+4 E^{3} \Psi_{3}+E^{4} \Psi_{4} . \\
k^{\mu} \text { fixo: } \Psi_{0}^{\prime} & =\Psi_{0}, \quad \Psi_{1}^{\prime}=\Psi_{1}+\bar{B} \Psi_{0}, \\
\Psi_{2}^{\prime} & =\Psi_{2}+2 \bar{B} \Psi_{1}+\bar{B}^{2} \Psi_{0}, \\
\Psi_{3}^{\prime} & =\Psi_{3}+3 \bar{B} \Psi_{2}+3 \bar{B}^{2} \Psi_{1}+\bar{B}^{3} \Psi_{0}, \\
\Psi_{4}^{\prime} & =\Psi_{4}+4 \bar{B} \Psi_{3}+6 \bar{B}^{2} \Psi_{2}+4 \bar{B}^{3} \Psi_{1}+\bar{B}^{4} \Psi_{0} .
\end{aligned}
$$

Com as definições dos $\Phi^{\prime} s$ e dos $\Psi^{\prime} s$ acima, a segunda equação de Cartan, escrita na tetrada nula $\{m, \bar{m}, l, k\}$, transforma-se, então, nas equações de Newman-Penrose:

$$
\begin{aligned}
D \rho-\bar{\delta} \kappa & =\rho^{2}+\sigma \bar{\sigma}+(\varepsilon+\bar{\varepsilon}) \rho-\bar{\kappa} \tau-(3 \alpha+\bar{\beta}-\pi) \kappa+\Phi_{00} \\
D \sigma-\delta \kappa & =(\rho+\bar{\rho}) \sigma+(3 \varepsilon-\bar{\varepsilon}) \sigma-(\tau-\bar{\pi}+\bar{\alpha}+3 \beta) \kappa+\Psi_{0} \\
D \tau-\Delta \kappa & =(\tau+\bar{\pi}) \rho+(\bar{\tau}+\pi) \sigma+(\varepsilon-\bar{\varepsilon}) \tau-(3 \gamma+\bar{\gamma}) \kappa+\Psi_{1}+\Phi_{01} \\
D \alpha-\bar{\delta} \varepsilon & =(\rho+\bar{\varepsilon}-2 \varepsilon) \alpha+\beta \bar{\sigma}-\bar{\beta} \varepsilon-\kappa \lambda-\bar{\kappa} \gamma+(\varepsilon+\rho) \pi+\Phi_{10} \\
D \beta-\delta \varepsilon & =(\alpha+\pi) \sigma+(\bar{\rho}-\varepsilon) \beta-(\mu+\gamma) \kappa-(\bar{\alpha}-\bar{\pi}) \varepsilon+\Psi_{1} \\
D \gamma-\Delta \varepsilon & =(\tau+\bar{\pi}) \alpha+(\bar{\tau}+\pi) \beta-(\varepsilon+\bar{\varepsilon}) \gamma-(\gamma+\bar{\gamma}) \varepsilon+\tau \pi-v \kappa+\Psi_{2} \\
& +\Phi_{11}-\frac{R}{24}
\end{aligned}
$$




$$
\begin{aligned}
D \lambda-\bar{\delta} \pi & =\rho \lambda+\bar{\sigma} \mu+\pi^{2}+(\alpha-\bar{\beta}) \pi-v \bar{\kappa}-(3 \varepsilon-\bar{\varepsilon}) \lambda+\Phi_{20} \\
D \mu-\delta \pi & =\bar{\rho} \mu+\sigma \lambda+\pi \bar{\pi}-(\varepsilon+\bar{\varepsilon}) \mu-\pi(\bar{\alpha}-\beta)-v \kappa+\Psi_{2}+\frac{R}{12} \\
D v-\Delta \pi & =(\pi+\bar{\tau}) \mu+(\bar{\pi}+\tau) \lambda+(\gamma-\bar{\gamma}) \pi-(3 \varepsilon+\bar{\varepsilon}) v+\Psi_{3}+\Phi_{21} \\
\Delta \lambda-\bar{\delta} v & =-(\mu+\bar{\mu}) \lambda-(3 \gamma-\bar{\gamma}) \lambda+(3 \alpha+\bar{\beta}+\pi-\bar{\tau}) v-\Psi_{4} \\
\delta \rho-\bar{\delta} \sigma & =(\bar{\alpha}+\beta) \rho-(3 \alpha-\bar{\beta}) \sigma+(\rho-\bar{\rho}) \tau+(\mu-\bar{\mu}) \kappa-\Psi_{1}+\Phi_{01} \\
\delta \alpha-\bar{\delta} \beta & =\mu \rho-\lambda \sigma+\alpha \bar{\alpha}+\beta \bar{\beta}-2 \alpha \beta+(\rho-\bar{\rho}) \gamma+(\mu-\bar{\mu}) \varepsilon \\
\delta \lambda-\bar{\delta} \mu & =(\rho-\bar{\rho}) v+(\mu-\bar{\mu}) \pi+(\alpha+\bar{\beta}) \mu+(\bar{\alpha}+3 \beta) \lambda-\Psi_{3}+\Phi_{21} \\
\delta v-\Delta \mu & =\mu \Phi_{2}+\lambda \bar{\lambda}+(\gamma+\bar{\gamma}) \mu-\bar{v} \pi+(\tau-3 \beta-\bar{\alpha}) v+\Phi_{22} \\
\delta \gamma-\Delta \beta & =(\tau-\bar{\alpha}-\beta) \gamma+\mu \tau-\sigma v-\varepsilon \bar{v}-(\gamma-\bar{\gamma}-\mu) \beta+\alpha \bar{\lambda}+\Phi_{12} \\
\delta \tau-\Delta \sigma & =\mu \sigma+\bar{\lambda} \rho+(\tau+\beta-\bar{\alpha}) \tau-(3 \gamma-\bar{\gamma}) \sigma-\kappa \bar{v}+\Phi_{02} \\
\Delta \rho-\bar{\delta} \tau & =-(\rho \bar{\mu}+\sigma \lambda)+(\bar{\beta}-\alpha-\bar{\tau}) \tau+(\gamma+\bar{\gamma}) \rho+v \kappa-\Psi_{2}-\frac{R}{12} \\
\Delta \alpha-\bar{\delta} \gamma & =(\rho+\varepsilon) v-(\tau+\beta) \lambda+(\bar{\gamma}-\mu) \alpha+(\bar{\beta}-\bar{\tau}) \gamma-\Psi_{3}
\end{aligned}
$$




\section{Apêndice E}

\section{Os referenciais localmente não girantes}

Em 1972, Bardeen, Press e Teukolsky publicaram o trabalho Rotating black holes: locally nonrotating frames, energy extration, and scalar synchrotron radiation [70], no qual o formalismo dos referenciais localmente não girantes - tetradas ortonormais que rodam com a geometria - foi adotado para o estudo de fenômenos no entorno do buraco negro de Kerr. Segundo os autores: "A Física não é simples nas coordenadas de Boyer-Lindquist, pois (i) o arrasto dos referenciais inercias torna-se tão forte que o vetor $\partial / \partial t$ da base das coordenadas tende a um vetor do tipo espaço quando próximo do limite estático $r_{0}$ ", que é a fronteira da ergosfera, e "(ii) a métrica não é diagonal, então subir e descer índices tensoriais tipicamente introduz complexidade algébrica."1 Para superar essas dificuldades, consideraram a métrica geral com simetria axial (B.11), escrita nas coordenadas $(t, r, \theta, \varphi)$,

$$
d s^{2}=-e^{2 v} d t^{2}+e^{2 \psi}(d \varphi-\Omega d t)^{2}+e^{2 \lambda} d r^{2}+e^{2 \mu} d \theta^{2}
$$

Escolheram, então, um referencial localmente não girante, onde: $r=$ const., $\theta=$ const., $\varphi=\Omega t+$ const ; assim, a tetrada ortonormal $\left\{e_{(a)}\right\}=\left\{e_{(t)}, e_{(r)}, e_{(\theta)}, e_{(\varphi)}\right\}$ que gira com o buraco negro pôde ser escrita em termos de uma base das coordenadas, $\partial_{\mu}$, de acordo com a definição (D.4), como

$$
\begin{aligned}
& e_{(1)}=e_{(t)}=e^{-v}\left(\frac{\partial}{\partial t}+\Omega \frac{\partial}{\partial \varphi}\right) \\
& e_{(2)}=e_{(r)}=e^{-\lambda} \frac{\partial}{\partial r} \\
& e_{(3)}=e_{(\theta)}=e^{-\mu} \frac{\partial}{\partial \theta} \\
& e_{(4)}=e_{(\varphi)}=e^{-\psi} \frac{\partial}{\partial \varphi} .
\end{aligned}
$$

${ }^{1}$ Cf. [70], p. 353. 
Sua base dual correspondente $\left\{e^{(a)}\right\}$, de acordo com (D.5), fica:

$$
\begin{aligned}
e^{(1)} & =e^{(t)}=e^{v} d t, \\
e^{(2)} & =e^{(r)}=e^{\lambda} d r, \\
e^{(3)} & =e^{(\theta)}=e^{\mu} d \theta \\
e^{(4)} & =e^{(\varphi)}=e^{\psi}(d \varphi-\Omega d t) .
\end{aligned}
$$

Os índices entre parênteses na tetrada indicam o referencial não girante. Nota-se, por (E.2) e (E.3), que os vetores da tetrada satisfazem:

$$
e_{(a)}^{\mu} e_{(b) \mu}= \begin{cases}0, & \text { se } a \neq b \\ -1, & \text { se } a=b=t \\ 1, & \text { se } a=b \text { e } a=r, \text { ou } a=\theta, \text { ou } a=\varphi\end{cases}
$$

Ou seja, a tetrada ortonormal é composta por um vetor tipo tempo e três do tipo espaço. A constante $\Omega$, que foi interpretada no artigo dos autores citados como velocidade angular do buraco negro, no caso da métrica de Kerr, como pode ser vista no capítulo 12 de [49], é definida pela expressão

$$
\Omega=-\frac{g_{t \varphi}}{g_{\varphi \varphi}} .
$$

A relação entre um tensor qualquer na base não girante, com índices escritos entre parênteses, e um tensor escrito na base das coordenadas é dada por

$$
T_{(a)(b)}=e_{(a)}^{\mu} e_{(b)}^{v} T_{\mu v} \quad \text { e } \quad T_{\mu \nu}=e_{\mu}^{(a)} e_{v}^{(b)} T_{(a)(b)}
$$

sendo $e_{(a)}^{\mu}$ e $e_{\mu}^{(a)}$ matrizes que podem ser escritas, conforme (E.2) e (E.3), como

$$
e_{(a)}^{\mu}=\left(\begin{array}{cccc}
e^{-v} & 0 & 0 & 0 \\
0 & e^{-\lambda} & 0 & 0 \\
0 & 0 & e^{-\mu} & 0 \\
\Omega e^{-v} & 0 & 0 & e^{-\psi}
\end{array}\right)
$$

$\mathrm{e}$

$$
e_{\mu}^{(a)}=\left(\begin{array}{cccc}
e^{v} & 0 & 0 & 0 \\
0 & e^{\lambda} & 0 & 0 \\
0 & 0 & e^{\mu} & 0 \\
-\Omega e^{\psi} & 0 & 0 & e^{\psi}
\end{array}\right)
$$


O formalismo dos referencias localmente não girantes foi usado por nós, no capítulo 5, com o auxílio da abordagem de Pineault e Roeder, para realizar um estudo de ótica geométrica das métricas com simetria axial num contexto de mundos brana expostas no capítulo 4 . 



\section{Referências Bibliográficas}

[1] SCHWARZSCHILD, K. Über das gravitationsfeld eines massenpunktes nach der einsteinschen theorie. Sitzungsberichte der Königlich-Preussischen Akademie der Wissenschaften. 189, 1916.

[2] REISSNER, H. Über die eigengravitation des elektrischen felds nach der einsteinschen theorie. Annalen der Physik, v. 50, 106, 1916.

[3] NORDSTRÖM, G. On the energy of the gravitational field in Einstein's theory. Proc. Kon. Ned. Akad. Wet., v. 20, 1238, 1918.

[4] KERR, R. P. Gravitational field of a spinning mass as an example of algebraically special metrics. Physical Review Letters, v. 11, 26, 1963.

[5] NEWMAN, E. T.; COUCH, E.; CHINNAPARED, K.; EXTON, A.; PRAKASH, A.; TORRENCE, R. Metric of rotating charged mass. Jounal of Mathematical Physics, v. 3, 566, 1965.

[6] CARTER, B. Hamilton-Jacobi and Schrodinger separable solutions of Einstein's equations. Communications in Mathemathical Physics, v. 10, 280, 1968.

[7] MAYERS, R. C.; PERRY, M. J. Black holes in higher dimensional spacetimes. Annals of Physics, v. 172, 304, 1986.

[8] GIBBONS, G. W.; LÜ H.; PAGE, D. N.; POPE, C. N. The general Kerr-de Sitter metrics in all dimensions. Journal of Geometry and Physics, v. 53, 49, 2005.

[9] HAWKING, S. W.; HUNTER, C. J.; TAYLOR-ROBINSON, M. M. Rotation and the AdS/CFT correspondence. Physical Review D, v. 59, 064005, 1999.

[10] MORRIS, M. S.; THORNE, K. S. Wormholes in spacetime and their use for interstellar travel: A tool for teaching general relativity. American Journal of Physics, v. 56, 395, 1988. 
[11] RIESS, A. G. et al. (Supernova Search Team Collaboration). Observational evidence from supernovae for an accelerating universe and a cosmological constant. The Astronomical Journal, v. 116, 1009, 1998.

[12] PERLMUTTER, S. et al. (Supernova Cosmology Project Collaboration). Measurements of $\Omega$ and $\Lambda$ from 42 high-redshift supernovae. The Astrophysical Journal, v. 517, 565, 1999.

[13] KORMENDY, J.; RICHSTONE, D. The Search for Supermassive black holes in galatic nuclei. Annual Review of Astronomy and Astrophysics v. 33, 581, 1995.

[14] HAWKING, S.W.; ELLIS, G. F. R. The Large Scale Structure of Space-Time. Cambridge: Cambridge University Press, 1973.

[15] HORAVA, P.; WITTEN, E. Heterotic and type I string dynamics from eleven dimensions. Nuclear Physics B, v. 460, 506, 1996.

[16] LUKAS, A.; OVRUT, B. A. STELLE, K.S.; WALDRAM, D. Universe as a domain wall. Physical Review D, v. 59, 086001,1999.

[17] RANDALL, L.; SUNDRUM, R. An Alternative to compactification. Physical Review Letters, v. 83, 4690, 1999.

[18] RANDALL, L.; SUNDRUM, R. Large Mass Hierarchy from a Small Extra Dimension. Physical Review Letters, v. 83, 3370, 1999.

[19] ARKANI-HAMED, N.; DIMOPOULOS, S.; DVALI, G. The Hierarchy problem and new dimensions at a millimeter. Physics Letters B, v. 429, 263, 1998.

[20] MAARTENS, R. Brane-world gravity. Living Reviews in Relativity. 7, 7, 2004.

[21] CLIFTON, T.; FERREIRA, P. G.; PADILlA, A.; SKORDIS, C. Modified gravity and cosmology. Physics Reports, v. 513, 1, 2012.

[22] BURDMAN, G. New solutions to the hierarchy problem. Brazilian Journal of Physics, v. 37, 2007.

[23] DVALI, G.; GABADADZE, G.; PORRATI, M. 4D gravity on a brane in 5D Minkowski space. Physics Letters B, v. 485, 208, 2000. 
[24] NOVELlO, M.; BERGLIAFFA, S. E. PEREZ. Bouncing cosmologies. Physics Reports, v. 463, 127, 2008.

[25] CHAMBLIN, A.; HAWKING, S. W.; REALL, H. S. Brane-world black holes. Physical Review D, v. 61, 065007, 2000.

[26] SHIROMIZU, T.; MAEDA, K.; SASAKI, M. The Einstein equations on the 3-brane world. Physical Review D, v. 62, 024012, 2000.

[27] CASADIO, R.; FABBRI, A.; MAZZACURATI, L. New black holes in the brane world? Physical Review D, v. 65, 084040, 2002.

[28] BRONNIKOV, K; DEHREN, H.; MELNIKOV, V. General class of braneworld black holes. Physical Review D, v. 68, 024025, 2003.

[29] MOLINA, C.; NEVES, J. C. S. Black holes and wormholes in AdS branes. Physical Review D, v. 82, 044029, 2010.

[30] MOLINA, C.; NEVES, J. C. S. Wormholes in de Sitter branes. Physical Review D, v. 86, 024015, 2012.

[31] MODGIL, M. S.; PANDA, S.; SENGUPTA, G. Rotating brane world black holes. Modern Physics Letters A, v. 17, 23, 2002.

[32] DADHICH, N.; MAARTENS, R.; PAPADOPOULOS, P.; REZANIA, V. Black holes on the brane. Physical Letters B, v. 487, 1, 2000.

[33] ALIEV, A. N.; GÜMRÜKÇÜOGLU, A. E. Charged rotating black holes on a 3-brane. Physical Review D, v. 71, 104027, 2005.

[34] ALIEV, A. N.; GÜMRÜKÇÜOGLU, A. E. Gravitational field equations on and off a 3-brane world. Classical and Quantum Gravity. v. 21, 5081, 2004.

[35] NEVES, J. C. S.; MOLINA, C. Rotating black holes in a Randall-Sundrum brane with a cosmological constant. arXiv:qr-qc/1211.2848, 2012.

[36] BRODUTCH, A.; DEMARIE, T. F.; TERNO, D. R. Photon polarization and geometric phase in general relativity. Physical Review D, v. 84, 104043, 2011.

[37] PINEAULT, S.; ROEDER, R. C. Applications of geometrical optics to the Kerr metric. The Astrophysical Journal, v. 212, 541, 1977. 
[38] SU, F. S. O.; MALLETT, R. L. The effect of the Kerr metric on the plane of polarization of an electromagnetic wave. The Astrophysical Jounal, v. 238, 1980.

[39] NEWMAN, E.; PENROSE, R. An approach to gravitacional radiation by a method of spin coefficients. Jounal of Mathematical Physics, v. 3, n. 3, 566, 1962.

[40] HOLE, C. D.; SCHMIDT, U.; HECKEL, B. R.; ADELBERGER, E. G.; GUNDLACH, J. H. KAPNER, D. J.; SWANSON, H. E. Sub-millimeter tests of the gravitational inverse-square law: A search for "large" extra dimensions. Physical Review Letters, v. 86, 1418, 2001.

[41] GREGORY, R. Black string instabilities in anti-de Sitter space. Classical and Quantum Gravity. v. 17, L125, 2000.

[42] GREGORY, R.; LAFLAMME, R. Black strings and p-branes are unstable. Physical Review Letters, v. 70, 2837, 1993.

[43] CREEK, S.; GREGORY, R.; KANTI, P.; MISTRY, B. Braneworld stars and black holes. Classical and Quantum Gravity, v. 23, 6633, 2006.

[44] MUKHI, S. String theory: a perspective over the last 25 years. Classical and Quantum Gravity, v. 28, 153001, 2011.

[45] LYKKEN, J. Weak scale superstrings. Physical Review D, v. 54, 3693, 1996.

[46] GREGORY, R.; WHISKER, R.; BECKWITH, K.; DONE, C. Observing braneworld black holes. Journal of cosmology and astroparticle physics, v. 10, 013, 2004.

[47] SEAHRA, S. S. Naked shell singularities on the brane. Physical Review D, v. 71, 084020, 2005.

[48] SEAHRA, S. S.; WESSON, P. Application of the Campbell-Magaard theorem to higher-dimensional physics. Classical and Quantum Gravity. v. 20, 1321, 2003.

[49] WALD, R. M. General Relativity. Chicago: The University of Chicago Press, 1984. 
[50] CARROLL, S. Spacetime and Geometry: an introduction to general relativity. San Francisco: Addison Wesley, 2004.

[51] GIBBONS, G. W.; HAWKING, S. W. Cosmological event horizons, thermodynamics, and particle creation. Physical Review D, v. 15, 2738, 1977.

[52] MAGNON, A. On Komar integrals in asymptotically anti-de Sitter spacetimes. Journal of Mathematics Physics, v. 26, 3112, 1985.

[53] ASHTEKAR, A.; MAGNON, A. Asymptotically anti-de Sitter space-times. Classical and Quantum Gravity. v. 1, L39, 1984.

[54] ASHTEKAR, A; DAS, S. Asymptotically anti-de Sitter space-times: conserved quantities. Classical and Quantum Gravity. v. 17, L17, 2000.

[55] BALASUBRAMANIAN, V.; KRAUSS, P. A stress tensor for anti-de Sitter gravity. Communications in Mathematical Physics. v. 208, 413, 1999.

[56] GHEZELBASH, A. M.; MANN, R B. Action, Mass and Entropy of Schwarzschild-de Sitter black holes and the de Sitter/CFT Correspondence. Journal of High Energy Physics, v. 01, 005, 2002.

[57] BROWN, J.D.; YORK, W. Quasilocal energy and conserved charges from the gravitacional action. Physical Review D, v. 47, 1407, 1993.

[58] CALDARELli, M. M.; COGNOLA G.; KLEMM, D. Thermodynamics of Kerr-Newman-AdS black holes and conformal field theories. Classical and Quantum Gravity, v. 17, 399, 2000.

[59] DEHGHANI, M. H.; KHAJEHAZAD, H. The thermodynamic of a KerrNewman-de Sitter black hole. Canadian Journal of Physics, v. 81, 1363, 2003.

[60] TEITELBOIM, C. Gravitational thermodynamics of Schwarzschild de Sitter space. arXiv:hep-th/0203258v1, 2002.

[61] GIBBONS, G. W.; HAWKING, S. W. Action integrals and partition functions in quantum gravity. Physical Review D, v. 15, 2752, 1977.

[62] SEKIWA, Y. Thermodynamics of de Sitter black holes: Thermal cosmological constant. Physical Review D, v. 73, 084009, 2006.

[63] GOMBEROFF, A.; TEITELBOIM, C. de Sitter black holes with either of the two horizons as a boundary. Physical Review D, v. 67, 104024, 2003. 
[64] CARTER, B. Global structure of the Kerr family of gravitational fields. Physical Review, v. 174, n. 5, 1559, 1968.

[65] CHANDRASEKHAR. S. The mathematical theory of black holes. Oxford: Oxford University Press, 1992.

[66] HACKMANN, E.; LÄMMERZAHL, C.; KAGRAMANOVA, V.; KUNZ, J. Analytical solution of the geodesic equation in Kerr-(anti-) de Sitter spacetimes. Physical Review D, v. 81, 044020, 2010.

[67] D'INVRENO, R. Introducing Einstein's Relativity. 5. ed. Oxford: Oxford University Press, 1992.

[68] STEPHANI, H.; KRAMER, D.; MACCALLUM, M.; HOENSELAERS, C.; HERLT, E. Exact solutions of Einstein's field equations. 2. ed. Cambridge: Cambridge University Press, 2006.

[69] SZEKERES, P. The gravitaional compass. Journal of Mathematical Physics, v. $6,1387$.

[70] BARDEEN, J. M.; PRESS, W. H.; TEUKOLSKY, S. A. Rotating black holes: locally nonrotating frames, energy extration, and scalar synchrotron radiation. The Astrophysical Journal, v. 178, 347, 1972.

[71] MISNER, C. W.; THORNE, K. S.; WHEELER, J. A. Gravitation. New York: W. H. Freeman and Company, 1973.

[72] WALKER, M. Block diagrams and the extension of timelike two-surfaces. Journal of Mathematical Physics, v. 11, 2280, 1970.

[73] VISSER, M. Lorentzian wormholes: from Einstein to Hawking. New York: Springer-Verlag, 1996.

[74] BRONNIKOV, K. A.; ELIZALDE, E.; ODINTSOV, S. D.; ZASLAVSKII, O. B. Horizons versus singularities in spherically symmetric space-times. Physical Review D, v. 78, 064049, 2008.

[75] KERR, R. P.; SCHILD, A. Some algebraically degenerate solutions of Einstein's gravitational field equations. Proceedings of Symposia in Applied Mathematics, v. 17, 199, 1965. 
[76] TAKASHI, T.; MAEDA, H. Spacetime structure of static solutions in GaussBonnet gravity: Neutral case. Physical Review D, v. 71, 124002, 2005.

[77] ALIEV, A. Electromagnetic properties of Kerr-anti-de Sitter black holes. Physical Review D, v. 75, 084041, 2007.

[78] HAWKING, S.W.; PAGE, D.N. Thermodynamic of black holes in anti-de Sitter space. Communications in Mathematical Physics. 87, 577, 1983.

[79] GODFREY, B. B. Mach's Principle, the Kerr Metric, and Black-Hole Physics. Physical Review D, v. 1, 2721, 1970.

[80] HEMMING, S.; THORLACIUS, L. Thermodynamic of large AdS black holes. Journal of High Energy Physics, v. 11, 086, 2007.

[81] BROWN, J.D.; CREIGHTON, J.; MANN, R.B. Temperature, energy, and heat cpacity of asymptotically anti-de Sitter black holes. Physical Review D, v. 50, 6394, 1994.

[82] ISRAEL, W. Singular hypersurfaces and thin shells in general relativity. $\mathrm{Nu}$ ovo Cimento B, v.44, n. 1, 1966.

[83] GIBLIN JR., J. T.; MAROLF, D.; GARVEY, R. Spacetime Embedding Diagrams for Spherically Symetric Black Holes. General Relativity and Gravitation, v. 36, n. 1, 83, 2004. 